\title{
Completeza Assintotica em Teoria Quântica de Campos na Rede
}

\author{
Fernando Auil
}

TESE APRESENTADA

$\mathrm{AO}$

INSTITUTO DE MATEMÁTICA E ESTATÍSTICA

DA

UNIVERSIDADE DE SÃO PAULO

PARA A OBTENÇÃO DO GRAU

$\mathrm{DE}$

DOUTOR EM MATEMÁTICA APLICADA

Área de Concentração: Análise Funcional

Orientador: Prof. Dr. João Carlos Alves Barata

Durante a elaboração deste trabalho, o autor recebeu apoio financeiro do $\mathrm{CNPq}$

-São Paulo, novembro de 2000- 


\section{Completeza Assintótica em Teoria Quântica de Campos na Rede}

Este exemplar corresponde à redação final da tese, devidamente corrigida e defendida por Fernando Auil e aprovada pela comissão julgadora.

São Paulo, novembro de 2000

Banca Examinadora

- Prof. Dr. João Carlos Alves Barata (orientador) - IF-USP

- Prof. Dr. Enrique von Dreifus - IME-USP

- Prof. Dr. Walter Felipe Wreszinski - IF-USP

- Prof. Dr. Eduardo Cantera Marino - IF-UFRJ

- Prof. Dr. Ricardo Schwartz Schor - ICEx-UFMG 


\section{Resumo}

Parafraseando técnicas introduzidas por Spencer e Zirilli em [101], mostra-se de que maneira a completeza assintótica na região de baixas energias em teorias quânticas de campo na rede é basicamente conseqüência da propriedade de decaimento exponencial do núcleo de Bethe-Salpeter e da ausência de estados ligados. Adicionalmente, esta propriedade de decaimento é verificada no caso do modelo de Ising na região de altas temperaturas. Este caso é tratado a título de exemplo da aplicação de técnicas adaptáveis para outros modelos e originariamente introduzidas no contexto da rede por R. Schor, [84], [85], seguindo idéias aplicadas no contínuo por Spencer, [99].

\section{Abstract}

Paraphrasing methods introduced by Spencer and Zirilli in [101], we show how asymptotic completeness in the low energy region for lattice quantum field theories is obtained basically as a consequence of the four particle exponential decay of the Bethe-Salpeter kernel and the absence of bound states. Furthermore, such decay properties are verified for the Ising model at high temperature. This case is considered as an example for the application of methods valid for anothers models and originally introduced in lattice field theories by R. Schor, [84], [85], following ideas used in continous field theories by Spencer, [99]. 


\section{Prefácio}

O presente trabalho aborda a questão da completeza assintótica para teorias quânticas de campo na rede. A abordagem é basicamente uma transcrição ao contexto reticular de resultados obtidos por Spencer e Spencer e Zirilli nos trabalhos assinalados [99] e [101] nas referências, respectivamente.

Na primeira das três partes em que a tese é dividida, são estabelecidas hipóteses gerais sobre a função de 2 pontos e o núcleo de Bethe-Salpeter, sendo mostrada a maneira em como estas se articulam dando lugar à completeza assintótica. Na segunda parte, estas hipóteses são verificadas para um modelo em particular, a saber, o modelo de Ising na região de altas temperaturas. A última parte é constituida apenas por comentários sobre o conteúdo das duas primeiras.

Em primeiro termo, incluiu-se uma suscinta introdução que aborda a Teoria Quântica de Campos, passando pela teoria do espalhamento e teorias na rede, até pesquisas recentes na área da completeza assintótica (capítulo 1). O segundo capítulo introduz as definicões e fatos básicos da teoria na rede. A seção 2.3 inclui o tratamento da teoria de espalhamento na rede, basicamente um resumo dos resutados de [4]. Embora os enunciados apresentados sejam rigorosos do ponto de vista formal, estes dois primeiros capítulos são os mais despretensiosos, incluindo-se apenas a título introdutório, já que resulta impossível justificar o papel orgânico desses resultados dentro da teoria, sem colocá-los no contexto mais amplo da Teoria Quântica de Campos no contínuo, no qual se originaram. O capítulo 3 é o coração da primeira parte. Nele é introduzida a equação de Bethe-Salpeter na rede (seção 3.2 ), sendo destacado o rol resolvente de certos núcleos integrais e a sua relação com a medida espectral do operador de energia-momento (seção 3.3). A seção 3.4 estuda as propriedades de regularidade (analiticidade, compacidade) destes núcleos, constituindo um dos aportes no que esta tese possa ter de original. Estas propriedades são usadas na seção 3.5 para estudar o espectro do operador de energia-momento, e na seção 3.6 é introduzida uma hipótese que permite excluir a presença de estados ligados. Embora esta hipótese seja característica específica dos modelos $P(\varphi)_{2}$ considerados por Spencer, não deixa de resultar interessante destacar que, excetuando este último, os resultados obtidos são conseqüência de suposições bastante gerais sobre o modelo. Finalmente, na procura de informação sobre o espectro absolutamente contínuo, foi necessário restringir-se a dimensão $1+1$, o que possibilita obter uma derivada de Radon-Nikodym da medida espectral (seção 3.7).

A segunda parte inicia-se também com uma introdução, estabelecendo a terminologia e notações a serem utlilizadas, com particular ênfase na expansão em 
polímeros na região de altas temperaturas, já que a literatura a respeito parece não carecer de certa falta de uniformidade no tratamento deste tópico (capítulo 4). Os resultados do capítulo 5 também não tem nada de originais, mas são incluidos para sua posterior utilização no capítulo seguinte, onde são estudadas as propriedades de decaimento do núcleo de Bethe-Salpeter para o modelo de Ising no regime das altas temperaturas. Este caso é considerado meramente a título de exemplo da aplicação de técnicas adaptáveis para outros modelos e que foram originariamente introduzidas no contexto da rede por R. Schor, [84], [85], seguindo idéias aplicadas no contínuo por Spencer, [99].

O contéudo da terceira e última parte nos dispensa de maiores comentários. Apenas que o seu primeiro capítulo aborda exclusivamente questões tratadas neste trabalho, entanto que o seguinte explora especulações de caráter mais geral.

Defrontados quase que continuamente com o lacônico e suscinto estilo "para especialistas" dos artigos e publicações de pesquisa, a suposta relativamente maior liberdade narrativa e estilística que comporta a redação de uma tese de doutoramento tem privilegiado no presente caso, espera-se, uma abordagem "didática". Assim, os enunciados dos resultados técnicos são matematicamente rigorosos e detalhados, e a mesma coisa pretende-se das respectivas demonstrações. Considera-se também que a inclusão de uma razoável lista de referências bibliográficas e algumas citações oportunas venham situar-se no meio termo de compromisso entre o caráter autocontido da exposição e as óbvias limitações de espaço e tempo. Comentários heurísticos, como assim também analogias e exemplos provenientes de outras áreas têm sidos acrescentados, sempre que possível. Tem sido concedido especial ênfase, principalmente na primeira parte, na maneira em como os resultados articulam-se estruturalmente para dar lugar aos resultados esperados, destacando, em geral, o todo, tentando não abrir mão do rigor no tratamento das partes.

No corpo do texto, e para futura referência, cada resultado é enumerado pela sua ordem de aparição junto com o número do capítulo e a seção na qual é enunciado, v.g., 1.2 .3 refere-se ao terceiro resultado na seção 2 do capítulo 1. Idêntico sistema é usado para enumerar as fórmulas e equações, mas neste caso o número aparece entre parênteses, v.g., (1.2.3).

Os principais conceitos definidos são destacados com caracteres em negrito. Num índice remissivo, no final, estão referidos os principais, senão quase a totalidade, dos símbolos e definições empregadas. Algumas notações e convenções constam logo no começo, após o sumário, pois, embora usuais, o seu uso freqüente na literatura aparentemente não consagrou unanimidade a respeito do seu tratamento.

Para evitar a proliferação de notas de rodapé um tanto prolixas, todas as referências bibliográficas constam no final, mesmo as relacionadas com aspectos históricos, assuntos periféricos, e até as meramente incidentais. Todas estas referências são 
enumeradas, e citadas, com uma cifra arábica entre colchetes. Dentre as referências especificamente técnicas, os artigos são referidos com cifras em "roman" [4], e os livros em negrito [4]. Existem referências de caracter histórico ou incidental, incluidas para complementar o texto. Estas são referidas em "slanted" [4] ou em itálico [4] sendo estas últimas as que, por diversos motivos, não foram confrontadas pelo autor.

$\mathrm{O}$ autor agradece às pessoas e instituições sem o concurso das quais a realização deste trabalho teria sido inviável: ao $\mathrm{CNPq}$, pelo apoio financeiro durante os quatro anos de bolsa, como assim também ao Projeto PRONEX, especialmente na pessoa do seu chefe, o Prof. Dr. Walter F. Wreszinski, pelo auxílio financeiro durante a conclusão deste trabalho. Expressa também o seu agradecimento, com a mais profunda admiração e respeito, ao Prof. Dr. João Carlos Alves Barata pela sua segura, paciente e confiante orientação. Finalmente, mas não por menor merecimento, a Edith Cuello, María Alejandra Auil e Maria Salete Magnoni, mãe, irmã e dileta companheira, respectivamente, pelo incondicional apoio e afeto. 


\section{Sumário}

Prefácio ........................ iv

Convenções e Notação . . . . . . . . . . . . . . . . . . . . ix

I Completeza Assintótica 1

1 Introdução 2

1.1 A Teoria Quântica de Campos . . . . . . . . . . . . . . . 2

1.2 Teoria do Espalhamento . . . . . . . . . . . . . . . . 7

1.3 Completeza Assintótica . . . . . . . . . . . . . . . . . . . 10

1.4 Teorias na Rede . . . . . . . . . . . . . . . . . . . . . . . 14

1.5 O Presente Trabalho . . . . . . . . . . . . . . . . . 15

2 Teoria Quântica de Campos na Rede $\quad 16$

2.1 Notação, Definições, Fatos Básicos . . . . . . . . . . . . . . . . 16

2.2 O Campo Livre na Rede . . . . . . . . . . . . . . . . . . . . . . . . . . . . . . 22

2.3 Teoria do Espalhamento na Rede . . . . . . . . . . . . . . . 24

3 Completeza Assintótica 30

3.1 Introdução . . . . . . . . . . . . . . . . . . . . . 30

3.2 O Núcleo de Bethe-Salpeter na Rede . . . . . . . . . . . . . . . . 33

3.3 Relação entre Resolvente e Espectro . . . . . . . . . . . . . . . . . . . 42

3.4 Propriedades de Regularidade dos Operadores $R_{0}(k)$ e $K(k)$. . . . 46

Apêndice à Seção 3.4: Detalhes Técnicos . . . . . . . . . . . . . 56

3.5 O Espectro de Energia . . . . . . . . . . . . . . . . . . . 59

3.6 Ausência de Estados Ligados . . . . . . . . . . . . . . . . . . . . 60

3.7 O Caso de Dimensão $1+1 \ldots \ldots$. . . . . . . . . . . . . . . . 64

3.8 O Caso de Momento Não-Nulo . . . . . . . . . . . . . . . . . . 68 
II O Modelo de Ising na Região de Altas Temperaturas 73

4 Expansão em Polímeros 74

4.1 Introdução . . . . . . . . . . . . . . . . . . . . 74

4.2 O Modelo de Ising . . . . . . . . . . . . . . . . 76

4.3 Expansão em Polímeros na Região de Altas Temperaturas . . . . . . 80

5 A Função de 2 pontos 88

5.1 Decaimento Exponencial . . . . . . . . . . . . . . . . . 88

Apêndice à Seção 5.1: Decaimento Exponencial e Analiticidade. . . . 91

5.2 O Inverso da Função de 2 pontos . . . . . . . . . . . . . 92

6 As Funções de 4 pontos 96

6.1 A Parte Não-Conexa . . . . . . . . . . . . . . . . . . . . . 96

6.2 A Função de 4 pontos . . . . . . . . . . . . . . . . . . . . . . . . 99

6.3 A Parte Conexa . . . . . . . . . . . . . . . . . . . . . . . . 102

6.4 O Núcleo de Bethe-Salpeter . . . . . . . . . . . . . . . . 109

III Considerações Finais 112

7 Conclusão. Discussão e Comentários 113

8 Perspectivas, Conjeturas, Divagação 117

Referências Bibliográficas $\quad 121$

Índice Remissivo $\quad 130$

Epílogo . . . . . . . . . . . . . . . . . . . . . . 134 


\section{Convenções e Notação}

No cálculo de predicados, se $P$ e $Q$ denotam duas proposições quaisquer, convencionaremos em que

$$
P:=Q
$$

significa "P é por definição igual a $Q$ ". Em ocasiões convém manter a ordem invertendo o significado, assim

$$
P=: Q
$$

significará "Q é por definição igual a $P$ ". Também, e com menor freqüência, revelase útil denotar

$$
P:=: Q:=R
$$

ou alternativamente

$$
P:=R=: Q,
$$

significando em ambos casos " $P$ e/ou $Q$ são por definição iguais a $R$, do qual constituem notações alternativas".

O símbolo $\square$ indica o final de uma demonstração, no entanto $\square$ indica o final de uma observação, de um enunciado, ou de uma prova incompleta.

Os termos conjunto e família serão tidos como sinônimos, usados ambos para designar coleções "restritas" de objetos (por oposicão a classe que designa coleções "mais amplas" das quais os conjuntos são casos particulares).

Se $A$ e $B$ denotam dois conjuntos quaisquer, subconjuntos de um conjunto "universal" fixo $X$, se denotará:

$$
\begin{aligned}
P(A) & =\text { família de subconjuntos (ou partes) de } A \\
P F(A) & =\text { família de suconjuntos finitos de } A \\
|A| & =\text { cardinal de } A \\
A^{c} & =\text { complemento de } A \text { (relativo a } X) \\
A \backslash B & =A \cap B^{c} \\
A \triangle B & =(A \backslash B) \cap(B \backslash A) \text { ou diferença simétrica de } A \text { com } B .
\end{aligned}
$$

Denotaremos com $\mathbb{N}$ ao conjunto indutivo e bem-ordenado dos números naturais (sem o zero!). Estas duas propriedades serão usadas sob a forma de demonstrações por indução. Também denotaremos com $\mathbb{Z}$ ao anel de integridade dos números inteiros, com $\mathbb{R}$ ao corpo ordenado e completo dos números reais e com $\mathbb{C}$ ao corpo algébricamente fechado dos números complexos, em cujo caso $\operatorname{Re} z$ e $\operatorname{Im} z$ denotam 
respectivamente a parte real e imaginária de $z \in \mathbb{C}$. Não teremos ocasião de usar no presente o conjunto $\mathbb{Q}$ dos números racionais.

Se $A$ é um subconjunto de um espaço vetorial, se denotará:

$$
\begin{aligned}
& \operatorname{span} A=\text { espaço vetorial gerado por } A \\
& \overline{\operatorname{span}} A=\text { espaço vetorial fechado gerado por } A .
\end{aligned}
$$

Se $X$ e $Y$ são espaços topológicos, se denotará:

$$
\begin{aligned}
C(X, Y) & =\text { conjunto de funções contínuas de } X \text { em } Y \\
C(X) & =C(X, \mathbb{C}) \\
X^{\prime} & =\text { conjunto de funções lineares em } C(X) \text { ou espaço dual de } X
\end{aligned}
$$

considerando em $C(X)$ a estrutura métrica induzida pela norma:

$$
\|f\|_{\infty}:=\sup _{x \in X}|f(x)|
$$

quando $X$ é compacto.

Denotaremos com $\mathcal{D}\left(\mathbb{R}^{n}\right)$ o espaço de funções infinitamente diferenciáveis e de suporte compacto, e por $\mathcal{S}\left(\mathbb{R}^{n}\right)$ o espaço de Schwarz de funções rapidamente decrescentes, com ${ }^{\wedge} \mathrm{e}$ para a transformada e anti-transformada de Fourier respectivamente:

$$
\begin{gathered}
\widehat{f}(\lambda)=\frac{1}{(2 \pi)^{n / 2}} \int_{\mathbb{R}^{n}} \mathrm{e}^{-i \lambda \cdot x} f(x) d x \\
\check{f}(\lambda)=\frac{1}{(2 \pi)^{n / 2}} \int_{\mathbb{R}^{n}} \mathrm{e}^{i \lambda \cdot x} f(x) d x .
\end{gathered}
$$

Diremos que uma função, medida ou distribuição está suportada num dado conjunto, se ou seu suporte está contido em tal conjunto. Denotaremos também:

supp = suporte de funções, medidas, ou distribuições

ker $=$ núcleo ou espaço nulo de aplicações em geral

grad $=$ gradiente de funções ou operador $\nabla$.

O produto escalar num espaço de Hilbert $\mathcal{H}$ se denotará $\langle., .\rangle_{\mathcal{H}}$ convencionandose que é antilinear no primeiro argumento e linear no segundo. O subíndice fazendo menção ao espaço será dispensado quando não houver risco de confusão. Denotaremos também:

$$
\begin{aligned}
& L(\mathcal{H})=\text { conjunto de operadores lineares em } \mathcal{H} \\
& B(\mathcal{H})=\text { conjunto de operadores lineares e limitados em } \mathcal{H}
\end{aligned}
$$


considerando neste último a estrutura de espaço de Banach induzida pela norma:

$$
\|T\|_{B(\mathcal{H})}:=\sup _{\substack{x \in \mathcal{H} \\\|x\|=1}}\|T(x)\|
$$

Porém, no caso particular de dimensão finita, se denotará:

$$
\begin{aligned}
& M_{n}(\mathbb{F})=\text { conjunto de matrizes } n \times n \text { com coeficientes no anel } \mathbb{F} \\
& D_{n}(\mathbb{F})=\text { subálgebra das matrizes diagonais }
\end{aligned}
$$

(usualmente, o anel (comutativo com unidade) considerado será o corpo complexo ou o real). Neste último caso, as matrizes diagonais serão expressadas na forma:

$$
\operatorname{diag}\left(a_{1}, \ldots, a_{n}\right):=\left(\begin{array}{ccccc}
a_{1} & 0 & \ldots \ldots & 0 \\
0 & a_{2} & 0 & \ldots & 0 \\
\ldots \ldots & \ldots \ldots & \ldots \\
0 & \ldots & \ldots & 0 & a_{n}
\end{array}\right)
$$

Se $\mathcal{A}$ é uma $\mathrm{C}^{*}$-álgebra, se denotará:

$$
S_{\mathcal{A}}=\text { conjunto de estados em } \mathcal{A} \text {. }
$$

Finalmente, estas e todas as outras definições, símbolos e notações usadas no presente trabalho estarão constando num índice remissivo, no final. 


\section{Parte I}

\section{Completeza Assintótica}




\section{Capítulo 1}

\section{Introdução}

\subsection{A Teoria Quântica de Campos}

"Axiom 1: Axiomatic Field Theory is beautiful in an empty sort of way."

$$
\text { R. Haag }{ }^{1}
$$

O objetivo dos métodos rigorosos na teoria quântica de campos consiste na análise das noções gerais subjacentes a todas as teorias de campo quânticas e relativísticas. O estudo das mesmas sobre uma base axiomática começou a ser sistematizado no começo dos anos 50, na tentativa de desvendar o mistério das séries perturbativas formais que tão bem sucedidas revelaram-se na electrodinâmica quântica. O problema de combinar numa teoria de campo a Mecânica Quântica e a Relatividade ainda permanece aberto, ao menos conceitualmente, pois ainda não se dispõe de um controle não-perturbativo sobre teorias quânticas de campo não-triviais em 4 dimensões. Não existe até hoje resposta completa para a questão fundamental na qual originou-se o desenvolvimento de tais métodos, a saber: São os princípios locais da teoria quântica relativista (em 4 dimensões) compatíveis com a existência de uma matriz de espalhamento não-trivial? Contudo, consideráveis avanços têm sido feitos neste sentido nas últimas décadas.

Se bem que a moderna teoria das partículas elementares enveredou pelo caminho das teorias de calibre não-abelianas (que são generalizações diretas da eletrodinâmica quântica dos anos 40), o esquema axiomático original tem sido modificado tentando proporcionar um marco geral que contemple os resultados standard dessas teorias perturbativas. ${ }^{2}$ Novos problemas têm surgido nesta área (basicamente, pela não-localidade devido à introdução de campos não-observáveis). O estudo das

\footnotetext{
${ }^{1}$ Da sua citação em [43] p. 5.

${ }^{2}$ [91], [104]. Cf. [36] p. 374.
} 
estruturas matemáticas emergentes de tais esquemas, além do interesse intrínseco, permite estudar modelos de calibre simples (v.g. o modelo de Schwinger em duas dimensões) e interessantes, no sentido que contêm características similares às esperadas para modelos em teorias não-triviais em 4 dimensões (confinamento, quebra de simetria). ${ }^{3}$

A Teoria Quântica de Campos teve também um grande impacto, no começo dos anos 70, no estudo dos fenômenos críticos. Se bem que tais métodos já tinham sido aplicados na física nuclear e do estado sólido (na área da superfluidez e da supercondutividade surgiu a noção de quebra de simetria), a introdução do conceito de renormalização na física estatística significou uma virada de página. Basicamente, a grandes distâncias as funções de correlação de um sistema perto de um ponto crítico são descritas por uma teoria renormalizada. Esta idéia inspiradora, lançou por sua vez uma nova luz sobre o conceito de renormalização.

Muitas situações e conceitos profundos na teoria de campos têm análogos e contrapartidas na área da física do estado sólido, v.g., no efeito Kondo (regimes de confinamento e liberdade assintótica), o mecanismo de Peierls de geráção dinâmica de uma banda isolante num composto de poliacetileno (geração dinâmica de massa), o efeito Meissner dual em supercondutores de tipo II (confinamento), a liga supercondutora em altas temperaturas $\mathrm{YBa}_{2} \mathrm{Cu}_{3} \mathrm{O}_{6+x}$ onde o limite ao contínuo do modelo de Heisenberg antiferromagnetico em duas dimensões conduz ao modelo $\sigma$ não-linear (sólitons topológicos), como assim também no efeito Hall quântico, sistemas magnéticos, vórtices em supercondutores e superfluidos. ${ }^{4}$

Mudando de área, é impossível deixar de mencionar também o Teorema de Bisognano-Wichmann ${ }^{5}$ que, na sua linguagem algébrica, proporciona uma bela aplicação da Teoria de Tomita-Takesaki na física, estando na base dos estudos rigorosos sobre o efeito Hawking-Unruh. ${ }^{6}$

Uma das possivéis abordagens da Teoria Quântica de Campos tem como ponto de partida o estudo direto dos campos puramente locais. Gårding e Wightman formularam uma definição de campo quântico, propondo um conjunto de propriedades matemáticas as quais, argumentavam, toda teoria quântica de campos deveria ter. ${ }^{7}$ Estes postulados básicos, em parte altamente técnicos e abstratos, são chamados de axiomas de Gårding-Wightman, e seu estudo e suas conseqüências matemáticas são usualmente chamados de Teoria Quântica de Campos Axiomática. ${ }^{8}$ Este

\footnotetext{
${ }^{3}[104]$.

${ }^{4}$ E. C. Marino, Field Theory and Condensed Matter: Account of a Succeful Interaction, PRONEX 203 Mathematical Physics Meeting, IF-USP, São Paulo, 10 ao 12 de novembro de 1999.

${ }^{5}$ Veja-se, e.g., [44] p. 245. Cf. as referências originais [5], [6].

${ }^{6}[92]$.

${ }^{7}[108]$.

${ }^{8}$ Como referências gerais podem citar-se [77], [103], [57], [7].
} 
nome é um tanto desafortunado já que, por um lado, todas as outras abordagens são axiomáticas (neste sentido) e, por outro, pode dar a falsa impressão que o interesse principal são os axiomas em si mesmos em vez de suas conseqüências matemáticas e a construção de exemplos específicos. ${ }^{9}$ Por este motivo o assunto é chamado por vezes de Teoria Geral de Campos Quantizados.

Nos axiomas de Gårding-Wightman os campos locais são definidos com sendo basicamente "distribuições a valores em operadores" $\mathcal{S}\left(\mathbb{R}^{4}\right) \ni f \longmapsto \phi(f) \in L(\mathcal{H})$ com domínio comum invariante e denso num espaço de Hilbert $\mathcal{H}$ o qual supõese conter uma representação unitária fortemente contínua de grupo de Poincaré restrito cujo subgrupo de translações admite um único vetor invariante não-nulo $\psi_{0}$, o vácuo, e satisfaz a seguinte condição espectral: a sua medida espectral conjunta está suportada no fecho do cone positivo. ${ }^{10}$ A estas propriedades acrescentam-se invariância relativista, comutatividade local (também chamada de causalidade microscópica) e ciclicidade do vácuo. Os valores esperados no vácuo $f_{1} \otimes \cdots \otimes f_{n} \longmapsto\left\langle\psi_{0}, \phi\left(f_{1}\right) \cdots \phi\left(f_{n}\right) \psi_{0}\right\rangle$ permitem definir, via o Teorema Nuclear de Schwartz, ${ }^{11}$ distribuições temperadas $\mathcal{W}_{n}$ chamadas distribuições de Wightman. Como conseqüência da condição espectral, as distribuições de Wightman são valores de contorno de funções analíticas no tubo positivo ${ }^{12}$ chamadas de funções de Wightman.

Os resultados obtidos pela teoria axiomática, se bem que limitados pelo fato de basear-se em certo número de hipóteses especiais, podem resultar também os mais interessantes, levando em conta que partem de princípios fundamentais: invariância relativista, existência de um sistema completo de estados com energia positiva, e causalidade. O objetivo final deste estudo é uma teoria da estrutura local dos campos que permita determinar quais são as propriedades fixadas pelas hipóteses gerais contidas nos axiomas, e quais são aquelas que podem ser arbitrariamente fixadas pela escolha de uma dinâmica específica. Como no enfoque axiomático qualquer consideração particular sobre esta última é omitida, os axiomas deixarão de abranger um aspecto importante dos sistemas envolvendo campos quânticos: dificilmente será possível atingir a descrição de um tal sistema que seja estritamente axiomático e exibindo ostensivamente mecanismos dinâmicos. Por este motivo, os métodos da teoria foram complementados com elementos novos e estendidos além da esfera dos estudos estruturais genéricos para a análise de sistemas concretos e modelos (veja-se p. 7).

Existem duas reformulações equivalentes dos axiomas de Gårding-Wightman. A

\footnotetext{
${ }^{9}$ Num contexto diferente, o termo codificação é contraposto a axiomatização para referir-se a uma situação similar ([51] p. 1).

${ }^{10}$ Este último definido por: $V_{+}:=\left\{x=\left(x_{0}, x_{1}, x_{2}, x_{3}\right) \in \mathbb{R}^{4}: x_{0}^{2}-x_{1}^{2}-x_{2}^{2}-x_{3}^{2}>0 \wedge x_{0}>0\right\}$.

${ }^{11}$ Veja-se, e.g., [76] p. 141.

${ }^{12}$ Definido como sendo o conjunto $\mathbb{R}^{4 n}-i V_{+}^{n}$, onde $V_{+}^{n}:=\underbrace{V_{+} \times \cdots \times V_{+}}_{n \text { vezes }}$.
} 
primeira é do próprio Wightman, que estabeleceu um conjunto de postulados para um conjunto de distribuições temperadas $\left\{\mathcal{W}_{n}: \mathcal{W}_{n} \in \mathcal{S}^{\prime}\left(\mathbb{R}^{4 n}\right)\right\}_{n \in \mathbb{N}}$ e provou que esses postulados garantem que tais ditribuições são as distribuições de Wightman associadas com alguma (essencialmente única) Teoria Quântica de Campos satisfazendo os axiomas de Gårding-Wightman e, recíprocamente, que tais postulados são satisfeitos pelas distribuições de Wightman de uma teoria quântica de campos que satisfaz aqueles axiomas. ${ }^{13}$

Uma segunda reformulação dos axiomas de Gårding-Wightman é em termos das funções de Schwinger que são a restrição das funções de Wightman àqueles pontos do tubo estendido permutado ${ }^{14}$ que têm coordenadas espaciais reais e coordenada temporal puramente imaginária. Estas funções definem distribuições regulares satisfazendo determinadas propriedades (a positividade por reflexões dentre elas) que definem em seu conjunto os assim chamados axiomas de OsterwalderSchrader. Estes axiomas suplementados com mais uma condição de carater técnico envolvendo transformadas de Laplace permitem reconstruir a teoria de Wightman. ${ }^{15}$ Esta reformulação está relacionada com a abordagem euclidiana (veja-se p. 6).

Existem também esquemas axiomáticos cujas estruturas fundamentais são objetos diferentes dos campos puramente locais. Uma de tais abordagens enfatiza o papel dos "campos assintóticos" tendo conseqüentemente uma conexão direta com a teoria do espalhamento. Este é o formalismo LSZ de H. Lehmann, K. Symanzik e W. Zimmerman. ${ }^{16}$ Como conseqüência da teoria do espalhamento de Haag-Ruelle (veja-se p. 8), K. Hepp provou que uma versão dos axiomas LSZ é satisfeita numa teoria de Wightman com hipóteses adicionais sobre o espectro de massa. ${ }^{17} \mathrm{O}$ primeiro resultado realmente significativo no formalismo LSZ é uma fórmula explícita para a matriz $S$ em termos das distribuições de Wightman. Estas fórmulas de redução foram provadas no trabalho de Hepp. ${ }^{18}$ As fórmulas de redução são o primeiro passo na análise das propriedades de analiticidade das amplitudes de espalhamento na teoria quântica de campos axiomática.

Uma segunda abordagem envolve o uso de álgebras de Banach. Foi J. von Neumann o primeiro em sugerir o uso de álgebras de operadores lineares limitados

\footnotetext{
${ }^{13}$ Este assim chamado Teorema de Reconstrução de Wightman apareceu em [107]. Porém, este artigo não contém o equivalente do axioma de unicidade do vácuo em termos das $\mathcal{W}_{n}$, o qual foi acrescentado nos trabalhos posteriores [50] e [8].

${ }^{14}$ Definido como sendo o conjunto de todas a permutações das imagens por todas as transformações de Lorentz complexas do tubo positivo.

${ }^{15}[72],[73],[74]$.

${ }^{16}[60],[61]$.

${ }^{17}[47],[48]$. Cf. [102].

${ }^{18}$ Ibidem.
} 
para axiomatizar inicialmente a Mecânica Quântica, desenvolvendo a teoria das W*álgebras. Seu trabalho foi esclarecido e ampliado por I. Segal, quem ao longo dos anos 50 esteve à cabeça dos que propunham uma abordagem algébrica para os problemas da teoria de campos. Sem entrar em maiores detalhes, uma teoria de campo nesta abordagem está caracterizada por uma rede

$$
\Lambda \longmapsto \mathcal{A}(\Lambda)
$$

de $\mathrm{C}^{*}$-álgebras indexada por regiões abertas e limitadas $\Lambda$ do espaço-tempo e satisfazendo certas propriedades introduzidas como axiomas. ${ }^{19}$ As álgebras $\mathcal{A}(\Lambda)$ são denominadas de álgebras locais e o completamento em norma da união de todas elas recebe o nome de álgebra quase-local. R. Haag e D. Kastler formularam um conjunto de axiomas em $1964 .^{20}$ A relação destes com os de Gårding-Wightman não é direta. Intuitivamente, a álgebra $\mathcal{A}(\Lambda)$ seria a álgebra polinomial gerada pelos operadores $\phi(f)$ com supp $f \subset \Lambda$, porém isto não é rigoroso. Se os campos nos axiomas de Wightman são auto-adjuntos, então pode-se construir uma família de álgebras a partir de sua projeções espectrais, porém não fica claro se os axiomas de HaagKastler são satisfeitos neste caso por motivos técnicos, v.g., a comutatividade dos campos não implica a comutatividade das projeções espectrais. Reciprocamente, também não fica claro como reconstruir os campos a partir das álgebras locais de Haag-Kastler. Contudo, ambos conjuntos de axiomas estão estreitamente relacionados sendo de esperar que ambos sejam satisfeitos em modelos razoáveis, como tem sido o caso nos modelos em duas dimensões construidos até agora.

Uma terceira abordagem envolve a continuação analítica para o tempo imaginário, onde o grupo de Poincaré é substituido pelo grupo euclidiano. É a este nivel de continuação que as funções de Schwinger e os axiomas de Osterwalder-Schrader operam. Porém, o programa da abordagem euclidiana é mais amplo: procura-se construir campos euclidianos, ou seja, operadores cujos valores esperados sejam as funções de Schwinger. Como estas funções são simétricas por permutações arbitrárias de seus argumentos, tais operadores seriam mutuamente comutativos e, se auto-adjuntos, simultaneamente diagonalizáveis num espaço de probabilidade, em cujo caso as funções de Schwinger resultam as funções geradoras de momentos da medida de probabilidade. E. Nelson propôs um conjunto de axiomas definindo uma Teoria Quântica de Campos Euclidiana e provou que a partir de uma tal teoria é possivel construir uma outra satisfazendo os axiomas de Wightman. ${ }^{21} \mathrm{O}$ problema recíproco não foi definitivamente resolvido. ${ }^{22}$

\footnotetext{
${ }^{19}$ Entre as referências gerais para esta abordagem podem ser citadas [43], [44], [80], [58]. ${ }^{20}[46]$.

21 [66].

${ }^{22}$ Contudo, uma solução parcial pode ser encontrada em [94]. Este artigo inclui um exemplo
} 
Determinadas idéias e métodos da teoria das probabilidades, como a fórmula de Feynman-Kac, são suceptíveis de serem aplicadas nesta formulação, na construção e análise de modelos concretos e exemplos. Este estágio realizou-se na Teoria Quântica de Campos Construtiva. O ponto de partida do programa construtivo é uma teoria de campo aproximada, cuja existência seja conhecida, a partir da qual é construido um limite invariante de Lorentz quando a aproximação é removida. ${ }^{23}$ Assim que o modelo tenha sido construido, o interesse desloca-se para o estudo detalhado das suas propriedades e a maneira como estas dependem dos parâmetros envolvidos na definição do modelo. Este programa concretizou-se para um determinado número de modelos em dimensão do espaço-tempo menor que 4. Em dimensão 2 existe um conhecimento consideravelmente detalhado de vários modelos. ${ }^{24} \mathrm{Em}$ dimensão 3 também tem sido construido um modelo não-trivial. ${ }^{25}$

\subsection{Teoria do Espalhamento}

They are drawn thro'unbounded space, scatter'd upon The Void in incoherent despair.

William Blake ${ }^{26}$

Os axiomas de Gårding-Wightman não introduzem a noção de partícula, embora esta seja de crucial importância na procura de estabelecer um nexo entre a teoria e a realidade física. O principal observável na física de partículas elementares é a matriz de espalhamento ou matriz $S$, a qual descreve o resultado de experiências de espalhamento relacionando o estado das partículas antes e após a interação e pode ser expressada em termos das funções de Wightman temporalmente ordenadas (formalismo LSZ) sendo, em certo sentido, efetivamente computável. Sem entrar em maiores detalhes, diremos apenas que a formulação em termos da matriz $S$ depende essencialmente da possibilidade de definir estados assintóticos iniciais e finais, os quais são a realização de partículas livres e podem portanto ser descritos através dos seus campos livres correspondentes. Sob condições razoáveis, a teoria axiomática permite formalizar de maneira precisa tais campos assintóticos, a construção dos

satisfazendo o análogo dos axiomas de Wightman em uma dimensão mas não satisfazendo os axiomas de Nelson, o que prova que alguma estrutura adicional está presente nas teorias de campo euclidianas.

${ }^{23}$ Referências gerais: [34], [35], [36], [95], [96].

${ }^{24}[37]$, [38], [39].

${ }^{25}[56],[32]$.

${ }^{26}$ Jerusalem, Chapter I (Invocation). 
quais constitui a assim denominada Teoria de Haag-Ruelle. ${ }^{27}$ A teoria de HaagRuelle é importante, tanto quanto elegante, já que mostra conceitualmente como os campos locais estão relacionados com o espalhamento das partículas individuais. De fato, Gårding e Wightman formularam os seus axiomas em começos dos anos 50 achando prematura sua publicação entanto não existissem exemplos não-triviais. Contudo, versões preliminares destes axiomas circularam em diversos lugares, e sobre esta base a teoria do espalhamento de Haag-Ruelle foi desenvolvida. O profundo conteúdo físico e a beleza desta última foram tais, que acabaram motivando a publicação dos axiomas por Wightman e Gårding. ${ }^{28}$

Usualmente, a dinâmica interagente é obtida incialmente perturbando uma dinâmica simples que cumpre assim o rol de dinâmica livre. Porém, na Teoria Quântica de Campos (assim como também em outras situações físicas) não existe uma dinâmica não-pertubada a comparar com a dinâmica interagente. Certamente isso é esperado num contexto axiomático geral, mas também é o caso em certos modelos obtidos perturbando um campo livre de massa $m_{0} \cdot{ }^{29}$ Resulta fisicamente significativo que a massa das partículas na teoria interagente não seja $m_{0}$. Ou seja, nas teorias quânticas de campo interagentes, diferentemente das teorias clássicas, as partículas não podem escapar completamente a suas mútuas interações. Nesses casos, inicialmente são isoladas certas soluções particularmente simples do sistema interagente, para após tentar descrever o sistema interagente completo em termos das interações destas soluções simples.

A hipótese da teoria de Haag-Ruelle é a existência de um autovalor isolado $m$ do operador de massa. Certamente, esta hipótese não é viável na presença de partículas sem massa, embora uma teoria do espalhamento poda ser desenvolvida neste caso também (veja-se infra). Como resultado obtem-se subespaços $\mathcal{H}_{\text {in }}$ e $\mathcal{H}_{\text {out }}$ do espaço de Hilbert $\mathcal{H}$ dos estados físicos que representam estados assintóticos iniciais ou finais de varias partículas pelo espalhamento das partículas de massa $m$. Os espaços $\mathcal{H}_{\text {out }}$ têm uma estrutura natural de espaço de Fock, satisfazendo $\mathcal{H}_{\text {in }}=\mathcal{H}_{\text {out }}$.

Existe uma transcrição da teoria de Haag-Ruelle para a abordagem algébrica. ${ }^{30}$ A teoria do espalhamento de partículas sem massa no contexto algébrico foi desenvolvida em dois trabalhos notáveis de D. Buchholz. ${ }^{31}$ Aqui são usados alguns dos fatos da teoria de Haag-Ruelle; porém, o decaimento rápido das distribuições de Wightman truncadas não é mais válido. A idéia chave consiste em explorar o fato de que as soluções da equação de onda satisfazem uma espécie de princípio de Huygens. Um aspecto a ser enfatizado é que quando partículas sem massa estão presentes na

\footnotetext{
${ }^{27} \mathrm{Cf}$. as referências originais [42], [82]. Referências gerais: [78], [36], [7], [57].

${ }^{28} \mathrm{Na}$ já citada referência [108]. Cf. as notas de [77] à p. 113.

${ }^{29}$ Veja-se, e.g., [77] seção X.7.

${ }^{30}[45]$. Cf. [18].

${ }^{31}[14],[15]$. Para a formulação em termos dos campos locais veja-se [16].
} 
teoria, não se sabe como construir estados assintóticos para as partículas massivas. De fato, não fica claro como "separar" as partículas massivas das sem massa: em uma teoria de eletrons e fotons pode acontecer que não exista uma porção discreta do espectro de massa correspondente a um eletron, pois os eletrons aparecem inevitavelmente acompanhados por uma quantidade infinita de fotons de baixa energia. Este problema, o qual não está completamente comprendido, é chamado de problema do infravermelho ou problema das infrapartículas. Este mesmo problema pode acontecer com as partículas sem massa, em cujo caso a teoria de Buchholz não é aplicável já que estados com $H^{2}-\mathbf{P}^{2}=0(H \neq 0)$ são admissíveis nesta. Na presença de tais "infrapartículas"a interpretação da teoria em termos de partículas não pode ser obtida simplesmente a partir dos polos das funções de Green respectivas. Não existe ainda um procedimento geral por meio do qual o espectro correspondente a partículas, seções transversais, etc., possam ser determinados a partir da teoria nestes casos mais complicados.

No contexto algébrico existe uma relação entre a teoria do espalhamento e o limite para o equilibrio na mecânica estatística. Sejam $H_{0}=H_{0}^{*}$ e $V$ operadores lineares (não necessariamente limitados) num espaço de Hilbert $\mathcal{H}$. Consideremos os grupos uni-paramétricos de automorfismos em $L(\mathcal{H})$ dados por $\alpha_{t}^{0}(A):=\mathrm{e}^{i H_{0} t} A \mathrm{e}^{-i H_{0} t}$ e $\alpha_{t}(A):=\mathrm{e}^{i H t} A \mathrm{e}^{-i H t}$, onde $H:=H_{0}+V . \alpha_{t}(A)$ pode ser calculado iterativamente a partir de $\alpha_{t}^{0}(V)$ por meio de uma série convergente. ${ }^{32}$ Esta série é o ponto de partida para uma teoria geral de perturbações de automorfismos em $\mathrm{C}^{*}$-álgebras. Com efeito, se agora $\alpha_{t}^{0}$ é um grupo uni-paramétrico de automorfismos contínuo em norma em uma $\mathrm{C}^{*}$-álgebra $\mathcal{A}$ e $V \in \mathcal{A}$, formalmente a mesma série converge para um outro grupo uniparamétrico de automorfismos contínuo em norma que denotaremos $\alpha_{t}$. Além disso, se o $\alpha_{t}^{0}$ é unitariamente implementado numa dada representação o $\alpha_{t}$ também o é na mesma representação, e vice-versa. Pode-se provar também que se

$$
t \longmapsto\left\|\left[\alpha_{t}^{0}(V), A\right]\right\| \in L^{1}
$$

para cada $A$ num subespaço denso de $\mathcal{A}$, então para cada $A \in \mathcal{A}$ existem os limites $\lim _{t \rightarrow \pm \infty} \alpha_{-t} \alpha_{t}^{0}(A)$ e os $w_{ \pm}:=\lim _{t \rightarrow \mp \infty} \alpha_{-t} \alpha_{t}^{0}$ são morfismos injetores em $\mathcal{A}$ satisfazendo:

$$
w_{ \pm} \alpha_{t}^{0}=\alpha_{t} w_{ \pm}
$$

No caso particular em que $\alpha_{t}^{0}$ é o automorfismo de translações temporais num sistema gás quântico na rede e $V$ é um observável local, (1.2.1) é plausível para cada $A$ observável local. Se $\omega$ é um estado invariante por $\alpha_{t}$, então $\omega\left(\alpha_{t}^{0}().\right)=\omega\left(\alpha_{-t} \alpha_{t}^{0}().\right) \rightarrow$ $\omega\left(w_{ \pm}().\right)$e, por (1.2.2) $\omega \circ w_{ \pm}$são estados invariantes por $\alpha_{t}^{0}$. Ou seja, todo estado

\footnotetext{
${ }^{32}[78]$ p. 383.
} 
invariante pela dinâmica localmente perturbada que evolui segundo a dinâmica livre aproxima-se para um estado invariante por esta última. Mais ainda, sob supostos adicionais, se $\omega$ é um estado $\beta$-KMS para $\alpha_{t}$, então $\omega \circ w_{ \pm}$são estados $\beta$-KMS para $\alpha_{t}^{0}$. Este exemplo da teoria do espalhamento em $\mathrm{C}^{*}$-álgebras e sua relação com a mecânica estatística é devido a D. Robinson. ${ }^{33}$ Alguns dos aspectos tratados por Robinson já tinham sido abordados por K. Hepp no estudo do efeito Kondo. ${ }^{34} \mathrm{Fi}-$ nalmente, em alguns casos a condição (1.2.1) é restritiva demais e $\alpha_{-t} \alpha_{t}^{0}$ resulta apenas fracamente convergente. Em tais casos resulta mais natural supor que $\mathcal{A}$ é uma álgebra de von Neumann. Uma abordagem deste tipo é usada na análise de alguns aspectos no espalhamento por potenciais de longo alcance. A teoria do espalhamento em termos de automorfismos desempenha também um papel no estudo de propriedades espectrais de hamiltonianos em certos modelos discretizados na teoria quântica de campos. ${ }^{35}$

\subsection{Completeza Assintótica}

The fist step of the proof is to wait.

\section{Enss ${ }^{36}$}

A questão da completeza assintótica é de saber se $\mathcal{H}_{\text {in }}=\mathcal{H}$. Sobre uma base fenomenológica, é de esperar ter completeza numa teoria fisicamente razoável. Ou seja, é de esperar que cada estado de un sistema físico poda ser visto bem como descrevendo partículas (incluindo seus estados ligados) bem como decaindo num tal estado no transcurso do tempo. Implicitamente nesta idéia está o fato de que a dinâmica livre não tem estados ligados. Um exemplo onde a completeza falha é um espaço de Fock restrito a vetores com um número par de partículas. Estados de uma única partícula não podem existir em tal teoria. Quando tais estados aparecem é de esperar que o espaço de Hilbert original $\mathcal{H}$ deva ser ampliado aos fins de obter uma descrição física adequada. Desde o ponto de vista da teoria de Haag-Ruelle (i.e., físico) tais estados são patológicos. Um outro exemplo artificial é o seguinte: suponha-se ter sido construida uma teoria interagente da eletrodinâmica quântica que é assintoticamente completa. Uma tal teoria restrita ao campo eletromagnético e ao subespaço cíclico por ele gerado não pode ser assintoticamente completa pois o espaço de Hilbert poderia conter estados de pares de partículas eletron-pósitron sem

\footnotetext{
${ }^{33}[81]$. Este artigo contém os detalhes dos resultados citados acima como assim também cálculos para alguns modelos específicos.

${ }^{34}[49]$

${ }^{35}[78]$ p. 384.

${ }^{36}$ Citado em [78] p. 331.
} 
conter os estados de uma partícula correspondentes, os quais não estão acoplados ao vácuo pelo campo eletromagnético pelo fato de não ter carga. Existem fortes indícios que fenômenos similares aconteriam no caso de certos campos bosônicos auto-interagentes em duas dimensões onde acredita-se que certos estados de duas partículas (pares de soliton e anti-soliton) estão acoplados ao vácuo pelo campo bosônico embora os estados de uma partícula correspondentes não. ${ }^{37}$

Contudo, é uma questão matemática profunda e ainda em aberto estabelecer a completeza assintótica, ainda para os exemplos concretos existentes na teoria quântica de campos. Sabe-se que modelos de campos livres generalizados não são assintoticamente completos. ${ }^{38}$ Existem também resultados parciais na teoria $P(\varphi)_{2}$ fracamente acoplada na região de baixas energias.

Quando o polinômio da interação $P(\varphi)$ é par são distinguidos os espaços $\mathcal{H}_{\text {par }}$ e $\mathcal{H}_{\text {impar }}$ gerados pelos produtos de um número par ou ímpar dos operadores do campo, respectivamente. ${ }^{39}$ Se $A \subseteq \mathbb{R}$ é um intervalo, denotando com $E$ a família espectral do Hamiltoniano, definimos $\mathcal{H}^{A}:=E(A) \mathcal{H}$ e denotamos:

$$
\begin{aligned}
\mathcal{H}_{\dagger}^{A} & :=\mathcal{H}^{A} \cap \mathcal{H}_{\dagger} \\
\mathcal{H}_{\dagger, \ddagger}^{A} & :=\mathcal{H}_{\dagger}^{A} \cap \mathcal{H}_{\ddagger}
\end{aligned}
$$

onde $\dagger=$ par ou impar e $\ddagger=$ in ou out. Glimm, Jaffe e Spencer ${ }^{40}$ estabeleceram que:

$$
\mathcal{H}_{\text {in,par }}^{[0,2(m-\epsilon))}=\mathcal{H}_{\text {par }}^{[0,2(m-\epsilon))}=\mathcal{H}_{\text {out }, \text { par }}^{[0,2(m-\epsilon))}
$$

se $\lambda$ é suficientemente pequeno. Este resultado foi generalizado por Spencer para o caso em que $\lambda$ é menor que um certo valor crítico. ${ }^{41}$ Também Glimm e Jaffe ${ }^{42}$ estabeleceram condições suficientes para que:

$$
\mathcal{H}_{\text {in,impar }}^{[0,3(m-\epsilon))}=\mathcal{H}_{\text {impar }}^{[0,3(m-\epsilon))}=\mathcal{H}_{\text {out }, \text { impar }}^{[0,3(m-\epsilon))} .
$$

Esta condição, que é basicamente decaimento exponencial com a separação dos seus argumentos com taxa $3 m$ da parte conexa da função de 6 pontos, é predita para a interação $\varphi^{4}$ pela primeira ordem não-nula da teoria de perturbações, ao menos para acoplamento fraco. Nos dois casos anteriores o operador de massa tem os autovalores 0 (correspondente ao vácuo) e $m$ (estados de uma partícula) e nenhum outro espectro nos intervalos respectivos.

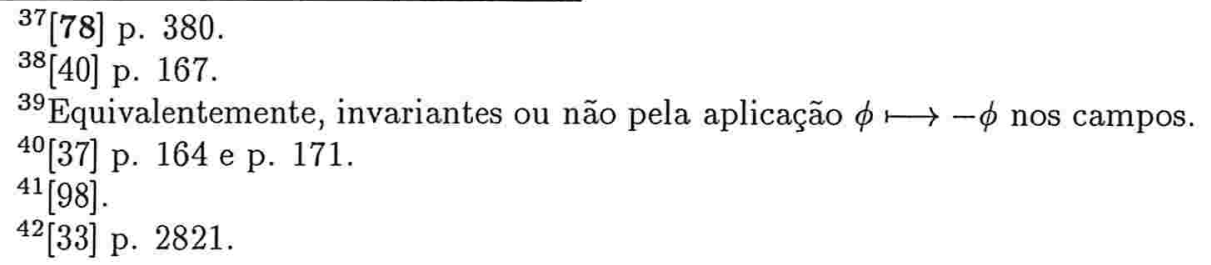


O primeiro trabalho envolvendo o espectro contínuo (que corresponde intuitivamente aos estados assintóticos) é o de Spencer e Zirilli. ${ }^{43}$ Sob hipóteses de analiticidade do núcleo de Bethe-Salpeter de duas partículas no espaço de energia-momento ${ }^{44}$ é verificado que:

$$
\mathcal{H}_{\text {in,par }}^{[2(m-\epsilon), 4(m-\epsilon))}=\mathcal{H}_{\text {par }}^{[2(m-\epsilon), 4(m-\epsilon))}=\mathcal{H}_{\text {out }, \text { par }}^{[2(m-\epsilon), 4(m-\epsilon))} .
$$

Em particular, o espectro do operador de massa é discreto e de multiplicidade finita abaixo de $2 m$ e absolutamente contínuo e de multiplicidade 1 por cima, se o o coeficiente de $\varphi^{4}$ é positivo.

O sinal deste coeficiente determina a presença ou ausência de estados ligados de duas partículas. Se é positivo não existe espectro abaixo de $2 m$. Se é negativo existe exatamente um estado ligado de duas partículas no intervalo $(m, 2 m)$. Além disso, não existe outro espectro até $3(m-\epsilon) .{ }^{45}$

Se os coeficientes de $\varphi^{4}$ e $\varphi^{6}$ são ambos negativos pode-se provar a existência de um estado ligado de três partículas. Mais especificamente, se o coeficente de $\varphi^{4}$ é negativo (ou seja, admitindo a existência de estados de duas partículas) então: se o coeficiente de $\varphi^{6}$ é positivo não existem estados ligados de três partículas entanto que se é negativo existe exatamente um. Além disso, não existe nenhum outro espectro no subespaço ímpar no intervalo $(m, 3 m){ }^{46}$

Assumindo que não existem estados de duas partículas foi provado ${ }^{47}$ também que:

$$
\mathcal{H}_{\text {in,impar }}^{(3 m+a(\lambda), 4 m-b(\lambda))}=\mathcal{H}_{\text {impar }}^{(3 m+a(\lambda), 4 m-b(\lambda))}=\mathcal{H}_{\text {out }, \text { impar }}^{(3 m+a(\lambda), 4 m-b(\lambda))},
$$

onde $a$ e $b$ são funções positivas que tendem para zero quando $\lambda \rightarrow 0$, embora o resultado desejado (e não obtido) fosse:

$$
\mathcal{H}_{\text {in,impar }}^{[3(m-\epsilon), 5(m-\epsilon))}=\mathcal{H}_{\text {impar }}^{[3(m-\epsilon), 5(m-\epsilon))}=\mathcal{H}_{\text {out }, \text { impar }}^{[3(m-\epsilon), 5(m-\epsilon))} .
$$

Todos estes últimos resultados baseiam-se na técnica de "perturbações compactas" do trabalho de Spencer e Zirilli, ${ }^{48}$ constituindo, aliás, uma espécie de saga deste último. Naturalmente, acreditou-se durante um certo tempo que resultados ainda mais gerais poderiam ser obtidos de maneira similar. Dificultades importantes, porém, obstaculizaram este programa. ${ }^{49}$ D. Iagolnitzer propôs uma análise mais heurística na qual foram conjeturadas equações de estrutura, envolvendo núcleos

\footnotetext{
${ }^{43}[101]$.

${ }^{44}$ Previamente estabelecidas e provadas em [99]. Cf. [100].

${ }^{45}[25],[26]$.

${ }^{46}[68]$.

${ }^{47}[21]$.

${ }^{48}$ Op. Cit.. Veja-se na seção 3.1, p. 32 , para uma breve descrição deste método.

${ }^{49}$ [32], [21], [24].
} 
irredutíveis mais gerais que são generalizações, em cada intervalo de energias, das séries de Neumann da parte conexa da função de 4 pontos em termos de núcleo de Bethe-Salpeter, e das quais sistemas adequados de equações integrais podem ser derivados em última instância. Mais ainda, acrescentando algumas conjeturas de caráter matemático, prova-se que aqueles conduzem, ao menos formalmente (para teorias sem estados ligados), às relações de completeza assintótica em intervalos de energias em geral, como também a resultados adicionais sobre a estrutura analítica das funções de Green de várias partículas e amplitudes de colisão. ${ }^{50}$

Finalmente, não é possível deixar de mencionar o tratamento da completeza assintótica na abordagem algébrica, originalmente proposto por Haag e Araki. ${ }^{51}$ Seja $A \in \mathcal{A}(\Lambda)$ um observável local. Para cada elemento $U(x)$ do subgrupo de translações do espaço-tempo, denotamos:

$$
A(x):=U(x) A U(x)^{-1} .
$$

Seja agora $f \in \mathcal{D}\left(\mathbb{R}^{4}\right)$ com $\widehat{f} \in \mathcal{D}\left(\mathbb{R}^{4}\right)$ e supp $\widehat{f} \subset \bar{V}_{+}^{c}$. Estas propriedades do suporte mais a condição espectral permitem provar que o operador definido por:

$$
B:=\int A(x) f(x) d x
$$

satisfaz $B \psi_{0}=0$. Assim, o operador $C:=B^{*} B$ pode ser interpretado como uma espécie de detector: é um observável quase-local sensitivo às exitações do vácuo. Tais operadores geram uma *álgebra $\mathcal{C}$ denominada álgebra de detectores. Estes autores provaram que para cada $h \in \mathcal{D}\left(\mathbb{R}^{4}\right)$ a media espacial de $C$ ao tempo $t$ :

$$
C_{h}(t):=\int h\left(\frac{\mathbf{x}}{t}\right) C((t, \mathbf{x})) d \mathbf{x}
$$

converge fracamente num conjunto denso de vetores quando $t \rightarrow \mp \infty$. A completeza assintótica é expressada neste contexto pela propriedade de que a álgebra de von Neumann $\mathcal{C}_{\text {in }}$ gerada por tais limites fracos mais o operador identidade possua um conjunto completo de observáveis que comutam. D. Buchholz propôs ao longo destas linhas um esquema generalizado que contemple também a existência de infrapartículas. ${ }^{52}$

\footnotetext{
${ }^{50}$ [52], [53], [54].

${ }^{51}[45]$.

$52[17]$.
} 


\subsection{Teorias na Rede}

As teorias de calibre na rede foram estudadas primeiramente por $\mathrm{F}$. Wegner, ${ }^{53}$ sob um nome diferente, para o grupo de calibre $\mathbb{Z}_{2}$. Este autor estava interessado em generalizações do modelo de Ising possuindo transições de fase sem um parâmetro de ordem local. Poucos anos depois, K. Wilson ${ }^{54}$ introduziou uma classe um tanto mais geral de teorias de calibre na rede procurando comprender o confinamento permanente dos quarks. A partir de então, constituiu-se em um campo de enorme atividade, no qual foi produzida grande quantidade de resultados fisicamente relevantes, fruto basicamente da observação que a aproximação na rede transforma teorias de calibre em uma espécie de mecânica estatística clássica e questões interessantes podem ser analisadas com as técnicas amplamente desenvolvidas desta última área. A aproximação na rede é também importante por outro motivo: fornece uma ferramenta para a construção de modelos no contínuo. De fato, revelou-se o caminho mais bem sucedido neste propósito. Foram construidos, v.g., uma teoria da eletrodinâmica quântica em duas dimensões $\left(\mathrm{QED}_{2}\right)$ e um modelo de Higgs (modelo de Landau-Ginzburg) bidimensional no qual os axiomas de Wightman têm sido verificados. ${ }^{55}$

O maior problema físico que pode ser estudado no contexto das teorias de calibre na rede ainda é aquele que originalmente motivara a Wilson a introduzi-las: o confinamento permanente dos quarks a temperatura zero. Apesar de todos o esforços até agora investidos, o confinamento ainda não foi provado, nem sequer no sentido do critério de Wilson, em modelos não-abelianos em 4 dimensões com acoplamento arbitrário. Porém, muitos resultados parciais foram obtidos, dando suporte à idéia que o vácuo de uma teoria confinante se parece com um superconductor magnético confinando o campo elétrico da cor em tubos entre as cargas por meio de um efeito Meissner "dual", produzindo assim uma força entre elas que é essencialmente independente das suas distâncias.

A teoria do espalhamento na rede foi desenvolvida por J. C. A. Barata e K. Fredenhagen ${ }^{56}$ ao longo das mesmas líneas da teoria de Haag-Ruelle, estabelecendo ademais fórmulas de redução para a matriz $S$ na rede. ${ }^{57}$ Esta teoria será considerada com maior detalhe na seção 2.3. Outros resultados e trabalhos relevantes serão citados oportunamente ao longo dos próximos capítulos, tentando favorecer assim a sua aparição dentro do contexto estimado como mais apropriado à sua inserção.

\footnotetext{
${ }^{53}[106]$.

${ }^{54}$ [109].

$55[91]$.

${ }^{56}[1],[4]$.

57[2], [3].
} 


\subsection{O Presente Trabalho}

O presente trabalho desenvolve basicamente um adaptação ao contexto da rede de certos resultados obtidos por Spencer, [99], e Spencer e Zirilli, [101], para modelos $P(\varphi)_{2}$ fracamente acoplados, constituindo-se portanto em nada mais do que um relatório do atual estado de ignorância (principalmente do autor) no campo da completeza assintótica.

No primeiro dos trabalhos citados, é provado o decaimento exponencial dos núcleos de uma e duas partículas, com a conseguinte analiticidade no espaço de energia-momento. Aqui é usada a expansão em polímeros para altas temperatu$\operatorname{ras}^{58}$ e a técnica de "desacoplamento por hiperplanos", como convencionaremos em denominá-la apenas para referência, que permite provar que algumas derivadas adequadamente avaliadas das funções de $n$ pontos são nulas. Com outra terminologia, prova-se que o núcleo de 1 partícula é 2-irredutível e o núcleo de 2 partículas ou núcleo de Bethe-Salpeter é 3-irredutível. ${ }^{59}$. A técnica de descoplamento por hiperplanos foi introduzida para o estudo de modelos na rede nos trabalhos de R. Schor. ${ }^{60}$

No segundo de tais trabalhos, a analiticidade do núcleo de Bethe-Salpeter e usada como ponto de partida para a análise do espectro do operador de massa na região de duas partículas. Em particular, assumindo adicionalmente que o núcleo de Bethe-Salpeter está dado em primeira ordem da teoria de perturbações por uma função $\delta$ repulsiva, ${ }^{61}$ segue a completeza assintótica nesta região. Aqui é usada a técnica de "perturbações compactas", como convencionaremos em denominá-la apenas para referência. Se bem esta última condição sobre o núcleo de Bethe-Salpeter é característica específica das interações do modelo $P(\varphi)_{2}$, resulta interessante destacar que boa parte das técnicas usadas neste segundo artigo, embora limitadas, não dependem do modelo. Este tipo de abordagem foi usado também para a análise de estados ligados em modelos de teorias na rede. ${ }^{62}$

No capítulo 3 da primeira das três partes em que o presente trabalho é dividido, são estabelecidas hipóteses gerais sobre a função de 2 pontos e o núcleo de BetheSalpeter para teorias na rede, sendo mostrado como é que estas se articulam dando lugar à completeza assintótica.

$\mathrm{Na}$ segunda parte, estas hipóteses são verificadas para um modelo em particular, a saber, o modelo de Ising na região de altas temperaturas, mediante a técnica de "desacoplamento por hiperplanos". A última parte é constituida apenas por comentários sobre as duas primeiras.

\footnotetext{
${ }^{58}$ Previamente provada para tais modelos em [37], [38].

${ }^{59} \mathrm{Para}$ as definições de irredutibilidade, veja-se, v.g., [20] p. 106.

${ }^{60}[84]$, [85]. Cf. [69], [70].

${ }^{61} \mathrm{O}$ que também é provado em [99].

${ }^{62}$ [90], [28]. Cf. [25], [26].
} 


\section{Capítulo 2}

\section{Teoria Quântica de Campos na Rede}

\subsection{Notação, Definições, Fatos Básicos}

O conjunto $\mathbb{Z}^{d+1}$ será chamado de rede (ou "a rede") e seus elementos serão ditos os sítios na rede. Se $S$ é um conjunto finito ${ }^{1}$ topologizado discretamente e $\Lambda \subseteq \mathbb{Z}^{d+1}$ consideraremos $S^{\Lambda}:=\{\sigma: \Lambda \longmapsto S\}$ com a topologia produto, cujos elementos serão chamados de configurações em $\Lambda$ ou simplesmente "configurações". Se $x \in \mathbb{Z}^{d+1}$, define-se a projeção na coordenada $x$ como a função $p_{x}$ dada por:

$$
p_{x}(\sigma):=\sigma(x) \forall \sigma \in S^{\Lambda} .
$$

Observe que $p_{x} \in C\left(S^{\Lambda}\right) \forall \Lambda: x \in \Lambda$. De fato, a topologia produto é, por definição, a menor topologia em $S^{\Lambda}$ tal que todas as projeções $p_{x}$ são continuas. Para cada $A \in P F\left(\mathbb{Z}^{d+1}\right)$ define-se:

$$
p_{A}:= \begin{cases}\prod_{x \in A} p_{x} & \text { se } A \neq \emptyset \\ \mathbb{I} & \text { se } A=\emptyset\end{cases}
$$

onde $\mathbb{I}$ denota a função identicamente igual a $1 \mathrm{em} S^{\Lambda}$.

\subsubsection{Observações:}

(a) $S^{\Lambda}$ é um espaço topológico compacto. ${ }^{2}$ Considerando a rede com a topologia discreta (relativa de $\mathbb{R}^{d+1}$ ) e $\Lambda \subseteq \mathbb{Z}^{d+1}$ com a relativa, $S^{\Lambda}$ não é senão $C(\Lambda, S)$.

\footnotetext{
${ }^{1}$ Esta última condição é dispensável para muitas das considerações a seguir.

${ }^{2}$ Para isto basta apenas que $S$ seja compacto (Veja-se, e.g., [76] p. 100), o que acontece, v.g., se é finito e discreto como em nosso caso.
} 
Quando $\Lambda$ é finito, $S^{\Lambda}$ é discreto, pois $\{\sigma\}=\bigcap_{x \in \Lambda} p_{x}^{-1}(\sigma(x))$. Em geral, uma vizinhança aberta de uma $\sigma \in S^{\Lambda}$ está determinada por (uma quantidade enumerável de) funções que coincidem com $\sigma$ numa quantidade finita de sítios.

(b) Pelo ponto (a) anterior, quando $\Lambda$ é finito, dada $\sigma \in S^{\Lambda}$, a aplicação $\delta_{\sigma}$ : $S^{\Lambda} \longmapsto \mathbb{C}$ dada por:

$$
\delta_{\sigma}\left(\sigma^{\prime}\right):= \begin{cases}1 & \text { se } \sigma^{\prime}=\sigma \\ 0 & \text { se } \sigma^{\prime} \neq \sigma\end{cases}
$$

está em $C\left(S^{\Lambda}\right)$, e assim qualquer $f \in C\left(S^{\Lambda}\right)$ pode ser escrita como $f=$ $\sum_{\sigma \in S^{\Lambda}} f(\sigma) \delta_{\sigma}$. Em particular, $C\left(S^{\Lambda}\right)$ é um espaço vetorial de dimensão $\left|S^{\Lambda}\right|=$ $|S|^{|\Lambda|}=: r$. No caso $|S|=2$ (v.g., quando $S=\{1,-1\}$ como no modelo de Ising) é simples provar que $C\left(S^{\Lambda}\right)=\operatorname{span}\left\{p_{A}: A \subseteq \Lambda\right\}$ como espaço vetorial, mas não como algebra. De fato, escrevendo neste caso $f=\sum_{A \in P F(\Lambda)} c_{A} p_{A}$ as aplicações de $C\left(S^{\Lambda}\right)$ em $\mathbb{C}^{r}$ dadas por:

$$
\begin{gathered}
f \longmapsto\left(f\left(\sigma_{1}\right), \ldots, f\left(\sigma_{r}\right)\right) \\
f \longmapsto\left(c_{A_{1}}, \ldots, c_{A_{r}}\right)
\end{gathered}
$$

são contínuas e preservam a estrutura de espaço vetorial mas a relação com o produto de álgebra não é clara. Aliás, em $\mathbb{C}^{r}$ não existe em geral uma definição natural de produto. Isto último pode ser realizado representando $\mathbb{C}^{r}$ em $D_{r}(\mathbb{C})$ como conjunto de matrizes diagonais via a aplicação dada por:

$$
\left(a_{1}, \ldots, a_{r}\right) \longmapsto \operatorname{diag}\left(a_{1}, \ldots, a_{r}\right) .
$$

As operações algébricas em $C\left(S^{\Lambda}\right)$ correspondem, por definição, às operações matriciais usuais em $D_{r}(\mathbb{C})$. Observe que o produto escalar de dois vetores em $\mathbb{C}^{r}$ corresponde a tomar o traço do produto de uma das matrizes vezes a matriz adjunta (neste caso, simplesmente a conjugada complexa) da outra. Esta caracterização é util para descrever estados em $C\left(S^{\Lambda}\right)$ via estados em $\mathbb{C}^{r}$ sendo estes últimos determinados, pelo lema de Riesz, ${ }^{3}$ como produtos escalares, ou então alternativamente, como traços em $D_{r}(\mathbb{C})$ (Veja-se seção 4.2$, p. 77$)$.

(c) Se com $\mathcal{P}(\Lambda)$ denotamos a álgebra fechada gerada por $\left\{p_{A}: A \subseteq \Lambda\right\}$, como é obvio que este último conjunto separa pontos em $S^{\Lambda}$, aplicando o teorema de Stone-Weirstrass, ${ }^{4}$ temos que $\mathcal{P}(\Lambda)=C\left(S^{\Lambda}\right)$ (no caso em que $S \subseteq \mathbb{R}$. No caso geral temos que acrescentar os complexos conjugados das $p_{A}$ ). Quando $S=\{1,-1\}$ basta apenas tomar o espaço vetorial gerado, pois neste caso $p_{A} p_{B}=p_{A \triangle B}$.

\footnotetext{
${ }^{3}$ Veja-se, e.g., [76] p. 43.

${ }^{4}$ Veja-se, e.g., [76] pp. 103-104.
} 
(d) No presente contexto, a álgebra $C\left(S^{\mathbb{Z}^{d+1}}\right)$ pode ser pensada como sendo de natureza indutiva. Com efeito, $P F\left(\mathbb{Z}^{d+1}\right)$ é um conjunto dirigido pela inclusão, ou seja, dizendo que $\Lambda_{2}$ segue em ordem a $\Lambda_{1}$ se e somente se $\Lambda_{2} \supseteq \Lambda_{1}$, então $\supseteq$ é uma ordem parcial (i.e., uma relação reflexiva, antisimétrica e transitiva) filtrante em $P F\left(\mathbb{Z}^{d+1}\right)$ e $\left\{C\left(S^{\Lambda}\right)\right\}_{\Lambda \in P F\left(\mathbb{Z}^{d+1}\right)}$ é uma família de $C^{*}$-álgebras com identidades $\mathbb{I}_{\Lambda}$ respectivamente. Se $\Lambda_{2} \supseteq \Lambda_{1}$ define-se $\Phi_{\Lambda_{2}, \Lambda_{1}}: C\left(S^{\Lambda_{1}}\right) \longmapsto$ $C\left(S^{\Lambda_{2}}\right)$ por:

$$
\Phi_{\Lambda_{2}, \Lambda_{1}}(f)(\sigma):=f\left(\left.\sigma\right|_{\Lambda_{1}}\right) \forall \sigma \in S^{\Lambda_{2}}
$$

É imediato que:

- $\Phi_{\Lambda_{2}, \Lambda_{1}}$ é $^{*}$-morfismo injetor e isométrico.

$-\Phi_{\Lambda_{2}, \Lambda_{1}}\left(\mathbb{I}_{\Lambda_{1}}\right)=\mathbb{I}_{\Lambda_{2}}$

$-\Lambda_{3} \supseteq \Lambda_{2} \supseteq \Lambda_{1} \Longrightarrow \Phi_{\Lambda_{3}, \Lambda_{2}} \Phi_{\Lambda_{2}, \Lambda_{1}}=\Phi_{\Lambda_{3}, \Lambda_{1}}$

de onde:

$$
\lim _{\Lambda}\left\{C\left(S^{\Lambda}\right) ; \Phi_{\Lambda_{2}, \Lambda_{1}}: \Lambda_{2} \supseteq \Lambda_{1}\right\} \text { é *-isomorfo a } C\left(S^{\mathbb{Z}^{d+1}}\right) .
$$

Com efeito, denotando $\stackrel{\circ}{\mathcal{P}}(\Lambda)$ a álgebra gerada por $\left\{p_{A}: A \subseteq \Lambda\right\}$ então:

$$
\begin{aligned}
& \stackrel{\circ}{\mathcal{P}}\left(\mathbb{Z}^{d+1}\right) \subseteq \bigcup_{\Lambda \in P F\left(\mathbb{Z}^{d+1}\right)} \stackrel{\circ}{\mathcal{P}}(\Lambda) \subseteq \bigcup_{\Lambda \in P F\left(\mathbb{Z}^{d+1}\right)} \mathcal{P}(\Lambda) \\
& =\bigcup_{\Lambda \in P F\left(\mathbb{Z}^{d+1}\right)} C\left(S^{\Lambda}\right) \subseteq \overline{\bigcup_{\Lambda \in P F\left(\mathbb{Z}^{d+1}\right)} C\left(S^{\Lambda}\right)} \\
& \Longrightarrow C\left(S^{\mathbb{Z}^{d+1}}\right) \subseteq \bigcup_{\Lambda \in P F\left(\mathbb{Z}^{d+1}\right)} C\left(S^{\Lambda}\right) .
\end{aligned}
$$

Por outro lado:

$$
\begin{aligned}
\stackrel{\circ}{\mathcal{P}}(\Lambda) \subseteq \stackrel{\circ}{\mathcal{P}}\left(\mathbb{Z}^{d+1}\right) \subseteq \mathcal{P}\left(\mathbb{Z}^{d+1}\right) \Longrightarrow & C\left(S^{\Lambda}\right) \subseteq C\left(S^{\mathbb{Z}^{d+1}}\right) \forall \Lambda \\
& \Longrightarrow \bigcup_{\Lambda \in P F\left(\mathbb{Z}^{d+1}\right)} C\left(S^{\Lambda}\right) \subseteq C\left(S^{\mathbb{Z}^{d+1}}\right) .
\end{aligned}
$$

Portanto:

$$
C\left(S^{\mathbb{Z}^{d+1}}\right)=\overline{\bigcup_{\Lambda \in P F\left(\mathbb{Z}^{d+1}\right)} C\left(S^{\Lambda}\right)}
$$

e o afirmado segue de 1.23 .3 em [83] p. 72. 
Cada $\alpha$ automorfismo de $\mathbb{Z}^{d+1}$ induz naturalmente um outro em $S^{\Lambda}$ o qual por sua vez induz um outro em $C\left(S^{\Lambda}\right)$. Com efeito, se $\sigma \in S^{\Lambda}$ então $\sigma \circ \alpha \in S^{\alpha^{-1}(\Lambda)}$. Agora, se $f \in C\left(S^{\Lambda}\right)$ define-se $\alpha f \in C\left(S^{\alpha(\Lambda)}\right)$ por $(\alpha f)(\sigma):=f(\sigma \circ \alpha)$. Dois de tais automorfismos serão particularmente considerados, a saber: as translações em $x \in \mathbb{Z}^{d+1}$ :

$$
\tau_{x}: y \longmapsto y+x
$$

e as reflexões nos hiperplanos $\left\{x \in \mathbb{R}^{d+1}: x_{i}=a\right\}$ com $a \in \frac{1}{2} \mathbb{Z}$ :

$$
\left(\theta_{i, a}(x)\right)_{j}:=\left\{\begin{array}{ll}
x_{j} & \text { se } j \neq i, \\
2 a-x_{i} & \text { se } j=i
\end{array} \quad j=0,1, \ldots, d .\right.
$$

2.1.2 Observações: Resulta simples provar que:

(a) $\tau_{x_{1}} \tau_{x_{2}}=\tau_{x_{1}+x_{2}}$, de onde: $\tau_{x}^{-1}=\tau_{-x}$.

(b) $\theta_{i, a} \tau_{x}=\tau_{x} \theta_{i, a-x_{i}}=\tau_{\theta_{i, 0}(x)} \theta_{i, a}$.

Se chamamos $e_{n}(n=0,1, \ldots, d)$ os vetores da base canônica de $\mathbb{Z}^{d+1}$ e denotamos $\tau_{n}:=\tau_{e_{n}}$ e $\theta:=\theta_{0,0}$ temos que:

(c) $\tau_{x}=\prod_{i=0}^{d} \tau_{i}^{x_{i}}$.

(d) $\theta \tau_{0}=\tau_{0}^{-1} \theta$.

(e) $\theta \tau_{n}=\tau_{n} \theta$ para $n=1, \ldots, d$.

Uma Teoria Quântica de Campos (Euclidiana) na Rede (ou para abreviar, TQC na rede) está determinada por um estado $\mu \in S_{C\left(S^{\mathbb{K}^{d+1}}\right)}$ (ou seja, um funcional linear, positivo e normalizado em $\left.C\left(S^{\mathbb{Z}^{d+1}}\right)\right)$. Supõe-se que $\mu$ satisfaz determinadas propriedades, entre as quais:

AR1 Invariância:

$$
\mu \circ \theta_{i, a}=\mu=\mu \circ \tau_{x} \forall i, a, \forall x .
$$

AR2 Positividade por Reflexões:

$$
\mu\left(\overline{\theta_{i, a} f} f\right) \geqslant 0 \quad \forall f \in \mathcal{P}\left(\Lambda_{i, a}\right), \forall i, a .
$$

(Aqui $\Lambda_{i, a}$ é o semi-espaço $\left\{x \in \mathbb{Z}^{d+1}: x_{i} \geqslant a\right\}$ ). 
Seja $\mathcal{H}_{0}:=\mathcal{P}\left(\Lambda_{0,0}\right)$. É imediato verificar que $\langle f, g\rangle_{\mathcal{H}_{0}}:=\mu(\overline{\theta f} g)$ é uma forma sesquilinear, hermitiana, não negativa (por AR2) em $\mathcal{H}_{0}$. Se $\mathcal{I}:=\left\{f \in \mathcal{H}_{0}\right.$ : $\left.\langle f, f\rangle_{\mathcal{H}_{0}}=0\right\}$, então completando o quociente $\mathcal{H}_{0} / \mathcal{I}$ obtem-se um espaço de Hilbert cujos vetores são classes de equivalência $[F]$ de seqüências de Cauchy $F$ de classes de equivalência $[f]$ de funções $f$ em $\mathcal{P}\left(\Lambda_{0,0}\right)$, tendo-se:

- $\|f\|_{\mathcal{H}_{0}}=\mu(\overline{\theta f} f)^{1 / 2}$,

- $\|[f]\|_{\mathcal{H}_{0} / \mathcal{I}}=\|f\|_{\mathcal{H}_{0}}$,

- $\|F\|=\lim _{n \rightarrow \infty}\left\|[f]_{n}\right\|_{\mathcal{H}_{0} / \mathcal{I}}$ se $F=\left\{[f]_{n}\right\}_{n \in \mathbb{N}}$,

- $\|[F]\|_{\mathcal{H}}=\|F\|$.

Seja $i: \mathcal{H}_{0} \longmapsto \mathcal{H}$ dada por $i(f):=\left[F_{f}\right]$ onde $F_{f}=\{[f],[f], \ldots\}$. Observe que:

$$
\left\|\left[F_{f}\right]\right\|_{\mathcal{H}}=\left\|F_{f}\right\|=\lim \|[f]\|_{\mathcal{H}_{0} / \mathcal{I}}=\|[f]\|_{\mathcal{H}_{0} / \mathcal{I}}=\|f\|_{\mathcal{H}_{0}} .
$$

Assim, $i$ é uma isometria linear bem definida. Além disso, tem imagem densa em $\mathcal{H}$.

2.1.3 Observações: Se definimos $\langle f, f\rangle_{2}:=\mu(\bar{f} f)=:\|f\|_{2}^{2}$ que também é uma forma sesquilinear hermitiana e não negativa (pela suposta positividade de $\mu$ ) e satisfaz portanto a desigualdade de Schwarz, temos:

(a) Pela invariância AR1, $\theta_{i, a}$ e $\tau_{x}$ são isometrias na norma $\|\cdot\|_{2}$.

(b) $\|f\|_{\mathcal{H}_{0}} \leqslant\|f\|_{2} \leqslant\|f\|_{\infty}$.

(c) Se um operador auto-adjunto $A$ em $\mathcal{H}_{0}$ é isométrico para a norma $\|\cdot\|_{2}$ e satisfaz:

$$
\|A f\|_{\mathcal{H}_{0}}^{2} \leqslant\|f\|_{\mathcal{H}_{0}}\left\|A^{2} f\right\|_{\mathcal{H}_{0}} \forall f \in \mathcal{H}_{0},
$$

então é limitado com $\|A\|_{B\left(\mathcal{H}_{0}\right)} \leqslant 1$. Com efeito, aplicando reiteradas vezes a desigualdade anterior conjuntamente com a desigualdade de Schwarz e o ponto (b) anterior temos:

$$
\begin{gathered}
\|A f\|_{\mathcal{H}_{0}} \leqslant\|f\|_{\mathcal{H}_{0}}^{\frac{1}{2}}\left\|A^{2} f\right\|_{\mathcal{H}_{0}}^{\frac{1}{2}} \leqslant\|f\|_{\mathcal{H}_{0}}^{\frac{1}{2}+\frac{1}{2^{2}}}\left\|A^{2^{2}} f\right\|_{\mathcal{H}_{0}}^{\frac{1}{2^{2}}} \\
\leqslant \cdots \leqslant\|f\|_{\mathcal{H}_{0}}^{\frac{1}{2}+\cdots+\frac{1}{2^{n}}}\left\|A^{2^{n}} f\right\|_{\mathcal{H}_{0}}^{\frac{1}{2^{n}}} \leqslant\|f\|_{\mathcal{H}_{0}}^{\frac{1}{2}+\cdots+\frac{1}{2^{n}}}\left\|A^{2^{n}} f\right\|_{2}^{\frac{1}{2^{n}}} \\
=\|f\|_{\mathcal{H}_{0}}^{\frac{1}{2}+\cdots+\frac{1}{2^{n}}}\|f\|_{2}^{\frac{1}{2^{n}}} \underset{n \rightarrow \infty}{\longrightarrow}\|f\|_{\mathcal{H}_{0}} .
\end{gathered}
$$


Todo operador limitado $A$ agindo em $\mathcal{H}_{0}$ que deixa $\mathcal{I}$ invariante induz um operador $i(A)$ em $\mathcal{H}$ densamente (e bem) definido por $i(A) i(f):=i(A f), \operatorname{com}\|i(A) i(f)\|_{\mathcal{H}}=$ $\|A f\|_{\mathcal{H}_{0}}$ e $i(A) i(B)=i(A B), i\left(A^{*}\right)=i(A)^{*}$. Isto acontece, em particular, para os $\tau_{x}$. Por exemplo, no caso dos $\tau_{n}$, usando 2.1.2(d) e AR1 no caso $n=0$ temos:

$$
\left\|\tau_{0} f\right\|_{\mathcal{H}_{0}}^{2}=\mu\left(\overline{\theta \tau_{0} f} \tau_{0} f\right)=\mu\left(\overline{\theta f} \tau_{0}^{2} f\right)=\left\langle f, \tau_{0}^{2} f\right\rangle \leqslant\|f\|_{\mathcal{H}_{0}}\left\|\tau_{0}^{2} f\right\|_{\mathcal{H}_{0}} .
$$

O operador resultante $T:=i\left(\tau_{0}\right)$ é chamado de matriz de transferência e é simples verificar que é auto-adjunto e, pela desigualdade acima, 2.1.3(a, b) e o fato que $\tau_{0}(\mathbb{I})=\mathbb{I}$, tem norma um. No caso $n=1, \ldots, d$ é igualmente simples verificar analogamente que os operadores resultantes $T_{n}:=i\left(\tau_{n}\right)$ são unitários (por 2.1.2(e)) e, como são mutuamente comutativos, existem operadores auto-adjuntos $P_{i}$ em $\mathcal{H}$ $\operatorname{com} \sigma\left(P_{i}\right) \subseteq(-\pi, \pi]$ tais que $\prod_{i=1}^{d} T_{i}^{j_{i}-k_{i}}=\mathrm{e}^{i(\mathbf{j}-\mathbf{k}) \cdot \mathbf{P}}$, onde $\mathbf{P}:=\left(P_{1}, \ldots, P_{d}\right)$. Tal $\mathbf{P}$ identifica-se com o operador de momento. O Hamiltoniano pode ser definido como $H=-\ln T$ desde que $\operatorname{ker} T=\{0\}$, o que acontece em casos típicos. ${ }^{5}$ No caso geral também denotaremos $T_{x}:=i\left(\tau_{x}\right)$.

Cada função $g \in \mathcal{H}_{0}$ induz um operador $A_{g}$ em $\mathcal{H}_{0}$ via multiplicação $A_{g} f(\sigma):=$ $g(\sigma) f(\sigma)$ com $\left\|A_{g}\right\|_{B\left(\mathcal{H}_{0}\right)} \leqslant\|g\|_{\infty}$, que é auto-adjunto se $\overline{\theta g}=g$. No caso particular de $g=p_{x}$ resulta $\left\|A_{p_{x}}\right\|_{B\left(\mathcal{H}_{0}\right)} \leqslant \sup \{|s|: s \in S\}$ e é auto-adjunto se $x=\left(0, x_{1}, \ldots, x_{d}\right) \in \mathbb{Z}^{d+1}$ e $S \subset \mathbb{R}$. No caso particular de $S=\{1,-1\}$ tem-se que $A_{p_{x}}^{2}=I_{\mathcal{H}_{0}}$ e $\left\|A_{p_{x}}\right\|_{B\left(\mathcal{H}_{0}\right)}=1$. Os operadores induzidos em $\mathcal{H}$ correspondentes serão denotados $P_{x}:=i\left(A_{p_{x}}\right)$. Observe-se que $T_{x_{1}} P_{x_{2}}=P_{x_{2}+x_{1}} T_{x_{1}}$.

Definem-se as funções de $\mathrm{n}$ pontos ou funções de Schwinger na rede como sendo:

$$
\mathcal{S}_{n}\left(x_{1}, \ldots, x_{n}\right):=\mu\left(p_{x_{1}} \cdots p_{x_{n}}\right) .
$$

Denotando $\Omega:=i(\mathbb{I}) \in \mathcal{H}$ e usando as definições e propriedades anteriores tem-se:

$$
\begin{aligned}
& \mathcal{S}_{n}\left(x_{1}, \ldots, x_{n}\right)=\mu\left(p_{0} p_{x_{2}-x_{1}} \cdots p_{x_{n}-x_{1}}\right) \\
& =\left\langle P_{0} \Omega, P_{x_{2}-x_{1}} P_{x_{3}-x_{1}} \cdots P_{x_{n-1}-x_{1}} P_{x_{n}-x_{1}} \Omega\right\rangle \\
& =\left\langle P_{0} \Omega, P_{x_{2}-x_{1}} P_{x_{3}-x_{1}} \cdots P_{x_{n-1}-x_{1}} P_{x_{n}-x_{1}} T_{x_{n}-x_{1}} \Omega\right\rangle \\
& =\left\langle P_{0} \Omega, P_{x_{2}-x_{1}} P_{x_{3}-x_{1}} \cdots P_{x_{n-1}-x_{1}} T_{x_{n}-x_{1}} P_{0} \Omega\right\rangle \\
& =\left\langle P_{0} \Omega, P_{x_{2}-x_{1}} P_{x_{3}-x_{1}} \cdots P_{x_{n-1}-x_{1}} T_{x_{n-1}-x_{1}} T_{x_{n-x_{n-1}}} P_{0} \Omega\right\rangle \\
& =\left\langle P_{0} \Omega, P_{x_{2}-x_{1}} P_{x_{3}-x_{1}} \cdots T_{x_{n-1}-x_{1}} P_{0} T_{x_{n}-x_{n-1}} P_{0} \Omega\right\rangle=\cdots \\
& \cdots=\left\langle P_{0} \Omega, T_{x_{2}-x_{1}} P_{0} T_{x_{3}-x_{2}} P_{0} \cdots T_{x_{n-x_{n-1}}} P_{0} \Omega\right\rangle .
\end{aligned}
$$

${ }^{5}[62],[30]$. 
Desta última identidade, usando que $T_{x}=T^{x_{0}} \prod_{i=1}^{d} T_{i}^{x_{i}}=\mathrm{e}^{-x_{0} H} \mathrm{e}^{i \text { x.P }}$ e que, pela invariância de $\mu$ por reflexões, $\mathcal{S}_{2}\left(\left(x_{0}, \mathrm{x}\right), 0\right)=\mathcal{S}_{2}\left(\left(\left|x_{0}\right|, \mathrm{x}\right), 0\right)$, segue como caso particular a assim chamada fórmula de Gellmann-Low: ${ }^{6}$

$$
S_{2}\left(x_{0}, \mathrm{x}\right):=\mathcal{S}_{2}\left(\left(x_{0}, \mathrm{x}\right), 0\right)=\left\langle\Omega, P_{0} \mathrm{e}^{-\left|x_{0}\right| H} \mathrm{e}^{i \mathrm{x} . \mathrm{P}} P_{0} \Omega\right\rangle .
$$

Denotando:

$$
\begin{aligned}
& T^{d}:=(-\pi, \pi]^{d} \\
& E_{\lambda}:=\text { família espectral de }(H, \mathbf{P})=: E_{\left(\lambda_{0}, \lambda\right)}
\end{aligned}
$$

e $d \mu\left(\lambda_{0}, \boldsymbol{\lambda}\right):=d\left\langle P_{0} \Omega, E_{\lambda} P_{0} \Omega\right\rangle$, da fórmula de Gellmann-Low temos:

$$
S_{2}\left(x_{0}, \mathrm{x}\right)=\int_{[0, \infty)} \int_{T^{d}} \mathrm{e}^{-\left|x_{0}\right| \lambda_{0}} \mathrm{e}^{i \mathrm{x} \cdot \boldsymbol{\lambda}} d \mu\left(\lambda_{0}, \lambda\right) .
$$

Esta expressão constitui um ponto de partida para o estudo da função de 2 pontos assim como das propriedades espectrais do operador de energia-momento (Veja-se 5.1.4(d) p. 90). É oportuno observar que, para evitar questões referentes à existência do Hamiltoniano $H$, a teoria pode ser desenvolvida igualmente em termos da matriz transferência $T$. Como isto não introduz mudanças significativas, preferimos adotar na presente a primeira abordagem, por uma mera questão de comodidade.

\subsection{O Campo Livre na Rede}

Para funções definidas na rede, a transformada de Fourier é na verdade uma série de Fourier:

$$
\begin{gathered}
\widehat{f}(p)=(2 \pi)^{-\frac{d+1}{2}} \sum_{x \in \mathbb{Z}^{d+1}} \mathrm{e}^{-i p \cdot x} f(x) \\
\check{f}(x)=(2 \pi)^{-\frac{d+1}{2}} \int_{T^{d+1}} \mathrm{e}^{i x . p} f(p) d p .
\end{gathered}
$$

Para funções $f$ na rede definem-se os operadores:

$$
\begin{aligned}
& \Delta_{i} f(x):=f\left(x+e_{i}\right)-f(x) \\
& \nabla_{i} f(x):=f(x)-f\left(x-e_{i}\right) .
\end{aligned}
$$

É simples verificar que $\Delta_{i}^{*}=-\nabla_{i}$. Define-se também o Laplaciano discreto:

$$
\Delta:=\sum_{i=0}^{d} \nabla_{i} \Delta_{i}=-\sum_{i=1}^{d} \Delta_{i}^{*} \Delta_{i} .
$$

${ }^{6}$ Cf. [84] pp. 215-216. 
A função de 2 pontos do campo livre é obtida resolvendo a equação:

$$
\left(-\Delta+m_{0}^{2}\right) S_{2}=\delta
$$

cuja transformada de Fourier é:

$$
\left[\sum_{i=0}^{d} 2\left(1-\cos \left(p_{i}\right)\right)+m_{0}^{2}\right] \widehat{S}_{2}(p)=(2 \pi)^{-\frac{d+1}{2}}
$$

ou seja:

$$
\widehat{S}_{2}(p)=\frac{(2 \pi)^{-\frac{d+1}{2}}}{\sum_{i=0}^{d} 2\left(1-\cos \left(p_{i}\right)\right)+m_{0}^{2}}=\frac{(2 \pi)^{-\frac{d+1}{2}}}{\sum_{i=0}^{d} 4 \operatorname{sen}^{2}\left(p_{i} / 2\right)+m_{0}^{2}} .
$$

Se para cada $i=0,1, \ldots, d$ denotamos $\widetilde{p}_{i}:=2 \operatorname{sen}\left(p_{i} / 2\right)$, temos que o denominador da função de 2 pontos é nulo se e somente se:

$$
\widetilde{p}_{0}= \pm i\left(|\widetilde{\mathrm{p}}|^{2}+m_{0}^{2}\right)^{1 / 2}
$$

ou equivalentemente:

$$
p_{0}= \pm i \omega_{0}(\mathrm{p})
$$

onde $\omega_{0}(\mathbf{p})$ é tal que $2 \operatorname{senh}\left(\omega_{0}(\mathbf{p}) / 2\right)=\left(|\widetilde{\mathbf{p}}|^{2}+m_{0}^{2}\right)^{1 / 2}$. Disto segue que:

$$
\begin{aligned}
\omega_{0}(\mathbf{p})=\operatorname{arcosh}\left[1+\frac{1}{2}\left(|\widetilde{\mathrm{p}}|^{2}+m_{0}^{2}\right)\right] \\
=2 \ln \left\{\left[1+\frac{1}{4}\left(|\widetilde{\mathrm{p}}|^{2}+m_{0}^{2}\right)\right]^{1 / 2}+\frac{1}{2}\left(|\widetilde{\mathrm{p}}|^{2}+m_{0}^{2}\right)^{1 / 2}\right\} .
\end{aligned}
$$

Esta última expressão é a relação de dispersão para o caso do campo livre. Para p pequeno, aproximando $\omega_{0}(\mathbf{p})$ por seu desenvolvimento em série de Taylor na origem até segunda ordem tem-se: ${ }^{7}$

onde:

$$
\omega_{0}(\mathbf{p}) \approx m_{1}+\frac{1}{2 m_{2}} \mathbf{p}^{2}
$$

$$
\begin{aligned}
& m_{1}=\omega_{0}(0)=2 \ln \left[\left(1+\frac{1}{4} m_{0}^{2}\right)^{1 / 2}+\frac{1}{2} m_{0}\right] \\
& m_{2}=\frac{1}{\omega_{0}^{\prime \prime}(0)}=\operatorname{senh}\left(m_{1}\right)=m_{0}\left(1+\frac{m_{0}^{2}}{4}\right)^{1 / 2} .
\end{aligned}
$$

\footnotetext{
${ }^{7}[65]$ p. 63.
} 


\subsection{Teoria do Espalhamento na Rede}

A teoria do espalhamento na rede foi originalmente desenvolvida por J. C. A. Barata e K. Fredenhagen. ${ }^{8}$ Nesta seção estão resumidos os principais resultados de [4].

Se partindo da expressão (2.1.1) p. 21, mas denotando agora $T_{x}=\mathrm{e}^{-t H} U(\mathrm{x})$ onde $U(\mathrm{x}):=\mathrm{e}^{i \mathrm{x} . \mathrm{P}}$ para cada $(t, \mathrm{x}) \in \mathbb{R} \times \mathbb{Z}^{d}$, formalmente trocamos $t$ por $i t$ obtemos as funções de Wightman na rede:

$$
\begin{aligned}
& \mathcal{W}_{n}\left(\left(t_{1}, x_{1}\right), \ldots,\left(t_{n}, x_{n}\right)\right) \\
& :=\left\langle\Omega, P_{0} \mathrm{e}^{-i H\left(t_{2}-t_{1}\right)} U\left(\mathrm{x}_{2}-\mathrm{x}_{1}\right) P_{0} \mathrm{e}^{-i H\left(t_{3}-t_{2}\right)} U\left(\mathrm{x}_{3}-\mathrm{x}_{2}\right) P_{0} \cdots\right. \\
& \left.\cdots \mathrm{e}^{-i H\left(t_{n}-t_{n-1}\right)} U\left(\mathrm{x}_{n}-\mathrm{x}_{n-1}\right) P_{0} \Omega\right\rangle .
\end{aligned}
$$

Para cada $h \in C_{0}^{\infty}\left(\mathbb{R}^{d+1}\right)$ definimos:

$$
\left.\phi(h):=\sum_{\mathbf{x} \in \mathbb{Z}^{d}} \int d t h(t, \mathbf{x}) \mathrm{e}^{-i t H} U(\mathbf{x}) P_{0} U(-\mathbf{x})\right) \mathrm{e}^{i t H} .
$$

2.3.1 Observação: Segue desta definição que as $\mathcal{W}_{n}$ consideradas como distribuições regulares satisfazem:

$$
\mathcal{W}_{n}\left(h_{1} \otimes \cdots \otimes h_{n}\right)=\left\langle\Omega, \phi\left(h_{1}\right) \cdots \phi\left(h_{n}\right) \Omega\right\rangle .
$$

Também é simples verificar que:

(a) $\phi\left(h \circ \tau_{(t, \mathrm{x})}\right)=\mathrm{e}^{i t H} U(-\mathrm{x}) \phi(h) U(\mathrm{x}) \mathrm{e}^{-i t H}$

(b) $\phi(h)^{*}=\phi(\bar{h})$

(c) $\|\phi(h)\| \leqslant$ const $|\widehat{h}(0)|$

(d) $\phi(h) \Omega=\int \widehat{h}(t, \mathrm{x}) d E_{(t, \mathrm{x})} P_{0} \Omega=\widehat{h}(H, \mathbf{P}) P_{0} \Omega$.

Introduzimos agora a seguinte hipótese:

AR3 Fatorização Assintótica (ou Propriedade de Cluster) Exponencial da Função de 2 pontos:

Existe uma constante $m>0$ tal que:

$$
\left|\mu\left(p_{x_{1}} p_{x_{2}}\right)-\mu\left(p_{x_{1}}\right) \mu\left(p_{x_{2}}\right)\right| \leqslant \text { const } \mathrm{e}^{-m\left|x_{1}-x_{2}\right|} \forall x_{1}, x_{2} .
$$

Esta suposição adicional permite provar o seguinte resultado, análogo na rede para a propriedade de cluster das distribuições de Wightman truncadas no contínuo: ${ }^{9}$

${ }^{8}[1],[2],[3],[4]$.

${ }^{9}$ Cf. $[78]$ p. 324 . 
2.3.2 Teorema: Para cada $\epsilon \in(0,1)$ e para cada $m \in \mathbb{N}$ existe uma constante positiva $c_{m, \epsilon}=c_{m, \epsilon}\left(h_{i}\right)$ tal que:

$$
\left|\mathcal{W}_{n}^{T}\left(\left(h_{1} \circ \tau_{x_{1}}\right) \otimes \cdots \otimes\left(h_{n} \circ \tau_{x_{n}}\right)\right)\right| \leqslant c_{m, \epsilon} \frac{(1+\|t\|)^{m}}{(1+\|\mathrm{x}\|)^{(m-1)-\epsilon}}
$$

onde: $\|t\|:=\max _{1 \leqslant i \leqslant n-1}\left|t_{i+1}-t_{i}\right| e\|\mathrm{x}\|:=\max _{i, j \in\{1, \ldots, n\}}\left|\mathrm{x}_{i}-\mathrm{x}_{j}\right|$.

Esquema da Prova: Usando o fato que a fórmula de Cartier ${ }^{10}$ permite expressar as funções truncadas como correlações ordinárias em uma teoria "produto tensorial", a prova pode ser reduzida ao caso das $\mathcal{W}_{n}$ (sem truncar). O próximo passo é expressar $\mathcal{W}_{n}$ en função das correlações euclidianas para assim poder aplicar a propriedade AR3. Isto é feito pelo uso da transformada de Chevishev como segue. Toda função suficientemente regular $f$ pode ser expressada como:

$$
f(H)=\sum_{n=0}^{\infty} b_{n}(f) T_{2 n}\left(\mathrm{e}^{-H / 2}\right),
$$

onde os $T_{n}(x)=\cos (n \arccos x)$ são os polinômios de Chebishev, os quais constituem uma base ortogonal de $L^{2}\left([-1,1],\left(1-x^{2}\right)^{-1 / 2} d x\right)$ e:

$$
b_{n}(f)=\frac{2}{\pi}\left(2-\delta_{n, 0}\right) \int_{0}^{\pi / 2} f(-2 \ln \cos \alpha) \cos (2 n \alpha) d \alpha .
$$

Para $f \in C_{0}^{\infty}$ os coeficientes $b_{n}(f)$ são rapidamente decrescentes com $n$. Assim, em particular:

$$
\begin{aligned}
\mathrm{e}^{-i t H} & =\sum_{k=0}^{\infty} b_{k}(t) T_{2 k}\left(\mathrm{e}^{-H / 2}\right), \\
b_{k}(t) & =\frac{2}{\pi}\left(2-\delta_{k, 0}\right) \int_{0}^{\pi / 2} \mathrm{e}^{-2 i t \ln \cos \alpha} \cos (2 k \alpha) d \alpha .
\end{aligned}
$$

Portanto:

$$
\begin{aligned}
& \mathcal{W}_{n}\left(\left(h_{1} \circ \tau_{x_{1}}\right) \otimes \cdots \otimes\left(h_{n} \circ \tau_{x_{n}}\right)\right)=\left\langle\Omega, \phi\left(h_{1} \circ \tau_{x_{1}}\right) \cdots \phi\left(h_{n} \circ \tau_{x_{n}}\right) \Omega\right\rangle \\
& =\left\langle\Omega, \mathrm{e}^{-i H t_{1}} U\left(\mathrm{x}_{1}\right) \phi\left(h_{1}\right) U\left(-\mathrm{x}_{1}\right) \mathrm{e}^{i H t_{1}} \cdots \mathrm{e}^{-i H t_{n}} U\left(\mathrm{x}_{n}\right) \phi\left(h_{n}\right) U\left(-\mathrm{x}_{n}\right) \mathrm{e}^{i H t_{n}} \Omega\right\rangle \\
& =\left\langle\Omega, \phi\left(h_{1}\right) \mathrm{e}^{-i H\left(t_{2}-t_{1}\right)} U\left(\mathrm{x}_{2}-\mathrm{x}_{1}\right) \phi\left(h_{2}\right) \cdots \mathrm{e}^{-i H\left(t_{n}-t_{n-1}\right)} U\left(\mathrm{x}_{n}-\mathrm{x}_{n-1}\right) \phi\left(h_{n}\right) \Omega\right\rangle \\
& =\sum_{k_{1}, \ldots, k_{n-1}=0}^{\infty} b_{k_{1}}\left(t_{2}-t_{1}\right) \cdots b_{k_{n-1}}\left(t_{n}-t_{n-1}\right) c_{k_{1}, \ldots, k_{n-1}}\left(\mathrm{x}_{1}, \ldots, \mathrm{x}_{n}\right),
\end{aligned}
$$

\footnotetext{
${ }^{10}[1]$ apêndice B, p. 85 . Cf. [78] p. 381 .
} 
onde:

$$
\begin{aligned}
& c_{k_{1}, \ldots, k_{n-1}}\left(\mathrm{x}_{1}, \ldots, \mathrm{x}_{n}\right) \\
& =\left\langle\Omega, \phi\left(h_{1}\right) T_{2 k_{1}}\left(\mathrm{e}^{-H / 2}\right) U\left(\mathrm{x}_{2}-\mathrm{x}_{1}\right) \phi\left(h_{2}\right) \cdots T_{2 k_{n-1}}\left(\mathrm{e}^{-H / 2}\right) U\left(\mathrm{x}_{n}-\mathrm{x}_{n-1}\right) \phi\left(h_{n}\right) \Omega\right\rangle .
\end{aligned}
$$

Na seqüência, a prova baseia-se nas propriedades de decaimento dos coeficientes $b_{k_{i}}$ e $c_{k_{1}, \ldots, k_{n-1}}$, a saber:

$$
\begin{aligned}
\left|\prod_{i=1}^{n-1} b_{k_{i}}\left(t_{i+1}-t_{i}\right)\right| & \leqslant c_{M}\left(\frac{1+\|t\|}{1+|k|}\right)^{M} \quad \forall M \geqslant 0 \\
\left|c_{k_{1}, \ldots ., k_{n-1}}\left(\mathrm{x}_{1}, \ldots, \mathrm{x}_{n}\right)\right| & \leqslant \text { const } \\
\left|c_{k_{1}, \ldots, k_{n-1}}\left(\mathrm{x}_{1}, \ldots, \mathrm{x}_{n}\right)\right| & \leqslant \text { const } \mathrm{e}^{A(m)|k|-m\|x\|}
\end{aligned}
$$

onde $|k|=\sum_{i=1}^{n-1} k_{i}$ e $A(m)=2 \operatorname{arcsenh}\left(\mathrm{e}^{-m / 2}\right)$. A primeira desigualdade segue das propriedades dos $b_{k}$ a partir da sua definição (basicamente, integrando por partes), a segunda da limitação dos $T_{2 k}, \phi(h)$ e $U(\mathrm{x})$ e a terceira de AR3. Para os detalhes veja-se [4] p. 514, ou [1] cap. 2.

A hipótese AR3 tem como conseqüência adicional a unicidade do vácuo e o gap de massa inferior no espectro do Hamiltoniano. ${ }^{11}$ Em determinados modelos, ela segue da convergência de certas expansões em polímeros. ${ }^{12}$ Para o caso do modelo de Ising na região de altas e baixas temperaturas, vejam-se [84], [85], [69], [70]. Expansões em polímeros serão introduzidas na seção 4.3.

Corresponde agora a construção dos estados assintóticos. Para isto é necessário introduzir a seguinte suposição:

AR4 Existência de Estados de Uma Partícula:

A transformada de Fourier da função de 2 pontos $S_{2}\left(p_{0}, \mathbf{p}\right)$ pode ser estendida analiticamente à região $\left\{p_{0}: \operatorname{Im} p_{0}<\widetilde{\omega}(\mathrm{p})\right\}$ com um polo simples em $p_{0}=i \omega(\mathrm{p})$. Supõe-se que a relação de dispersão da partícula $\omega(\mathrm{p})$ é real analítica, $\widetilde{\omega}(\mathbf{p})$ é contínua, $\widetilde{\omega}(\mathbf{p})>\omega(\mathbf{p}) \geqslant m \mathrm{e} \operatorname{det}\left(\partial^{2} \omega(\mathbf{p}) / \partial p_{i} \partial p_{j}\right) \neq 0 \forall \mathbf{p}$.

A suposição AR4 foi verificada para diversos modelos na rede em uma série de trabalhos de R. Schor, M. O'Carroll e outros, [85], [84], [69], [70]. Esta suposição implica a existência de um subespaço fechado $\mathcal{H}_{1} \subseteq \mathcal{H}$ no qual $H=\omega(\mathbf{P})$. $\mathcal{H}_{1}$ é a clausura do subespaço $D_{1}$ dado por:

$$
\begin{aligned}
D_{1}=\left\{\phi(h) \Omega: \widehat{h} \in \mathcal{D}\left(\mathbb{R}^{d+1}\right)\right. & \\
& \left.\quad \mathrm{supp}\{\widehat{h}\} \cap \sigma(H, \mathbf{P}) \subseteq\left\{(\omega(\mathrm{p}), \mathrm{p}): \mathrm{p} \in(-\pi, \pi]^{d}\right\}\right\} .
\end{aligned}
$$

${ }^{11}$ Veja-se 5.1.4(a, b) na p. 90.

${ }^{12}[37]$. Cf. [93]. 
Seja $f$ uma solução da equação de onda correspondente à relação de dispersão $\omega$, ou seja:

$$
f(t, \mathrm{x})=\frac{1}{(2 \pi)^{d / 2}} \int_{(-\pi, \pi]^{d}} \mathrm{e}^{i(\mathrm{x} \cdot \mathrm{p}-\omega(\mathrm{p}) t)} g(\mathrm{p}) d \mathrm{p},
$$

com (a transformada de Fourier do) "dado inicial" $g \in C^{\infty}\left((-\pi, \pi]^{d}\right)$. Definimos:

$$
\begin{aligned}
B_{\phi, h}(t, \mathrm{x}) & :=\phi\left(h \circ \tau_{x}\right) \\
B_{\phi, h}^{f}(t) & :=\sum_{\mathbf{x} \in \mathbb{Z}^{d}} f(t, \mathrm{x}) B_{\phi, h}(t, \mathrm{x}) .
\end{aligned}
$$

Analogamente ao caso no contínuo, os vetores $B_{\phi, h}(t, \mathrm{x}) \Omega \operatorname{com} \phi(h) \Omega \in D_{1}$ são solução do problema de 1 corpo e também neste caso $B_{\phi, h}^{f}(t) \Omega$ independe de $t$.

Se $f$ é como (2.3.1) com dado inicial $g$ denotamos $\mathcal{G}^{f}$ uma vizinhança de $\{\operatorname{grad} \omega(\mathbf{p})$ : $\mathrm{p} \in \operatorname{supp} g\}$.

2.3.3 Proposição: Se $f$ é como em (2.3.1), então:

(a) Para cada $m \in \mathbb{N}$ existe $c_{m}$ tal que:

$$
|f(t, \mathrm{x})| \leqslant \frac{c_{m}}{(1+|\mathrm{x}|+|t|)^{m}} \quad \text { se }|\mathrm{x}| \notin t \mathcal{G}^{f}
$$

(b)

$$
\sum_{\mathbf{x} \in \mathbb{Z}^{d}}|f(t, \mathrm{x})| \leqslant c(1+|t|)^{d / 2}
$$

Referências: Veja-se [78] p. 38 e p. 44 respectivamente.

Seja $n \in \mathbb{N}$ arbitrário. Diremos que um conjunto $\left\{f_{i}\right\}_{i=1}^{n}$ de soluções como (2.3.1) é disjunto se para cada $i=1, \ldots, n$ tem-se que $\mathcal{G}^{u} \cap \mathcal{G}^{v}=\emptyset$ cada vez que $u \notin\left\{f_{i}, \partial_{t} f_{i}, \overline{f_{i}}, \overline{\partial_{t} f_{i}}\right\} \ni v$. Para cada $i=1, \ldots, n$ sejam $\phi_{i}:=\phi\left(h_{i}\right) \Omega \in D_{1}$. Definimos:

$$
\begin{aligned}
\mathcal{P}_{h} & :=\left\{\mathrm{p} \in(-\pi, \pi]^{d}:(\omega(\mathrm{p}), \mathrm{p}) \in \operatorname{supp}\{\widehat{h}\} \cap \sigma(H, \mathbf{P})\right\} \\
\mathcal{G}_{i} & :=\left\{\operatorname{grad} \omega(\mathrm{p}): \mathrm{p} \in \mathcal{P}_{h_{i}}\right\}
\end{aligned}
$$

e dizemos que o conjunto de vetores $\left\{\phi_{i}:=\phi\left(h_{i}\right) \Omega\right\}_{i=1}^{n} \subset D_{1}$ é disjunto se $\mathcal{G}_{i} \cap \mathcal{G}_{j}=$ $\emptyset$ se $i \neq j$. Finalmente definimos:

$$
\eta(t):=B_{\phi, h_{1}}^{f_{1}}(t) \cdots B_{\phi, h_{n}}^{f_{n}}(t) \Omega .
$$

Nestas circunstâncias temos o seguinte 
2.3.4 Teorema: Seja $\left\{f_{i}\right\}_{i=1}^{n}$ um conjunto disjunto de soluções como em (2.3.1). Então:

(a) Os limites

$$
\lim _{t \rightarrow \mp \infty} \eta(t)=: \eta_{\text {out }}^{\text {in }}\left(\phi_{1}, \ldots, \phi_{n}\right)
$$

existem em $\mathcal{H}$ dependendo apenas dos $\phi_{i} \in D_{1}$ e para cada $m \in \mathbb{N}$ tem-se:

$$
\left\|\eta(t)-\eta_{\text {out }}\left(\phi_{1}, \ldots, \phi_{n}\right)\right\| t^{m} \underset{t \rightarrow \mp \infty}{\longrightarrow} 0 .
$$

(b) Suponha-se adicionalmente que o conjunto disjunto de soluções $\left\{f_{i}\right\}_{i=1}^{n}$ possui dados iniciais $\left\{g_{i}\right\}_{i=1}^{n}$ respectivamente com $g_{i} \equiv 1$ em $\mathcal{P}_{h_{i}}$ para cada $i=$ $1, \ldots, n$ e que $\left\{\phi_{i}:=\phi\left(h_{i}\right) \Omega\right\}_{i=1}^{n}$ é um conjunto disjunto de vetores em $D_{1}$. Então, se $\left\{\psi_{i}\right\}_{i=1}^{k}$ é um outro conjunto disjunto de vetores em $D_{1}$ tem-se:

$$
\left\langle\eta_{\text {out }}^{\text {in }}\left(\psi_{1}, \ldots, \psi_{k}\right), \eta_{\text {out }}^{\text {in }}\left(\phi_{1}, \ldots, \phi_{n}\right)\right\rangle=\delta_{n k} \sum_{\sigma \in S(n)} \prod_{i=1}^{n}\left\langle\psi_{i}, \phi_{\sigma(i)}\right\rangle
$$

onde $S(n)$ denota o grupo de permutações de ordem $n$.

Referências: Veja-se [4] pp. 516-517. A prova da parte (a) é totalmente análoga à do caso no contínuo. ${ }^{13}$ A prova da parte (b) também é simples. Digamos apenas a título de observação que a condição sobre as $g_{i}$ implica que $B_{\phi, h_{i}}^{f_{i}}(t) \Omega=\phi_{i}$. No entanto, a condição dos vetores $\left\{\phi_{i}\right\}_{i=1}^{n}$ serem disjuntos é suficiente para ter funções $\left\{g_{i}\right\}_{i=1}^{n} \operatorname{com} g_{i} \equiv 1$ em $\mathcal{P}_{h_{i}}$ diferentes e assim poder gerar um conjunto disjunto de soluções $\left\{f_{i}\right\}_{i=1}^{n}$.

Definem-se:

$$
D_{\text {out }}^{\text {out }}:=\operatorname{span}\left\{\eta_{\text {out }}^{\text {in }}\left(\phi_{1}, \ldots, \phi_{n}\right):\left\{\phi_{i}\right\}_{i=1}^{n} \subset D_{1} \text { é disjunto }\right\}_{n \in \mathbb{N}} .
$$

Seja $\mathcal{F}\left(\mathcal{H}_{1}\right)$ o espaço de Fock sobre $\mathcal{H}_{1}$ e seja $\mathcal{F}_{0}$ o subespaço de $\mathcal{F}\left(\mathcal{H}_{1}\right)$ gerado pelos produtos tensoriais de elementos em $D_{1}$ com a propriedade de serem disjuntos. Definem-se $\underset{\text { out }}{U_{\text {in }}}: \mathcal{F}_{0} \longmapsto D_{\text {out }}^{\text {in }}$ por:

$$
U_{\text {in }}^{\text {out }}\left(\phi_{1} \otimes \cdots \otimes \phi_{n}\right):=\eta_{\substack{\text { in } \\ \text { out }}}\left(\phi_{1}, \ldots, \phi_{n}\right) .
$$

Pela propriedade 2.3.4(b) os operadores $U_{\text {in }}$ sã são isomêtricos, estendendo-se a $\overline{\mathcal{F}_{0}}$. Se $\operatorname{grad} \omega(\mathrm{p})$ é nunca constante como função de $\mathrm{p}$, então $\overline{\mathcal{F}_{0}}=\mathcal{F}\left(\mathcal{H}_{1}\right)$, podendo-se

\footnotetext{
${ }^{13} \mathrm{Cf}$. [47], [48].
} 
obter assim duas imagens isométricas $\mathcal{H}_{\text {in }}:=\overline{D_{\text {out }}}$ out do espaço de Fock $\mathcal{F}\left(\mathcal{H}_{1}\right)$ em $\mathcal{H}$. A interpretação física de $D_{\substack{\text { in } \\ \text { out }}}$ como subespaços de estados assintóticos pode ser verificada calculando os valores esperados de operadores representando detectores no sentido de Haag e Araki. ${ }^{14}$

${ }^{14}[1]$ sec. 3.2; Cf. [45]. Sobre "detectores", veja-se o comentário no final da seção 1.3, p. 13. 


\section{Capítulo 3}

\section{Completeza Assintótica}

Tudo o que está aqui está lá, o que não está aqui não está em lugar nenhum.

Vishvasâra Tantra ${ }^{1}$

\subsection{Introdução}

Em termos simples, ou "profanos", o contéudo da completeza assintótica é acertadamente expressado na epígrafe acima. Para formaliza-lo matematicamente basta observar que o "aqui" na segunda frase pode identificar-se com a totalidade dos estados fisicamente admissíveis, i.e., $\mathcal{H}$, e identificando o "lá" da primeira frase com os subespaços assintóticos $\mathcal{H}_{\text {in }} \subseteq \mathcal{H}$, esta última pode ser expressada como $\mathcal{H} \subseteq \mathcal{H}_{\text {out }}$. Ou seja, noutros termos:

$$
\mathcal{H}=\mathcal{H}_{\text {in }}^{\text {out }} \text {. }
$$

Como já foi expressado na seção 1.3, p. 10, numa teoria fisicamente razoável é de se esperar que valha a completeza assintótica, significando isto que cada estado de um sistema físico pode ser visto bem como descrevendo partículas (incluindo seus estados ligados) bem como decaindo num tal estado no transcurso do tempo (estando implícito nesta idéia que a dinâmica livre não tem estados ligados). Também, é uma questão matemática profunda e ainda em aberto estabelecer a completeza assintótica, ainda para os exemplos concretos existentes na teoria quântica de campos, onde existem resultados parciais na teoria $P(\varphi)_{2}$ fracamente acoplada na região de baixas energias.

\footnotetext{
${ }^{1}$ Citado em A. van Lysebeth, Tantra, o Culto da Feminilidade-Outra visão da vida e do sexo, Summus, São Paulo, 1994, p. 61.
} 
O estudo da completeza assintótica, como assim também do espectro correspondente a partículas e a estados ligados, usa a equação de Bethe-Salpeter. Este método é a contrapartida na Teoria Quântica de Campos dos métodos independentes do tempo na teoria do espalhamento na Mecânica Quântica. Formalmente, se $H$ e $H_{0}$ são operadores num espaço de Hilbert e $R$ e $R_{0}$ os seus respectivos resolventes, ${ }^{2}$ é trivial verificar a assim chamada segunda equação resolvente: ${ }^{3}$

$$
R-R_{0}=-R_{0}\left(H-H_{0}\right) R,
$$

de onde, chamando $K:=H-H_{0}$, tem-se:

$$
R=R_{0}-R_{0} K R \text {. }
$$

Em casos típicos é $H=H_{0}+V$, ou seja, $K=V$. Nesta analogia, o núcleo de Bethe-Salpeter $K$ corresponde ao potencial $V$. Na Teoria Quântica de Campos esta analogia ganha precisão no limite não relativista, $c \rightarrow \infty$, em cujo caso $K$ converge a $V$ após reescalamento apropriado. A equação de Bethe-Salpeter é um Ansatz inspirado na identidade (3.1.1), ou melhorando, no uso que dela é feito na procura de soluções perturbativas à equação de Lippmann-Schwinger na teoria do espalhamento na Mecânica Quântica. ${ }^{4}$ Com efeito, iterando-a repetidas vezes temse:

$$
\begin{aligned}
R & =R_{0}-R_{0} K R \\
& =R_{0}\left(I-K R_{0}\right)+\left(R_{0} K\right)^{2} R \\
& =R_{0}\left(I-K R_{0}+\left(K R_{0}\right)^{2}\right)-\left(R_{0} K\right)^{3} R \\
& =\cdots \\
& =R_{0}\left(\sum_{k=0}^{n}(-1)^{k}\left(K R_{0}\right)^{k}\right)+R_{n}
\end{aligned}
$$

onde $R_{n}=(-1)^{n+1}\left(R_{0} K\right)^{n+1} R$. Se $R_{n} \underset{n \rightarrow \infty}{\longrightarrow} 0$, isso conduz a uma expressão para $R$ como série de Neumann:

$$
R=R_{0} \sum_{k=0}^{\infty}(-1)^{k}\left(K R_{0}\right)^{k}=R_{0}\left(I+K R_{0}\right)^{-1},
$$

o que na verdade já é sugerido pela seguinte resolução "formal" da equação:

$$
\begin{aligned}
R=R_{0}-R_{0} K R \Longrightarrow & R_{0}^{-1} R=I-K R \Longrightarrow R_{0}^{-1} R=I-K R_{0} R_{0}^{-1} R \\
& \Longrightarrow\left(I+K R_{0}\right) R_{0}^{-1} R=I \Longrightarrow R=R_{0}\left(I+K R_{0}\right)^{-1}
\end{aligned}
$$

\footnotetext{
${ }^{2}$ Ou seja, $R=R(\lambda):=(H-\lambda I)^{-1}$ e analogamente para $R_{0}$ e $H_{0}$. Omitimos a menção a $\lambda$ para simplificar a notação.

${ }^{3}$ Veja-se [75] p. 478.

${ }^{4}$ Veja-se [75] pp. 472 e 479.
} 
Observe-se também que tomando inversos nesta última igualdade segue que:

$$
K=R^{-1}-R_{0}^{-1}
$$

a qual é uma expressão conveniente para $K$.

Na Teoria Quântica de Campos, $K$ é visto como um ente derivado, já que não aparece no operador de energia nem nas equações de movimento. Propriedades de analiticidade de $K$ no espaço de energia-momento, ${ }^{5}$ ou seja de $\widehat{K}$, podem ser usadas para estudar o espectro de massa e estados assintóticos de baixa energia. Este método se aplica ao problema de estados ligados e ressonâncias e completeza assintótica para baixas energias. As propriedades requeridas de analiticidade foram provadas para modelos $P(\varphi)_{2}$ na região de acoplamento fraco. ${ }^{6}$ Existem também trabalhos nos quais fizeram-se progressos no programa recíproco, a saber, postular propriedades para o espectro de massa e derivar propriedades de analiticidade de $\widehat{K} \cdot{ }^{7}$ A estratégia para provar a completeza assintótica na abordagem acima descrita segue as linhas diretrizes do trabalho de Spencer e Zirilli: ${ }^{8}$ da analiticidade de $K$ e da equação de Bethe-Salpeter segue que $R$ é basicamente uma perturbação analítica e compacta, em um espaço de Hilbert adequado, de $R_{0}$. Disso segue que $\left(I+K R_{0}\right)^{-1}$ existe salvo polos e $R=R_{0}\left(I+K R_{0}\right)^{-1}$, de onde segue, pela sua vez, que o espectro de $R$ é o mesmo que o espectro de $R_{0}$ salvo pela existência de tais polos. Introduzindo neste ponto uma hipótese extra para $K$, a saber, que na primeira ordem da teoria de perturbações está dado por uma função $\delta$ repulsiva, pode-se provar finalmente que não existem polos para energias suficientemente baixas. Isto determina a completeza assintótica "parcial", mencionada na seção 1.3, p. 12. A hipótese extra sobre a estrutura de $K$ é característica das interações $\lambda P(\varphi)_{2}$ e foi verificada em tais modelos para $\lambda$ pequeno, ou seja para acoplamento fraco. ${ }^{9}$ Métodos inspirados nesta técnica foram usados também no estudo de estados ligados ${ }^{10}$ em modelos de Landau-Ginzburg estocásticos na rede ${ }^{11}$ e sistemas de spin ferromagnéticos clássicos na região de altas temperaturas. ${ }^{12}$

Mas, antes de estabelecer a equação de Bethe-Salpeter na rede, devem ser introduzidos os análogos dos resolventes. Isto pode tornar-se plausível de maneira um tanto mais convincente, ou verossímil, no contexto mais geral da Teoria Quântica

\footnotetext{
${ }^{5}$ Equivalentemente, decaimento exponencial de $K$ nas variáveis do espaço-tempo.

${ }^{6}[99]$. Cf. [100].

${ }^{7}[10],[11],[12]$.

$8[101]$.

9 [99].

${ }^{10}[25],[26]$.

$11[90],[28]$.

${ }^{12}$ [88], [89].
} 
de Campos no contínuo. Sem entrar em detalhes, diremos apenas que o campo livre pode ser pensado como a TQC definida em termos das funções de Schwinger truncadas ${ }^{13}$ como:

$$
\mathcal{S}_{n}^{T}= \begin{cases}\mathcal{S}_{2}^{T} & \text { se } n=2 \\ 0 & \text { se } n \neq 2 .\end{cases}
$$

Em tal caso, tem-se que $\mathcal{S}_{2}=\mathcal{S}_{2}^{T}$, estando a função de 2 pontos determinada pela representação de Källen-Lehmann. ${ }^{14}$ Igualmente, as funções de $n$ pontos com $n$ ímpar são nulas e as com $n$ par são soma de produtos de funções de 2 pontos. ${ }^{15}$ Em uma tal teoria (com $\mathcal{S}_{2} \neq 0$ ) existem no espaço de Hilbert dos estados físicos $\mathcal{H}$ dois tipos de vetores não triviais mutuamente ortogonais, a saber, o vácuo $\psi_{0}$ e os "estados de uma partícula" $\phi(f) \psi_{0}$. Uma maneira de generalizar o campo livre seria introduzir em $\mathcal{H}$ mais vetores que sejam ortogonais aos já existentes. A escolha natural seriam os "estados de duas partículas" $\phi(f) \phi(g) \psi_{0}$. Supondo que nesta teoria mais geral as funções de $n$ pontos com $n$ ímpar também sejam nulas, a condição de ortogonalidade se reduz à condição de ortogonalidade apenas para com o vácuo. Uma condição suficiente para que os "estados de duas partículas" tenham projeção não nula no complemento ortogonal do vácuo é:

$0 \neq\left\|\phi(f) \phi(g) \psi_{0}-\left\langle\psi_{0}, \phi(f) \phi(g) \psi_{0}\right\rangle \psi_{0}\right\|^{2}=\mathcal{S}_{4}(\bar{g} \otimes \bar{f} \otimes f \otimes g)-\mathcal{S}_{2}(\bar{g} \otimes \bar{f}) \mathcal{S}_{2}(f \otimes g)$

(aqui usou-se a hermiticidade de $\mathcal{S}_{2}$ e $\left\|\psi_{0}\right\|=1$ ). Por sua vez, uma condição suficiente para isto último é:

$$
0 \neq \mathcal{S}_{4}(f \otimes g \otimes h \otimes k)-\mathcal{S}_{2}(f \otimes g) \mathcal{S}_{2}(h \otimes k)=: \mathcal{D}(f \otimes g \otimes h \otimes k) .
$$

Observe que $\mathcal{D}$ é a $\mathcal{S}_{4}^{T}$ "parcialmente somada". Definindo a parte restante como:

$$
\mathcal{D}_{0}(f \otimes g \otimes h \otimes k):=\mathcal{S}_{2}(f \otimes h) \mathcal{S}_{2}(g \otimes k)+\mathcal{S}_{2}(f \otimes k) \mathcal{S}_{2}(g \otimes h),
$$

tem-se que $\mathcal{S}_{4}^{T}=\mathcal{D}-\mathcal{D}_{0}$ (desde que as funções impares sejam nulas). Que $\mathcal{D}$ funciona como uma espécie de "resolvente parcial" será visto na seção 3.3, após introduzir as definições pertinentes na rede.

\subsection{O Núcleo de Bethe-Salpeter na Rede}

Definimos:

$$
\mathcal{D}\left(x_{1}, x_{2}, x_{3}, x_{4}\right):=\mathcal{S}_{4}\left(x_{1}, x_{2}, x_{3}, x_{4}\right)-\mathcal{S}_{2}\left(x_{1}, x_{2}\right) \mathcal{S}_{2}\left(x_{3}, x_{4}\right)
$$

${ }^{13}$ Veja-se, e.g., [78] p. 323.

${ }^{14}$ Veja-se [77] p. 70 ou [95] p. 74.

${ }^{15}$ Veja-se, e.g., [95] pp. 75-76. 


$$
\mathcal{D}_{0}\left(x_{1}, x_{2}, x_{3}, x_{4}\right):=\mathcal{S}_{2}\left(x_{1}, x_{3}\right) \mathcal{S}_{2}\left(x_{2}, x_{4}\right)+\mathcal{S}_{2}\left(x_{1}, x_{4}\right) \mathcal{S}_{2}\left(x_{2}, x_{3}\right),
$$

como sendo as partes conexa e não-conexa respectivamente da função de 4 pontos (truncada). Como já foi dito no final da seção anterior, numa TQC onde todas a funções de $n$ pontos são nulas quando $n$ é ímpar ${ }^{16}$ tem-se que $\mathcal{S}_{4}^{T}=\mathcal{D}-\mathcal{D}_{0}$. $\mathrm{O}$ núcleo de Bethe-Salpeter $\mathcal{N}$ está definido pela assim chamada equação de Bethe-Salpeter, a saber, $\mathcal{D}=\mathcal{D}_{0}-\mathcal{D}_{0} \mathcal{N} \mathcal{D}$, ou, em forma expandida:

$$
\begin{aligned}
\mathcal{D}\left(x_{1}, x_{2}, x_{3}, x_{4}\right) & \\
= & \mathcal{D}_{0}\left(x_{1}, x_{2}, x_{3}, x_{4}\right) \\
& -\sum_{y_{1}, y_{2}, y_{3}, y_{4} \in \mathbb{Z}^{d+1}} \mathcal{D}_{0}\left(x_{1}, x_{2}, y_{1}, y_{2}\right) \mathcal{N}\left(y_{1}, y_{2}, y_{3}, y_{4}\right) \mathcal{D}\left(y_{3}, y_{4}, x_{3}, x_{4}\right)
\end{aligned}
$$

Desde que considerado como núcleo "integral" o $\mathcal{N}$ possui várias simetrias, entre elas a invariância por translações, de onde existe $N$ tal que:

$$
\mathcal{N}\left(y_{1}, y_{2}, y_{3}, y_{4}\right)=N\left(y_{1}-y_{4}, y_{2}-y_{4}, y_{3}-y_{4}\right) \text {. }
$$

Com efeito, basta apenas definir $N\left(y_{1}, y_{2}, y_{3}\right):=\mathcal{N}\left(y_{1}, y_{2}, y_{3}, 0\right)$. Também, se para cada $n \in \mathbb{N}$ definimos:

$$
S_{n}\left(x_{1}, \ldots, x_{n-1}\right):=\mu\left(p_{0} p_{x_{1}} \ldots p_{x_{n-1}}\right),
$$

pela invariância translacional AR1, p. 19, das funções de $n$ pontos, temos:

$$
\mathcal{S}_{n}\left(x_{1}, \ldots, x_{n}\right)=S_{n}\left(x_{1}-x_{n}, \ldots, x_{n-1}-x_{n}\right) .
$$

Definimos agora um novo conjunto de variáveis por:

$$
\begin{array}{lll}
\xi:=x_{1}-x_{2} & u_{1}:=x_{1}+x_{2}-\left(y_{1}+y_{2}\right) \\
\eta:=x_{3}-x_{4} & u_{2}:=y_{1}-y_{2} \\
\tau:=x_{1}+x_{2}-\left(x_{3}+x_{4}\right) . & u_{3}:=y_{3}+y_{4}-\left(x_{3}+x_{4}\right) \\
& u_{4}:=y_{3}-y_{4} .
\end{array}
$$

Um cálculo direto prova que:

$$
\begin{aligned}
\mathcal{D}\left(x_{1}, x_{2}, x_{3}, x_{4}\right) & =S_{4}\left(x_{1}-x_{4}, x_{2}-x_{4}, x_{3}-x_{4}\right)-S_{2}\left(x_{1}-x_{2}\right) S_{2}\left(x_{3}-x_{4}\right) \\
& =S_{4}\left(\frac{\tau+\xi+\eta}{2}, \frac{\tau-\xi+\eta}{2}, \eta\right)-S_{2}(\xi) S_{2}(\eta)=: D(\tau, \xi, \eta) .
\end{aligned}
$$

\footnotetext{
${ }^{16}$ Por exemplo, no modelo de Ising. Veja-se 4.2 .3 p. 78.
} 
Analogamente:

$$
\begin{aligned}
& \mathcal{D}_{0}\left(x_{1}, x_{2}, x_{3}, x_{4}\right) \\
& =S_{2}\left(\frac{\tau+(\xi-\eta)}{2}\right) S_{2}\left(\frac{\tau-(\xi-\eta)}{2}\right)+S_{2}\left(\frac{\tau+(\xi+\eta)}{2}\right) S_{2}\left(\frac{\tau-(\xi+\eta)}{2}\right) \\
& =: D_{0}(\tau, \xi, \eta) \\
& \mathcal{D}_{0}\left(x_{1}, x_{2}, y_{1}, y_{2}\right) \\
& =S_{2}\left(\frac{u_{1}+\left(\xi-u_{2}\right)}{2}\right) S_{2}\left(\frac{u_{1}-\left(\xi-u_{2}\right)}{2}\right) \\
& +S_{2}\left(\frac{u_{1}+\left(\xi+u_{2}\right)}{2}\right) S_{2}\left(\frac{u_{1}-\left(\xi+u_{2}\right)}{2}\right)=D_{0}\left(u_{1}, \xi, u_{2}\right) \\
& \mathcal{D}\left(y_{3}, y_{4}, x_{3}, x_{4}\right)=S_{4}\left(\frac{u_{3}+u_{4}+\eta}{2}, \frac{u_{3}-u_{4}+\eta}{2}, \eta\right)-S_{2}\left(u_{4}\right) S_{2}(\eta) \\
& =D\left(u_{3}, u_{4}, \eta\right) \\
& \mathcal{N}\left(y_{1}, y_{2}, y_{3}, y_{4}\right)=N\left(y_{1}-y_{4}, y_{2}-y_{4}, y_{3}-y_{4}\right) \\
& =N\left(\frac{\left(\tau-u_{1}-u_{3}\right)+u_{2}+u_{4}}{2}, \frac{\left(\tau-u_{1}-u_{3}\right)-u_{2}+u_{4}}{2}, u_{4}\right) \\
& =: \check{K}\left(\tau-u_{1}-u_{3}, u_{2}, u_{4}\right) \text {. }
\end{aligned}
$$

3.2.1 Observação: No trabalho de Spencer e Zirilli, [101], p. 3, é utilizada uma mudança de variáveis, na qual nos baseamos para introduzir este novo conjunto de variáveis acima definidas, com a diferença de que omitimos o fator $1 / 2$ presente naquelas. Esta ausência é motivada no presente caso na necessidade de obter uma mudança de variáveis da rede na rede. No contínuo esta condição é irrelevante, bastando que a mudança de variáveis seja não-singular. Contudo, susbiste um problema cuja origem reside no fato da transformação não ser bijetora. Para ilustrar a dificuldade, observe-se, por exemplo, que:

$$
x_{1}-x_{4}=\frac{\tau+\xi+\eta}{2} .
$$

Ainda com a presença do fator $1 / 2$ o lado direito está na rede (pois o lado esquerdo certamente está). Porém, permitindo às variáveis $\tau, \xi, \eta$ variar livremente na rede 
este já não será o caso, devendo portanto restringir-las à imagem da transformação.

Para considerar esta imagem, denotemos por $\xi_{i}, \eta_{i}, \tau_{i}$ a componente $i$-ésima de cada um dos sítios $\xi, \eta$ e $\tau$, respectivamente. Observando que se $a$ e $b$ são números inteiros quaisquer então a paridade de $a+b$ é a mesma que a de $a-b$, das definições segue que $\tau_{i}$ é par se e somente se $\xi_{i}$ e $\eta_{i}$ têm a mesma paridade e, analogamente, $\tau_{i}$ é ímpar se e somente se $\xi_{i}$ e $\eta_{i}$ têm paridades diferentes. Assim, a função característica da imagem da componente $i$-ésima da transformação é dada por:

$$
\begin{aligned}
{\left[\chi_{\text {par }}\left(\xi_{i}\right) \chi_{\text {par }}\left(\eta_{i}\right)+\chi_{\text {impar }}(\right.} & \left.\left.\xi_{i}\right) \chi_{\text {impar }}\left(\eta_{i}\right)\right] \chi_{\text {par }}\left(\tau_{i}\right) \\
& +\left[\chi_{\text {impar }}\left(\xi_{i}\right) \chi_{\text {par }}\left(\eta_{i}\right)+\chi_{\text {par }}\left(\xi_{i}\right) \chi_{\text {impar }}\left(\eta_{i}\right)\right] \chi_{\text {impar }}\left(\tau_{i}\right)
\end{aligned}
$$

onde $\chi_{\text {par }}$ e $\chi_{\text {impar }}$ denotam as funções características dos números pares e ímpares, respectivamente. Como isto é válido para cada uma das componentes, a função característica da imagem é dada por:

$$
\begin{aligned}
I(\tau, \xi, \eta):=\prod_{i=0}^{d}\left\{\left[\chi_{\text {par }}\left(\xi_{i}\right) \chi_{\text {par }}\left(\eta_{i}\right)+\chi_{\text {impar }}\left(\xi_{i}\right) \chi_{\text {impar }}\left(\eta_{i}\right)\right] \chi_{\text {par }}\left(\tau_{i}\right)\right. \\
\left.+\left[\chi_{\text {impar }}\left(\xi_{i}\right) \chi_{\text {par }}\left(\eta_{i}\right)+\chi_{\text {par }}\left(\xi_{i}\right) \chi_{\text {impar }}\left(\eta_{i}\right)\right] \chi_{\text {impar }}\left(\tau_{i}\right)\right\} .
\end{aligned}
$$

Assim, para $\tau, \xi, \eta \in \mathbb{Z}^{d+1}$ em geral devemos definir:

$$
\begin{aligned}
D(\tau, \xi, \eta) & =I(\tau, \xi, \eta) D(\tau, \xi, \eta) \\
D_{0}(\tau, \xi, \eta) & =I(\tau, \xi, \eta) D_{0}(\tau, \xi, \eta) \\
\check{K}(\tau, \xi, \eta) & =I(\tau, \xi, \eta) \check{K}(\tau, \xi, \eta) .
\end{aligned}
$$

Incidentalmente, observe-se que a função $I$ é simétrica em todas as variáveis. Ou seja, o seu valor é invariante por permutações arbitrárias das mesmas.

Para considerar a imagem da mudança de variáveis anterior à direita, observe-se que a mesma é da forma $y \longrightarrow u:=: T(y):=A y+B \operatorname{com} B=B_{1} \times B_{2}$ e $A=A_{1} \times A_{2}$, onde:

$$
A_{1}=\left(\begin{array}{cc}
-1 & -1 \\
1 & -1
\end{array}\right), \quad A_{2}=\left(\begin{array}{cc}
1 & 1 \\
1 & -1
\end{array}\right) ; \quad B_{1}=\left(x_{1}+x_{2}, 0\right), \quad B_{2}=\left(-\left(x_{3}+x_{4}\right), 0\right) .
$$

Aqui, por abuso de notação, 1 denota a matriz identidade $(d+1) \times(d+1)$. Assim, a função característica da imagem de $T$ está dada pelo produto das funções características das imagens de $T_{1}$ e $T_{2}$ :

$$
\chi_{T}\left(u_{1}, u_{2}, u_{3}, u_{4}\right)=\chi_{T_{1}}\left(u_{1}, u_{2}\right) \chi_{T_{2}}\left(u_{3}, u_{4}\right),
$$


onde denotamos $T_{i}(y):=A_{i} y+B_{i}, i=1,2$. Analogamente ao caso anterior, ou apenas observando que os conjuntos de variáves $\left(u_{1}, \xi, u_{2}\right)$ e $\left(u_{3}, u_{4}, \eta\right)$ são formalmente análogos a $(\tau, \xi, \eta)$, é simples verificar que:

$$
\begin{aligned}
& \chi_{T_{1}}\left(u_{1}, u_{2}\right)=I\left(u_{1}, \xi, u_{2}\right) \\
& \chi_{T_{2}}\left(u_{3}, u_{4}\right)=I\left(u_{3}, u_{4}, \eta\right) .
\end{aligned}
$$

Em termos das novas variáveis, (3.2.1) resulta em:

$$
\begin{aligned}
& D(\tau, \xi, \eta) \\
& =D_{0}(\tau, \xi, \eta)- \\
& \sum_{u_{1}, u_{2}, u_{3}, u_{4} \in \mathbb{Z}^{d+1}} \chi_{T}\left(u_{1}, u_{2}, u_{3}, u_{4}\right) D_{0}\left(u_{1}, \xi, u_{2}\right) \check{K}\left(\tau-u_{1}-u_{3}, u_{2}, u_{4}\right) D\left(u_{3}, u_{4}, \eta\right) .
\end{aligned}
$$

Agora, se denotamos $R:=\widehat{D}$ e $R_{0}:=\widehat{D}_{0}$, tomando a transformada de Fourier da identidade acima, temos, após da mudança de variáveis $\tau^{\prime}:=\tau-u_{1}-u_{3}$ e um reagrupamento das somatórias, que:

$$
\begin{aligned}
& R(k, p, q) \\
& =R_{0}(k, p, q)-(2 \pi)^{-\frac{3(d+1)}{2}} \sum_{\tau^{\prime}, u_{2}, u_{4} \in \mathbb{Z}^{d+1}} \mathrm{e}^{-i k \cdot \tau^{\prime} \check{K}\left(\tau^{\prime}, u_{2}, u_{4}\right)} \\
& \quad \times\left(\sum_{\xi, u_{1} \in \mathbb{Z}^{d+1}} \mathrm{e}^{-i k \cdot u_{1}} \mathrm{e}^{-i p \cdot \xi} \chi_{T_{1}}\left(u_{1}, u_{2}, \xi\right) D_{0}\left(u_{1}, \xi, u_{2}\right)\right) \\
& \quad \times\left(\sum_{\eta, u_{3} \in \mathbb{Z}^{d+1}} \mathrm{e}^{-i k \cdot u_{3}} \mathrm{e}^{-i q \cdot \eta} \chi_{T_{2}}\left(u_{3}, u_{4}, \eta\right) D\left(u_{3}, u_{4}, \eta\right)\right) .
\end{aligned}
$$

Se escrevemos $D_{0}$ como sendo a antitransformada de Fourier de $R_{0}$, temos que, após trocar a ordem da somatória com as integrais, o primeiro fator entre parênteses dentro da somatória acima resulta em:

$$
\begin{aligned}
(2 \pi)^{-\frac{3(d+1)}{2}} \int_{T^{d+1}}\left(\sum_{\xi, u_{1} \in \mathbb{Z}^{d+1}} \mathrm{e}^{-i(p-\beta) \cdot \xi} \mathrm{e}^{-i(k-\alpha) \cdot u_{1}} I\left(u_{1}, \xi, u_{2}\right)\right) & \\
& \times \mathrm{e}^{i u_{2} \cdot \gamma} R_{0}(\alpha, \beta, \gamma) d \alpha d \beta d \gamma
\end{aligned}
$$


Se denotamos:

$$
\begin{aligned}
\lambda_{i}:=\mathrm{e}^{-i\left(p_{i}-\beta_{i}\right) \xi_{i}} \mathrm{e}^{-i\left(k_{i}-\alpha_{i}\right) u_{1}^{i}} & \\
& \times\left\{\left[\chi_{\text {par }}\left(\xi_{i}\right) \chi_{\text {par }}\left(u_{2}^{i}\right)+\chi_{\text {impar }}\left(\xi_{i}\right) \chi_{\text {impar }}\left(u_{2}^{i}\right)\right] \chi_{\text {par }}\left(u_{1}^{i}\right)\right. \\
& \left.\quad+\left[\chi_{\text {impar }}\left(\xi_{i}\right) \chi_{\text {par }}\left(u_{2}^{i}\right)+\chi_{\text {par }}\left(\xi_{i}\right) \chi_{\text {impar }}\left(u_{2}^{i}\right)\right] \chi_{\text {impar }}\left(u_{1}^{i}\right)\right\},
\end{aligned}
$$

onde $u_{1}^{i}$ e $u_{2}^{i}$ denotam a componente $i$-ésima de $u_{1}$ e $u_{2}$, respectivamente, o factor entre parênteses no integrando em (3.2.3) é dado por:

$$
\sum_{\xi_{0} \in \mathbb{Z}} \cdots \sum_{\xi_{d} \in \mathbb{Z}} \sum_{u_{1}^{0} \in \mathbb{Z}} \cdots \sum_{u_{1}^{d} \in \mathbb{Z}} \prod_{i=0}^{d} \lambda_{i}=\prod_{i=0}^{d}\left(\sum_{\xi_{i} \in \mathbb{Z}} \sum_{u_{1}^{i} \in \mathbb{Z}} \lambda_{i}\right)
$$

pois cada $\lambda_{i}$ depende apenas de $\xi_{i}$ e $u_{1}^{i}$, e assim (3.2.3) fica:

$$
\begin{aligned}
(2 \pi)^{-\frac{3(d+1)}{2}} \int_{T^{d+1}} d & \gamma \mathrm{e}^{i u_{2} \cdot \gamma} \int_{-\pi}^{\pi} d \alpha_{0} \cdots \int_{-\pi}^{\pi} d \alpha_{d} \\
& \times \int_{-\pi}^{\pi} d \beta_{0} \cdots \int_{-\pi}^{\pi} d \beta_{d} \prod_{i=0}^{d}\left(\sum_{\xi_{i} \in \mathbb{Z}} \sum_{u_{1}^{i} \in \mathbb{Z}} \lambda_{i}\right) R_{0}(\alpha, \beta, \gamma) .
\end{aligned}
$$


Agora,

$$
\begin{aligned}
& \sum_{\xi_{i} \in \mathbb{Z}} \sum_{u_{1}^{i} \in \mathbb{Z}} \lambda_{i} \\
& =\sum_{\substack{u_{1}^{i} \in \mathbb{Z} \\
u_{1}^{i} \text { par }}} \mathrm{e}^{-i\left(k_{i}-\alpha_{i}\right) u_{1}^{i}} \sum_{\xi_{i} \in \mathbb{Z}} \mathrm{e}^{-i\left(p_{i}-\beta_{i}\right) \xi_{i}}\left[\chi_{\text {par }}\left(\xi_{i}\right) \chi_{\text {par }}\left(u_{2}^{i}\right)+\chi_{\text {impar }}\left(\xi_{i}\right) \chi_{\text {impar }}\left(u_{2}^{i}\right)\right] \\
& +\sum_{u_{1}^{i} \in \mathbb{Z}} \mathrm{e}^{-i\left(k_{i}-\alpha_{i}\right) u_{1}^{i}} \sum_{\xi_{i} \in \mathbb{Z}} \mathrm{e}^{-i\left(p_{i}-\beta_{i}\right) \xi_{i}}\left[\chi_{\text {impar }}\left(\xi_{i}\right) \chi_{\text {par }}\left(u_{2}^{i}\right)+\chi_{\text {par }}\left(\xi_{i}\right) \chi_{\text {impar }}\left(u_{2}^{i}\right)\right] \\
& u_{1}^{i} \text { impar } \\
& =\sum_{\substack{u_{1}^{i} \in \mathbb{Z} \\
u_{1}^{i} \text { par }}} \mathrm{e}^{-i\left(k_{i}-\alpha_{i}\right) u_{1}^{i}}\left[\chi_{\text {par }}\left(u_{2}^{i}\right) \sum_{n \in \mathbb{Z}} \mathrm{e}^{-i\left(p_{i}-\beta_{i}\right) 2 n}\right. \\
& \left.+\chi_{\text {impar }}\left(u_{2}^{i}\right) \sum_{n \in \mathbb{Z}} \mathrm{e}^{-i\left(p_{i}-\beta_{i}\right)} \mathrm{e}^{-i\left(p_{i}-\beta_{i}\right) 2 n}\right] \\
& +\sum_{\substack{u_{1}^{i} \in \mathbb{Z} \\
u_{1}^{i} \text { impar }}} \mathrm{e}^{-i\left(k_{i}-\alpha_{i}\right) u_{1}^{i}}\left[\chi_{\text {par }}\left(u_{2}^{i}\right) \sum_{n \in \mathbb{Z}} \mathrm{e}^{-i\left(p_{i}-\beta_{i}\right)} \mathrm{e}^{-i\left(p_{i}-\beta_{i}\right) 2 n}\right. \\
& \left.+\chi_{\text {impar }}\left(u_{2}^{i}\right) \sum_{n \in \mathbb{Z}} \mathrm{e}^{-i\left(p_{i}-\beta_{i}\right) 2 n}\right] \\
& =\sum_{\substack{u_{1}^{i} \in \mathbb{Z} \\
u_{1}^{i} \text { par }}} \mathrm{e}^{-i\left(k_{i}-\alpha_{i}\right) u_{1}^{i}}\left[\chi_{\text {par }}\left(u_{2}^{i}\right)+\chi_{\text {impar }}\left(u_{2}^{i}\right) \mathrm{e}^{-i\left(p_{i}-\beta_{i}\right)}\right] \sum_{n \in \mathbb{Z}} \mathrm{e}^{-i\left(p_{i}-\beta_{i}\right) 2 n} \\
& +\sum_{\substack{u_{1}^{i} \in \mathbb{Z} \\
u_{1}^{i} \text { impar }}} \mathrm{e}^{-i\left(k_{i}-\alpha_{i}\right) u_{1}^{i}}\left[\chi_{\text {par }}\left(u_{2}^{i}\right) \mathrm{e}^{-i\left(p_{i}-\beta_{i}\right)}+\chi_{\text {impar }}\left(u_{2}^{i}\right)\right] \sum_{n \in \mathbb{Z}} \mathrm{e}^{-i\left(p_{i}-\beta_{i}\right) 2 n} \\
& =\sum_{\substack{u_{1}^{i} \in \mathbb{Z} \\
u_{1}^{i} \text { par }}} \mathrm{e}^{-i\left(k_{i}-\alpha_{i}\right) u_{1}^{i}}\left[\chi_{\text {par }}\left(u_{2}^{i}\right)+\chi_{\text {impar }}\left(u_{2}^{i}\right) \mathrm{e}^{-i\left(p_{i}-\beta_{i}\right)}\right] \pi \delta\left(p_{i}-\beta_{i}\right) \\
& +\sum_{\substack{u_{1}^{i} \in \mathbb{Z} \\
u_{1}^{i} \text { impar }}} \mathrm{e}^{-i\left(k_{i}-\alpha_{i}\right) u_{1}^{i}}\left[\chi_{\text {par }}\left(u_{2}^{i}\right) \mathrm{e}^{-i\left(p_{i}-\beta_{i}\right)}+\chi_{\text {impar }}\left(u_{2}^{i}\right)\right] \pi \delta\left(p_{i}-\beta_{i}\right) .
\end{aligned}
$$

Se substituimos o fator entre parênteses em (3.2.4) com esta última expressão, após 
integrar em $d \beta$ obtemos:

$$
\begin{gathered}
(2 \pi)^{-\frac{3(d+1)}{2}} \pi^{d+1} \int_{T^{d+1}} d \gamma \mathrm{e}^{i u_{2} \cdot \gamma} \int_{-\pi}^{\pi} d \alpha_{0} \ldots \\
\cdots \int_{-\pi}^{\pi} d \alpha_{d} \prod_{i=0}^{d}\left(\sum_{u_{1}^{i} \in \mathbb{Z}} \mathrm{e}^{-i\left(k_{i}-\alpha_{i}\right) u_{1}^{i}}\right) R_{0}(\alpha, p, \gamma) \\
=(2 \pi)^{-\frac{3(d+1)}{2}} \pi^{d+1} \int_{T^{d+1}} d \gamma \mathrm{e}^{i u_{2} \cdot \gamma} \int_{-\pi}^{\pi} d \alpha_{0} \cdots \int_{-\pi}^{\pi} d \alpha_{d} \prod_{i=0}^{d} 2 \pi \delta\left(k_{i}-\alpha_{i}\right) R_{0}(\alpha, p, \gamma) \\
=(2 \pi)^{-\frac{3(d+1)}{2}} \pi^{d+1}(2 \pi)^{d+1} \int_{T^{d+1}} d \gamma \mathrm{e}^{i u_{2} \cdot \gamma} R_{0}(k, p, \gamma) \\
=\left(\frac{\pi}{2}\right)^{\frac{d+1}{2}} \int_{T^{d+1}} \mathrm{e}^{i u_{2} \cdot \gamma} R_{0}(k, p, \gamma) d \gamma .
\end{gathered}
$$

Esta expressão, lembre-se, corresponde a (3.2.3), que pela sua vez corresponde ao primeiro fator entre parênteses em (3.2.2). Analogamente, o segundo fator entre parênteses em (3.2.2) é dado por:

$$
\left(\frac{\pi}{2}\right)^{\frac{d+1}{2}} \int_{T^{d+1}} \mathrm{e}^{i u_{4} \cdot \beta} R(k, \beta, q) d \beta
$$

Introduzindo (3.2.5) e (3.2.6) em (3.2.2) e trocando a ordem entre somatória e integrais temos:

$$
R(k, p, q)=R_{0}(k, p, q)-\int_{T^{d+1}} \int_{T^{d+1}} R_{0}(k, p, \alpha) K(k, \alpha, \beta) R(k, \beta, q) d \alpha d \beta
$$

Aqui, por abuso de notação e linguagem, adotamos:

$$
K(k, \alpha, \beta):=4^{-(d+1)}(2 \pi)^{-\frac{d+1}{2}} K(k,-\alpha,-\beta) .
$$

Observe que as propriedades de analiticidade das funções a esquerda e a direita acima serão basicamente as mesmas. Se em $L^{2}\left(T^{d+1}, d x\right)$ definimos os operadores integrais $A(k)$ por:

$$
(A(k)(f))(p):=\int_{T^{d+1}} A(k, p, q) f(q) d q
$$

onde $A=R, R_{0}, K$, podemos reescrever (3.2.7) como:

$$
R(k)=R_{0}(k)-R_{0}(k) K(k) R(k) .
$$


A identidade (3.2.7) e o análogo na rede da expressão (2.6) no trabalho de Spencer e Zirilli, [101] p. 4. Observemos também que:

$$
\begin{aligned}
& R_{0}(k, p, q) \\
& =(2 \pi)^{-\frac{3(d+1)}{2}} \sum_{\tau, \xi, \eta \in \mathbb{Z}^{d+1}} \mathrm{e}^{-i(k \cdot \tau+p \cdot \xi+q . \eta)} I(\tau, \xi, \eta) S_{2}\left(\frac{\tau+(\xi-\eta)}{2}\right) \\
& +(2 \pi)^{-\frac{3(d+1)}{2}} \sum_{\tau, \xi, \eta \in \mathbb{Z}^{d+1}} \mathrm{e}^{-i(k \cdot \tau+p \cdot \xi+q . \eta)} I(\tau, \xi, \eta) S_{2}\left(\frac{\tau-(\xi-\eta)}{2}\right) \\
& 2
\end{aligned}
$$

Agora, no primeiro somando efetuamos a mudança de variáveis dada por:

$$
\begin{aligned}
\tau & =u+v \\
\xi & =u-v+w \\
\eta & =w .
\end{aligned}
$$

Em termos destas novas variáveis tem-se:

$$
\begin{aligned}
& I(\tau, \xi, \eta)=\prod_{i=0}^{d}\left\{\left[\chi_{\text {par }}\left(u_{i}-v_{i}+w_{i}\right) \chi_{\text {par }}\left(w_{i}\right)\right.\right. \\
& \left.+\chi_{\text {impar }}\left(u_{i}-v_{i}+w_{i}\right) \chi_{\text {impar }}\left(w_{i}\right)\right] \chi_{p a r}\left(u_{i}+v_{i}\right) \\
& \left.\left.+\left[\chi_{\text {impar }}\left(u_{i}-v_{i}+w_{i}\right) \chi_{\text {par }}\left(w_{i}\right)+w_{i}\right) \chi_{\text {impar }}\left(w_{i}\right)\right] \chi_{\text {impar }}\left(u_{i}+v_{i}\right)\right\}=: J(u, v, w) . \\
& +\chi_{\text {par }}\left(u_{i}-v_{i}+w\right)
\end{aligned}
$$

Agora, observe que $u_{i}+v_{i}$ é par se e somente se $u_{i}$ e $v_{i}$ têm a mesma paridade, e neste caso $u_{i}-v_{i}+w_{i}$ e $w_{i}$ terão a mesma paridade (que será paridade de $w_{i}$ ). Analogamente, $u_{i}+v_{i}$ é ímpar se e somente se $u_{i}$ e $v_{i}$ têm paridade diferente, em cujo caso $u_{i}-v_{i}+w_{i}$ e $w_{i}$ terão paridade diferente, independentemente da paridade de $w_{i}$. Portanto, a função $J$ acima definida é identicamente igual a 1 :

$$
J(u, v, w)=1 .
$$

(Observe-se que $I(\tau, \xi, \eta)$ não é identicamente igual a 1, pois as três variáveis são independentes. No caso de $J(u, v, w)$, embora ela também seja função de três variáves independentes, dois de elas não aparecem senão na combinação $u+v$ ou $u-v$, que 
têm a mesma paridade. Assim, aos fines da paridade ficam somente duas variáveis independentes. É por isso que os valores de $J(u, v, w)$ são restritos, ao ponto de ser identicamente igual a 1). Com estas observações, o primeiro somando anterior resulta em:

$$
\begin{aligned}
(2 \pi)^{-\frac{3(d+1)}{2}} \sum_{u, v, w \in \mathbb{Z}^{d+1}} \mathrm{e}^{-i(k+p) \cdot u} \mathrm{e}^{-i(k-p) \cdot v} \mathrm{e}^{-i(p+q) \cdot w} S_{2}(u) S_{2}(v) & \\
& =(2 \pi)^{\frac{d+1}{2}} \delta(p+q) \widehat{S}_{2}(k+p) \widehat{S}_{2}(k-p) .
\end{aligned}
$$

O segundo somando pode ser tratado analogamente, com a mudança de variáveis dada por:

$$
\begin{aligned}
\tau & =u+v \\
\xi & =u-v-w \\
\eta & =w,
\end{aligned}
$$

obtendo-se neste caso:

$$
(2 \pi)^{\frac{d+1}{2}} \delta(p-q) \widehat{S}_{2}(k+p) \widehat{S}_{2}(k-p) .
$$

Assim:

$$
R_{0}(k, p, q)=(2 \pi)^{\frac{d+1}{2}} \widehat{S}_{2}(k+p) \widehat{S}_{2}(k-p)[\delta(p+q)+\delta(p-q)] .
$$

Em particular, agindo sobre funções simétricas, i.e., com $f(p)=f(-p)$, o operador integral $R_{0}(k)$ está dado pelo núcleo:

$$
R_{0}(k, p, q)=R_{0}(k, p) \delta(p+q),
$$

onde:

$$
R_{0}(k, p):=2(2 \pi)^{\frac{d+1}{2}} \widehat{S}_{2}(k+p) \widehat{S}_{2}(k-p) .
$$

Esta identidade exibe total analogia com a expressão (2.5) de Spencer e Zirilli, [101] p. 4.

\subsection{Relação entre Resolvente e Espectro}

Se $R(\lambda):=(A-\lambda I)^{-1}$ denota o resolvente de um operador autoadjunto $A$ e $E_{t}$ a sua família espectral, basicamente do teorema espectral temos: ${ }^{17}$

$$
\langle f, R(\lambda) g\rangle=\int_{-\infty}^{+\infty} \frac{1}{t-\lambda} d\left\langle f, E_{t} g\right\rangle \forall f, g .
$$

\footnotetext{
${ }^{17}[79]$ p. 173.
} 
Esta fórmula estabelece um nexo entre a medida espectral do operador e o seu resolvente. Estabeleceremos a seguir uma identidade que permite reconhecer o operador $R(k)$ da seção anterior (ou seja, a tranformada de Fourier de $D$ ) como uma espécie de resolvente para o operador de energia-momento. Esta analogia é no sentido da identidade acima, mas de uma maneira parcial, já que no presente caso não resultará válida para todos os vetores, mas apenas para os "estados de duas partículas".

Das definições temos que:

$$
\begin{array}{r}
D(\tau, \xi, \eta)=S_{4}\left(\frac{\tau+\xi+\eta}{2}, \frac{\tau-\xi+\eta}{2}, \eta\right)-S_{2}(\xi) S_{2}(\eta) \\
=\mu\left(p_{\frac{\tau+\xi+\eta}{2}} p_{\frac{\tau-\xi+\eta}{2}} p_{\eta} p_{0}\right)-\mu\left(p_{\xi} p_{0}\right) \mu\left(p_{\eta} p_{0}\right) \\
=\mu\left(\left(p_{\xi} p_{0}-\mu\left(p_{\xi} p_{0}\right) \mathbb{I}\right) \cdot(\mathbb{I}) \cdot \tau_{-\frac{\tau-\xi+\eta}{2}}\left(p_{\eta} p_{0}-\mu\left(p_{\eta} p_{0}\right) \mathbb{I}\right) \cdot(\mathbb{I})\right) \\
\quad=\left\langle\left(P_{\xi} P_{0}-\mu\left(p_{\xi} p_{0}\right) I_{\mathcal{H}}\right) \Omega, T_{-\frac{\tau-\xi+\eta}{2}}\left(P_{\eta} P_{0}-\mu\left(p_{\eta} p_{0}\right) I_{\mathcal{H}}\right) \Omega\right\rangle_{\mathcal{H}}
\end{array}
$$

onde para a última igualdade temos suposto que $\xi_{0}=0$. Supondo agora que também $\eta_{0}=0$, isto pode escrever-se como:

$$
D(\tau, \xi, \eta)=\left\langle\Theta(\xi), \mathrm{e}^{-\frac{1}{2}\left|\tau_{0}\right| H} \mathrm{e}^{-\frac{1}{2} i \tau . \mathrm{P}} \Theta(\eta)\right\rangle_{\mathcal{H}},
$$

onde:

$$
\Theta(\alpha):=\mathrm{e}^{-\frac{1}{2} i \alpha \cdot \mathrm{P}}\left(P_{\alpha} P_{0}-\mu\left(p_{\alpha} p_{0}\right) I_{\mathcal{H}}\right) \Omega .
$$

Sejam agora $f$ e $g$ duas funções de quadrado integrável, com dependência apenas espacial, i.e., $f\left(p_{0}, \mathrm{p}\right)=f(\mathrm{p}) \in L^{2}\left(T^{d+1}, d x\right)$ e analogamente para $g$, e simétricas. Temos:

$$
\begin{aligned}
& \langle f, R(k) g\rangle_{L^{2}\left(T^{d+1}\right)}=\int_{T^{d+1}} \int_{T^{d+1}} \widehat{D}(k, p, q) \overline{f(p)} g(q) d q d p \\
& =(2 \pi)^{\frac{1-d}{2}} \sum_{\tau,(0, \xi),(0, \eta)} \mathrm{e}^{-i k \cdot \tau} D(\tau,(0, \xi),(0, \eta)) \check{\bar{f}}(-\xi) \check{g}(-\eta) \\
& =(2 \pi)^{\frac{1-d}{2}} \sum_{\tau,(0, \xi),(0, \eta)} \mathrm{e}^{-i k \cdot \tau}\left\langle\Theta(\xi), \mathrm{e}^{-\frac{1}{2}\left|\tau_{0}\right| H} \mathrm{e}^{-\frac{1}{2} i \tau \cdot \mathrm{P}} \Theta(\eta)\right\rangle_{\mathcal{H}} \check{\bar{f}}(-\xi) \check{g}(-\boldsymbol{\eta}) \\
& =(2 \pi)^{\frac{1-d}{2}} \sum_{\tau} \mathrm{e}^{-i k \cdot \tau}\left\langle\Theta(\check{f}), \mathrm{e}^{-\frac{1}{2}\left|\tau_{0}\right| H} \mathrm{e}^{-\frac{1}{2} i \tau \cdot \mathrm{P}} \Theta(\check{g})\right\rangle_{\mathcal{H}} \quad=:(*),
\end{aligned}
$$

onde denotamos:

$$
\Theta(h):=\sum_{x} \Theta(x) h(-x) .
$$


Continuando, usando agora a medida espectral de $(H, \mathbf{P})$, temos:

$$
\begin{aligned}
(*)=(2 \pi)^{\frac{1-d}{2}} \int_{0}^{\infty} \int_{T^{d}}\left(\sum_{\tau_{0} \in \mathbb{Z}} \mathrm{e}^{-i k_{0} \tau_{0}} \mathrm{e}^{-\frac{1}{2}\left|\tau_{0}\right| \lambda_{0}}\right) \\
\times\left(\sum_{\tau \in \mathbb{Z}^{d}} \mathrm{e}^{-i \mathrm{k} \cdot \boldsymbol{\tau}} \mathrm{e}^{-\frac{1}{2} i \tau . \lambda}\right) d\left\langle\Theta(\check{f}), E_{\lambda} \Theta(\check{g})\right\rangle_{\mathcal{H}} \\
=(2 \pi)^{\frac{d+1}{2}} \int_{0}^{\infty} \int_{T^{d}} \operatorname{Re}\left(\frac{1+\mathrm{e}^{-\left(\frac{\lambda_{0}}{2}+i k_{0}\right)}}{\left.1-\mathrm{e}^{-\left(\frac{\lambda_{0}}{2}+i k_{0}\right)}\right) \delta\left(\frac{\lambda}{2}+\mathrm{k}\right) d\left\langle\Theta(\check{f}), E_{\lambda} \Theta(\check{g})\right\rangle_{\mathcal{H}} .}\right.
\end{aligned}
$$

Ou seja:

$$
\begin{aligned}
& \langle f, R(k) g\rangle_{L^{2}\left(T^{d+1}\right)} \\
& \quad=(2 \pi)^{\frac{d+1}{2}} \int_{0}^{\infty} \int_{T^{d}} \operatorname{Re}\left(\frac{1+\mathrm{e}^{-\left(\frac{\lambda_{0}}{2}+i k_{0}\right)}}{1-\mathrm{e}^{-\left(\frac{\lambda_{0}}{2}+i k_{0}\right)}}\right) \delta\left(\frac{\lambda}{2}+\mathrm{k}\right) d\left\langle\Theta(\check{f}), E_{\lambda} \Theta(\check{g})\right\rangle_{\mathcal{H}} .
\end{aligned}
$$

Esta identidade permite transcrever propriedades de $R(k)$ em propriedades do suporte da medida espectral (e portanto, do espectro) do operador de energia-momento, constituindo o análogo na rede da expressão (5.1) no trabalho de Spencer e Zirilli, [101] p. $13 .{ }^{18}$ Devido à presença da $\delta$ no integrando, este método não permite estudar o espectro conjunto do operador de energia-momento, senão o espectro de energia com momento (arbitrário, em principio, porém) fixo. Observe-se também que esta identidade é válida apenas para os vetores da forma $\Theta(\check{f}$ ) (basicamente, "estados de duas partículas"), permitindo portando obter informação apenas na parte de baixas energias do espectro. Resulta digno de destacar que a identidade acima não depende do modelo nem de nenhuma hipótese especial sobre as funções de $n$ pontos. A fórmula de Gellmann-Low e a última identidade da seção 2.1, p. 22 , conduzem a fórmulas análogas, relacionando a função de 2 pontos e os estados de uma partícula. Certamente, o estudo do espectro para energias maiores requer o uso de núcleos de ordem superior. ${ }^{19}$

\footnotetext{
${ }^{18}$ Cf. [101] p. 4.

${ }^{19}[21]$, [68]. Núcleos de ordem superior são considerados detalhadamente em [20]; Cf. [32].
} 
Denotando, analogamente a [84] p. 220, $a:=\cosh \left(\lambda_{0} / 2\right)-1$ temos:

$$
\begin{gathered}
\operatorname{Re}\left(\frac{1+\mathrm{e}^{-\frac{\lambda_{0}}{2}} \mathrm{e}^{-i k_{0}}}{1-\mathrm{e}^{-\frac{\lambda_{0}}{2}} \mathrm{e}^{-i k_{0}}}\right)=\frac{1-\mathrm{e}^{-\lambda_{0}}}{1-2 \mathrm{e}^{-\frac{\lambda_{0}}{2}} \cos k_{0}+\mathrm{e}^{-\lambda_{0}}} \\
=\frac{\frac{\mathrm{e}^{\frac{\lambda_{0}}{2}}-\mathrm{e}^{-\frac{\lambda_{0}}{2}}}{2}}{\frac{\mathrm{e}^{\frac{\lambda_{0}}{2}}+\mathrm{e}^{-\frac{\lambda_{0}}{2}}}{2}-\cos k_{0}}=\frac{\operatorname{senh} \lambda_{0} / 2}{\cosh \lambda_{0} / 2-\cos k_{0}}=\frac{\sqrt{(a+1)^{2}-1}}{a-\left(\cos k_{0}-1\right)} .
\end{gathered}
$$

Seja $h \in \mathcal{D}(0,+\infty)$. Introduzindo (3.3.2) em (3.3.1) e denotando agora $\cos k_{0}-$ $1=: x+i y$, tem-se:

$$
\begin{aligned}
& \lim _{y \rightarrow 0^{+}} \int_{0}^{\infty} \operatorname{Im}\langle f,\left.R\left(\left(k_{0}, \mathrm{k}\right)\right) g\right\rangle h(x) d x \\
&=(2 \pi)^{\frac{d+1}{2}} \int_{0}^{\infty} \lim _{y \rightarrow 0^{+}} \int_{0}^{\infty} \frac{y}{(a-x)^{2}+y^{2}} h(x) d x d \nu(a) \\
&=(2 \pi)^{\frac{d+1}{2}} \pi \int_{0}^{\infty} h(a) d \nu(a),
\end{aligned}
$$

onde denotamos:

$$
d \nu(a):=\sqrt{(a+1)^{2}-1} d\left\langle\Theta(\check{f}), E_{(2 \operatorname{arcosh}(a+1),-2 \mathbf{k})} \Theta(\check{g})\right\rangle_{\mathcal{H}}
$$

A identidade (3.3.3) basicamente prova o seguinte

3.3.1 Lema: Seja k arbitrário, mas fixo. Se a distribuição definida em $\mathcal{D}(0,+\infty)$ por:

$$
h \longmapsto \lim _{y \rightarrow 0^{+}} \int_{0}^{\infty} \operatorname{Im}\left\langle f, R\left(\left(k_{0}, \mathbf{k}\right)\right) g\right\rangle h(x) d x
$$

anula-se no aberto $(\alpha, \beta)$, então a medida $d\left\langle\Theta(\check{f}), E_{\left(\lambda_{0},-2 \mathbf{k}\right)} \Theta(\check{g})\right\rangle_{\mathcal{H}}$ anula-se no aberto $(2 \operatorname{arcosh}(\alpha+1), 2 \operatorname{arcosh}(\beta+1))$.

Demonstração: Seja $f \in \mathcal{D}(0,+\infty)$ com supp $f \subset(2 \operatorname{arcosh}(\alpha+1), 2 \operatorname{arcosh}(\beta+1))$. Definindo:

$$
h(a):= \begin{cases}\frac{f(2 \operatorname{arcosh}(a+1))}{\sqrt{(a+1)^{2}-1}} & \text { se } a \in(\alpha, \beta) \\ 0 & \text { se } a \in(0,+\infty) \backslash(\alpha, \beta)\end{cases}
$$


e usando (3.3.3) tem-se:

$$
\begin{aligned}
& \int_{0}^{\infty} f\left(\lambda_{0}\right) d\left\langle\Theta(\check{f}), E_{\left(\lambda_{0},-2 \mathbf{k}\right)} \Theta(\check{g})\right\rangle_{\mathcal{H}}=\int_{0}^{\infty} h(a) d \nu(a) \\
&=\mathrm{const} \lim _{y \rightarrow 0^{+}} \int_{0}^{\infty} \operatorname{Im}\left\langle f, R\left(\left(k_{0}, \mathbf{k}\right)\right) g\right\rangle h(x) d x \\
&=\text { const } \lim _{y \rightarrow 0^{+}} \int_{\alpha}^{\beta} \operatorname{Im}\left\langle f, R\left(\left(k_{0}, \mathbf{k}\right)\right) g\right\rangle h(x) d x,
\end{aligned}
$$

onde a última integral é nula por hipótese.

A identidade (3.3.3) e o lema 3.3.1 constituem os análogos na rede da expressão (5.2) e o lema 5.1, respectivamente, no trabalho de Spencer e Zirilli, [101] p. 13.

\subsection{Propriedades de Regularidade dos Operado- res $R_{0}(k)$ e $K(k)$}

Introduzimos as seguintes hipóteses:

H1

$$
\widehat{S}_{2}\left(p_{0}, \mathbf{p}\right)=Z(\mathbf{p}) \operatorname{Re}\left(\frac{1+\mathrm{e}^{-\left(\omega(\mathbf{p})+i p_{0}\right)}}{1-\mathrm{e}^{-\left(\omega(\mathbf{p})+i p_{0}\right)}}\right)+\int_{m+2 \delta_{0}}^{\infty} \operatorname{Re}\left(\frac{1+\mathrm{e}^{-\left(\lambda_{0}+i p_{0}\right)}}{1-\mathrm{e}^{-\left(\lambda_{0}+i p_{0}\right)}}\right) d \rho\left(\lambda_{0}, \mathbf{p}\right)
$$

onde $Z(\mathrm{p})$ é uma função $C^{\infty}$ positiva, $\omega(\mathrm{p})$ é uma função real analítica com $m \leqslant \omega(\mathrm{p})<m+2 \delta_{0}$ e $\delta_{0}>0$.

H2 $K(k, p, q)$ é analítico na região:

$$
\begin{aligned}
\left|\operatorname{Im} p_{i}\right| & <\delta_{i}(i=0,1, \ldots, d) \\
\left|\operatorname{Im} q_{i}\right| & <\delta_{i}(i=0,1, \ldots, d) \\
\left|\operatorname{Im} k_{0}\right| & <m+\delta_{0},
\end{aligned}
$$

para certos $\delta_{i}>0(i=1, \ldots, d)$.

A hipótese $\mathrm{H} 1$ é consequência do gap (ou lacuna) de massa inferior e superior no espectro do operador de enegia-momento ${ }^{20}$ e foi verificada para diversos modelos na ${ }^{20}$ Cf. AR4 na p. 26. 
rede: o caso do modelo de Ising na região de baixas temperaturas foi considerado em [84] (Cf. [70], [85]) e basicamente a mesma prova aplica-se ao caso de altas temperaturas, [69], porém as constantes $\delta_{0}$ diferem. Este último caso será considerado com detalhe na segunda parte do presente trabalho (veja-se 5.2.5(c)), onde verificaremos que a hipótese $\mathrm{H} 2$ também é satisfeita neste modelo (veja-se 6.4.4), com $\delta_{i}=\delta_{0}=m$, como conseqüência do decaimento exponencial do núcleo de Bethe-Salpeter. ${ }^{21}$

Para facilitar a prova do principal resultado desta seção, a saber, o lema 3.4.2, começamos introduzindo definições e resultados prévios. Seja:

$$
\begin{aligned}
& R_{00}(k, p) \\
& :=Z(\mathrm{p}+\mathrm{k}) \operatorname{Re}\left(\frac{1+\mathrm{e}^{-\left(\omega(\mathrm{p}+\mathrm{k})+i\left(p_{0}+k_{0}\right)\right)}}{1-\mathrm{e}^{-\left(\omega(\mathrm{p}+\mathrm{k})+i\left(p_{0}+k_{0}\right)\right)}}\right) Z(\mathrm{p}-\mathrm{k}) \operatorname{Re}\left(\frac{1+\mathrm{e}^{-\left(\omega(\mathrm{p}-\mathrm{k})+i\left(p_{0}-k_{0}\right)\right)}}{1-\mathrm{e}^{-\left(\omega(\mathrm{p}-\mathrm{k})+i\left(p_{0}-k_{0}\right)\right)}}\right) .
\end{aligned}
$$

Observe que isto pode escrever-se como:

$$
R_{00}(k, p)=\frac{Z_{1}(\mathrm{p}+\mathrm{k})}{\cosh \omega(\mathrm{p}+\mathrm{k})-\cos \left(p_{0}+k_{0}\right)} \frac{Z_{1}(\mathrm{p}-\mathrm{k})}{\cosh \omega(\mathrm{p}-\mathrm{k})-\cos \left(p_{0}-k_{0}\right)},
$$

onde $Z_{1}(p):=Z(\mathrm{p}) \operatorname{senh} \omega(\mathrm{p})$, ou, alternativamente, como:

$$
\begin{aligned}
& R_{00}(k, p)=\frac{Z_{1}(\mathrm{p}+\mathrm{k}) Z_{1}(\mathrm{p}-\mathrm{k})}{[\cosh \omega(\mathrm{p}-\mathrm{k})-\cosh \omega(\mathrm{p}+\mathrm{k})]-2 \operatorname{sen} k_{0} \operatorname{sen} p_{0}} \\
& \quad \times\left(\frac{1}{\cosh \omega(\mathrm{p}+\mathrm{k})-\cos \left(p_{0}+k_{0}\right)}-\frac{1}{\cosh \omega(\mathrm{p}-\mathrm{k})-\cos \left(p_{0}-k_{0}\right)}\right) .
\end{aligned}
$$

\subsubsection{Lema:}

(a) Se $\left|\operatorname{Im} k_{0}\right|<m$, então:

$$
\begin{aligned}
\int_{-\pi}^{\pi} & \frac{1}{\cosh \omega(\mathrm{p}+\mathrm{k})-\cos \left(p_{0}+k_{0}\right)} \frac{1}{\cosh \omega(\mathrm{p}-\mathrm{k})-\cos \left(p_{0}-k_{0}\right)} d p_{0} \\
& =\frac{2 \pi \operatorname{senh}[\omega(\mathrm{p}+\mathrm{k})+\omega(\mathrm{p}-\mathrm{k})]}{\operatorname{senh} \omega(\mathrm{p}+\mathrm{k}) \operatorname{senh} \omega(\mathrm{p}-\mathrm{k})\left(\cosh [\omega(\mathrm{p}+\mathrm{k})+\omega(\mathrm{p}-\mathrm{k})]-\cos 2 k_{0}\right)} .
\end{aligned}
$$

(b) $S e\left|\operatorname{Im} k_{0}\right|<m$, então:

$$
\int_{-\pi}^{\pi} R_{00}\left(\left(k_{0}, \mathrm{k}\right), p\right) d p_{0}=\frac{2 \pi Z(\mathrm{p}+\mathrm{k}) Z(\mathrm{p}-\mathrm{k}) \operatorname{senh}[\omega(\mathrm{p}+\mathrm{k})+\omega(\mathrm{p}-\mathrm{k})]}{\cosh [\omega(\mathrm{p}+\mathrm{k})+\omega(\mathrm{p}-\mathrm{k})]-\cos 2 k_{0}} .
$$

${ }^{21}$ Cf. [88], [89]. 
Seja agora $f \in L^{2}\left(T^{d}\right)$ com dependência apenas espacial. Então:

(c) A aplicação dada por:

$$
k_{0} \longmapsto \int_{T^{d+1}} R_{00}\left(\left(k_{0}, \mathrm{k}\right), p\right) f(\mathrm{p}) d p
$$

admite uma extensão analítica à região:

$$
\left\{\left|\operatorname{Re} k_{0}\right|<\pi \wedge\left|\operatorname{Im} k_{0}\right|<m+\delta_{0}\right\} \backslash\left\{\operatorname{Re} k_{0}=0 \wedge m \leqslant\left|\operatorname{Im} k_{0}\right|<m+\delta_{0}\right\} .
$$

(d) Suponha-se adicionalmente que $f$ toma apenas valores reais e, como na seção anterior, denotemos $\cos k_{0}-1=: x+i y$. Então, a distribuição definida em $\mathcal{D}(0,+\infty)$ por:

$$
h \longmapsto \lim _{y \rightarrow 0^{+}} \int_{0}^{\infty} \operatorname{Im}\left(\int_{T^{d+1}} R_{00}\left(\left(k_{0}, \mathrm{k}\right), p\right) f(\mathrm{p}) d p\right) h(x) d x
$$

é dada por:

$$
h \longmapsto \frac{\pi^{2}}{2} \int_{T^{d}} \frac{Z(\mathrm{p}+\mathrm{k}) Z(\mathrm{p}-\mathrm{k}) \operatorname{senh}[\omega(\mathrm{p}+\mathrm{k})+\omega(\mathrm{p}-\mathrm{k})] f(\mathrm{p}) h(a(\mathrm{p})-1)}{a(\mathrm{p})} d \mathrm{p},
$$

onde:

$$
a(\mathbf{p}):=\sqrt{\frac{\cosh [\omega(\mathbf{p}+\mathbf{k})+\omega(\mathbf{p}-\mathbf{k})]+1}{2}} .
$$

Demonstração: Por brevidade, denotemos:

$$
\begin{aligned}
\omega_{ \pm} & :=\omega(\mathbf{p} \pm \mathbf{k}) \\
a_{ \pm} & :=\cosh \omega_{ \pm}
\end{aligned}
$$

e também, como na seção anterior, $k_{0}=: x_{0}+i y_{0}$. Para a parte (a) temos:

$$
\begin{aligned}
& \int_{-\pi}^{\pi} \frac{1}{\cosh \omega(\mathrm{p}+\mathrm{k})-\cos \left(p_{0}+k_{0}\right)} \frac{1}{\cosh \omega(\mathrm{p}-\mathrm{k})-\cos \left(p_{0}-k_{0}\right)} d p_{0} \\
& \quad=\int_{|z|=1} \frac{1}{a_{+}-\frac{1}{2}\left(\mathrm{e}^{i k_{0}} z+\frac{\mathrm{e}^{-i k_{0}}}{z}\right)} \frac{1}{a_{-}-\frac{1}{2}\left(\mathrm{e}^{-i k_{0}} z+\frac{\mathrm{e}^{i k_{0}}}{z}\right)} \frac{d z}{i z}=\int_{|z|=1} F(z) d z,
\end{aligned}
$$

sendo $F$ a função dada por:

$$
F(z):=\frac{-4 i z}{\left(z-\alpha_{+}\right)\left(z-\alpha_{-}\right)\left(z-\beta_{+}\right)\left(z-\beta_{-}\right)},
$$


onde:

$$
\begin{aligned}
& \alpha_{ \pm}=\mathrm{e}^{ \pm \omega_{+}} \mathrm{e}^{-i k_{0}} \\
& \beta_{ \pm}=\mathrm{e}^{ \pm \omega_{-}} \mathrm{e}^{i k_{0}}
\end{aligned}
$$

Observe que $\left|\alpha_{ \pm}\right|=\mathrm{e}^{y_{0} \pm \omega_{+}}$e $\left|\beta_{ \pm}\right|=\mathrm{e}^{-y_{0} \pm \omega_{-}}$, portanto:

$$
\left|y_{0}\right|<m \Longrightarrow\left\{\begin{array} { l } 
{ | \alpha _ { + } | > 1 } \\
{ | \alpha _ { - } | < 1 }
\end{array} \wedge \left\{\begin{array}{l}
\left|\beta_{+}\right|>1 \\
\left|\beta_{-}\right|<1
\end{array}\right.\right.
$$

pois $\omega_{ \pm} \geqslant m$ e estamos supondo $\left|y_{0}\right|<m$. Assim, usando o Teorema dos Resíduos ${ }^{22}$ temos que a integral é dada por:

$$
\begin{aligned}
2 \pi i\left[\operatorname{Res}\left(F, \alpha_{-}\right)+\right. & \left.\operatorname{Res}\left(F, \beta_{-}\right)\right] \\
& =8 \pi \frac{\alpha_{-}\left(\beta_{-}-\alpha_{+}\right)\left(\beta_{-}-\beta_{+}\right)-\beta_{-}\left(\alpha_{-}-\alpha_{+}\right)\left(\alpha_{-}-\beta_{+}\right)}{\left(\alpha_{-}-\alpha_{+}\right)\left(\alpha_{-}-\beta_{+}\right)\left(\alpha_{-}-\beta_{-}\right)\left(\beta_{-}-\alpha_{+}\right)\left(\beta_{-}-\beta_{+}\right)} .
\end{aligned}
$$

Usando as definições, é simples verificar que:

$$
\begin{aligned}
\alpha_{-}-\alpha_{+} & =-2 \mathrm{e}^{-i k_{0}} \operatorname{senh} \omega_{+} \\
\beta_{-}-\beta_{+} & =-2 \mathrm{e}^{i k_{0}} \operatorname{senh} \omega_{-} \\
\alpha_{-}-\beta_{+} & =\mathrm{e}^{\omega_{-}}\left(\mathrm{e}^{-i k_{0}} \mathrm{e}^{-a}-\mathrm{e}^{i k_{0}}\right) \\
\beta_{-}-\alpha_{+} & =\mathrm{e}^{\omega_{+}}\left(\mathrm{e}^{i k_{0}} \mathrm{e}^{-a}-\mathrm{e}^{-i k_{0}}\right) \\
\alpha_{-}-\beta_{-} & =-2 i \mathrm{e}^{-\frac{a}{2}} \operatorname{sen}\left(k_{0}-i b / 2\right) \\
\left(\alpha_{-}-\beta_{+}\right)\left(\beta_{-}-\alpha_{+}\right) & =2\left(\cosh a-\cos 2 k_{0}\right),
\end{aligned}
$$

onde temos denotado $a:=\omega_{+}+\omega_{-}$e $b:=\omega_{+}-\omega_{-}$. Introduziendo as expressões acima na identidade anterior, um cálculo direto, porém laborioso, prova que a integral é dada por:

$$
\begin{aligned}
& 2 \pi \frac{\operatorname{senh} \omega_{+} \operatorname{sen}\left(k_{0}-i a / 2\right)+\operatorname{senh} \omega_{-} \operatorname{sen}\left(k_{0}+i a / 2\right)}{\operatorname{senh} \omega_{+} \operatorname{senh} \omega_{-} \operatorname{sen}\left(k_{0}-i b / 2\right)\left(\cosh a-\cos 2 k_{0}\right)} \\
& =2 \pi \frac{\operatorname{sen} k_{0} \cos (i a / 2)\left(\operatorname{senh} \omega_{+}+\operatorname{senh} \omega_{-}\right)+\cos k_{0} \operatorname{sen}(i a / 2)\left(\operatorname{senh} \omega_{+}-\operatorname{senh} \omega_{-}\right)}{\operatorname{senh} \omega_{+} \operatorname{senh} \omega_{-} \operatorname{sen}\left(k_{0}-i b / 2\right)\left(\cosh a-\cos 2 k_{0}\right)} .
\end{aligned}
$$

Daqui, a expressão do enunciado segue usando a identidade trigonométrica:

$$
\operatorname{senh} x+\operatorname{senh} y=2 \operatorname{senh}\left(\frac{x+y}{2}\right) \cosh \left(\frac{x-y}{2}\right)
$$

\footnotetext{
${ }^{22}$ Veja-se, v.g., [22] p. 108.
} 
para reescrever os fatores entre parênteses no numerador acima.

A parte (b) é conseqüência trivial de (a), e a parte (c) é conseqüência imediata de (b). Para a parte (d), observe que:

$$
\frac{1}{\cosh [\omega(\mathrm{p}+\mathrm{k})+\omega(\mathrm{p}-\mathrm{k})]-\cos 2 k_{0}}=\frac{1}{2} \frac{1}{a^{2}(\mathrm{p})-\cos ^{2} k_{0}} .
$$

Denotando agora $a:=a(\mathrm{p})$, para simplificar, um cálculo direto prova que:

$$
\begin{aligned}
\operatorname{Im}\left(\frac{1}{a^{2}-\cos ^{2} k_{0}}\right)=\frac{2(x+1) y}{\left[a^{2}-(x+1)^{2}+y^{2}\right]^{2}+4(x+1)^{2} y^{2}} \\
=\frac{2(x+1) y}{\left[a^{2}-(x+1)^{2}\right]^{2}+2 y^{2}\left[a^{2}+(x+1)^{2}\right]+y^{4}} .
\end{aligned}
$$

Portanto, usando os teoremas de Fubini e da convergência dominada e a parte (b), temos:

$$
\begin{aligned}
\lim _{y \rightarrow 0^{+}} \int_{0}^{\infty} \operatorname{Im}\left(\int_{T^{d+1}} R_{00}\left(\left(k_{0}, \mathrm{k}\right), p\right) f(\mathrm{p}) d p\right) h(x) d x \\
=\pi \int_{T^{d}} d \mathrm{p} Z(\mathrm{p}+\mathrm{k}) Z(\mathrm{p}-\mathrm{k}) \operatorname{senh}[\omega(\mathrm{p}+\mathrm{k})+\omega(\mathrm{p}-\mathrm{k})] f(\mathrm{p}) \\
\quad \times\left(\lim _{y \rightarrow 0^{+}} \int_{0}^{\infty} \frac{2(x+1) y}{\left[a^{2}-(x+1)^{2}\right]^{2}+2 y^{2}\left[a^{2}+(x+1)^{2}\right]+y^{4}} h(x) d x\right) .
\end{aligned}
$$

$\mathrm{O}$ resultado enunciado segue observando que o fator entre parênteses acima vale $\frac{\pi}{2 a} h(a-1)$, o que é provado no lema A.1 do apêndice ao final da presente seção.

O próximo passo consiste em generalizar o lema anterior para $R_{0}$. De (3.2.8), p. 42, temos:

$$
R_{0}(k, p)=2(2 \pi)^{\frac{d+1}{2}} \widehat{S}_{2}(k+p) \widehat{S}_{2}(k-p) .
$$

Usando a expressão em H1, p. 46, para a função de 2 pontos, tem-se que $R_{00}$ é (proporcional a) um dos somandos que aparecem em $R_{0}$. Introduzimos as seguintes definições:

$$
\begin{aligned}
\delta & :=\left(\delta_{0}, \delta_{1}, \ldots, \delta_{d}\right) \\
I_{\delta} & :=\left\{\left(\alpha_{0}, \alpha_{1}, \ldots, \alpha_{d}\right) \in \mathbb{R}^{d+1}:\left|\alpha_{i}\right| \leqslant \delta_{i} \forall i=0,1, \ldots, d\right\} \\
\|f\|_{\delta}^{2} & :=\sup _{\alpha \in I_{\delta}} \int_{T^{d+1}}|f(p+i \alpha)|^{2} d p
\end{aligned}
$$


$A_{\delta}:=\left\{f: f\right.$ é analítica na região $\left|\operatorname{Im} p_{i}\right| \leqslant \delta_{i},\|f\|_{\delta}<\infty$ e $\left.f(p)=f(-p)\right\}$.

Denotemos também com $D$ à região considerada em 3.4.1(c):

$D:=\left\{\left|\operatorname{Re} k_{0}\right|<\pi \wedge\left|\operatorname{Im} k_{0}\right|<m+\delta_{0}\right\} \backslash\left\{\operatorname{Re} k_{0}=0 \wedge m \leqslant\left|\operatorname{Im} k_{0}\right|<m+\delta_{0}\right\}$.

3.4.2 Lema: Sejam $g_{1}, g_{2} \in A_{\delta}$. Então:

(a) A aplicação dada por:

$$
k_{0} \longmapsto \int_{T^{d+1}} R_{0}\left(\left(k_{0}, 0\right), p\right) g_{1}(p) g_{2}(p) d p
$$

admite uma extensão analítica a $D$.

(b) Suponha-se adicionalmente que o produto $g_{1} g_{2}$ toma apenas valores reais e, como na seção anterior, denotemos $\cos k_{0}-1=: x+i y$. Então, a distribuição definida em $\mathcal{D}(0,+\infty)$ por:

$$
h \longmapsto \lim _{y \rightarrow 0^{+}} \int_{0}^{\infty} \operatorname{Im}\left(\int_{T^{d+1}} R_{0}\left(\left(k_{0}, 0\right), p\right) g_{1}(p) g_{2}(p) d p\right) h(x) d x
$$

é dada por:

$$
h \longmapsto 2(2 \pi)^{\frac{d+1}{2}} \pi^{2} \int_{T^{d}} Z^{2}(\mathbf{p}) \operatorname{senh} \omega(\mathbf{p}) g_{1}(0, \mathbf{p}) g_{2}(0, \mathbf{p}) h(\cosh \omega(\mathbf{p})-1) d \mathbf{p} .
$$

Demonstração: Denotemos:

$$
\begin{aligned}
R_{01} & :=R_{0}-2(2 \pi)^{\frac{d+1}{2}} R_{00} \\
f & :=g_{1} g_{2} \\
f_{1}(p) & :=f(p)-f(0, \mathbf{p}) .
\end{aligned}
$$

Com estas definições temos:

$$
\begin{aligned}
& \int_{T^{d+1}} R_{0}\left(\left(k_{0}, \mathrm{k}\right), p\right) g_{1}(p) g_{2}(p) d p \\
& \begin{aligned}
=2(2 \pi)^{\frac{d+1}{2}} \int_{T^{d+1}} R_{00}\left(\left(k_{0}, \mathrm{k}\right), p\right) f(0, \mathrm{p}) d p \\
+2(2 \pi)^{\frac{d+1}{2}} \int_{T^{d+1}} R_{00}\left(\left(k_{0}, \mathrm{k}\right), p\right) f_{1}(p) d p \\
\\
+\int_{T^{d+1}} R_{01}\left(\left(k_{0}, \mathrm{k}\right), p\right) f(p) d p .
\end{aligned}
\end{aligned}
$$


A idéia consiste o provar o resultado enunciado para cada um dos somandos no membro direito acima por separado. O caso do primeiro somando já foi provado em 3.4.1(c,d). Para tratar o segundo somando, como na prova de 3.4.1, denotemos $a_{ \pm}:=\cosh \omega(\mathrm{p} \pm \mathrm{k})$ e $k_{0}=: x_{0}+i y_{0}$. Consideremos primeiramente por um momento as funções $F_{+}$e $F_{-}$definidas por:

$$
F_{ \pm}(p):=\frac{1}{a_{ \pm}-\cos \left(p_{0} \pm k_{0}\right)}
$$

Se $p_{0}$ admite apenas valores reais, então:

$$
\cos \left(p_{0} \pm k_{0}\right)=\cos \left(p_{0} \pm x_{0}\right) \cosh y_{0} \mp i \operatorname{sen}\left(p_{0} \pm x_{0}\right) \operatorname{senh} y_{0}
$$

Assim, o denominador que aparece em $F_{ \pm}$será nulo se e somente se:

$$
\left\{\begin{array}{l}
\operatorname{sen}\left(p_{0} \pm x_{0}\right) \operatorname{senh} y_{0}=0 \\
\cos \left(p_{0} \pm x_{0}\right) \cosh y_{0}=\cosh \omega(\mathrm{p} \pm \mathrm{k})
\end{array}\right.
$$

Resulta simples verificar que das várias circunstâncias em que as duas identidades acima são satisfeitas, a única que efetivamente conduz a uma solução é:

$$
\left\{\begin{array}{l}
p_{0} \pm x_{0}=2 k \pi, \quad k \in \mathbb{Z} \\
y_{0}= \pm \omega(\mathrm{p} \pm \mathrm{k})
\end{array}\right.
$$

Aqui, os sinais duplos referem-se aos casos de $F_{+}$e $F_{-}$em separado, exceto o sinal duplo da segunda linha na frente da $\omega$ que vale para ambos casos (incidentalmente, observe que o único valor de $k \in \mathbb{Z}$ admitido em $D$ é o zero). Usando (3.4.1), podemos concluir que o segundo somando em (3.4.3) é analítico se $\left|y_{0}\right|<m$. Vamos supor então que $m \leqslant\left|y_{0}\right|<m+\delta_{0}$. Usando agora a expressão (3.4.2), a integral no segundo somando em (3.4.3) pode escrever-se como:

$$
\int_{T^{d+1}} \frac{Z^{2}(\mathrm{p}) \operatorname{senh}^{2}(\mathrm{p})}{-2 \operatorname{sen} k_{0}} \frac{f_{1}(p)}{\operatorname{sen} p_{0}} F_{+}(p) d p-\int_{T^{d+1}} \frac{Z^{2}(\mathrm{p}) \operatorname{senh}^{2}(\mathrm{p})}{-2 \operatorname{sen} k_{0}} \frac{f_{1}(p)}{\operatorname{sen} p_{0}} F_{-}(p) d p .
$$

Como antes, a idéia consiste em provar o enunciado para cada um destes novos somandos por separado. Para isto, a tal extensão analítica será definida construtivamente de maneira explícita. Seja portanto $k_{0}$ arbitrário mas fixo. Consideremos o primeiro dos somandos em (3.4.4). Observe que o primeiro fator do integrando é analítico como função de $k_{0}$ (pois estamos considerando que $m \leqslant\left|y_{0}\right|<m+\delta_{0}$ ), e que o segundo fator não tem singularidades como função de $p_{0}$ (pois as propriedades de analiticidade da $f_{1}$ levantam a singularidade do seno no denominador). Temos assim que as dificuldades que conspiram contra a analiticidade provêm apenas de 
$F_{+}$. Admitindo desta vez que $p_{0}$ assume valores complexos, e denotando $p_{0}=p_{0}+i \eta$, temos, como antes, que o denominador em $F_{+}$é nulo se e somente se:

$$
\left\{\begin{array}{l}
p_{0}+x_{0}=2 k \pi, \quad k \in \mathbb{Z}, \\
\eta+y_{0}= \pm \omega(\mathrm{p}+\mathrm{k}) .
\end{array}\right.
$$

Para fixar idéias, vamos supor que $m \leqslant y_{0}<m+\delta_{0}$. Observe-se que escolhendo:

$$
\eta=-\left(y_{0}-m+\epsilon\right)
$$

com $0<\epsilon<\min \left\{m+\delta_{0}-y_{0}, 2 m\right\}$, então a segunda identidade acima não pode ser satisfeita (pois $m \leqslant \omega(\mathrm{p}+\mathrm{k})$ ). Assim, a idéia consiste em deslocar o contorno da integração em $p_{0}$ para $p_{0}+i \eta$ e depois fazer $\eta$ tender para zero. Observe que de todas as possíveis escolhas de $\eta$ que servem a este propósito, a nossa garante que $|\eta|<\delta_{0}$, estando portanto $p_{0}+i \eta$ na região de analiticidade das funções de $A_{\delta}$. De fato, nisto radica a limitação do método, pois para garantir que $|\eta|<\delta_{0}$ é preciso que $y_{0}<m+\delta_{0}-\epsilon$, ou como $\epsilon$ pode ser arbitrariamente pequeno, que $y_{0}<m+\delta_{0}$. $\mathrm{O}$ caso de $-\left(m+\delta_{0}\right)<y_{0} \leqslant-m$ é análogo (deve escolher-se $\left.\eta=-\left(y_{0}+m-\epsilon\right)\right)$. $\mathrm{O}$ caso do segundo somando em (3.4.4) é análogo ao caso do primeiro. Isto prova o item (a) para o segundo somando em (3.4.3). Provaremos a continuação que a sua contribuição para a parte (b) é nula. Denotando $a:=\cosh \omega(\mathbf{p})$, um cálculo direto e manipulações algébricas provam que:

$$
\begin{aligned}
& \operatorname{Im}\left(\frac{1}{a-\cos \left(p_{0}+k_{0}\right)} \frac{1}{a-\cos \left(p_{0}-k_{0}\right)}\right) \\
& \quad=\frac{-2 y\left[(x+1)-a \cos p_{0}\right]}{\left(\left[(x+1)-a \cos p_{0}\right]^{2}+\left(a^{2}-1\right) \operatorname{sen}^{2} p_{0}-y^{2}\right)^{2}+4 y^{2}\left[(x+1)-a \cos p_{0}\right]^{2}} .
\end{aligned}
$$

Assim, de (3.4.1) e usando os teoremas de Fubini e da convergência dominada, temos:

$$
\begin{aligned}
& \lim _{y \rightarrow 0^{+}} \int_{0}^{\infty} \operatorname{Im}\left(\int_{T^{d+1}} R_{00}\left(\left(k_{0}, 0\right), p\right) f_{1}(p) d p\right) h(x) d x \\
& =\int_{T^{d}} d \mathrm{p} Z^{2}(\mathrm{p}) \operatorname{senh}^{2} \omega(\mathrm{p}) \\
& \times\left(\lim _{y \rightarrow 0^{+}} \int_{-\pi}^{\pi} \int_{0}^{\infty} \frac{-2 y\left[(x+1)-a \cos p_{0}\right] h(x) f_{1}\left(p_{0}, \mathrm{p}\right) d x d p_{0}}{\left(\left[(x+1)-a \cos p_{0}\right]^{2}+\left(a^{2}-1\right) \operatorname{sen}^{2} p_{0}-y^{2}\right)^{2}+4 y^{2}\left[(x+1)-a \cos p_{0}\right]^{2}}\right) .
\end{aligned}
$$

No lema A.2 do apêndice ao final desta seção é provado que o fator entre parênteses acima é proporcional a $h(a-1) f_{1}(0, \mathrm{p})$, que é nulo, pela definição de $f_{1}$.

Finalmente, o caso do terceiro somando em (3.4.3) é análogo ao caso do segundo, sendo a sua contribuição para a parte (b) também nula (Cf. [101] p. 8). 
3.4.3 Observação: Da prova de 3.4.2(a) segue que a aplicação:

$$
k_{0} \longmapsto \int_{T^{d+1}} R_{0}\left(\left(k_{0}, 0\right), p\right) f_{1}(p) d p
$$

é limitada em $D$.

\subsubsection{Observações:}

(a) A norma dada por:

$$
\|f\|_{\delta^{\prime}}^{2}:=\sum_{x \in \mathbb{Z}^{d+1}} \mathrm{e}^{2 \sum_{i=0}^{d} \delta_{i}\left|x_{i}\right|}|\check{f}(x)|^{2}
$$

é equivalente a $\|\cdot\|_{\delta}$. Com efeito, de $f(p+i \alpha)=\left(\mathrm{e}^{\alpha x} \check{f}(x)\right)^{\wedge}(p)$, usando a identidade de Plancherel temos:

$$
\int|f(p+i \alpha)|^{2} d p=\sum_{x} \mathrm{e}^{2 \alpha x}|\check{f}(x)|^{2} \leqslant \sum_{x} \mathrm{e}^{2 \delta|x|}|\check{f}(x)|^{2} \quad \forall|\alpha| \leqslant \delta,
$$

de onde segue que $\|f\|_{\delta} \leqslant\|f\|_{\delta^{\prime}}$. Por outro lado:

$$
\begin{aligned}
\sum_{x \geqslant 0} \mathrm{e}^{2 \alpha x}|\check{f}(x)|^{2} \leqslant \sum_{x} \mathrm{e}^{2 \alpha x}|\check{f}(x)|^{2}=\int|f(p+i \alpha)|^{2} d p & \\
& \leqslant \sup _{|\alpha| \leqslant \delta} \int|f(p+i \alpha)|^{2} d p=\|f\|_{\delta}^{2} \quad \forall|\alpha| \leqslant \delta .
\end{aligned}
$$

Em particular, para $\alpha=\delta$ temos:

$$
\sum_{x \geqslant 0} \mathrm{e}^{2 \delta x}|\check{f}(x)|^{2} \leqslant\|f\|_{\delta}^{2} .
$$

Analogamente (tomando desta vez $\alpha=-\delta$ ) tem-se:

$$
\sum_{x<0} \mathrm{e}^{-2 \delta x}|\check{f}(x)|^{2} \leqslant\|f\|_{\delta}^{2}
$$

Somando as últimas duas identidades segue que $\|f\|_{\delta^{\prime}}^{2} \leqslant 2\|f\|_{\delta}^{2}$. Aqui, para simplificar a notação temos provado o caso unidimensional. A generalizacão para dimensão $d+1$ é imediata e análoga. Em virtude desta equivalência e para evitar ambigüidades denotaremos:

$$
\begin{aligned}
A_{\delta} & :=\left(A_{\delta},\|\cdot\|_{\delta}\right) \\
A_{\delta^{\prime}} & :=\left(A_{\delta},\|\cdot\|_{\delta^{\prime}}\right) .
\end{aligned}
$$


No seguinte vamos supor que $\delta_{i}=m-\epsilon \forall i=0,1, \ldots, d$, onde $\epsilon>0$ é arbitráriamente pequeno (não confundir com o $\epsilon$ da prova de 3.4.2).

(b) A aplicação $q \longmapsto K(k, p, q)$ pertence a $A_{\delta^{\prime}}$, e pelo item (a) anterior, também a $A_{\delta}$. Com efeito, $\check{K}$ decai exponencialmente com taxa $m$ e portanto $\|K\|_{\delta^{\prime}}^{2}$ é basicamente a soma de uma série geométrica de razão $e^{-2 \epsilon}$.

(c) Pelo item (a) anterior, a aplicação $U: f \longmapsto \mathrm{e}^{\sum_{i=0}^{d} \delta_{i}\left|x_{i}\right|} \check{f}(x)$ é um operador unitário de $A_{\delta^{\prime}}$ em $\ell^{2}\left(\mathbb{Z}^{d+1}\right)$. Resulta imediato verificar que:

$$
U^{-1} f(p)=(2 \pi)^{-\frac{d+1}{2}} \sum_{x \in \mathbb{Z}^{d+1}} \mathrm{e}^{-i p \cdot x} \mathrm{e}^{-\sum_{i=0}^{d} \delta_{i}\left|x_{i}\right|} f(x) .
$$

(d) Usando o item (c) acima, resulta simples verificar que o operador $U K(k) R_{0}(k) U^{-1}$ é dado pelo núcleo "integral":

$$
\begin{aligned}
& F(x, y) \\
& =\frac{1}{(2 \pi)^{d+1}} \int_{T^{d+1}} \int_{T^{d+1}} \mathrm{e}^{\sum_{i=0}^{d} \delta_{i}\left|x_{i}\right|} \mathrm{e}^{i x \cdot p} K(k, p, q) R_{0}(k, q) \mathrm{e}^{-i q \cdot y} \mathrm{e}^{-\sum_{i=0}^{d} \delta_{i}\left|y_{i}\right|} d q d p .
\end{aligned}
$$

\subsubsection{Lema:}

(a) $K\left(k_{0}, 0\right) R_{0}\left(k_{0}, 0\right)$ é uma familia analítica na região $D$ de operadores em $A_{\delta}$.

(b) Os operadores da família analítica do ponto (a) restritos à região:

$D_{1}:=\left\{\left|\operatorname{Re} k_{0}\right|<\pi / 2 \wedge\left|\operatorname{Im} k_{0}\right|<m+\delta_{0}\right\} \backslash\left\{\operatorname{Re} k_{0}=0 \wedge m \leqslant\left|\operatorname{Im} k_{0}\right|<m+\delta_{0}\right\}$.

são de Hilbert-Schmidt. Em particular, são compactos em $D_{1}$.

(c) $\left[I+K\left(k_{0}, 0\right) R_{0}\left(k_{0}, 0\right)\right]^{-1}$ existe (e é analítico) em $D_{1}$ salvo um conjunto discreto $S \subset D_{1}$.

Demonstração: A parte (a) segue de 3.4.2(a) (pois $K \in A_{\delta}$, segundo 3.4.4(b)). Para a parte (b), observe que, por 3.4.4(d), o quadrado da norma de Hilbert-Schmidt de $U K(k) R_{0}(k) U^{-1}$ é dado por:

$$
\begin{aligned}
& (2 \pi)^{2(d+1)}\left\|U K(k) R_{0}(k) U^{-1}\right\|_{H-S}^{2}=(2 \pi)^{2(d+1)} \sum_{x, y \in \mathbb{Z}^{d+1}}|F(x, y)|^{2} \\
& =\sum_{x, y \in \mathbb{Z}^{d+1}}\left|\iint \mathrm{e}^{\sum_{i=0}^{d} \delta_{i}\left|x_{i}\right|} \mathrm{e}^{i x . p} K(k, p, q) R_{0}(k, q) \mathrm{e}^{-i q \cdot y} \mathrm{e}^{-\sum_{i=0}^{d} \delta_{i}\left|y_{i}\right|} d q d p\right|^{2} .
\end{aligned}
$$


Que isto é finito segue observando, primeiramente, que a integral em $d p$ é a antitransformada de Fourier de $K$ na sua segunda variável, a qual decai exponencialmente com taxa $m$, o que controla o primeiro fator exponencial no integrando, assegurando assim a convergência da somatória em $x$. A integral em $d q$ é uma integral do tipo que aparece em 3.4.2(a), a qual é finita pois a função no integrando é limitada, dado que, fixado $k_{0} \in D_{1}$, o denominador no membro direito de 3.4.1(b) tem módulo maior o igual que $\left|\cosh ^{2} m-\cosh ^{2} y_{0}\right|>0$ se $0<\left|y_{0}\right|<m$, ou que $2\left|\cos x_{0} \operatorname{sen} x_{0} \operatorname{senh} y_{0} \cosh y_{0}\right|>0$ se $\left|x_{0}\right|<\pi / 2$, no caso $m \leqslant\left|y_{0}\right|<m+\delta_{0}$. Assim, a convergência da somatória em $y$ segue do decaimento exponencial do último fator no integrando acima. A parte (c) é um fato bem conhecido sobre operadores compactos (veja-se Lemma 13 em [27] p. 592; Cf. teoremas 3.3 e 3.4 em [101] p. 10)

\section{Apêndice à Seção 3.4: Detalhes Técnicos}

Neste apêndice apresentamos as provas de certos resultados usados nas demonstrações dos lemas da presente seção. ${ }^{23}$

A.1 Lema: Seja $h \in \mathcal{D}(\mathbb{R})$ com $\operatorname{supp} h \subset(0,+\infty)$, e $a>0$. Então:

$$
\lim _{y \rightarrow 0^{+}} \int_{0}^{\infty} \frac{2 x y}{\left(a^{2}-x^{2}\right)^{2}+2 y^{2}\left(a^{2}+x^{2}\right)+y^{4}} h(x) d x=\frac{\pi}{2 a} h(a) .
$$

Demonstração: Com repetidas mudanças de variáveis nas integrais e manipulações algébricas no primeiro fator do integrando, temos:

$$
\begin{gathered}
\int_{0}^{\infty} \frac{2 x y}{\left(a^{2}-x^{2}\right)^{2}+2 y^{2}\left(a^{2}+x^{2}\right)+y^{4}} h(x) d x \\
=\int_{0}^{\infty} \frac{y}{\left(a^{2}-u\right)^{2}+2 y^{2}\left(a^{2}+u\right)+y^{4}} h(\sqrt{u}) d u \\
=\int_{0}^{\infty} \frac{y}{4 y^{2} a^{2}\left[\left(\frac{u-\left(a^{2}-y^{2}\right)}{2 y a}\right)^{2}+1\right]} h(\sqrt{u}) d u \\
=\frac{1}{2 a} \int_{-\frac{a^{2}-y^{2}}{2 y a}}^{\infty} \frac{1}{v^{2}+1} h\left(\sqrt{2 a y v+a^{2}-y^{2}}\right) d v .
\end{gathered}
$$

\footnotetext{
${ }^{23}$ Cf. Lemma 2.4 .2 em [63] p. 127.
} 
Denotando $h_{1}(v):=h(v)-h(a)$, a integral da última linha acima pode escrever-se como:

$$
h(a) \int_{-\frac{a^{2}-y^{2}}{2 y a}}^{\infty} \frac{1}{v^{2}+1} d v+\int_{-\frac{a^{2}-y^{2}}{2 y a}}^{\infty} \frac{1}{v^{2}+1} h_{1}\left(\sqrt{2 a y v+a^{2}-y^{2}}\right) d v .
$$

Observe que na integral no primeiro somando o limite inferior de integração converge para $-\infty$ se $y \rightarrow 0^{+}$. Assim, a integral converge para uma integral em $(-\infty,+\infty)$, cujo valor é $\pi$. Basta portanto provar que o segundo somando converge para zero quando $y$ faz o mesmo. Para isto, observe que:

$$
\left|\int_{-\frac{a^{2}-y^{2}}{2 y a}}^{\infty} \frac{1}{v^{2}+1} h_{1}\left(\sqrt{2 a y v+a^{2}-y^{2}}\right) d v\right| \leqslant \pi \sup _{v \in \mathbb{R}}\left|h_{1}\left(\sqrt{2 a y v+a^{2}-y^{2}}\right)\right| .
$$

Agora, como $h_{1}$ é contínua, tem-se:

$$
\lim _{y \rightarrow 0^{+}} h_{1}\left(\sqrt{2 a y v+a^{2}-y^{2}}\right)=h_{1}(a)=0,
$$

sendo este último limite uniforme em $v$ pela compacidade do suporte de $h_{1}$, e disto segue o afirmado.

A.2 Lema: Seja $F \in \mathcal{D}\left(\mathbb{R}^{2}\right)$ com $\operatorname{supp} F \subset(0,+\infty) \times \mathbb{R}$, e a $>1$. Então:

$$
\begin{array}{r}
\lim _{y \rightarrow 0^{+}} \int_{-\pi}^{\pi} \int_{0}^{\infty} \frac{-2 y\left(x-a \cos p_{0}\right) F\left(x, p_{0}\right) d x d p_{0}}{\left[\left(x-a \cos p_{0}\right)^{2}+\left(a^{2}-1\right) \operatorname{sen}^{2} p_{0}-y^{2}\right]^{2}+4 y^{2}\left(x-a \cos p_{0}\right)^{2}} \\
=\text { const } \frac{F(a, 0)}{\sqrt{a^{2}-1}}
\end{array}
$$

Demonstração: Primeiramente, observe que

$$
\frac{-2 y\left(x-a \cos p_{0}\right)}{\left[\left(x-a \cos p_{0}\right)^{2}+\left(a^{2}-1\right) \operatorname{sen}^{2} p_{0}-y^{2}\right]^{2}+4 y^{2}\left(x-a \cos p_{0}\right)^{2}}
$$

converge para zero uniformemente em compactos de $(0,+\infty) \times(\mathbb{R} \backslash 0)$. Com efeito, quando $y \longrightarrow 0^{+}$o numerador da expressão acima converge para zero uniformemente em compactos como função de $x$ e uniformemente como função de $p_{0}$, ao igual que o segundo somando do denominador. O primeiro somando do denominador converge uniformemente sobre compactos como função de $x$ e uniformemente como função de $p_{0}$ para uma função nunca nula quando $p_{0} \neq 0$ (observe que também deveriamos 
excluir os valores $p_{0}= \pm \pi$, mas isto é desnecessário se $x>0$ ). Se na integral do enunciado, dividemos a integral en $d p_{0}$ em duas partes, uma com $\left|p_{0}\right| \geqslant \pi / 2$ e a outra com $\left|p_{0}\right|<\pi / 2$, pela observação acima, temos que a primeira integral converge para zero quando $y$ faz o próprio. Para tratar a segunda integral, consideremos primeiramente a aplicação $g: \mathbb{R}^{2} \longmapsto \mathbb{R}^{2}$ dada por:

$$
g\left(x, p_{0}\right):=\left(x-a \cos p_{0}, \sqrt{a^{2}-1} \operatorname{sen} p_{0}\right)=:(u, v) .
$$

Observe-se que o determinante jacobiano de $g$ vale $\sqrt{a^{2}-1} \cos p_{0}$ que é não nulo se $\left|p_{0}\right|<\pi / 2$. Usando a mudança de variáveis determinada por $g$, temos então:

$$
\begin{aligned}
\int_{-\pi / 2}^{\pi / 2} \int_{0}^{\infty} \frac{-2 y\left(x-a \cos p_{0}\right) F\left(x, p_{0}\right) d x d p_{0}}{\left[\left(x-a \cos p_{0}\right)^{2}+\left(a^{2}-1\right) \operatorname{sen}^{2} p_{0}-y^{2}\right]^{2}+4 y^{2}\left(x-a \cos p_{0}\right)^{2}} \\
=\int_{-a}^{\infty} \int_{-\sqrt{a^{2}-1}}^{+\sqrt{a^{2}-1}} \frac{-2 y u}{\left(u^{2}+v^{2}-y^{2}\right)^{2}+4 y^{2} u^{2}} G(u, v) d u d v \\
=\int_{-\frac{a}{y}}^{\infty} \int_{-\frac{\sqrt{a^{2}-1}}{y}}^{+\frac{\sqrt{a^{2}-1}}{y}} \frac{-2 \alpha}{\left(\alpha^{2}+\beta^{2}-1\right)^{2}+4 \alpha^{2}} G(y \alpha, y \beta) d \alpha d \beta .
\end{aligned}
$$

Aqui, $G$ denota a função dada por:

$$
G(u, v):=\frac{F\left(g^{-1}(u, v)\right)}{\sqrt{a^{2}-1-v^{2}}}
$$

e para a última igualdade usamos a mudança de variáveis dada por:

$$
(\alpha, \beta):=(u / y, v / y) \text {. }
$$

Como no lema anterior, denotando $G_{1}(u, v):=G(u, v)-G(0,0)$, esta última integral vale:

$$
\text { const } G(0,0)+\int_{-\frac{a}{y}}^{\infty} \int_{-\frac{\sqrt{a^{2}-1}}{y}}^{+\frac{\sqrt{a^{2}-1}}{y}} \frac{-2 \alpha}{\left(\alpha^{2}+\beta^{2}-1\right)^{2}+4 \alpha^{2}} G_{1}(y \alpha, y \beta) d \alpha d \beta .
$$

Também como na prova do lema anterior, o módulo do segundo somando é majorado por:

$$
\text { const } \sup _{(\alpha, \beta) \in \mathbb{R}^{2}}\left|G_{1}(y \alpha, y \beta)\right| \text {. }
$$

Agora, $G_{1}(y \alpha, y \beta)$ tende para $G_{1}(0,0)=0$ quando $y \rightarrow 0$, pela continuidade de $G_{1}$ e este limite é uniforme pela compacidade do seu suporte. Finalmente, observando que $g(a, 0)=(0,0)$, de onde $g^{-1}(0,0)=(a, 0)$, temos que o limite do enunciado vale:

$$
\text { const } G(0,0)=\text { const } \frac{F\left(g^{-1}(0,0)\right)}{\sqrt{a^{2}-1}}=\text { const } \frac{F(a, 0)}{\sqrt{a^{2}-1}} \text {. }
$$




\subsection{O Espectro de Energia}

O conjunto discreto $S$ em 3.4.5(c) será denominado o conjunto de polos de $I+K\left(k_{0}, 0\right) R_{0}\left(k_{0}, 0\right)$ e todo operador que, como este, satisfaça 3.4.5(c) será dito meromorfo em $D_{1}$. Assim, pelo lema 3.4.5(c), temos que $R\left(k_{0}, 0\right)$ existe e é meromorfo em $D_{1}$, estando dado por:

$$
R\left(k_{0}, 0\right)=R_{0}\left(k_{0}, 0\right)\left[I+K\left(k_{0}, 0\right) R_{0}\left(k_{0}, 0\right)\right]^{-1} .
$$

No seguinte, o conjunto discreto de polos de $R\left(k_{0}, 0\right)$ será denotado por $S$.

3.5.1 Lema: Seja $f \in A_{\delta}$. Denotemos $\cos k_{0}-1=x+i y$.

(a) Seja $(\alpha, \beta) \subseteq(0, m)$ com $\alpha>0$ e suponha-se que $R\left(k_{0}, 0\right)$ não tem polos em $\left\{k_{0}=i y_{0}: y_{0} \in(\alpha, \beta)\right\}$. Então a distribuição dada por:

$$
h \longmapsto \lim _{y \rightarrow 0^{+}} \int \operatorname{Im}\left\langle f, R\left(k_{0}, 0\right) f\right\rangle h(x) d x
$$

está bem definida em $\mathcal{D}(\cosh \alpha-1, \cosh \beta-1)$ e é nula.

(b) Seja $0<\delta_{1} \leqslant \delta_{0}$ e suponha-se que não existem pontos de acumulação de polos em $\left\{k_{0}=i y_{0}: m \leqslant y_{0}<m+\delta_{1}\right\}$. Então, se $(\alpha, \beta) \subseteq\left(0, m+\delta_{1}\right)$ com $\alpha>0, a$ distribuição acima está bem definida em $\mathcal{D}(\cosh \alpha-1, \cosh \beta-1)$, sendo nula se $\beta \leqslant m$ ou, caso contrário, estando dada por:

$h \longmapsto 2(2 \pi)^{\frac{d+1}{2}} \pi^{2} \int_{T^{d}} Z^{2}(\mathbf{p}) \operatorname{senh} \omega(\mathbf{p}) W f(0, \mathbf{p}) \overline{W f}(0, \mathbf{p}) h(\cosh \omega(\mathbf{p})-1) d \mathbf{p}$,

onde:

$$
W f:=\lim _{\substack{y \rightarrow 0^{+} \\ x \rightarrow \cosh \omega(\mathbf{p})-1}}\left[I+K\left(k_{0}, 0\right) R_{0}\left(k_{0}, 0\right)\right]^{-1} f .
$$

Demonstração: Para a parte (a), observe que:

$$
\begin{aligned}
& x=\cosh \left(y_{0}\right) \cos \left(x_{0}\right)-1 \\
& y=-\operatorname{sen}\left(x_{0}\right) \operatorname{senh}\left(y_{0}\right) .
\end{aligned}
$$

Portanto, se $y_{0} \in(\alpha, \beta)$ com $\alpha>0$ então $y \longrightarrow 0^{+}$se e somente se $x_{0} \longrightarrow 0^{-}$. Por outro lado, $\forall \epsilon>0 \exists \delta>0:-\delta<x_{0}<0 \Longrightarrow x \in(\cosh \alpha-1+\epsilon, \cosh \beta-1-\epsilon)$. Disso segue a primeira afirmação. A prova da segunda é como a de 3.4.1(d) (Cf. 
A.1 no apêndice à seção 3.4 ).

Para a parte (b), da prova do Lemma 5.3 que consta em [101] p. 14, temos que:

$$
\begin{aligned}
\int \operatorname{Im}\left\langle f, R\left(k_{0}, 0\right) f\right\rangle h(x) d x & =\int \operatorname{Im}\left\langle g, R_{0}\left(k_{0}, 0\right) g\right\rangle h(x) d x \\
= & \int \operatorname{Im}\left(\int_{T^{d+1}} R_{0}\left(\left(k_{0}, 0\right), p\right) g(p) \overline{g(p)} d p\right) h(x) d x .
\end{aligned}
$$

onde $g:=\left[I+K\left(k_{0}, 0\right) R_{0}\left(k_{0}, 0\right)\right]^{-1} f$. O resto é como na prova de 3.4.1(d) (mais especificamente, Cf. a prova de A.1 no apêndice à seção 3.4).

O próximo resultado tem certa relevância e para enunciá-lo introduzimos a seguinte notação, que será usada também nas próximas seções: se $A \subset(0,+\infty) \times$ $(\pi, \pi]^{d}$ é um conjunto Boreliano da forma $A=(0, a) \times 0$ e $E$ denota a família espectral do operador de energia-momento $(H, \mathbf{P})$, denotamos:

$$
\mathcal{H}^{a}:=E(A) \mathcal{H}
$$

Denotemos também por $A_{\delta}^{1}$ o subespaço de $A_{\delta}$ formado pelas funções com dependência apenas espacial.

3.5.2 Lema: Denotemos por $\sigma$ o espectro do operador de energia-momento restrito ao subespaço:

$$
\overline{\operatorname{span}}\left\{\Theta(\check{f}): f \in A_{\delta}^{1}\right\} \cap \mathcal{H}^{2\left(m+\delta_{0}\right)} .
$$

Então:

$$
(0,2 m) \cap \sigma \subseteq-2 i\left[S \cap\left\{k_{0}=i y_{0}: y_{0} \in(0, m)\right\}\right]
$$

Demonstração: Basta provar o lema em $(\epsilon, 2 m-\epsilon)$ para $\epsilon>0$ arbitrariamente pequeno. Por reductio ad absurdum, suponha-se a existência de $\lambda \in(\epsilon, 2 m-\epsilon) \cap \sigma$ mas que $i \lambda / 2 \notin S$. Em tal caso, como $S$ é discreto em $D_{1}$, existe uma vizinhança de $i \lambda / 2$ que não contém pontos de $S$, ou seja, polos. Denotando $(\alpha, \beta)$ a interseção desta vizinhança com o eixo imaginário, por 3.5.1(a) e 3.3.1 a medida espectral correspondente é nula em $(2 \alpha, 2 \beta) \ni \lambda$, o qual constitui uma contradição.

\subsection{Ausência de Estados Ligados}

O passo final consiste em eliminar a possibilidade de estados ligados. Para isto é necessário a introdução da seguinte hipótese: 
H3 $K$ está dado como núcleo integral por:

$$
K(k, p, q)=a 1+a^{2} K_{1}(k, p, q)
$$

com $K_{1}$ satisfazendo a hipótese H2, p. 46. Aqui 1 denota a função identicamente igual a 1 e $a$ é uma constante positiva.

Esta hipótese é uma paráfrase da condição $\left(^{* *}\right)$ no trabalho de Spencer e Zirilli, [101] p. 5. Foi verificada por Spencer, [99], para certos modelos $P(\varphi)_{2}$ com acoplamento fraco. Aliás, esta característica é específica de tais interações. Assim, a generalidade dos resultados da presente seção fica mais comprometida.

Sob a hipótese $\mathrm{H} 3$ resulta simples verificar que o $R$ está dado por:

$$
R=R_{0}^{\prime}\left(I+a^{2} K_{1} R_{0}^{\prime}\right)^{-1}
$$

onde:

$$
R_{0}^{\prime}:=\left(R_{0}^{-1}+a 1\right)^{-1} .
$$

Também é simples verificar ${ }^{24}$ que $R_{0}^{\prime}$ é dado como núcleo integral por:

$$
R_{0}^{\prime}(k, p, q)=R_{0}(k, p) \delta(p-q)-\frac{a}{a r_{0}(k)+1} R_{0}(k, p) R_{0}(k, q)
$$

onde $r_{0}(k):=\int R_{0}(k, p) d p$. Como $K_{1}$ satisfaz a hipótese $\mathrm{H} 2$ e $R_{0}^{\prime}$ é basicamente como $R_{0}$, a análise é totalmente análoga à da seção 3.4. Para provar que $R$ não tem polos, basta provar que a norma de $a^{2} K_{1} R_{0}^{\prime}$ é pequena, e para isto é suficiente que a sua norma de Hilbert-Schmidt $\left\|a^{2} K_{1} R_{0}^{\prime}\right\|_{H-S}$ o seja.

3.6.1 Lema: Se a constante a de H3 é suficientemente pequena, então existe $\mu>0$ tal que o operador $\left[I+a^{2} K_{1}\left(k_{0}, 0\right) R_{0}^{\prime}\left(k_{0}, 0\right)\right]^{-1}$ não tem polos em:

$$
\left[D_{1} \cap\left\{k_{0}: 0<\left|\operatorname{Im} k_{0}\right|<m\right\}\right] \cup\left\{k_{0}:\left|k_{0}-i m\right|<\mu\right\} .
$$

Ou seja, a mesma constante a serve para todos os $k_{0}$ uniformemente na região acima. Mais ainda, isto permanece válido mesmo no limite $x_{0} \longrightarrow 0$.

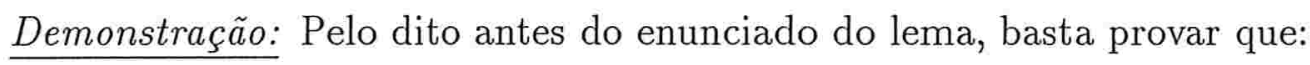

$$
\left\|K_{1}\left(k_{0}, 0\right) R_{0}^{\prime}\left(k_{0}, 0\right)\right\|_{H-S} \leqslant c(k)+o\left(a^{-1}\right),
$$

\footnotetext{
${ }^{24}$ Veja-se [101] p. 11, Lemma 4.1.
} 
onde $c(k)$ é limitada e $o\left(a^{-1}\right)$ é uma magnitude da ordem de $1 / a$, ambas ainda no limite $x_{0} \longrightarrow 0$. Usando (3.6.1) e 3.4.4(c), resulta simples verificar que o operador $U K_{1} R_{0}^{\prime} U^{-1}$ é dado pelo núcleo "integral":

$$
\begin{aligned}
F(x, y) & =\frac{1}{(2 \pi)^{d+1}} \iint \mathrm{e}^{\sum_{i=0}^{d} \delta_{i}\left|x_{i}\right|} \mathrm{e}^{i x \cdot p} K_{1}(k, p, q) \mathrm{e}^{-\sum_{i=0}^{d} \delta_{i}\left|y_{i}\right|} \\
\times & {\left[R_{0}(k, q) \mathrm{e}^{-i q \cdot y}-\frac{a}{a r_{0}(k)+1}\left(\int R_{0}(k, r) \mathrm{e}^{-i r \cdot y} d r\right) R_{0}(k, q)\right] d q d p . }
\end{aligned}
$$

Denotando:

$$
f_{x, y}(q):=\frac{1}{(2 \pi)^{d+1}} \int \mathrm{e}^{\sum_{i=0}^{d} \delta_{i}\left|x_{i}\right|} \mathrm{e}^{i x \cdot p} K_{1}(k, p, q) \mathrm{e}^{-\sum_{i=0}^{d} \delta_{i}\left|y_{i}\right|} d p,
$$

temos:

$$
\begin{aligned}
& F(x, y)=\int R_{0}(k, q) \mathrm{e}^{-i q \cdot y} f_{x, y}(q) d q \\
& -\frac{a}{a r_{0}(k)+1}\left(\int R_{0}(k, r) \mathrm{e}^{-i r \cdot y} d r\right) \int R_{0}(k, q) f_{x, y}(q) d q \\
& =\frac{1}{a r_{0}(k)+1}\left[a r_{0}(k) \int R_{0}(k, q) \mathrm{e}^{-i q \cdot y} f_{x, y}(q) d q+\int R_{0}(k, q) \mathrm{e}^{-i q \cdot y} f_{x, y}(q) d q\right. \\
& \left.-a\left(\int R_{0}(k, r) \mathrm{e}^{-i r \cdot y} d r\right) \int R_{0}(k, q) f_{x, y}(q) d q\right] \cdot
\end{aligned}
$$

No seguinte vamos denotar $f_{x, y}(q)=: f(q)$ para simplificar. Observe que quando $f$ é constante como função de $q$, o primeiro e terceiro somandos entre colchetes acima se cancelam, resultando (3.6.2) proporcional a:

$$
\frac{\int R_{0}(k, q) \mathrm{e}^{-i q \cdot y} d q}{a r_{0}(k)+1} .
$$

Agora, observe que:

$$
\left|\frac{\int R_{0}(k, q) \mathrm{e}^{-i q \cdot y} d q}{a r_{0}(k)+1}\right| \leqslant \frac{\int\left|R_{0}(k, q)\right| d q}{\left|a r_{0}(k)+1\right|} \leqslant \frac{\rho_{0}}{1-a \rho_{0}}=\frac{1}{\rho_{0}^{-1}-a},
$$

onde temos denotado $\rho_{0}:=\int\left|R_{0}(k, q)\right| d q$. Observe que esta última expressão é da ordem de $1 / a$, ainda no limite $r_{0}(k) \rightarrow \infty$. Ou seja, denotando:

$$
\begin{aligned}
& f_{1}(q):=f(q)-f(0, \mathbf{q}) \\
& f_{2}(\mathbf{q}):=f(0, \mathbf{q})-f(0),
\end{aligned}
$$


temos que $f(q)=f(0)+f_{1}(q)+f_{2}(\mathrm{q})$ e, pelo antevisto, para provar o lema basta provar a seguinte

Afirmação: Para cada $i=1,2$, a expressão:

$$
\int R_{0}(k, q) f_{i}(q) d q
$$

é limitada como função de $k_{0}$ na região do enunciado, mesmo no limite $x_{0} \rightarrow 0$.

Com efeito, supondo válida esta afirmação, a expressão (3.6.2) assume a forma:

$$
\frac{a r_{0}(k) c_{1}+c_{1}-a c_{2} c_{3}}{a r_{0}(k)+1}
$$

a qual em módulo é menor ou igual que:

$$
\frac{a \rho_{0}\left|c_{1}\right|+\left|c_{1}\right|+a\left|c_{2} c_{3}\right|}{1-a \rho_{0}}
$$

que é limitada, ainda no limite $r_{0}(k) \rightarrow \infty$. Aqui as $c_{i}$ são certas magnitudes limitadas.

Para provar a afirmação, observe que o caso $i=1$ está contemplado em 3.4.3. Para o caso $i=2$ a prova é como a de 3.4.2(a) (que teve 3.4.3 como corolário). Usando 3.4.1(b) a integral em questão se reduz a:

$$
2 \pi \int Z^{2}(\mathbf{q}) \operatorname{senh} \omega(\mathbf{q}) \cosh \omega(\mathbf{q}) \frac{f_{2}(\mathbf{q})}{\cosh ^{2} \omega(\mathbf{q})-\cos ^{2} k_{0}} d \mathbf{q}
$$

O problema consiste em controlar a divergência do denominador no integrando, e a idéia para isto é usar as propriedades da $f_{2}$. Começamos quebrando a integral acima em duas regiões, $|\mathbf{q}| \geqslant \epsilon$ e $|\mathbf{q}|<\epsilon$, para um dado $\epsilon>0$ convenientemente pequeno. $\mathrm{O}$ integrando não tem singularidades na primeira região para $y_{0}$ numa vizinhança de $m$ e a integral correspondente não apresenta problemas. Na segunda região, supondo que $\omega^{2}(\mathbf{q}) \approx m^{2}+c|\mathbf{q}|^{2}$ perto da origem, tem-se:

$$
\frac{1}{\cosh ^{2} \omega(\mathbf{q})-\cos ^{2} k_{0}} \approx \frac{1}{\omega^{2}(\mathbf{q})+k_{0}^{2}} \approx \frac{1}{m^{2}+c|\mathrm{q}|^{2}+k_{0}^{2}} .
$$

Seja $f_{3}$ a função definida pela seguinte identidade:

$$
f(0, \mathbf{q})=f(0)+\left.\mathbf{q} \cdot \operatorname{grad} f\right|_{\mathbf{q}=0}+f_{3}(\mathbf{q}) \text {. }
$$


Usando a simetria $p \longleftrightarrow-p$, o integrando se reduz a:

$$
\frac{f_{3}(\mathbf{q})}{m^{2}+c|\mathbf{q}|^{2}+k_{0}^{2}}
$$

o qual não diverge para $k_{0}$ numa vizinhança de $i m$, pois $f_{3}(\mathbf{q})$ é da ordem de $|\mathbf{q}|^{2}$.

\subsection{O Caso de Dimensão $1+1$}

Nas três seções anteriores foram desenvolvidos resultados gerais e de validade irrestrita, dentro das suas hipóteses. Para poder engastar-los na trama da completeza assintótica devemos restringir-nos, porém, ao caso $d=1$, ou seja, duas dimensões. Isto introduz vantagens pura e exclusivamente técnicas, sem constituir-se, até onde chega nosso conhecimento, numa restrição imposta pelo caráter intrinsecamente físico inerente à situação. As condições impostas recaem direta o indiretamente sobre a relação de dispersão. A restrição para duas dimensões acarreta simples porém não triviais mudanças concernentes a:

- o domínio de analiticidade,

- a região absolutamente contínua do espectro.

No primeiro caso, a restrição a duas dimensões, mais a introdução de uma hipótese conveniente sobre a relação de dispersão, faz com que a integral em $d$ p da expressão em 3.4.1(b) vire uma integral em um intervalo real, suscetível de ser deformado a um contorno no plano complexo. A natureza da hipótese extra faz com que a região $\left\{k_{0}=i y_{0}: m \leqslant\left|y_{0}\right|<m+\delta_{0}\right\}$ possa ser incluída no domínio de analiticidade no contexto de 3.4.2(a). No segundo caso, a restrição a duas dimensões possibilita que a relação de dispersão possa ser "invertida", ao menos formalmente, permitindo obter uma derivada de Radon-Nikodym da medida espectral. Unindo estes fatos com 3.6.1, pode-se obter completeza assintótica em uma região de energias que vão até $2\left(m+\delta_{0}\right)$. Sem a hipótese que possibilita a mudança do domínio de analiticidade, obteria-se também completeza assintótica mas neste caso apenas até "um pouquinho" além de $2 m$.

Na presente seção vamos supor $d=1$ e conseqüentemente denotaremos $\mathrm{p}=p_{1}$. Introduzimos a seguinte suposição:

S1 Existe $\alpha>0$ tal que a relação de dispersão $\omega\left(p_{1}\right)$ admite uma extensão analítica $\omega\left(p_{1}+i q_{1}\right)$ à faixa $\left|q_{1}\right|<\alpha$ com a seguinte propriedade: Existe 
um caminho $t: I \longmapsto \mathbb{C}$ contido nessa faixa, com $t(I) \cap \mathbb{R} \subseteq\{0\}$, tal que:

$$
i \omega(t(I)) \cap\left\{k_{0}=i y_{0}: 0<\left|y_{0}\right|<m+\delta_{0}\right\} \subseteq\{i m\} .
$$

3.7.1 Lema: Sob S1, os lemas 3.4.1(c), 3.4.2(a) e 3.4.5 valem numa vizinhança de $\left\{k_{0}=i y_{0}:\left|y_{0}\right|<m+\delta_{0}\right\}$, exceto possivelmente em uma vizinhança de $k_{0}=i m$ que pode ser escolhida arbitrariamente pequena.

Demonstração: É análoga à de 3.4.2(a). Usando 3.4.1(b), deve ser verificada a analiticidade de:

$$
\pi \int_{-\pi}^{\pi} \frac{Z\left(p_{1}+k_{1}\right) Z\left(p_{1}-k_{1}\right) \operatorname{senh}\left[\omega\left(p_{1}+k_{1}\right)+\omega\left(p_{1}-k_{1}\right)\right]}{a^{2}\left(p_{1}\right)-\cos ^{2} k_{0}} f\left(0, p_{1}\right) d p_{1} .
$$

Aqui, novamente, a idéia consiste em deslocar o caminho da integração em $d p_{1}$ para o caminho $t(s)$ no plano complexo. A região de analiticidade deverá excluir os $k_{0}$ que jazem sobre este caminho. A escolha de S1 libera os $\left\{k_{0}=i y_{0}: m \leqslant\left|y_{0}\right|<m+\delta_{0}\right\}$, exceto uma vizinhança de $k_{0}=i m$. Com efeito, usando a identidade trigonométrica:

$$
\cos ^{2} a-\cos ^{2} b=-\operatorname{sen}(a+b) \operatorname{sen}(a-b)
$$

o denominador do integrando acima é dado por:

$$
\begin{aligned}
\cosh ^{2} \omega(t(s))-\cos ^{2} k_{0}=\cos ^{2} i \omega(t(s)) & -\cos ^{2} k_{0} \\
& =\operatorname{sen}\left[k_{0}+i \omega(t(s))\right] \operatorname{sen}\left[k_{0}-i \omega(t(s))\right],
\end{aligned}
$$

e, pela propriedade $\mathrm{S} 1$, esta última expressão é não nula para tais $k_{0}$, exceto possivelmente para $k_{0}=i m$.

A suposição S1 é melhor comprendida quando a relação de dispersão é conhecida explicitamente. Veja-se [101] p. 9 para um exemplo no caso $\omega\left(p_{1}\right)=\sqrt{4 m^{2}+p_{1}^{2}}$. De fato, a condição $\mathrm{S} 1$ foi formulada tendo este exemplo como paradigmal.

Por 3.7.1 temos que, salvo uma vizinhança de $k_{0}=i m$ (que pode ser escolhida arbitrariamente pequena), a região $\left\{k_{0}=i y_{0}: m \leqslant y_{0}<m+\delta_{0}\right\}$ não contém pontos de acumulação de polos, podendo, sim, conter polos. Temos assim um resultado analogo de 3.5.1(a):

3.7.2 Lema: Seja $f \in A_{\delta}$. Denotemos $\cos k_{0}-1=x+i y$.

(a) Seja $(\alpha, \beta) \subseteq\left(2 m, 2\left(m+\delta_{0}\right)\right)$ e suponha-se que $R\left(k_{0}, 0\right)$ não tem polos em $\left\{k_{0}=i y_{0}: y_{0} \in(\alpha, \beta)\right\}$. Então a distribuição dada por:

$$
h \longmapsto \lim _{y \rightarrow 0^{+}} \int \operatorname{Im}\left\langle f, R\left(k_{0}, 0\right) f\right\rangle h(x) d x
$$


está bem definida em $\mathcal{D}(\cosh \alpha-1, \cosh \beta-1)$ estando dada por:

$$
h \longmapsto 4 \pi^{3} \int_{-\pi}^{\pi} Z^{2}\left(p_{1}\right) \operatorname{senh} \omega\left(p_{1}\right) W f\left(0, p_{1}\right) \overline{W f}\left(0, p_{1}\right) h\left(\cosh \omega\left(p_{1}\right)-1\right) d p_{1},
$$

onde:

$$
W f:=\lim _{\substack{y \rightarrow 0^{+} \\ x \rightarrow \cosh \omega\left(p_{1}\right)-1}}\left[I+K\left(k_{0}, 0\right) R_{0}\left(k_{0}, 0\right)\right]^{-1} f .
$$

(b) Em particular, sob as hipóteses da parte (a), tem-se:

$$
d\left\langle\Theta(\check{f}), E_{\left(\lambda_{0}, 0\right)} \Theta(\check{g})\right\rangle_{\mathcal{H}}=\left|L f\left(\lambda_{0}\right)\right|^{2} d \lambda_{0}
$$

quando $\lambda_{0} \in(2 \alpha, 2 \beta)$, e onde:

$$
L f\left(\lambda_{0}\right):=\left[\pi\left(\omega^{-1}\right)^{\prime}\left(\lambda_{0} / 2\right)\right]^{1 / 2}(Z W f)\left(\omega^{-1}\left(\lambda_{0} / 2\right)\right) .
$$

Demonstração: A parte (a) é como em 3.5.1(b). Para a parte (b), observe que se $d=1$, a relação de dispersão pode inverter-se, ao menos localmente. Ou seja, na segunda integral da parte (a) podemos efetuar a mudança de variáveis dada por:

$$
a:=\cosh \omega\left(p_{1}\right)-1
$$

em cujo caso $d a=\operatorname{senh} \omega\left(p_{1}\right) \omega^{\prime}\left(p_{1}\right) d p_{1}$, ou:

$$
\operatorname{senh} \omega\left(p_{1}\right) d p_{1}=\frac{d a}{\omega^{\prime}\left[\omega^{-1}(\operatorname{arcosh}(a+1))\right]}=\left(\omega^{-1}\right)^{\prime}(\operatorname{arcosh}(a+1)) d a .
$$

Com isto, a integral fica:

$$
4 \pi^{3} \int_{\cosh \alpha-1}^{\cosh \beta-1}\left(Z^{2} W f \overline{W f}\right)\left[\omega^{-1}(\operatorname{arcosh}(a+1))\right]\left(\omega^{-1}\right)^{\prime}(\operatorname{arcosh}(a+1)) h(a) d a .
$$

Comparando isto com (3.3.3), temos:

$$
\begin{aligned}
& d\left\langle\Theta(\check{f}), E_{(2 \operatorname{arcosh}(a+1), 0)} \Theta(\check{f})\right\rangle_{\mathcal{H}} \\
& \quad=2 \pi \frac{\left(Z^{2} W f \overline{W f}\right)\left[\omega^{-1}(\operatorname{arcosh}(a+1))\right]\left(\omega^{-1}\right)^{\prime}(\operatorname{arcosh}(a+1))}{\sqrt{(a+1)^{2}-1}} d a .
\end{aligned}
$$

A mudança de variáveis $\lambda_{0}=2 \operatorname{arcosh}(a+1)$, permite transcrever esta última identidade na fórmula do enunciado. 
3.7.3 Lema: Denotemos por $\sigma$ o espectro do operador de energia-momento restrito ao subespaço:

$$
\overline{\operatorname{span}}\left\{\Theta(\check{f}): f \in A_{\delta}^{1}\right\} \cap \mathcal{H}^{2\left(m+\delta_{0}\right)} .
$$

Então: $(0,2 m) \cap \sigma \subseteq-2 i\left[S \cap\left\{k_{0}=i y_{0}: y_{0} \in(0, m)\right\}\right]$.

Demonstração: É imediato de 3.5.2.

3.7.4 Lema: Se a constante a de H3 é suficientemente pequena, então o operador $R\left(k_{0}, 0\right)$ não tem polos em $\left\{k_{0}=i y_{0}: 0<y_{0}<m+\delta_{0}\right\}$.

Demonstração: É análoga à de 3.6.1, mas desta vez considerando 3.7.1.

3.7.5 Lema: Denotemos por $\sigma$ o espectro do operador de energia-momento restrito ao subespaço:

$$
\overline{\operatorname{span}}\left\{\Theta(\check{f}): f \in A_{\delta}^{1}\right\} \cap \mathcal{H}^{2\left(m+\delta_{0}\right)} .
$$

Então: $\left(2 m, 2\left(m+\delta_{0}\right)\right) \cap \sigma$ é absolutamente contínuo e de multiplicidade 1.

Demonstração: Observe que de 3.7 .4 e $3.7 .2(\mathrm{~b})$ segue que a aplicação $\Theta(\check{f}) \longmapsto$ $L f$ de $\left\{\Theta(\check{f}): f \in A_{\delta}^{1}\right\} \cap \mathcal{H}^{2\left(m+\delta_{0}\right)}$ em $L^{2}\left(\left(2 m, 2\left(m+\delta_{0}\right)\right), d \lambda_{0}\right)$ é unitária, podendo ser extendida a uma aplicação de $\overline{\operatorname{span}}\left\{\Theta(\check{f}): f \in A_{\delta}^{1}\right\} \cap \mathcal{H}^{2\left(m+\delta_{0}\right)}$ em $L^{2}\left(\left(2 m, 2\left(m+\delta_{0}\right)\right), d \lambda_{0}\right)$, e que neste último espaço o operador de energia-momento age como o operador de multiplicação $f\left(\lambda_{0}\right) \longmapsto \lambda_{0} f\left(\lambda_{0}\right)$.

Lembrando a notação introduzida antes do enunciado de 3.5 .2 , p. 60, seja $\mathcal{H}_{d}$ o subespaço fechado de $\mathcal{H}$ generado pelos autovetores do operador de energiamomento, e denotemos:

$$
\begin{aligned}
\mathcal{H}_{d}^{a} & :=\mathcal{H}_{d} \cap \mathcal{H}^{a} \\
\mathcal{H}_{\text {in }}^{a} & :=\mathcal{H}_{\text {out }} \cap \mathcal{H}^{a} .
\end{aligned}
$$

Por conveniência, introduzimos também a seguinte hipótese:

H4 $\overline{\operatorname{span}}\left\{\Theta(\check{f}): f \in A_{\delta}^{1}\right\} \supseteq \mathcal{H}^{2\left(m+\delta_{0}\right)}$.

Incidentalmente, observe-se que:

$$
\begin{aligned}
& \overline{\operatorname{span}}\left\{\Theta(\check{f}): f \in A_{\delta}^{1} \wedge \operatorname{supp} \check{f} \text { é compacto }\right\} \\
& \quad=\overline{\operatorname{span}}\left\{\Theta(\check{f}): f \in A_{\delta}^{1}\right\}=\overline{\operatorname{span}}\left\{\Theta(\check{f}): f \in A_{\delta}\right\} .
\end{aligned}
$$


Resumindo o feito nesta seção, temos então o seguinte resultado final:

3.7.6 Teorema: Sendo satisfeitas as hipóteses H1, H2, e H4, e se $d=1$, tem-se:

(a) $\mathcal{H}^{2\left(m+\delta_{0}\right)}=\mathcal{H}_{d}^{2\left(m+\delta_{0}\right)} \oplus \mathcal{H}_{i n}^{2\left(m+\delta_{0}\right)}$ out

(b) Se H3 também é satisfeita, então $\mathcal{H}^{2\left(m+\delta_{0}\right)}=\mathcal{H}_{\substack{\text { in } \\ \text { out }}}^{2\left(m+\delta_{0}\right)}$.

Demonstração: A parte (a) segue de 3.7.5 e do teorema espectral. A parte (b) segue de 3.7.3 e 3.7.4.

\subsection{O Caso de Momento Não-Nulo}

Apresentamos nesta seção a generalização de alguns resultados das seções anteriores para o caso de momento não nulo. Isto é feito numa seção separada, dado que em certos casos devemos acrescentar hipóteses adicionais, apenas pequenos detalhes técnicos, quase todas envolvendo a relação de dispersão. Este problema não existe no contínuo, ${ }^{25}$ onde, por causa da invariância de Lorentz, da qual a teoria na rede carece, basta considerar apenas o caso $\mathrm{k}=0$. Um outro motivo para a abordagem em separado origina-se no fato de que em determinadas circunstâncias as provas aqui apresentadas não estão completas.

Observe-se que 3.4.1 é um resultado válido para o caso de momento arbitrário. A generalização de 3.4.2(a) apresenta algumas dificuldades. Como antes, a região $\left|y_{0}\right|<m$ não apresenta problemas. Na região $m \leqslant\left|y_{0}\right|<m+\delta_{0}$ as considerações sobre as funções $F_{ \pm}$são as mesmas, mas agora o primeiro somando em 3.4.4 é dado por: ${ }^{26}$

$$
\int_{T^{d+1}} \frac{Z(\mathrm{p}+\mathrm{k}) \operatorname{senh}[\omega(\mathrm{p}+\mathrm{k})] Z(\mathrm{p}-\mathrm{k}) \operatorname{senh}[\omega(\mathrm{p}-\mathrm{k})]}{[\cosh \omega(\mathrm{p}-\mathrm{k})-\cosh \omega(\mathrm{p}+\mathrm{k})]-2 \operatorname{sen} k_{0} \operatorname{sen} p_{0}} f_{1}(p) F_{+}(p) d p .
$$

Observando que $\cosh a=\cos i a=2 \cos ^{2}(i a / 2)-1$ podemos usar a identidade trigonométrica (3.7.1) que aparece na prova de 3.7.1 para obter a seguinte:

$$
\cosh a-\cosh b=-2 \operatorname{sen}(i(a+b) / 2) \operatorname{sen}(i(a-b) / 2),
$$

\footnotetext{
${ }^{25}$ Cf. [101] p. 8.

${ }^{26} \mathrm{O}$ caso do segundo somando é análogo.
} 
a qual permite reescrever o somando entre parênteses do denominador no integrando acima, resultando a integral em questão:

$$
\frac{1}{2} \int_{T^{d+1}} \frac{Z(\mathrm{p}+\mathrm{k}) \operatorname{senh}[\omega(\mathrm{p}+\mathrm{k})] Z(\mathrm{p}-\mathrm{k}) \operatorname{senh}[\omega(\mathrm{p}-\mathrm{k})]}{\operatorname{sen}(i a) \operatorname{sen}(i b)-\operatorname{sen} k_{0} \operatorname{sen} p_{0}} f_{1}(p) F_{+}(p) d p,
$$

onde denotamos:

$$
\begin{aligned}
a & :=\frac{\omega(\mathrm{p}+\mathrm{k})+\omega(\mathrm{p}-\mathrm{k})}{2} \\
b & :=\frac{\omega(\mathrm{p}+\mathrm{k})-\omega(\mathrm{p}-\mathrm{k})}{2} .
\end{aligned}
$$

O denominador no integrando acima introduz uma nova divergência que já não pode ser controlada de maneira simples com a analiticidade da $f_{1}$ como no caso do momento nulo. Para isto, quebramos a integral em $d p_{0}$ em duas regiões, $\left|p_{0}\right|<\epsilon \mathrm{e}$ $\left|p_{0}\right| \geqslant \epsilon$. Observe-se que $a \geqslant m$ e $|b|<\delta_{0} / 2$ pelo que o denominador no integrando é nulo quando:

$$
\left\{\begin{array}{l}
k_{0}=i a \\
p_{0}=i b
\end{array}\right.
$$

Assim, na segunda região podemos deslocar o caminho de integração como no caso de momento nulo. Por outro lado, observe-se também que $\epsilon$ pode ser escolhido suficientemente pequeno como para que a identidade $p_{0}+x_{0}=0$ não possa ser satisfeita na primeira região (lembre-se que $k_{0}=x_{0}+i y_{0}$ é arbitrário mas fixo). Assim, na primeira região as dificuldades que conspiram contra a analiticidade provêm apenas do denominador do primeiro fator no integrando. Se $p_{0}$ é real e $\left|x_{0}\right|<\pi / 2$ (o que não constitui restrição nenhuma (Cf. 3.4.5(b))) o denominador apenas pode ser nulo se $p_{0}=k \pi, k=0,1$, em cujo caso o denominador é nulo se e somente se $b=k \pi$. Neste caso, observe-se que:

$$
|\operatorname{sen}(i b)| \approx \frac{1}{2}|\omega(\mathrm{p}+\mathrm{k})-\omega(\mathrm{p}-\mathrm{k})| \approx|\nabla \omega(\mathrm{k})||\mathrm{p}| .
$$

Portanto, supondo que, por exemplo, $\nabla \omega(\mathrm{k})=0 \Longrightarrow \mathrm{k}=0$, podemos controlar a divergência com uma adequada condição sobre a $f_{1}$.

A generalização de 3.4 .2 (b) consiste igualmente em provar que a contribuição à distribuição:

$$
\begin{array}{r}
h \longmapsto(2 \pi)^{\frac{d+1}{2}} \pi^{2} \int_{T^{d}} Z(\mathrm{p}+\mathrm{k}) Z(\mathrm{p}-\mathrm{k}) \operatorname{senh}[\omega(\mathrm{p}+\mathrm{k})+\omega(\mathrm{p}-\mathrm{k})] \\
\times f(0, \mathrm{p}) \frac{h(a(\mathrm{p})-1)}{a(\mathrm{p})} d \mathrm{p}
\end{array}
$$


é nula, onde, como antes,

$$
a(\mathrm{p}):=\sqrt{\frac{\cosh [\omega(\mathrm{p}+\mathrm{k})+\omega(\mathrm{p}-\mathrm{k})]+1}{2}} .
$$

Denotando:

$$
\begin{aligned}
& a_{+}:=\cosh \omega(\mathrm{p}+\mathrm{k}) \\
& a_{-}:=\cosh \omega(\mathrm{p}-\mathrm{k}),
\end{aligned}
$$

temos:

$$
\begin{gathered}
\operatorname{Im}\left(\frac{1}{a_{+}-\cos \left(p_{0}+k_{0}\right)} \frac{1}{a_{-}-\cos \left(p_{0}-k_{0}\right)}\right) \\
\quad=\frac{-2 y\left[(x+1)-a \cos p_{0}\right]+2 b v(x, y) \operatorname{sen} p_{0}}{A^{2}+B^{2}} .
\end{gathered}
$$

Aqui denotamos:

$$
\begin{aligned}
A & =\left[(x+1)-a \cos p_{0}\right]^{2}+\left(a^{2}-1\right) \operatorname{sen}^{2} p_{0}-y^{2}-b^{2}-2 b u(x, y) \operatorname{sen} p_{0} \\
B & =2 y\left[(x+1)-a \cos p_{0}\right]-2 b v(x, y) \operatorname{sen} p_{0} \\
a & =\frac{a_{+}+a_{-}}{2} \\
b & =\frac{a_{+}-a_{-}}{2}
\end{aligned}
$$

onde $u(x, y)$ e $v(x, y)$ denotam as partes real e imaginária de

$$
\sqrt{1-[(x+1)+i y]^{2}}=\operatorname{sen} k_{0}
$$

respectivamente. Observe que $u \rightarrow 0$ e $v \rightarrow \sqrt{x(x+2)}$ quando $y \rightarrow 0^{+}$. Como antes, temos:

$$
\begin{aligned}
& \lim _{y \rightarrow 0^{+}} \int_{0}^{\infty} \operatorname{Im}\left(\int_{T^{d+1}} R_{00}\left(\left(k_{0}, 0\right), p\right) f_{1}(p) d p\right) h(x) d x \\
& =\int_{T^{d}} d \mathrm{p} Z(\mathrm{p}+\mathrm{k}) \operatorname{senh}[\omega(\mathrm{p}+\mathrm{k})] Z(\mathrm{p}-\mathrm{k}) \operatorname{senh}[\omega(\mathrm{p}-\mathrm{k})] \\
& \times\left(\lim _{y \rightarrow 0^{+}} \int_{-\pi}^{\pi} \int_{0}^{\infty} \frac{-2 y\left[(x+1)-a \cos p_{0}\right] h(x) f_{1}\left(p_{0}, \mathrm{p}\right) d x d p_{0}}{A^{2}+B^{2}}\right. \\
& \left.+\lim _{y \rightarrow 0^{+}} \int_{-\pi}^{\pi} \int_{0}^{\infty} \frac{2 b v(x, y) \operatorname{sen} p_{0} h(x) f_{1}\left(p_{0}, \mathrm{p}\right) d x d p_{0}}{A^{2}+B^{2}}\right) .
\end{aligned}
$$


Afirmamos que o segundo dos somandos entre parênteses acima é nulo. Com efeito, "no limite $y \rightarrow 0^{+}$" o integrando é uma função ímpar de $p_{0}$, sendo nula portanto a sua integral entre $-\pi$ e $\pi$. Afirmamos igualmente que o primeiro somando é proporcional a $f_{1}(0, \mathbf{p})$ que também é nulo pela definição de $f_{1}$. Com efeito, um argumento de plausibilidade é o seguinte: quando $y \rightarrow 0^{+}$o denominador converge para uma função de $x$ e $p_{0}$ que é nula se e somente se:

$$
\left\{\begin{array}{l}
{\left[(x+1)-a \cos p_{0}\right]^{2}+\left(a^{2}-1\right) \operatorname{sen}^{2} p_{0}-b^{2}=0} \\
-2 b \sqrt{x(x+2)} \operatorname{sen} p_{0}=0
\end{array}\right.
$$

Se $x>0$ e $b \neq 0$ (se $b=0$ a prova se reduz ao caso de momento nulo), a segunda identidade acima é satisfeita se e somente se $p_{0}=0$, em cujo caso a primeira se reduz a:

$$
[(x+1)-a]^{2}-b^{2}=0,
$$

de onde:

$$
x+1=\frac{ \pm\left|a_{+}-a_{-}\right|}{2}+\frac{a_{+}+a_{-}}{2}=a_{ \pm} .
$$

Assim, é de se esperar que o integrando convirja para:

$$
\text { const }\left[\delta\left(x+1-a_{+}\right)+\delta\left(x+1-a_{-}\right)\right] \delta\left(p_{0}\right) .
$$

A generalização de 3.4.5 para o caso de $\mathrm{k} \neq 0$ é imediata, e igualmente a de 3.5.1 e 3.5.2 (as provas são análogas às do caso de momento nulo). A de 3.6.1 também não apresenta problemas: usando 3.4.1(b) a integral em questão se reduz a:

$$
2 \pi \int \frac{Z(\mathbf{q}+\mathbf{k}) Z(\mathbf{q}-\mathbf{k}) \operatorname{senh}[\omega(\mathbf{q}+\mathbf{k})+\omega(\mathbf{q}-\mathbf{k})]}{\cosh [\omega(\mathbf{q}+\mathbf{k})+\omega(\mathbf{q}-\mathbf{k})]-\cos 2 k_{0}} f_{2}(\mathbf{q}) d \mathbf{q} .
$$

Supondo igualmente que $\omega(\mathbf{q}) \approx m+c|\mathrm{q}|^{2}$ perto da origem, e observando que:

$$
|\mathbf{q}+\mathbf{k}|^{2}+|\mathbf{q}-\mathrm{k}|^{2}=2\left(|\mathrm{q}|^{2}+|\mathrm{k}|^{2}\right),
$$

tem-se:

$$
\frac{\omega(\mathbf{q}+\mathbf{k})+\omega(\mathbf{q}-\mathbf{k})}{2} \approx m+c\left(|\mathbf{q}|^{2}+|\mathbf{k}|^{2}\right) .
$$

Assim, analogamente:

$$
\begin{aligned}
& \frac{1}{\cosh [\omega(\mathbf{q}+\mathrm{k})+\omega(\mathbf{q}-\mathrm{k})]-\cos 2 k_{0}} d \mathbf{q}=\frac{1 / 2}{\cosh ^{2}\left[\frac{\omega(\mathrm{q}+\mathrm{k})+\omega(\mathrm{q}-\mathrm{k})}{2}\right]-\cos ^{2} k_{0}} \\
& \approx \frac{1}{\left[\frac{\omega(\mathrm{q}+\mathrm{k})+\omega(\mathrm{q}-\mathrm{k})}{2}\right]^{2}+k_{0}^{2}} \approx \frac{1}{\left[m+c\left(|\mathbf{q}|^{2}+|\mathrm{k}|^{2}\right)\right]^{2}+k_{0}^{2}}
\end{aligned}
$$


Esta última expressão não diverge para $k_{0}$ numa vizinhança de $i m$, a menos que $\mathrm{k}=0$, mas para este caso vale a prova de 3.6.1 apresentada na seção 3.6.

O lema 3.7.1 foi provado no caso de momento arbitrário, e a generalização de 3.7.2 também não apresenta problemas. Neste caso, por (3.8.1), a integral de 3.7.2(a) assume a forma:

$$
\begin{aligned}
& 2 \pi^{3} \int_{-\pi}^{\pi} Z\left(p_{1}+k_{1}\right) Z\left(p_{1}-k_{1}\right) \operatorname{senh}\left[\omega\left(p_{1}+k_{1}\right)+\omega\left(p_{1}-k_{1}\right)\right] \\
& \times W f\left(0, p_{1}\right) \overline{W f}\left(0, p_{1}\right) \frac{h\left(a\left(p_{1}\right)-1\right)}{a\left(p_{1}\right)} d p_{1}
\end{aligned}
$$

onde:

$$
\begin{aligned}
a\left(p_{1}\right) & =\sqrt{\frac{\cosh \left[\omega\left(p_{1}+k_{1}\right)+\omega\left(p_{1}-k_{1}\right)\right]+1}{2}} \\
W f & =\lim _{\substack{y \rightarrow 0^{+} \\
x \rightarrow a\left(p_{1}\right)-1}}\left[I+K\left(k_{0}, k_{1}\right) R_{0}\left(k_{0}, k_{1}\right)\right]^{-1} f .
\end{aligned}
$$

Para manter a analogia com o caso de momento nulo definimos:

$$
a:=a\left(p_{1}\right)-1,
$$

e vamos supor que a função $F$ definida por:

$$
F\left(p_{1}\right):=\omega\left(p_{1}+k_{1}\right)+\omega\left(p_{1}-k_{1}\right)=\operatorname{arcosh}\left[2(a+1)^{2}-1\right]=: \mu(a)
$$

é invertível e a derivada de $F^{-1}$ é positiva. Neste caso temos:

$$
4(a+1) d a=\operatorname{senh}\left(F\left(p_{1}\right)\right) F^{\prime}\left(p_{1}\right) d p_{1},
$$

de onde:

$$
\operatorname{senh}\left(F\left(p_{1}\right)\right) d p_{1}=4(a+1)\left(F^{-1}\right)^{\prime}(\mu(a)) d a,
$$

e assim, com esta mudança de variáveis, (3.8.2) resulta em:

$$
\begin{aligned}
8 \pi^{3} \int_{-\pi}^{\pi} Z\left(F^{-1}(\right. & \left.\mu(a))+k_{1}\right) Z\left(F^{-1}(\mu(a))-k_{1}\right) \\
& \times W f\left[0, F^{-1}(\mu(a))\right] \overline{W f}\left[0, F^{-1}(\mu(a))\right]\left(F^{-1}\right)^{\prime}(\mu(a)) h(a) d a .
\end{aligned}
$$

Daqui, a comparação com (3.3.3) e um cálculo direto conduzem a:

$$
L f\left(\lambda_{0}\right)=\left[2 \pi Z\left(F^{-1}\left(\lambda_{0}\right)+k_{1}\right) Z\left(F^{-1}\left(\lambda_{0}\right)-k_{1}\right)\left(F^{-1}\right)^{\prime}\left(\lambda_{0}\right)\right]^{1 / 2} W f\left(0, F^{-1}\left(\lambda_{0}\right)\right) .
$$

Os demais resultados da seção 3.7 valem no caso geral. 


\section{Parte II}

\section{O Modelo de Ising na Região de Altas Temperaturas}




\section{Capítulo 4}

\section{Expansão em Polímeros}

\subsection{Introdução}

Sendo talvez o sistema mais simples em exibir uma transição de fase não trivial, o modelo de Ising ocupa um lugar destacado, quase único, na física teórica, amplamente sustentado por uma vastíssima literatura. E. Ising, no seu trabalho seminal ${ }^{1}$ de 1925, baseado basicamente na sua tese de doutorado, concede a Wilhelm Lenz, seu orientador, o crédito pela invenção do modelo, mas seu nome, curiosamente, nunca foi associado com o modelo desde então.

Proposto originalmente como modelo para o ferromagnetismo, consiste basicamente no seguinte: em cada sítio de uma rede, v.g., $\mathbb{Z}^{d+1}$, tem-se spins com duas possíveis orientações, 1 (para cima) ou -1 (para baixo), ou seja, consideram-se configurações em $\{1,-1\}^{\Lambda}$. Numa dada configuração $\sigma$ a energia de interação é dada por:

$$
H_{\Lambda}(\sigma)=-j \sum_{\substack{i, j \in \Lambda \\|i-j|=1}} \sigma(i) \sigma(j)-h \sum_{i \in \Lambda} \sigma(i) .
$$

Aqui, $j$ é a "constante de acoplamento" e $h$ representa a grandeza de um campo magnético externo. O primeiro somando no membro direito acima corresponde à energia de interação entre os spins, sendo esta apenas entre os "vizinhos próximos", e o segundo à interação entre os spins individuais e o campo magnético. A energia de interação entre dois vizinhos próximos é:

$$
-j \sigma(i) \sigma(j)= \begin{cases}-j, & \text { se } \sigma(i)=\sigma(j), \\ j, & \text { se } \sigma(i) \neq \sigma(j) .\end{cases}
$$

${ }^{1}[55]$. Para as referências originais dos trabalhos dos outros autores citados nesta seção veja-se [105], cujo capítulo 5 seguimos nesta breve introdução. 
Assim, se $j>0$, a energia tende a ser menor nas configurações de spins paralelos. A menor de todas, a energia do "estado fundamental", é dada neste caso $(j>0)$ por:

$$
-j|\mathcal{V}(\Lambda)|-h|\Lambda|
$$

sendo atingida quando todos os spins estão para cima (resp. abaixo) se $h>0$ (resp. $h<0)$. Aqui $\mathcal{V}(\Lambda)$ é o conjunto de todos os vizinhos próximos em $\Lambda$ e a expressão é válida para condições de contorno periódicas. Como as configurações de spins paralelos propiciam uma menor energia, é dito que o caso $j>0$ corresponde ao ferromagnetismo. Analogamente, o caso $j<0$ corresponde ao antiferromagnetismo, pois a menor energia é favorecida neste caso pelas configurações de spins antiparalelos.

O problema da mecânica estatística consiste basicamente em calcular a "função partição" do sistema:

$$
Z_{\Lambda}=\sum_{\sigma \in\{1,-1\}^{\Lambda}} \mathrm{e}^{-\beta H_{\Lambda}(\sigma)}
$$

Ising resolveu exatamente o modelo em uma dimensão, verificando a ausência de ferromagnetismo neste caso. Além disso, enumerou algumas das causas que fariam com que esta situação se repetisse em duas e 3 dimensões (o qual, sabe-se agora, não é o caso), e conseqüentemente o modelo caiu no esquecimento. Foi posteriormente retomado nos anos 30 por Bragg e Williams, Bethe, Peierls e outros, mas principalmente como modelo para ligas binárias (1 significando um átomo do tipo $\mathrm{A},-1 \mathrm{um}$ átomo do tipo B) mais do que um modelo para um material ferromagnético. Peierls forneceu uma prova, infelizmente incorreta, da existência de ferromagnetismo para o modelo em 2 dimensões. O erro foi percebido só mais tarde, por Fisher e Sherman, e a prova foi completada rigorosamente por Griffiths em 1964. A maior inovação veio de Kramers e Wannier, que em 1941 formularam o problema matricialmente e localizaram corretamente, por considerações de simetria, o ponto crítico de maneira exata em duas dimensões. Porém, não resolveram o problema completamente. Foi Onsager quem em 1944 obteve a solução completa (i.e., a função partição) para o modelo em duas dimensões na ausência de campo magnético externo. ${ }^{2}$ Esta solução foi de fato a primeira prova não trivial da existência de uma transição de fase a partir da função partição. Após o trabalho de Onsager os artigos começaram a suceder-se continuamente. Revisões e desenvolvimentos posteriores estão bem documentados e podem consultar-se nos trabalhos de Brush ${ }^{3}$ e McCoy e Wu. ${ }^{4}$

\footnotetext{
${ }^{2}[71]$

${ }^{3}[13]$.

${ }^{4}[64]$.
} 
O problema em uma dimensão com $h=0$ é bem simples. O caso $h \neq 0$ (com condições periódicas de contorno) pode ser resolvido pelo método da matriz de transferência, que tem a vantagem de poder ser generalizado para duas ou mais dimensões. O problema em duas dimensões no caso $h=0$, como já foi dito, foi resolvido por Onsager num dos artigos mais celebrados de seu tempo. $\mathrm{O}$ caso $h \neq 0$ e o problema em 3 dimensões, ainda para $h=0$, permanecem em aberto até hoje. O problema em duas dimensões foi reformulado e resolvido por vários autores numa variedade de maneiras, na esperança de que uma nova abordagem ilumine o caminho até uma solução do problema em 3 dimensões. Entanto este anelo espera ainda pela sua materialização, muitos resultados interessantes e novas inter-relações com outras áreas da matemática apareceram. Notável entre elas é a relação entre o modelo de Ising e problemas combinatórios. Alguns novos resultados combinatórios surgiram por esta via, embora pouco tenha sido acrescentado, infelizmente, à compreensão do problema de Ising. Finalmente, é oportuno acrescentar que o modelo de Ising tem certa relevância na Teoria Quântica de Campos, pois um dos seus principais modelos, a teoria $P(\phi)_{2}$, pode ser aproximado por aquele. ${ }^{5}$

No presente trabalho focalizamos nossa atenção no modelo de Ising, não tanto pela relevância que possa ter, mas pela relativa simplicidade do mesmo, e para exemplificar técnicas que admitem aplicação em uma grande variedade de outras circunstâncias.

\subsection{O Modelo de Ising}

Dada a natureza indutiva ${ }^{6}$ da álgebra de observáveis $C\left(S^{\mathbb{Z}^{d+1}}\right)$ é razoável supor que um estado $\mu \in S_{C\left(S^{d}{ }^{d+1}\right)}$ possa ser definido e/ou ficar determinado a partir de uma coleção de "estados locais" $\mu_{\Lambda} \in S_{C\left(S^{\Lambda}\right)}$. Esta é uma questão delicada cujos detalhes não vamos aprofundar. ${ }^{7}$ Observemos apenas o seguinte: cada estado $\mu_{\Lambda} \in S_{C\left(S^{\Lambda}\right)}$ pode ser estendido ${ }^{8}$ a um estado em $S_{C\left(S^{\mathbb{Z}^{d+1}}\right)}$ o qual é um conjunto compacto ${ }^{9}$ na topologia fraca-*, e assim existe o $\lim _{\Lambda_{\alpha}} \mu_{\Lambda_{\alpha}}=: \mu \in S_{C\left(S^{\mathbb{Z}^{d+1}}\right)}$ de uma sub-rede de $\left\{\mu_{\Lambda}\right\}_{\Lambda \in P F\left(\mathbb{Z}^{d+1}\right)}$ e define um estado em $C\left(S^{\mathbb{Z}^{d+1}}\right)$ que está localmente especificado a partir dos $\mu_{\Lambda_{\alpha}}$.

No seguinte vamos supor $S=\{1,-1\}$. Para cada $\Lambda \in P F\left(\mathbb{Z}^{d+1}\right)$ definimos o

\footnotetext{
${ }^{5}[97]$, [56] p. 204.

${ }^{6} \mathrm{Cf} .2 \cdot 1 \cdot 1(\mathrm{~d})$.

${ }^{7}$ Veja-se $[29]$ p. 7 , e as referências aí citadas.

${ }^{8}$ Veja-se [23], 5.16 na p. 252.

${ }^{9}$ Ibidem, 5.15 na p. 252.
} 
conjunto de vizinhos próximos ou ligações em $\Lambda$ como:

$$
\mathcal{L}(\Lambda):=\{\{x, y\}: x \in \Lambda, y \in \Lambda \text { e }|x-y|=1\} .
$$

Para estudar o modelo de Ising na região de altas temperaturas resulta útil definir o seguinte estado:

$$
\mu_{\Lambda}(f):=\frac{\sum_{\sigma \in S^{\Lambda}} f(\sigma) \mathrm{e}^{\beta \sum_{L \in \mathcal{L}(\Lambda)} j_{L} p_{L}(\sigma)}}{\sum_{\sigma \in S^{\Lambda}} \mathrm{e}^{\beta \sum_{L \in \mathcal{L}(\Lambda)} j_{L} p_{L}(\sigma)}} \forall f \in C\left(S^{\Lambda}\right),
$$

onde:

$$
j_{L}:= \begin{cases}j & \text { se } L=\{(n, \mathrm{x}),(n, \mathrm{y})\} \\ j_{m} & \text { se } L=\{(m, \mathrm{x}),(m+1, \mathrm{x})\}\end{cases}
$$

sendo $j, j_{m}$ constantes positivas. A primeira delas mede o acoplamento entre vizinhos próximos jazendo ambos num mesmo hiperplano definido por $x_{0}=$ constante $=n$, no entanto as segundas, fazem o mesmo com vizinhos próximos que jazem em dois de tais hiperplanos distintos. Observe que:

$$
\mu_{\Lambda}(f)=\frac{\operatorname{tr}\left(D(f) D\left(\mathrm{e}^{-\beta H_{\Lambda}}\right)\right)}{\operatorname{tr}\left(D\left(\mathrm{e}^{-\beta H_{\Lambda}}\right)\right)}
$$

onde:

$$
H_{\Lambda}(\sigma):=-\sum_{L \in \mathcal{L}(\Lambda)} j_{L} p_{L}(\sigma)
$$

e $D: C\left(S^{\Lambda}\right) \longmapsto D_{2^{|\Lambda|}}(\mathbb{C}) \operatorname{com} D(f):=\operatorname{diag}\left(f\left(\sigma_{1}\right), \ldots, f\left(\sigma_{2^{|\Lambda|}}\right)\right) .{ }^{10}$ Claramente, $\mu_{\Lambda} \in S_{C\left(S^{\Lambda}\right)}$. O modelo de Ising (ferromagnético, sem campo magnético, em $d+1$ dimensões) será, no presente contexto, o modelo de TQC definido pela família de estados locais acima com $0<j=j_{m} \forall m \in \mathbb{Z}$.

4.2.1 Observação: O estado acima não é meramente positivo senão que satisfaz as seguintes desigualdades de Griffiths: ${ }^{11}$

(a) $0 \leqslant \mu_{\Lambda}\left(p_{A}\right)$

(b) $0 \leqslant \mu_{\Lambda}\left(p_{A} p_{B}\right)-\mu_{\Lambda}\left(p_{A}\right) \mu_{\Lambda}\left(p_{B}\right)$

Aqui supõe-se que os $j_{L}$ são reais. Observe-se também que:

\footnotetext{
${ }^{10}$ Cf. 2.1.1(b) na p. 17.

${ }^{11}$ Veja-se [36] pp. 57-59.
} 
(c) Fixado $A \subseteq \Lambda$, para cada $L \in \mathcal{L}(\Lambda)$ a aplicação $j_{L} \longmapsto \mu_{\Lambda}\left(p_{A}\right)$ é monótona não decrescente. Com efeito,

$$
\frac{d \mu_{\Lambda}\left(p_{A}\right)}{d j_{L}}=\beta\left(\mu_{\Lambda}\left(p_{A} p_{L}\right)-\mu_{\Lambda}\left(p_{A}\right) \mu_{\Lambda}\left(p_{L}\right)\right) \geqslant 0,
$$

pelo ponto (b) anterior.

(d) $\left|\mu_{\Lambda}\left(p_{A}\right)\right| \leqslant 1$.

Define-se:

$$
\mathcal{S}_{n}^{\Lambda}\left(x_{1}, \ldots, x_{n}\right):=\mu_{\Lambda}\left(p_{x_{1}} \cdots p_{x_{n}}\right)=\mu_{\Lambda}\left(p_{\left\{x_{1}, \ldots, x_{n}\right\}}\right)
$$

4.2.2 Observação: Se $\Lambda_{1} \subseteq \Lambda_{2}$, então definindo $j_{L}=0$ se $L \in \mathcal{L}\left(\Lambda_{2}\right) \backslash \mathcal{L}\left(\Lambda_{1}\right)$ tem-se que:

$$
\mu_{\Lambda_{1}}\left(p_{A}\right)=\frac{\sum_{\sigma \in S^{\Lambda}} p_{A}(\sigma) \mathrm{e}^{\beta \sum_{L \in \mathcal{L}\left(\Lambda_{1}\right)} j_{L} p_{L}(\sigma)}}{\sum_{\sigma \in S^{\Lambda}} \mathrm{e}^{\beta \sum_{L \in \mathcal{L}\left(\Lambda_{1}\right)} j_{L} p_{L}(\sigma)}}=\frac{\sum_{\sigma \in S^{\Lambda}} p_{A}(\sigma) \mathrm{e}^{\beta \sum_{L \in \mathcal{L}\left(\Lambda_{2}\right)} j_{L} p_{L}(\sigma)}}{\sum_{\sigma \in S^{\Lambda}} \mathrm{e}^{\beta \sum_{L \in \mathcal{L}\left(\Lambda_{2}\right)} j_{L} p_{L}(\sigma)}}
$$

Por outro lado (desta vez sem redefinir os $j_{L}$ ):

$$
\mu_{\Lambda_{2}}\left(p_{A}\right)=\frac{\sum_{\sigma \in S^{\Lambda}} p_{A}(\sigma) \mathrm{e}^{\beta \sum_{L \in \mathcal{L}\left(\Lambda_{2}\right)} j_{L} p_{L}(\sigma)}}{\sum_{\sigma \in S^{\Lambda}} \mathrm{e}^{\beta \sum_{L \in \mathcal{L}\left(\Lambda_{2}\right)} j_{L} p_{L}(\sigma)}}
$$

Portanto, fixado $L \in \mathcal{L}\left(\Lambda_{2}\right)$, tem-se $\mu_{\Lambda_{1}}\left(p_{A}\right) \leqslant \mu_{\Lambda_{2}}\left(p_{A}\right)$ por 4.2 .1 (c) e a redefinição dos $j_{L}$ como acima no primeiro caso. Portanto, de 4.2.1(d) segue que existe $\lim _{\Lambda} \mu_{\Lambda}\left(p_{A}\right)$.

Em particular, existem:

$$
\mathcal{S}_{n}\left(x_{1}, \ldots, x_{n}\right):=\lim _{\Lambda} \mathcal{S}_{n}^{\Lambda}\left(x_{1}, \ldots, x_{n}\right)
$$

que serão, por definição, as funções de $n$ pontos para o modelo de Ising. O limite no lado direito acima denota o limite no conjunto dirigido considerado em 2.1.1(d), denotado alternativamente como $\Lambda \uparrow \mathbb{Z}^{d+1}$. Observe que este limite é pontual.

4.2.3 Observação: Da expressão:

$$
\mathcal{S}_{n}^{\Lambda}\left(x_{1}, \ldots, x_{n}\right)=\frac{\sum_{\sigma \in S^{\Lambda}} \sigma\left(x_{1}\right) \cdots \sigma\left(x_{n}\right) \prod_{L \in \mathcal{L}(\Lambda)} \mathrm{e}^{\beta j_{L} p_{L}(\sigma)}}{\sum_{\sigma \in S^{\Lambda}} \prod_{L \in \mathcal{L}(\Lambda)} \mathrm{e}^{\beta j_{L} p_{L}(\sigma)}}
$$


segue que $\mathcal{S}_{2 n-1}^{\Lambda}$ (e portanto $\mathcal{S}_{2 n-1}$ ) são nulas $\forall n \in \mathbb{N}$, pois na somatória a contribuição de uma dada $\sigma$ será cancelada pela de $-\sigma$ dado que o exponente é invariante pela substituição de $\sigma$ por $-\sigma$.

No seguinte vamos supor que os $j_{m}$ admitem também valores complexos.

4.2.4 Lema: Seja $g_{m}:=\tanh \left(\beta j_{m}\right)$. Então:

(a)

$$
\begin{aligned}
\partial_{g_{m}} \mathcal{S}_{n}\left(x_{1}, \ldots, x_{n}\right)=\frac{1}{1-g_{m}^{2}}\left[\sum_{\mathbf{x} \in \mathbb{Z}^{d}} \mathcal{S}_{n+2}\left(x_{1}, \ldots, x_{n},(m, \mathbf{x}),(m+1, \mathbf{x})\right)\right. \\
\\
\left.\quad-\mathcal{S}_{n}\left(x_{1}, \ldots, x_{n}\right) \sum_{\mathbf{x} \in \mathbb{Z}^{d}} \mathcal{S}_{2}((m, \mathbf{x}),(m+1, \mathbf{x}))\right] .
\end{aligned}
$$

(b)

$$
\begin{aligned}
& \partial_{g_{m}}^{2} \mathcal{S}_{n}\left(x_{1}, \ldots, x_{n}\right) \\
& =\frac{2 g_{m}}{\left(1-g_{m}^{2}\right)^{2}}\left[\sum_{\mathrm{x} \in \mathbb{Z}^{d}} \mathcal{S}_{n+2}\left(x_{1}, \ldots, x_{n},(m, \mathrm{x}),(m+1, \mathrm{x})\right)\right. \\
& \left.-\mathcal{S}_{n}\left(x_{1}, \ldots, x_{n}\right) \sum_{\mathbf{x} \in \mathbb{Z}^{d}} \mathcal{S}_{2}((m, \mathrm{x}),(m+1, \mathrm{x}))\right] \\
& +\frac{1}{1-g_{m}^{2}}\left[\sum_{\mathbf{x}, \mathbf{y} \in \mathbb{Z}^{d}} \mathcal{S}_{n+4}\left(x_{1}, \ldots, x_{n},(m, \mathrm{x}),(m+1, \mathrm{x}),(m, \mathrm{y}),(m+1, \mathrm{y})\right)\right. \\
& -2\left(\sum_{\mathbf{x} \in \mathbb{Z}^{d}} \mathcal{S}_{n+2}\left(x_{1}, \ldots, x_{n},(m, \mathbf{x}),(m+1, \mathbf{x})\right)\right)\left(\sum_{\mathbf{y} \in \mathbb{Z}^{d}} \mathcal{S}_{2}((m, \mathrm{y}),(m+1, \mathrm{y}))\right) \\
& +2 \mathcal{S}_{n}\left(x_{1}, \ldots, x_{n}\right)\left(\sum_{\mathbf{x} \in \mathbb{Z}^{d}} \mathcal{S}_{2}((m, \mathrm{x}),(m+1, \mathrm{x}))\right)\left(\sum_{\mathbf{y} \in \mathbb{Z}^{d}} \mathcal{S}_{2}((m, \mathrm{y}),(m+1, \mathrm{y}))\right) \\
& \left.-\mathcal{S}_{n}\left(x_{1}, \ldots, x_{n}\right) \sum_{\mathbf{x}, \mathbf{y} \in \mathbb{Z}^{d}} \mathcal{S}_{4}((m, \mathrm{x}),(m+1, \mathrm{x}),(m, \mathrm{y}),(m+1, \mathrm{y}))\right] \text {. }
\end{aligned}
$$


Demonstraçấo: Para a parte (a), observe que $\partial_{g_{m}} \mathcal{S}_{n}=\partial_{j_{m}} \mathcal{S}_{n} \frac{\mathrm{d} j_{m}}{\mathrm{~d} g_{m}}$. Agora, da definição de $g_{m}$ temos que $j_{m}=\frac{1}{\beta} \operatorname{arctanh} g_{m}$, de onde:

$$
\frac{\mathrm{d} j_{m}}{\mathrm{~d} g_{m}}=\frac{\beta^{-1}}{1-g_{m}^{2}}
$$

Por outro lado, denotando por $Z$ o denominador na identidade de 4.2.3, temos:

$$
\begin{aligned}
& \partial_{j_{m}} \mathcal{S}_{n}\left(x_{1}, \ldots, x_{n}\right) \\
& =\frac{\sum_{\sigma \in S^{\Lambda}} \sigma\left(x_{1}\right) \cdots \sigma\left(x_{n}\right)\left(\beta \sum_{\mathbf{x} \in \mathbb{Z}^{d}} \sigma(m, \mathbf{x}) \sigma(m+1, \mathrm{x})\right) \prod_{L \in \mathcal{L}(\Lambda)} \mathrm{e}^{\beta j_{L} p_{L}(\sigma)}}{Z} \\
& \quad-\frac{Z \mathcal{S}_{n}\left(x_{1}, \ldots, x_{n}\right) \sum_{\sigma \in S^{\Lambda}} \beta \sum_{\mathbf{x} \in \mathbb{Z}^{d}} \sigma(m, \mathrm{x}) \sigma(m+1, \mathrm{x}) \prod_{L \in \mathcal{L}(\Lambda)} \mathrm{e}^{\beta j_{L} p_{L}(\sigma)}}{Z^{2}} \\
& =\beta \sum_{\mathbf{x} \in \mathbb{Z}^{d}} \mathcal{S}_{n+2}\left(x_{1}, \ldots, x_{n},(m, \mathbf{x}),(m+1, \mathbf{x})\right) \\
& \quad-\mathcal{S}_{n}\left(x_{1}, \ldots, x_{n}\right) \mathcal{S}_{2}((m, \mathrm{x}),(m+1, \mathbf{x})) .
\end{aligned}
$$

Para a parte (b), basta derivar a expressão da parte (a) anterior, usando-a também para substituir as derivadas resultantes.

\subsection{Expansão em Polímeros na Região de Altas Temperaturas}

Começamos introduzindo algumas definições. Se $M \in P(\mathcal{L}(\Lambda))$ define-se:

$$
\chi_{M}:=\{x \in \Lambda: \exists y \in \Lambda:\{x, y\} \in M\},
$$

e para cada $x \in \chi_{M}$ define-se:

$$
M_{x}:=\{L \in M: x \in L\} .
$$

Por outro lado:

$$
\mathcal{M}:=\left\{M \in P(\mathcal{L}(\Lambda)):(-1)^{\left|M_{x}\right|}=1 \forall x \in \chi_{M}\right\} .
$$

Ou seja, cada $M \in \mathcal{M}$ é um conjunto de vizinhos próximos tais que cada um dos seus pontos (em $\chi_{M}$ ) é integrante de um número par de vizinhos. Agora, para cada par de pontos $i, j \in \mathbb{Z}^{d+1}$ define-se:

$$
\begin{array}{r}
\operatorname{conn}(i, j):=\left\{M \in P(\mathcal{L}(\Lambda)): M \text { é conexo, }(-1)^{\left|M_{i}\right|}=-1=(-1)^{\left|M_{j}\right|}\right. \\
\text { e } \left.(-1)^{\left|M_{x}\right|}=1 \forall x \in \chi_{M} \backslash\{i, j\}\right\} .
\end{array}
$$


Aqui, $M$ conexo significa que para cada par $x, y \in \chi_{M}$ existe uma família de vizinhos próximos $\left\{L_{i}\right\}_{i=1}^{k} \subseteq M$ tal que $x \in \chi_{L_{1}}, y \in \chi_{L_{k}}$ e $\chi_{L_{i}} \cap \chi_{L_{i+1}} \neq \emptyset \forall i$. Os $M \in \operatorname{conn}(i, j)$ serão chamados de caminhos que conectam $i$ com $j$. Analogamente, um elemento de $\operatorname{conn}\left(i_{1}, \ldots, i_{2 n}\right)$ será, por definição, uma família de $n$ caminhos que unem pares disjuntos de pontos em $\left\{i_{1}, \ldots, i_{2 n}\right\}$ :

$$
\begin{aligned}
& \operatorname{conn}\left(i_{1}, \ldots, i_{2 n}\right):=\left\{\left\{M^{k}\right\}_{k=1}^{n}: M^{k} \in \operatorname{conn}\left(i_{k_{1}}, i_{k_{2}}\right), \quad(-1)\left|\bigcup_{k=1}^{n} M_{i_{j}}^{k}\right|=-1\right. \\
& \left.\forall j=1, \ldots, 2 n \text { e }(-1)^{\left|\cup_{k=1}^{n} M_{x}^{k}\right|}=1 \quad \forall x \in\left(\cup_{k=1}^{n} \chi_{M^{k}}\right) \backslash\left\{i_{1}, \ldots, i_{2 n}\right\}\right\}_{P \in \mathcal{P}} .
\end{aligned}
$$

Aqui $\mathcal{P}$ denota o conjunto de partições do conjunto $\{1, \ldots, 2 n\}$ em $n$ pares. Não será necessário considerar um número ímpar de pontos por causa de 4.2.3.

O índice $\Lambda$ é suprimido em $\chi_{M}, M_{x}, \mathcal{M}$ e conn, mas apenas para facilitar a notação. Partindo da identidade em 4.2.3 temos:

$$
\begin{gathered}
\mathcal{S}_{2 n}^{\Lambda}\left(i_{1}, \ldots, i_{2 n}\right)=\frac{\sum_{\sigma \in S^{\Lambda}} \sigma\left(i_{1}\right) \cdots \sigma\left(i_{2 n}\right) \prod_{L \in \mathcal{L}(\Lambda)}\left(1+g_{L} p_{L}(\sigma)\right)}{\sum_{\sigma \in S^{\Lambda}} \prod_{L \in \mathcal{L}(\Lambda)}\left(1+g_{L} p_{L}(\sigma)\right)} \\
=\frac{\sum_{M \in P(\mathcal{L}(\Lambda))}\left(\prod_{L \in M} g_{L}\right)\left(\sum_{\sigma} \sigma\left(i_{1}\right) \cdots \sigma\left(i_{2 n}\right) \prod_{L \in M} p_{L}(\sigma)\right)}{\sum_{M \in P(\mathcal{L}(\Lambda))}\left(\prod_{L \in M} g_{L}\right)\left(\sum_{\sigma} \prod_{L \in M} p_{L}(\sigma)\right)} \\
=\frac{\sum_{M \in P(\mathcal{L}(\Lambda))}\left(\prod_{L \in M} g_{L}\right)\left(\sum_{\sigma} \sigma\left(i_{1}\right) \cdots \sigma\left(i_{2 n}\right) \prod_{x \in \chi_{M}} \sigma(x)^{\left|M_{x}\right|}\right)}{\sum_{M \in P(\mathcal{L}(\Lambda))}\left(\prod_{L \in M} g_{L}\right)\left(\sum_{\sigma} \prod_{x \in \chi_{M}} \sigma(x)^{\left|M_{x}\right|}\right)},
\end{gathered}
$$

onde $g_{L}:=\tanh \left(\beta j_{L}\right)$, com a convenção que se $M=\emptyset$ então $\prod_{L \in M} \cdots=1$. Observe que na somatória no denominador apenas têm contribuição não nula os $M \in \mathcal{M}$ pois, caso contrário, na somatória sobre as configurações a contribuição de uma dada $\sigma$ é cancelada pela de $-\sigma$ ou pela de uma outra configuração obtida a partir de $\sigma$ pela inversão de spins em alguns sítios. Em caso que $M \in \mathcal{M}$, a produtória vale 1 e assim a somatória sobre as configurações vale $\left|S^{\Lambda}\right|=2^{|\Lambda|}$. Com um raciocínio análogo, no numerador contribuem apenas os $M$ da forma $M=M_{0} \cup M_{1}$ com $M_{0} \in \operatorname{conn}\left(i_{1}, \ldots, i_{2 n}\right), M_{1} \in \mathcal{M}$ e $\chi_{M_{0}} \cap \chi_{M_{1}}=\emptyset$, em cujo caso a somatória sobre todas as configurações também vale $2^{|\Lambda|}$. Portanto:

$$
\mathcal{S}_{n}^{\Lambda}\left(i_{1}, \ldots, i_{2 n}\right)=\sum_{M_{0} \in \operatorname{conn}\left(i_{1}, \ldots, i_{2 n}\right)}\left(\prod_{L \in M_{0}} g_{L}\right)\left(\frac{\sum_{\substack{M \in \mathcal{M} \\ \chi_{M} \cap \chi_{M_{0}}=\emptyset}} \prod_{L \in M} g_{L}}{\sum_{M \in \mathcal{M}} \prod_{L \in M} g_{L}}\right)
$$

Seja:

$$
G:=\{M \in \mathcal{M}: M \text { é conexo }\}
$$


Como $G \subseteq \mathcal{M} \subseteq P(\mathcal{L}(\Lambda))$, e portanto $r:=|G| \leqslant|P(\mathcal{L}(\Lambda))|=2^{|\mathcal{L}(\Lambda)|} \leqslant 2^{2(d+1)|\Lambda|}<$ $\infty$, podemos supor $G=\left\{\gamma_{1}, \ldots, \gamma_{r}\right\}$. Os elementos de $G$ são chamados de polímeros. Definimos:

$$
\begin{aligned}
\mathcal{G}(\Lambda) & :=\{\Gamma: G \longmapsto\{0,1\}\} \\
\mathcal{G}^{\prime}(\Lambda) & :=\{\Gamma: G \longmapsto \mathbb{N} \cup\{0\}\}
\end{aligned}
$$

O conjunto $\mathcal{G}(\Lambda)$ é basicamente $P(G)$. A função $\Gamma$ pode ser pensada como "escolhendo" os subconjuntos de $G$ que vão fazer parte de um dado elemento em $P(G)$, $v . g$., se $\Gamma(\gamma)=1$ ou 0 significa que $\gamma \in G$ foi escolhido ou não, respectivamente. A função $\Gamma=0$ corresponde ao conjunto $\emptyset \in P(G)$. O conjunto $\mathcal{G}^{\prime}(\Lambda)$ pode ser pensado como $P(G)$ mas onde cada conjunto pode ser escolhido várias vezes. Em $P(\mathcal{L}(\Lambda))$ definimos a relação $\sim$ por:

$$
M \sim N \Longleftrightarrow \chi_{M} \cap \chi_{N}=\emptyset
$$

em cujo caso se diz que $M$ e $N$ são compatíveis. Definimos agora:

$$
\mathcal{G}^{c}(\Lambda):=\left\{\Gamma \in \mathcal{G}(\Lambda): \gamma, \gamma^{\prime} \in \operatorname{supp} \Gamma \wedge \gamma \neq \gamma^{\prime} \Longrightarrow \gamma \sim \gamma^{\prime}\right\}
$$

Os elementos de $\mathcal{G}^{c}(\Lambda)$ são subconjuntos de $G$ integrados por polímeros mutuamente compatíveis (i.e., $\chi_{\gamma}$ e $\chi_{\gamma^{\prime}}$ disjuntos dois a dois). Como todo $M \in \mathcal{M}$ pode ser expressado como união disjunta de conexos, definindo:

$$
\begin{aligned}
\mu(\gamma) & :=\prod_{L \in \gamma} g_{L} \\
\mu^{\Gamma} & :=\prod_{L \in \gamma} \mu(\gamma)^{\Gamma(\gamma)},
\end{aligned}
$$

o denominador do segundo fator na somatória em (4.3.1) pode ser expressado como:

$$
\sum_{M \in \mathcal{M}} \prod_{L \in M} g_{L}=\sum_{\Gamma \in \mathcal{G}^{c}(\Lambda)} \prod_{\gamma \in G}\left(\prod_{L \in \gamma} g_{L}\right)^{\Gamma(\gamma)}=\sum_{\Gamma \in \mathcal{G}^{c}(\Lambda)} \mu^{\Gamma},
$$

de onde:

$$
\begin{aligned}
\ln \sum_{\Gamma \in \mathcal{G}^{c}(\Lambda)} \mu^{\Gamma}=\ln \left(1+\sum_{\substack{\Gamma \in \mathcal{G}^{c}(\Lambda) \\
\Gamma \neq 0}} \mu^{\Gamma}\right)=\sum_{n=0}^{\infty} \frac{(-1)^{n+1}}{n}\left(\sum_{\substack{\Gamma \in \mathcal{G}^{c}(\Lambda) \\
\Gamma \neq 0}} \mu^{\Gamma}\right)^{n} \\
=\sum_{n=0}^{\infty} \frac{(-1)^{n+1}}{n} \sum_{\substack{\Gamma_{i} \in \mathcal{G}^{c}(\Lambda) \\
\Gamma \neq 0}} \mu^{\Gamma_{1}+\cdots+\Gamma_{n}}=\sum_{\Gamma \in \mathcal{G}^{\prime}(\Lambda)} c_{\Gamma} \mu^{\Gamma}
\end{aligned}
$$


pois $\Gamma_{1}+\cdots+\Gamma_{n} \in \mathcal{G}^{\prime}(\Lambda)$, sendo:

$$
c_{\Gamma}:=\sum_{n=0}^{\infty} \frac{(-1)^{n+1}}{n} N_{n}(\Gamma),
$$

onde $N_{n}(\Gamma)$ é o número de maneiras diferentes de escrever $\Gamma \in \mathcal{G}^{\prime}(\Lambda)$ na forma $\Gamma=\Gamma_{1}+\cdots+\Gamma_{n} \operatorname{com} \Gamma_{i} \in \mathcal{G}^{c}(\Lambda), \Gamma_{i} \neq 0 \forall i$. Analogamente, se para cada $M \in P(\mathcal{L}(\Lambda))$ e para cada $\Gamma \in \mathcal{G}^{c}(\Lambda)$ ou $\mathcal{G}^{\prime}(\Lambda)$ denotamos $\Gamma \sim M$ se e somente se $\gamma \sim M \forall \gamma \in \operatorname{supp} \Gamma$, ou $\Gamma \nsim M$ caso contrário, temos para o logaritmo natural do numerador do segundo fator na somatória em (4.3.1):

$$
\ln \sum_{\substack{M \in \mathcal{M} \\ \chi_{M} \cap \chi_{M_{0}}=\emptyset}} \prod_{L \in M} g_{L}=\ln \sum_{\substack{\Gamma \in \mathcal{G}^{c}(\Lambda) \\ \Gamma \sim M_{0}}} \mu^{\Gamma}=\sum_{\substack{\Gamma \in \mathcal{G}^{\prime}(\Lambda) \\ \Gamma \sim M_{0}}} c_{\Gamma} \mu^{\Gamma} .
$$

Introduzindo (4.3.2) e (4.3.3) em (4.3.1) temos:

$$
\begin{array}{r}
\mathcal{S}_{2 n}^{\Lambda}\left(i_{1}, \ldots, i_{2 n}\right)=\sum_{M_{0} \in \operatorname{conn}\left(i_{1}, \ldots, i_{2 n}\right)}\left(\prod_{L \in M_{0}} g_{L}\right) \exp \left(\sum_{\Gamma \in \mathcal{G}^{\prime}(\Lambda)} c_{\Gamma} \mu^{\Gamma}-\sum_{\substack{\Gamma \in \mathcal{G}^{c}(\Lambda) \\
\Gamma \sim M_{0}}} c_{\Gamma} \mu^{\Gamma}\right) \\
=\sum_{M \in \operatorname{conn}\left(i_{1}, \ldots, i_{2 n}\right)}\left(\prod_{L \in M} g_{L}\right) \exp \sum_{\substack{\Gamma \in \mathcal{G}^{\prime}(\Lambda) \\
\Gamma \nsim M}} c_{\Gamma} \mu^{\Gamma} .
\end{array}
$$

A expressão acima é chamada expansão em polímeros para função de $2 n$ pontos. Definindo:

$$
|\mu|:=\sup _{\gamma \in G}|\mu(\gamma)|^{1 /|\gamma|}
$$

(observe que $|\mu|=|g|$ no modelo de Ising), se pode provar que a série no exponente do segundo fator em (4.3.4) é absolutamente convergente se $|\mu|$ é suficientemente pequeno. Mais especificamente:

\subsubsection{Lema:}

$$
\exists \mu_{0}>0:|\mu|<\mu_{0} \Longrightarrow \sum_{\substack{\Gamma \in \mathcal{G}^{\prime}(\Lambda) \\ \Gamma \nsim M}}\left|c_{\Gamma}\right|\left|\mu^{\Gamma}\right| \leqslant c|M|
$$

onde $\mu_{0}$ e c são constantes independentes de $\Lambda$.

Referências: [9]; [31], Appendix A.1; [91]. 
Em particular, cada $\mathcal{S}_{2 n}^{\Lambda}\left(i_{1}, \ldots, i_{2 n}\right)$ é função analítica de cada $g, g_{m}$ numa vizinhança da origem. Com efeito, usando 4.2.1(a), 4.3.4 e a desigualdade em 4.3.1 acima, temos:

$$
\begin{aligned}
& \mathcal{S}_{2 n}^{\Lambda}\left(i_{1}, \ldots, i_{2 n}\right) \leqslant \sum_{M \in \operatorname{conn}\left(i_{1}, \ldots, i_{2 n}\right)}\left(\prod_{L \in M}\left|g_{L}\right|\right) \mathrm{e}^{c|M|} \leqslant \sum_{M \in \operatorname{conn}\left(i_{1}, \ldots, i_{2 n}\right)}\left(g_{\infty} \mathrm{e}^{c}\right)^{|M|} \\
& \leqslant \sum_{P \in \mathcal{P}} \sum_{\substack{M \text { caminho } \\
\text { partindo de } i_{k_{1}} \\
|M| \geqslant \operatorname{dist}\left(i_{k_{1}}, i_{k_{2}}\right)}}\left(g_{\infty} \mathrm{e}^{c}\right)^{|M|}=\frac{(2 n) !}{2^{n} n !} n \sum_{|M|=a}^{\infty}\left(2(d+1) g_{\infty} \mathrm{e}^{c}\right)^{|M|} \\
& =\frac{(2 n) !}{2^{n}(n-1) !} \frac{\left(2(d+1) g_{\infty} \mathrm{e}^{c}\right)^{a}}{1-\left(2(d+1) g_{\infty} \mathrm{e}^{c}\right)}
\end{aligned}
$$

onde $g_{\infty}:=\sup \left\{|g|,\left|g_{m}\right|: m \in \mathbb{Z}\right\}, a:=\max _{\mathcal{P}} \max _{k} \operatorname{dist}\left(i_{k_{1}}, i_{k_{2}}\right)$. Como o último membro não depende de $\Lambda$, temos que $\mathcal{S}_{2 n}\left(i_{1}, \ldots, i_{2 n}\right)$ é limite de uma rede convergente de funções analíticas de cada $g, g_{m}$ numa vizinhança da origem.

Definindo:

$$
L_{m}:=\Lambda_{0, m} \backslash \Lambda_{0, m+1}=\left\{\left(x_{0}, x_{1}, \ldots, x_{d}\right) \in \mathbb{Z}^{d+1}: x_{0}=m\right\},
$$

a expansão em polímeros permite provar o seguinte

4.3.2 Lema: Suponha-se que o hiperplano $L_{m}$ separa o conjunto de sítios $\left\{i_{1}, \ldots, i_{2 n}\right\}$ em dois conjuntos, digamos $\left\{i_{k_{1}}, \ldots, i_{k_{r}}\right\}$ e $\left\{i_{k_{r+1}}, \ldots, i_{k_{2 n}}\right\}$ (admitindo-se que um deles seja eventualmente vazio), no sentido que:

$$
\left\{i_{k_{1}}, \ldots, i_{k_{r}}\right\} \subseteq \Lambda_{0, m+1}^{c} \quad \text { e } \quad\left\{i_{k_{r+1}}, \ldots, i_{k_{2 n}}\right\} \subseteq \Lambda_{0, m+1}
$$

Então:

(a) Se $r$ é par, digamos $r=2 l$, então $\left.\mathcal{S}_{2 n}\left(i_{1}, \ldots, i_{2 n}\right)\right|_{g_{m}=0}$ fatora na seguinte forma:

$$
\left.\mathcal{S}_{2 n}\left(i_{1}, \ldots, i_{2 n}\right)\right|_{g_{m}=0}=\left.\left.\mathcal{S}_{2 l}\left(i_{k_{1}}, \ldots, i_{k_{2 l}}\right)\right|_{g_{m}=0} \mathcal{S}_{2 n-2 l}\left(i_{k_{2 l+1}}, \ldots, i_{2 n}\right)\right|_{g_{m}=0} .
$$

Se $n \in \mathbb{N} \cup\{0\}$, tem-se:

(b) Se r é ímpar, então $\left.\partial_{g_{m}}^{2 n} \mathcal{S}_{2 n}\left(i_{1}, \ldots, i_{2 n}\right)\right|_{g_{m}=0}=0$.

(c) Se r é par, então $\left.\partial_{g_{m}}^{2 n+1} \mathcal{S}_{2 n}\left(i_{1}, \ldots, i_{2 n}\right)\right|_{g_{m}=0}=0$. 
Demonstração: Observe-se que ao avaliar $\mathcal{S}_{2 n}\left(i_{1}, \ldots, i_{2 n}\right)$ em $g_{m}=0$, na somatória em (4.3.4) apenas têm contribuição não nula os caminhos $M \in \operatorname{conn}\left(i_{1}, \ldots, i_{2 n}\right)$ que não dependem de $g_{m}$.

No caso de existir uma quantidade par de sítios a cada lado de $L_{m}$, tais caminhos serão os que conectam pares de sítios que jazem do mesmo lado do hiperplano e sem atravessá-lo (os caminhos que não fazem isso devem atravessar $L_{m}$, em cujo caso aparece $g_{m}$ pelo menos uma vez como fator, dando contribuição nula ao avaliar em zero). Neste caso a função de $2 n$ pontos fatoriza, pois os polímeros que intervêm na somatória no exponente do segundo fator na somatória em (4.3.4) resultam incompatíveis com pelo menos um dos caminhos que não atravessam o hiperplano (ou então, o polímero atravessa $L_{m}$ em cujo caso depende de $g_{m}$ (na verdade de $g_{m}^{2}$ ou potências pares superiores, pela conexidade) e assim ao avaliar em zero sua contribuição no exponente é nula, ou seja, contribui com um 1 como fator). Isto prova (a).

Derivando a expressão (4.3.4) obtem-se:

$$
\begin{aligned}
\partial_{g_{m}} \mathcal{S}_{2 n}^{\Lambda}\left(i_{1}, \ldots, i_{2 n}\right)= & \sum_{M \in \operatorname{conn}\left(i_{1}, \ldots, i_{2 n}\right)} \partial_{g_{m}}\left(\prod_{L \in M} g_{L}\right) \exp \sum_{\substack{\Gamma \in \mathcal{G}^{\prime}(\Lambda) \\
\Gamma \nsim M}} c_{\Gamma} \mu^{\Gamma} \\
& +\sum_{M \in \operatorname{conn}\left(i_{1}, \ldots, i_{2 n}\right)}\left(\prod_{L \in M} g_{L}\right) \partial_{g_{m}} \exp \sum_{\substack{\Gamma \in \mathcal{G}^{\prime}(\Lambda) \\
\Gamma \nsim M}} c_{\Gamma} \mu^{\Gamma} .
\end{aligned}
$$

As derivadas de ordem superior se obtêm derivando sucessivamente esta identidade. Para a parte (b), observe-se que as derivadas pares têm a forma:

$$
\partial_{g_{m}}^{2 n} \mathcal{S}_{2 n}\left(i_{1}, \ldots, i_{2 n}\right)=\sum_{M \in \operatorname{conn}\left(i_{1}, \ldots, i_{2 n}\right)} A_{1}+A_{2} .
$$

Aqui, $A_{1}$ é uma soma de termos da forma:

$$
\text { const } \partial_{g_{m}}^{(\mathrm{par})}\left(\prod_{L \in M} g_{L}\right) \partial_{g_{m}}^{(\mathrm{par})} \exp \sum_{\substack{\Gamma \in \mathcal{G}^{\prime}(\Lambda) \\ \Gamma \nsim M}} c_{\Gamma} \mu^{\Gamma} .
$$

Em caso de existir uma quantidade ímpar de sítios a cada lado de $L_{m}$, devem existir caminhos unindo pares de sítios em lados diferentes do hiperplano. Mais ainda, tais caminhos neste caso devem atravessar o hiperplano uma quantidade ímpar de vezes e deve existir uma quantidade ímpar de tais caminhos. Isto faz com que o fator entre parênteses acima dependa de potências ímpares de $g_{m}$ e portanto a sua derivada se anula ao avaliar em zero, de onde $A_{1}=0$. 
Por outro lado, $A_{2}$ é uma soma de termos da forma:

$$
\text { const } \partial_{g_{m}}^{\text {(impar) }}\left(\prod_{L \in M} g_{L}\right) \partial_{g_{m}}^{\text {(impar) }} \exp \sum_{\substack{\Gamma \in \mathcal{G}^{\prime}(\Lambda) \\ \Gamma \nsim M}} c_{\Gamma} \mu^{\Gamma},
$$

que também são nulos, pois o exponente depende de potências pares de $g_{m}$ pela conexidade dos polímeros.

Para a parte (c), temos analogamente:

$$
\partial_{g_{m}}^{2 n+1} \mathcal{S}_{2 n}\left(i_{1}, \ldots, i_{2 n}\right)=\sum_{M \in \operatorname{conn}\left(i_{1}, \ldots, i_{2 n}\right)} B_{1}+B_{2} .
$$

Aqui, $B_{1}$ é uma soma de termos da forma:

$$
\text { const } \partial_{g_{m}}^{\text {(ímpar) }}\left(\prod_{L \in M} g_{L}\right) \partial_{g_{m}}^{(\text {par })} \exp \sum_{\substack{\Gamma \in \mathcal{G}^{\prime}(\Lambda) \\ \Gamma \nsim M}} c_{\Gamma} \mu^{\Gamma} .
$$

Os caminhos que não atravessam o hiperplano não dependem de $g_{m}$ e portanto têm contribuição nula ao derivar. Os que atravessam $L_{m}$ unindo pontos que ficam do mesmo lado, devem atravessá-lo uma quantidade par de vezes. Os que unem pontos em lados diferentes o atravessam uma quantidade ímpar de vezes, mas se $r$ é par sempre existirá neste caso uma quantidade par de tais caminhos. Assim, o fator entre parênteses acima depende de potências pares de $g_{m}$ e portanto a sua derivada se anula ao avaliar em zero, de onde $B_{1}=0$.

Por outro lado, $B_{2}$ é uma soma de termos da forma:

$$
\text { const } \partial_{g_{m}}^{\text {(par) }}\left(\prod_{L \in M} g_{L}\right) \partial_{g_{m}}^{\text {(impar) }} \exp \sum_{\substack{\Gamma \in \mathcal{G}^{\prime}(\Lambda) \\ \Gamma \nsim M}} c_{\Gamma} \mu^{\Gamma},
$$

que também são nulos pelo mesmo argumento pelo qual é nulo o $A_{2}$ na parte (a).

\subsubsection{Lema:}

(a)

$$
\left.\partial_{g_{m}} \mathcal{S}_{n}\left(i_{1}, \ldots, i_{n}\right)\right|_{g_{m}=0}=\left.\sum_{\mathbf{x} \in \mathbb{Z}^{d}} \mathcal{S}_{n+2}\left(i_{1}, \ldots, i_{n},(m, \mathrm{x}),(m+1, \mathrm{x})\right)\right|_{g_{m}=0}
$$




$$
\begin{aligned}
& \left.\partial_{g_{m}}^{2} \mathcal{S}_{n}\left(i_{1}, \ldots, i_{n}\right)\right|_{g_{m}=0} \\
& =\left.\sum_{\mathbf{x}, \mathbf{y} \in \mathbb{Z}^{d}} \mathcal{S}_{n+4}\left(i_{1}, \ldots, i_{n},(m, \mathrm{x}),(m+1, \mathrm{x}),(m, \mathrm{y}),(m+1, \mathrm{y})\right)\right|_{g_{m}=0} \\
& -\left.\left.\left.\mathcal{S}_{n}\left(i_{1}, \ldots, i_{n}\right)\right|_{g_{m}=0} \sum_{\mathbf{x}, \mathbf{y} \in \mathbb{Z}^{d}} \mathcal{S}_{2}((m, \mathbf{x}),(m, \mathrm{y}))\right|_{g_{m}=0} \mathcal{S}_{2}((m+1, \mathrm{x}),(m+1, \mathrm{y}))\right|_{g_{m}=0}
\end{aligned}
$$

Demonstração: Basta combinar 4.2.4 com 4.3.2(b,a). 


\section{Capítulo 5}

\section{A Função de 2 pontos}

\subsection{Decaimento Exponencial}

Nesta seção particularizaremos alguns dos resultados gerais da seção anterior para o caso da função de 2 pontos.

5.1.1 Lema: Sejam $i, j \in \mathbb{Z}^{d+1}$ e seja $n \in \mathbb{N} \cup\{0\}$. Se $i_{0}<j_{0}$, então:

(a)

$$
\left.\partial_{g_{m}}^{2 n} \mathcal{S}_{2}(i, j)\right|_{g_{m}=0}=0, \quad \text { se } i_{0} \leqslant m<j_{0}
$$

(b)

$$
\left.\partial_{g_{m}}^{2 n+1} \mathcal{S}_{2}(i, j)\right|_{g_{m}=0}=0, \quad \text { se } m<i_{0} \text { ou se } j_{0} \leqslant m .
$$

Se $i_{0}=j_{0}$, então:

(c)

$$
\left.\partial_{g_{m}}^{2 n+1} \mathcal{S}_{2}(i, j)\right|_{g_{m}=0}=0, \quad \forall m
$$

Demonstração: A parte (a) segue de 4.3.2(b). As partes (b) e (c) seguem de 4.3.2(c).

\subsubsection{Lema:}

(a) Se $i_{0} \leqslant m<j_{0}$, então:

$$
\left.\partial_{g_{m}} \mathcal{S}_{2}(i, j)\right|_{g_{m}=0}=\left.\left.\sum_{\mathbf{x} \in \mathbb{Z}^{d}} \mathcal{S}_{2}(i,(m, \mathrm{x}))\right|_{g_{m}=0} \mathcal{S}_{2}((m+1, \mathrm{x}), j)\right|_{g_{m}=0} .
$$


(b) Se $m<i_{0}$, então:

$$
\begin{aligned}
& \left.\partial_{g_{m}}^{2} \mathcal{S}_{2}(i, j)\right|_{g_{m}=0} \\
& =\left.\left.\sum_{\mathrm{x}, \mathrm{y} \in \mathbb{Z}^{d}} \mathcal{S}_{2}((m, \mathrm{x}),(m, \mathrm{y}))\right|_{g_{m}=0} \mathcal{S}_{4}((m+1, \mathrm{x}),(m+1, \mathrm{y}), i, j)\right|_{g_{m}=0} \\
& -\left.\left.\left.\mathcal{S}_{2}(i, j)\right|_{g_{m}=0} \sum_{\mathbf{x}, \mathbf{y} \in \mathbb{Z}^{d}} \mathcal{S}_{2}((m, \mathrm{x}),(m, \mathrm{y}))\right|_{g_{m}=0} \mathcal{S}_{2}((m+1, \mathrm{x}),(m+1, \mathrm{y}))\right|_{g_{m}=0} .
\end{aligned}
$$

(c) Se $j_{0} \leqslant m$, então:

$$
\begin{aligned}
& \left.\partial_{g_{m}}^{2} \mathcal{S}_{2}(i, j)\right|_{g_{m}=0} \\
& =\left.\left.\sum_{\mathbf{x}, \mathbf{y} \in \mathbb{Z}^{d}} \mathcal{S}_{4}(i, j,(m, \mathbf{x}),(m, \mathbf{y}))\right|_{g_{m}=0} \mathcal{S}_{2}((m+1, \mathbf{x}),(m+1, \mathrm{y}))\right|_{g_{m}=0} \\
& -\left.\left.\left.\mathcal{S}_{2}(i, j)\right|_{g_{m}=0} \sum_{\mathbf{x}, \mathbf{y} \in \mathbb{Z}^{d}} \mathcal{S}_{2}((m, \mathbf{x}),(m, \mathbf{y}))\right|_{g_{m}=0} \mathcal{S}_{2}((m+1, \mathbf{x}),(m+1, \mathbf{y}))\right|_{g_{m}=0}
\end{aligned}
$$

Demonstração: A parte (a) segue de 4.3.3(a) e 4.3.2(a). As partes (b) e (c) seguem de $4.3 .3(\mathrm{~b})$ e $4.3 .2(\mathrm{a})$.

5.1.3 Teorema: No modelo de Ising na região de altas temperaturas a função de 2 pontos decai exponencialmente com a separação dos seus argumentos. Ou seja, se $\beta$ é suficientemente pequeno existem $m=m(\beta)$ e $c=c(\beta)$ tais que:

$$
\left|\mathcal{S}_{2}(i, j)\right| \leqslant c \mathrm{e}^{-m\left|i_{0}-j_{0}\right|} .
$$

Demonstração: Por 5.1.1(a) com $n=0$ temos que:

$$
\mathcal{S}_{2}^{\Lambda}(i, j)=g_{i_{0}} g_{i_{0}+1} \cdots g_{j_{0}-1} F\left(g, g_{m}\right),
$$

onde $F$ é uma função analítica. Em particular, no modelo de Ising:

$$
\mathcal{S}_{2}^{\Lambda}(i, j)=g^{\left|i_{0}-j_{0}\right|} F(g)=\mathrm{e}^{-m\left|i_{0}-j_{0}\right|} F(g),
$$

onde $m:=-\ln g>0$ se $0<g<1$. Portanto:

$$
\left|\mathcal{S}_{2}^{\Lambda}(i, j)\right| \leqslant c \mathrm{e}^{-m\left|i_{0}-j_{0}\right|},
$$

de onde segue o lema tomando limite em $\Lambda$. 


\subsubsection{Corolários:}

(a) Unicidade do vácuo: $\Omega$ é o único vetor (salvo múltiplos escalares de sí própio) em $\mathcal{H}$ tal que $H \Omega=0$.

(b) Gap de massa inferior: $\sigma(H) \subseteq\{0\} \cup[m, \infty)$.

(c) $\widehat{S}_{2}\left(p_{0}, \mathbf{p}\right)$ é função analítica de $p_{0}$ na região $\left|\operatorname{Im} p_{0}\right|<m$.

(d)

$$
\widehat{S}_{2}\left(p_{0}, \mathrm{p}\right)=\int_{[0, \infty)} \operatorname{Re}\left(\frac{1+\mathrm{e}^{-\left(\lambda_{0}+i p_{0}\right)}}{1-\mathrm{e}^{-\left(\lambda_{0}+i p_{0}\right)}}\right) d \rho\left(\lambda_{0}, \mathrm{p}\right),
$$

onde, para cada $\mathrm{p} \in T^{d}, d \rho\left(\lambda_{0}, \mathrm{p}\right)$ é uma medida suportada em $\lambda_{0} \in\{0\} \cup$ $[m, \infty)$.

Referências: Para as parte (a) e (b) veja-se [93] Theorem 6, pp. 134-135. ${ }^{1}$ Basicamente, observe que:

$$
\begin{aligned}
\left|\left\langle P_{0} \Omega, \mathrm{e}^{-\left|x_{0}\right| H} P_{0} \Omega\right\rangle-\left\langle P_{0} \Omega, \Omega\right\rangle\left\langle\Omega, P_{0} \Omega\right\rangle\right| & =\left|S_{2}\left(x_{0}, 0\right)-\mu\left(p_{0}\right) \mu\left(p_{0}\right)\right| \\
& =\left|\mu\left(p_{0} p_{\left(x_{0}, 0\right)}\right)-\mu\left(p_{0}\right) \mu\left(p_{0}\right)\right| \leqslant c \mathrm{e}^{-m\left|x_{0}\right|},
\end{aligned}
$$

e portanto:

$$
\begin{array}{r}
\inf \left(\operatorname{supp} d\left\langle\varphi, E_{\lambda} \varphi\right\rangle\right)=-\lim _{x_{0} \rightarrow \infty} \frac{1}{\left|x_{0}\right|} \ln \left(\left\langle P_{0} \Omega, \mathrm{e}^{-\left|x_{0}\right| H} P_{0} \Omega\right\rangle-\left\langle P_{0} \Omega, \Omega\right\rangle\left\langle\Omega, P_{0} \Omega\right\rangle\right) \\
\geqslant-\lim _{x_{0} \rightarrow \infty} \frac{1}{\left|x_{0}\right|}\left(\ln c-m\left|x_{0}\right|\right)=m
\end{array}
$$

onde $\varphi:=P_{0} \Omega-\left\langle\Omega, P_{0} \Omega\right\rangle \Omega$.

A parte (c) é conseqüência de A.1 no apêndice no final desta seção. Para a parte (d), observe que transformando Fourier e trocando formalmente a ordem de integração na última identidade da seção 2.1 temos:

$$
\begin{array}{r}
\widehat{S}_{2}\left(p_{0}, \mathrm{p}\right) \\
=\frac{1}{(2 \pi)^{\frac{d+1}{2}}} \int_{[0, \infty)} \int_{T^{d}}\left(\sum_{x_{0} \in \mathbb{Z}} \mathrm{e}^{-\left|x_{0}\right| \lambda_{0}} \mathrm{e}^{-i p_{0} x_{0}}\right)\left(\sum_{\mathbf{x} \in \mathbb{Z}^{d}} \mathrm{e}^{i \mathbf{x} \cdot \lambda} \mathrm{e}^{-i \mathbf{x} \cdot \mathbf{p}}\right) d \mu\left(\lambda_{0}, \lambda\right) \\
=\int_{[0, \infty)} \operatorname{Re}\left(\frac{1+\mathrm{e}^{-\left(\lambda_{0}+i p_{0}\right)}}{1-\mathrm{e}^{-\left(\lambda_{0}+i p_{0}\right)}}\right) d \rho\left(\lambda_{0}, \mathbf{p}\right),
\end{array}
$$

\footnotetext{
${ }^{1}$ Cf. [37] pp. 162-163, [59] pp. 314-315.
} 
onde temos denotado $d \rho\left(\lambda_{0}, \mathrm{p}\right):=(2 \pi)^{-\frac{d-1}{2}} \int_{T^{d}} \delta(\boldsymbol{\lambda}-\mathrm{p}) d \mu\left(\lambda_{0}, \boldsymbol{\lambda}\right)$. Esta identidade vale rigorosamente no sentido das distribuições e está demonstrada em [84] pp. 218219. via: $^{2}$

No restante desta seção vamos considerar $\mathcal{S}_{2}$ como operador linear em $\ell^{2}\left(\mathbb{Z}^{d+1}\right)$

$$
\mathcal{S}_{2}\left(\left\{a_{j}\right\}_{j \in \mathbb{Z}^{d+1}}\right)_{i}:=\sum_{j \in \mathbb{Z}^{d+1}} \mathcal{S}_{2}(i, j) a_{j} .
$$

Dado $m \in \mathbb{Z}$ sejam:

$$
\begin{aligned}
& \ell_{\leqslant m}^{2}\left(\mathbb{Z}^{d+1}\right):=\left\{\left\{a_{j}\right\}_{j \in \mathbb{Z}^{d+1}} \in \ell^{2}\left(\mathbb{Z}^{d+1}\right): a_{j}=0 \text { se } j_{0}>m\right\} \\
& \ell_{>m}^{2}\left(\mathbb{Z}^{d+1}\right):=\left\{\left\{a_{j}\right\}_{j \in \mathbb{Z}^{d+1}} \in \ell^{2}\left(\mathbb{Z}^{d+1}\right): a_{j}=0 \text { se } j_{0} \leqslant m\right\} .
\end{aligned}
$$

Observe que $\ell^{2}\left(\mathbb{Z}^{d+1}\right)=\ell_{\leqslant m}^{2}\left(\mathbb{Z}^{d+1}\right) \oplus \ell_{>m}^{2}\left(\mathbb{Z}^{d+1}\right)$.

\subsubsection{Lema:}

(a) $\ell_{\leqslant m}^{2}\left(\mathbb{Z}^{d+1}\right)$ e $\ell_{>m}^{2}\left(\mathbb{Z}^{d+1}\right)$ são invariantes por $\left.\mathcal{S}_{2}\right|_{g_{m}=0}$.

(b) $\left.\mathcal{S}_{2}\right|_{g_{m}=0}$ é soma direta de dois operadores agindo independentemente em cada um destes subespaços.

(c) Existe $\mathcal{S}_{2}^{-1}=: \Gamma$.

(d) $\left.\Gamma\right|_{g_{m}=0}$ é soma direta de dois operadores agindo independentemente em cada um dos subespaços do item (a).

Demonstração: (a) é conseqüência direta das definições e 5.1.1(a) com $n=0$; (b) é corolário de (a); (c) segue basicamente de 5.1.3, veja-se [69] Lemma A2 pp. 605-606 para uma prova. Finalmente (d) também segue de (b).

5.1.6 Observação: $\Gamma$ é simétrico, i.e., $\Gamma(i, j)=\Gamma(j, i)$.

\section{Apêndice à Seção 5.1: Decaimento Exponencial e Analiticidade}

A.1 Lema: Seja $g \in L^{1}(\mathbb{R})$. Então, $|g(x)| \leqslant e^{-m|x|}$ implica que $\widehat{g}(p)$ é analítica na faixa $|\operatorname{Im} p|<m$.

\footnotetext{
${ }^{2}$ Isto está bem definido; veja-se [69] p. 606.
} 
Demonstração: ${ }^{3}$ Seja $\gamma$ uma curva simples fechada na faixa $|\operatorname{Im} p|<m$. Observe que:

$$
\oint_{\gamma} d p \int_{\mathbb{R}} d x\left|\mathrm{e}^{-i p x} g(x)\right| \leqslant \oint_{\gamma} d p \int_{\mathbb{R}} d x \mathrm{e}^{(\operatorname{Im} p) x-m|x|}<\infty,
$$

de onde, pelo Teorema de Fubini:

$$
\oint_{\gamma} d p \widehat{g}(p)=\oint_{\gamma} d p \int_{\mathbb{R}} d x \mathrm{e}^{-i p x} g(x)=\int_{\mathbb{R}} d x g(x) \oint_{\gamma} d p \mathrm{e}^{-i p x}=0
$$

de onde segue o lema, pelo Teorema de Morera. ${ }^{4}$

\subsection{O Inverso da Função de 2 pontos}

Nesta seção serão estudadas propriedades de decaimento exponencial para $\Gamma$ análogas às de $\mathcal{S}_{2}$.

5.2.1 Lema: Sejam $i, j \in \mathbb{Z}^{d+1}$. Se $i_{0}<j_{0}$, então:

(a)

$$
\left.\Gamma(i, j)\right|_{g_{m}=0}=0 \text { se } i_{0} \leqslant m<j_{0} .
$$

(b)

$$
\left.\partial_{g_{m}} \Gamma(i, j)\right|_{g_{m}=0}= \begin{cases}0 & \text { se } j_{0} \leqslant m \\ 0 & \text { se } i_{0} \leqslant m<j_{0} \text { quando }\left|i_{0}-j_{0}\right| \geqslant 2 \\ 0 & \text { se } m<i_{0}\end{cases}
$$

Se $i_{0}=j_{0}$, então:

(c)

$$
\left.\partial_{g_{m}} \Gamma(i, j)\right|_{g_{m}=0}=0 \forall m .
$$

${ }^{3}$ Cf. [19], Appendix A, p. 192.

${ }^{4}$ Veja-se, $v . g .,[22]$ p. 88. 
Demonstração: (a) segue de 5.1.5(d) usando reductio ad absurdum. Para o item (b) no caso $i_{0} \leqslant m<j_{0}$, usando 5.2.2 infra $\operatorname{com} n=1$ e 5.1.2(a) temos:

$$
\begin{aligned}
\left.\partial_{g_{m}} \Gamma(i, j)\right|_{g_{m}=0}=-\left.\left.\left.\sum_{a, b \in \mathbb{Z}^{d+1}} \Gamma(i, a)\right|_{g_{m}=0} \partial_{g_{m}} \mathcal{S}_{2}(a, b)\right|_{g_{m}=0} \Gamma(b, j)\right|_{g_{m}=0} \\
=-\left.\left.\left.\left.\sum_{a, b \in \mathbb{Z}^{d+1}} \sum_{\mathbf{x} \in \mathbb{Z}^{d}} \Gamma(i, a)\right|_{g_{m}=0} \mathcal{S}_{2}(a,(m, \mathrm{x}))\right|_{g_{m}=0} \mathcal{S}_{2}((m+1, \mathrm{x}), b)\right|_{g_{m}=0} \Gamma(b, j)\right|_{g_{m}=0} \\
=-\sum_{\mathbf{x} \in \mathbb{Z}^{d}} \delta_{i(m, \mathbf{x})} \delta_{(m+1, \mathbf{x}) j}
\end{aligned}
$$

sendo esta última expressão nula se $\left|i_{0}-j_{0}\right| \geqslant 2$. Os outros casos e o ponto (c) se provam usando 5.2.2 analogamente, acrescentando o item (a) anterior junto con $5.1 .1(b, c)$.

O lema 5.2.1 anterior pode generalizar-se para derivadas superiores. Mas antes é preciso introduzir o seguinte resultado auxiliar que se prova facilmente por indução:

5.2.2 Lema: Para cada $n \in \mathbb{N}$ tem-se:

$$
\partial_{g_{m}}^{n} \Gamma=-\Gamma\left(\partial_{g_{m}}^{n}\left(\mathcal{S}_{2} \Gamma\right)-\mathcal{S}_{2} \partial_{g_{m}}^{n} \Gamma\right)
$$

Ainda que formalmente a igualdade acima seja trivial (o primeiro sumando dentro do parêntese é nulo e a fórmula se reduz a uma identidade), a mesma reveste-se de certa importância computacional no cálculo iterativo da derivada $n$-ésima de $\Gamma$, pois no membro direito aparecem derivadas de $\Gamma$ de ordem até $n-1$.

5.2.3 Lema: Sejam $i, j \in \mathbb{Z}^{d+1}$. Se $n \in \mathbb{N} \cup\{0\}$, então:

$n$ par $\left.\Longrightarrow \partial_{g_{m}}^{n} \Gamma(i, j)\right|_{g_{m}=0}=0 \forall i_{0} \leqslant m<j_{0}$ se $i_{0}<j_{0}$

$$
\begin{array}{r}
n \text { ímpar }\left.\Longrightarrow \partial_{g_{m}}^{n} \Gamma(i, j)\right|_{g_{m}=0}=0 \text { se } m<i_{0} \text { ou } j_{0} \leqslant m \text { no caso } i_{0}<j_{0} \\
\text { ou } \forall m \text { no caso } i_{0}=j_{0} .
\end{array}
$$

Demonstração: Por indução em $n$. O caso $n=0$ é 5.2.1(a) e o caso $n=1$ é 5.2 .1 (b,c). Suponhamos que o enunciado do lema é válido para cada $i: i \leqslant n-1$. De 5.2.2 
temos:

$$
\left.\partial_{g_{m}}^{n} \Gamma(i, j)\right|_{g_{m}=0}=-\left.\left.\left.\sum_{a, b \in \mathbb{Z}^{d+1}} \sum_{k=0}^{n-1}\left(\begin{array}{l}
n \\
k
\end{array}\right) \Gamma(i, a)\right|_{g_{m}=0} \partial_{g_{m}}^{n-k} \mathcal{S}_{2}(a, b)\right|_{g_{m}=0} \partial_{g_{m}}^{k} \Gamma(b, j)\right|_{g_{m}=0}
$$

- Se $n$ é par com $i_{0}<j_{0}$ e $i_{0} \leqslant m<j_{0}$, então 5.2.1(a) implica que o primeiro fator na somatória do membro direito acima é nulo a menos que $a$ esteja em ou abaixo de $L_{m}$.

- Agora, quando o índice $k$ na somatória acima é par, a hipótese indutiva implica que o terceiro fator de cada somando é nulo a menos que $b$ esteja acima de $L_{m}$. Mas neste caso $n-k$ também resulta par e assim, por 5.1.2(a), o segundo fator é nulo se $a_{0} \leqslant m<b_{0}$.

- Por outro lado, quando o índice $k$ é ímpar, a hipótese indutiva implica que o terceiro fator é nulo a menos que $b$ esteja em ou abaixo de $L_{m}$. Mas neste caso $n-k$ resulta ímpar e assim, por 5.1.2(b, c), o segundo fator é nulo se $a_{0}, b_{0} \leqslant m$.

- Se $n$ é ímpar com $i_{0}<j_{0}$ e $m<i_{0}$ (respectivamente $j_{0} \leqslant m$ ), então 5.2.1(a) implica que o primeiro fator na somatória acima é nulo a menos que $a$ esteja acima (resp. em ou abaixo) de $L_{m}$.

- Quando o índice $k$ é par, a hipótese indutiva implica que o terceiro fator é nulo a menos que $b$ esteja acima (resp. em ou abaixo) de $L_{m}$. Mas neste caso $n-k$ resulta ímpar e assim, por $5.1 .2(\mathrm{~b}, \mathrm{c})$, o segundo fator é nulo se $m<a_{0}, b_{0}$ (resp. $\left.a_{0}, b_{0} \leqslant m\right)$.

- Quando o índice $k$ é ímpar, a hipótese indutiva implica que o terceiro fator é nulo a menos que $b$ esteja em ou abaixo (resp. acima) de $L_{m}$. Mas neste caso $n-k$ resulta par e assim, por 5.1.2(a), o segundo fator é nulo se $b_{0} \leqslant m<a_{0}$ (resp. $a_{0} \leqslant m<b_{0}$ ).

No caso $n$ ímpar com $i_{0}=j_{0}$ a prova é análoga, distinguindo dois subcasos:

$$
\begin{aligned}
& -m<i_{0}=j_{0} \\
& -i_{0}=j_{0} \leqslant m
\end{aligned}
$$

Finalmente, 
5.2.4 Teorema: No modelo de Ising na região de altas temperaturas, se $\left|i_{0}-j_{0}\right| \geqslant$ 2, então:

$$
|\Gamma(i, j)| \leqslant \text { const } \mathrm{e}^{-3 m\left|i_{0}-j_{0}\right|}
$$

Demonstração: É análoga à de 5.1.3, usando 5.2.1(a, b) e 5.2.3 com $n=2$. Com relação a este resultado, Cf. o comentário (1) que segue ao lema 2.2 em [69] p. 600.

\subsubsection{Corolários:}

(a) $\widehat{\Gamma}\left(p_{0}, \mathrm{p}\right)$ é função analítica de $p_{0}$ na região $\left|\operatorname{Im} p_{0}\right|<3 m$.

(b)

$$
\widehat{S}_{2}\left(p_{0}, \mathbf{p}\right)=Z(\mathbf{p}) \operatorname{Re}\left(\frac{1+\mathrm{e}^{-\left(\omega(\mathrm{p})+i p_{0}\right)}}{1-\mathrm{e}^{-\left(\omega(\mathbf{p})+i p_{0}\right)}}\right)+\int_{3 m}^{\infty} \operatorname{Re}\left(\frac{1+\mathrm{e}^{-\left(\lambda_{0}+i p_{0}\right)}}{1-\mathrm{e}^{-\left(\lambda_{0}+i p_{0}\right)}}\right) d \rho\left(\lambda_{0}, \mathbf{p}\right),
$$

onde $Z(\mathrm{p})$ é uma função $C^{\infty}$ positiva e $\omega(\mathrm{p})$ é uma função real analítica com $m \leqslant \omega(\mathrm{p})<3 m$.

Referências: A parte (a) é conseqüência de A.1 do apêndice no final da seção 5.1. Usando (a), a prova da parte (b) é como a prova do teorema 2.5 em [84]. 


\section{Capítulo 6}

\section{As Funções de 4 pontos}

\subsection{A Parte Não-Conexa}

Começamos estabelecendo notação e definições que serão usadas não somente na presente seção, senão ao longo de todo este capítulo.

Dados 4 sítios $i, j, k, l \in \mathbb{Z}^{d+1}$ com $i_{0} \leqslant j_{0} \leqslant k_{0} \leqslant l_{0}$ a 4 -upla $\left(i_{0}, j_{0}, k_{0}, l_{0}\right) \in$ $\mathbb{Z}^{4}$ será dita a configuração por eles determinada. A configuração é dita nãodegenerada no caso $i_{0}<j_{0}<k_{0}<l_{0}$ e degenerada caso contrário. No seguinte, salvo menção explícita em contrário, configuração significará configuração não degenerada.

Lembremos que na seção 3.2 p. 34 a parte não-conexa da função de 4 pontos foi definida como:

$$
\mathcal{D}_{0}(i, j, k, l)=\mathcal{S}_{2}(i, k) \mathcal{S}_{2}(j, l)+\mathcal{S}_{2}(i, l) \mathcal{S}_{2}(j, k)
$$

6.1.1 Observação: Resulta simples verificar que $\mathcal{D}_{0}$ é invariante pelas operações:

$A_{1}:=$ permutação de $i$ por $j$

$A_{2}:=$ permutação de $k$ por $l$

$B:=$ permutação simultânea de $i$ por $k$ e $j$ por $l$.

Esta observação motiva a seguinte definição. Diremos que duas configurações são equivalentes ou do mesmo tipo se uma pode ser obtida da outra pela aplicação de alguma das operações $A_{1}, A_{2}, A_{1} A_{2}, B, A_{1} B, A_{2} B$ e $A_{1} A_{2} B$. Assim, para cada configuração existem outras sete que lhe são equivalentes, e portanto o conjunto 
das $4 !=24$ diferentes configurações determinadas por 4 sítios fica dividido em três classes de equivalência ou tipos. De cada uma destas classes escolhemos os seguintes representantes:

$$
\left(i_{0}, j_{0}, k_{0}, l_{0}\right),\left(i_{0}, k_{0}, j_{0}, l_{0}\right) \text { e }\left(i_{0}, l_{0}, k_{0}, j_{0}\right),
$$

que serão chamadas configuração 1,2 e 3 respectivamente. Nenhuma delas pode ser obtida duma outra pela aplicação reiterada das operações $A_{1}, A_{2}, B$.

A equivalência de configurações reflete as propriedades de simetria de $\mathcal{D}_{0}$ no seguinte sentido: supondo conhecido o valor $F(i, j, k, l)$ de $\mathcal{D}_{0}(i, j, k, l)$ quando os sítios $i, j, k, l$ determinam uma dada configuração, o valor de $\mathcal{D}_{0}(i, j, k, l)$ quando os sítios determinam uma outra configuração obtida da original pela aplicação de alguma das operações de simetria $A$ em 6.1.1, está dado por $F(A(i, j, k, l))$. Observe que idênticas considerações seguem para as derivadas com respeito a $g_{m}$ (pois é uma variável independente dos sítios), os inversos (pela unicidade do inverso) e as derivadas dos inversos (pelos dois motivos anteriores) de $\mathcal{D}_{0}$.

Em $\ell^{2}\left(\mathbb{Z}^{d+1} \times \mathbb{Z}^{d+1}\right)$ definimos os subespaços simétrico e anti-simétrico respectivamente por:

$$
\begin{aligned}
& \ell_{s}^{2}\left(\mathbb{Z}^{d+1} \times \mathbb{Z}^{d+1}\right):=\left\{\left\{a_{i j}\right\}_{i, j \in \mathbb{Z}^{d+1}} \in \ell^{2}\left(\mathbb{Z}^{d+1} \times \mathbb{Z}^{d+1}\right): a_{i j}=a_{j i}\right\} \\
& \ell_{a}^{2}\left(\mathbb{Z}^{d+1} \times \mathbb{Z}^{d+1}\right):=\left\{\left\{a_{i j}\right\}_{i, j \in \mathbb{Z}^{d+1}} \in \ell^{2}\left(\mathbb{Z}^{d+1} \times \mathbb{Z}^{d+1}\right): a_{i j}=-a_{j i}\right\}
\end{aligned}
$$

É simples verificar as seguintes

\subsubsection{Observações:}

(a) Como operador em $\ell^{2}\left(\mathbb{Z}^{d+1} \times \mathbb{Z}^{d+1}\right)$, $\mathcal{D}_{0}$ é nulo no espaço anti-simétrico.

(b) Como operador no espaço simétrico, tem-se:

$$
\mathcal{D}_{0}^{-1}(i, j, k, l)=\frac{1}{2} \Gamma(i, k) \Gamma(j, l) .
$$

(c) $\mathcal{D}_{0}^{-1}$ tem as mesmas simetrias que $\mathcal{D}_{0}$ mais a simetria adicional:

$$
A_{3}:=\text { permutação de } j \text { por } l \text {. }
$$

Para facilitar o enunciado e as provas de certos resultados, introduzimos as seguintes definições. Dada uma configuração $\left(i_{0}, j_{0}, k_{0}, l_{0}\right)$, os conjuntos:

$$
\left\{m \in \mathbb{Z}: k_{0} \leqslant m<l_{0}\right\}
$$




$$
\begin{aligned}
& \left\{m \in \mathbb{Z}: j_{0} \leqslant m<k_{0}\right\} \\
& \left\{m \in \mathbb{Z}: i_{0} \leqslant m<j_{0}\right\}
\end{aligned}
$$

serão chamados de as partes de cima, do meio e de baixo da configuração, respectivamente. $\mathrm{O}$ complemento da união das três partes será chamada de parte de fora, no entanto a união das partes de cima com a de baixo será dita as bordas da configuração.

Uma configuração será dita do tipo A se os sítios $i$ e $l$ estam ambos ou bem na parte de cima ou bem na de baixo. Igualmente, uma configuração é do tipo A se é equivalente a uma do tipo 1 pela permutação de $j$ por $l$ (observe-se que as configurações do tipo 1 dejam os sítios $i$ e $j$ ambos na parte de cima ou na parte de baixo). Análogamente, uma configuração será dita do tipo $\mathbf{B}$ se os sítios $i$ e $k$ estam ambos ou bem na parte de cima ou bem na de baixo. As configurações de tipo A e B existem apenas entre as de tipo 2 e 3 . As configurações de tipo 1 , A e B são relevantes para determinado tipo de resultado, pois qualquer enunciado relativo a uma delas na parte do meio é automáticamente válido para todas as do seu mesmo tipo ("preservam a parte do meio"). Por 6.1.2(c), para o estudo de $\mathcal{D}_{0}^{-1}$, as configurações 1 e A são equivalentes (na verdade, $\mathcal{D}_{0}^{-1}$ também admite a simetria de permutação de $i$ por $k$, mas a conseqüência disso também é a equivalência das configurações 1 e A).

\subsubsection{Lema: Para qualquer configuração tem-se:}

(a) $\left.\partial_{g_{m}}^{2 n} \mathcal{D}_{0}^{-1}(i, j, k, l)\right|_{g_{m}=0}=0$ nas bordas. Além disso, nos casos $n=0,1$ a derivada correspondente é nula também no meio da configuração 1.

(b) $\left.\partial_{g_{m}}^{2 n-1} \mathcal{D}_{0}^{-1}(i, j, k, l)\right|_{g_{m}=0}=0$ no meio. Além disso, no caso $n=1$ a derivada também é nula nas bordas de todas as configurações.

Demonstração: Para a parte (a), observe que, usando 6.1.2(b), temos:

$$
\left.\partial_{g_{m}}^{2 n} \mathcal{D}_{0}^{-1}(i, j, k, l)\right|_{g_{m}=0}=A_{1}+A_{2}
$$

onde $A_{1}$ é uma soma de termos da forma:

$$
\text { const }\left.\left.\quad \partial_{g_{m}}^{(\text {par })} \Gamma(i, k)\right|_{g_{m}=0} \partial_{g_{m}}^{(\text {par })} \Gamma(j, l)\right|_{g_{m}=0}
$$

e, usando 5.2.3, tal termo vale: 


\begin{tabular}{r||c|c} 
& 1 e $\mathrm{A}$ & $\mathrm{B}$ \\
\hline \hline acima & 0 & 0 \\
\hline meio & 0 & $A_{1}$ \\
\hline abaixo & 0 & 0
\end{tabular}

(aqui, na primeira linha é indicado o tipo da configuração). Analogamente, $A_{2}$ é uma soma de termos da forma:

$$
\text { const }\left.\left.\quad \partial_{g_{m}}^{(\text {impar })} \Gamma(i, k)\right|_{g_{m}=0} \partial_{g_{m}}^{(\text {impar })} \Gamma(j, l)\right|_{g_{m}=0}
$$

e, usando 5.2.3, tal termo vale:

\begin{tabular}{r||c|c} 
& 1 e $\mathrm{A}$ & $\mathrm{B}$ \\
\hline \hline acima & 0 & 0 \\
\hline meio & $A_{2}$ & 0 \\
\hline abaixo & 0 & 0
\end{tabular}

Observe que $A_{2}=0$ no caso $n=0$ (ou seja, sem derivar) e que no caso $n=1$ (ou seja, a derivada de segunda ordem) as derivadas ímpares de ordem mais alta que aparecem em $A_{2}$ são as de primeira ordem, que são nulas por 5.2.1(b).

Para a parte (b), temos analogamente:

$$
\left.\partial_{g_{m}}^{2 n-1} \mathcal{D}_{0}^{-1}(i, j, k, l)\right|_{g_{m}=0}=B_{1}+B_{2}
$$

onde $B_{1}$ é uma soma de termos da forma:

$$
\text { const }\left.\left.\quad \partial_{g_{m}}^{(\text {impar })} \Gamma(i, k)\right|_{g_{m}=0} \partial_{g_{m}}^{(\text {par })} \Gamma(j, l)\right|_{g_{m}=0}
$$

e, por 5.2.3, tal termo é nulo no meio. Analogamente, $B_{2}$ é uma soma de termos da forma:

$$
\text { const }\left.\left.\partial_{g_{m}}^{(\text {par })} \Gamma(i, k)\right|_{g_{m}=0} \partial_{g_{m}}^{(\text {impar) }} \Gamma(j, l)\right|_{g_{m}=0}
$$

e, por 5.2.3, tal termo também é nulo no meio. Observe que no caso $n=1$ (ou seja, a derivada de primeira ordem) as derivadas ímpares de ordem mais alta que aparecem em $B_{1}$ e $B_{2}$ são as de primeira ordem, que são nulas por 5.2.1(b).

\subsection{A Função de 4 pontos}

Apresentamos aqui resultados auxiliares que serão usados nas provas da próxima seção. Estes resultados são basicamente casos particulares dos resultados gerais 
consignados no final da seção 4.3, p. 84 et ss..

Para abreviar, no seguinte denotaremos:

$$
\delta(m):=\left.\left.\sum_{\mathbf{x}, \mathbf{y} \in \mathbb{Z}^{d}} \mathcal{S}_{2}((m, \mathrm{x}),(m, \mathrm{y}))\right|_{g_{m}=0} \mathcal{S}_{2}((m+1, \mathrm{x}),(m+1, \mathrm{y}))\right|_{g_{m}=0} .
$$

Em alguns resultados aparecerão sítios sub-indexados $i_{k_{m}}$. Em todos os casos supõese que $i_{k_{m}} \in\{i, j, k, l\}$. Estabelecemos também a seguinte convenção: o acento circunflexo num dado síto $\widehat{i_{k_{m}}}$ significa que tal síto deve omitir-se.

\subsubsection{Lema:}

(a) $\left.\mathcal{S}_{4}(i, j, k, l)\right|_{g_{m}=0}$ toma os seguintes valores:

\begin{tabular}{|c||c|c|c|}
\hline & 1 & $\mathrm{~A}$ & $\mathrm{~B}$ \\
\hline \hline ac. & 0 & 0 & 0 \\
\hline $\mathrm{m}$. & $\left.\left.\mathcal{S}_{2}(i, j)\right|_{g_{m}=0} \mathcal{S}_{2}(k, l)\right|_{g_{m}=0}$ & $\left.\left.\mathcal{S}_{2}(i, l)\right|_{g_{m}=0} \mathcal{S}_{2}(k, j)\right|_{g_{m}=0}$ & $\left.\left.\mathcal{S}_{2}(i, k)\right|_{g_{m}=0} \mathcal{S}_{2}(j, l)\right|_{g_{m}=0}$ \\
\hline ab. & 0 & 0 & 0 \\
\hline
\end{tabular}

(b) $\left.\partial_{g_{m}} \mathcal{S}_{4}(i, j, k, l)\right|_{g_{m}=0}$ toma os seguintes valores:

$$
\left.\left.\sum_{\mathbf{x} \in \mathbb{Z}^{d}} \mathcal{S}_{4}\left(i_{k_{1}}, \ldots, \widehat{i_{k_{r}}}, \ldots, i_{k_{4}},(m, \mathbf{x})\right)\right|_{g_{m}=0} \mathcal{S}_{2}\left((m+1, \mathbf{x}), i_{k_{r}}\right)\right|_{g_{m}=0}
$$

na parte de cima de qualquer configuração com $\left(i_{k_{r}}\right)_{0}=\max \left\{i_{0}, j_{0}, k_{0}, l_{0}\right\}$, e

$$
\left.\left.\sum_{\mathbf{x} \in \mathbb{Z}^{d}} \mathcal{S}_{2}\left(i_{k_{r}},(m, \mathrm{x})\right)\right|_{g_{m}=0} \mathcal{S}_{4}\left((m+1, \mathrm{x}), i_{k_{1}}, \ldots, \widehat{i_{k_{r}}}, \ldots, i_{k_{4}}\right)\right|_{g_{m}=0}
$$

na parte de baixo de qualquer configuração $\operatorname{com}\left(i_{k_{r}}\right)_{0}=\min \left\{i_{0}, j_{0}, k_{0}, l_{0}\right\}$.

(c) No meio da configuração 1 tem-se:

$$
\begin{aligned}
& \left.\partial_{g_{m}}^{2} \mathcal{S}_{4}(i, j, k, l)\right|_{g_{m}=0} \\
& \begin{aligned}
=\left.\left.\sum_{\mathrm{x}, \mathrm{y} \in \mathbb{Z}^{d}} \mathcal{S}_{4}(i, j,(m, \mathrm{x}),(m, \mathrm{y}))\right|_{g_{m}=0} \mathcal{S}_{4}((m+1, \mathrm{x}),(m+1, \mathrm{y}), k, l)\right|_{g_{m}=0} \\
\quad-\left.\left.\mathcal{S}_{2}(i, j)\right|_{g_{m}=0} \mathcal{S}_{2}(k, l)\right|_{g_{m}=0} \delta(m) .
\end{aligned}
\end{aligned}
$$

(d) As derivadas de ordem par são nulas nas bordas de qualquer configuração. 
(e) As derivadas de ordem impar são nulas no meio e fora de qualquer configuração.

Demonstração: A parte (a) segue de 4.3.2(a, b). A parte (b) segue de 4.3.3(a) e 4.3.2(a,c). A parte(c) segue de 4.3.3(b) e 4.3.2(a). Finalmente, (d) e (e) seguem de 4.3.2(b, c).

\subsubsection{Lema:}

(a) $\left.\left.\mathcal{S}_{2}(i, j)\right|_{g_{m}=0} \mathcal{S}_{2}(k, l)\right|_{g_{m}=0}$ toma os seguintes valores:

\begin{tabular}{|r||c|c|c|}
\hline & 1 & $\mathrm{~A}$ & $\mathrm{~B}$ \\
\hline \hline acima & 0 & 0 & 0 \\
\hline meio & $\left.\left.\mathcal{S}_{2}(i, j)\right|_{g_{m}=0} \mathcal{S}_{2}(k, l)\right|_{g_{m}=0}$ & 0 & 0 \\
\hline abaixo & 0 & 0 & 0 \\
\hline
\end{tabular}

(b) $\left.\partial_{g_{m}}\left[\mathcal{S}_{2}(i, j) \mathcal{S}_{2}(k, l)\right]\right|_{g_{m}=0}$ toma os seguintes valores:

$$
\left.\left.\mathcal{S}_{2}\left(i_{k_{1}}, i_{k_{2}}\right)\right|_{g_{m}=0} \partial_{g_{m}} \mathcal{S}_{2}\left(i_{k_{3}}, i_{k_{4}}\right)\right|_{g_{m}=0}
$$

na parte de cima de qualquer configuração com $\left\{i_{k_{1}}, i_{k_{2}}\right\}<\left\{i_{k_{3}}, i_{k_{4}}\right\}$, e

$$
\left.\left.\partial_{g_{m}} \mathcal{S}_{2}\left(i_{k_{1}}, i_{k_{2}}\right)\right|_{g_{m}=0} \mathcal{S}_{2}\left(i_{k_{3}}, i_{k_{4}}\right)\right|_{g_{m}=0}
$$

na parte de baixo de qualquer configuração com $\left\{i_{k_{1}}, i_{k_{2}}\right\}<\left\{i_{k_{3}}, i_{k_{4}}\right\}$.

(c) No meio da configuração 1 tem-se:

$$
\begin{aligned}
& \left.\partial_{g_{m}}^{2}\left[\mathcal{S}_{2}(i, j) \mathcal{S}_{2}(k, l)\right]\right|_{g_{m}=0} \\
& =\left.\left[\left.\left.\sum_{\mathbf{x}, \mathbf{y} \in \mathbb{Z}^{d}} \mathcal{S}_{4}(i, j,(m, \mathrm{x}),(m, \mathrm{y}))\right|_{g_{m}=0} \mathcal{S}_{2}((m+1, \mathrm{x}),(m+1, \mathrm{y}))\right|_{g_{m}=0}\right] \mathcal{S}_{2}(k, l)\right|_{g_{m}=0} \\
& -\left.\left.2 \mathcal{S}_{2}(i, j)\right|_{g_{m}=0} \mathcal{S}_{2}(k, l)\right|_{g_{m}=0} \delta(m) \\
& +\left.\left.\left.\mathcal{S}_{2}(i, j)\right|_{g_{m}=0} \sum_{\mathbf{x}, \mathbf{y} \in \mathbb{Z}^{d}} \mathcal{S}_{2}((m, \mathrm{x}),(m, \mathrm{y}))\right|_{g_{m}=0} \mathcal{S}_{4}((m+1, \mathrm{x}),(m+1, \mathrm{y}), k, l)\right|_{g_{m}=0} .
\end{aligned}
$$

(d) As derivadas de ordem par são nulas nas bordas de qualquer configuração. 
(e) As derivadas de ordem impar são nulas no meio e fora de qualquer configuração.

Demonstração: A partes (a) e (b) seguem de 5.1.1(a, b). A parte (c) segue de 5.1.2(b, c), observando que o produto das derivadas (de primeira ordem) é nulo por 5.1.1(b). Finalmente, (d) e (e) seguem de 5.1.1.

\subsection{A Parte Conexa}

Na seção 3.2 pp. 33-34 foi definida a parte conexa da função de 4 pontos por:

$$
\mathcal{D}(i, j, k, l)=\mathcal{S}_{4}(i, j, k, l)-\mathcal{S}_{2}(i, j) \mathcal{S}_{2}(k, l) .
$$

É simples verificar que $\mathcal{D}$ satisfaz as mesmas propriedades de simetria que $\mathcal{D}_{0}$ estabelecidas em 6.1 .1 e também a propriedade 6.1.2(a).

\subsubsection{Lema:}

(a) $\left.\mathcal{D}(i, j, k, l)\right|_{g_{m}=0}$ toma os seguintes valores:

\begin{tabular}{|r||c|c|c|}
\hline & 1 & $\mathrm{~A}$ & $\mathrm{~B}$ \\
\hline \hline acima & 0 & 0 & 0 \\
\hline meio & 0 & $\left.\left.\mathcal{S}_{2}(i, l)\right|_{g_{m}=0} \mathcal{S}_{2}(k, j)\right|_{g_{m}=0}$ & $\left.\left.\mathcal{S}_{2}(i, k)\right|_{g_{m}=0} \mathcal{S}_{2}(j, l)\right|_{g_{m}=0}$ \\
\hline abaixo & 0 & 0 & 0 \\
\hline
\end{tabular}

(b) $\left.\partial_{g_{m}} \mathcal{D}(i, j, k, l)\right|_{g_{m}=0}$ toma os seguintes valores:

$$
\begin{aligned}
\left.\sum_{\mathbf{x} \in \mathbb{Z}^{d}} \mathcal{S}_{4}\left(i_{k_{1}}, \ldots, \widehat{i_{k_{r}}}, \ldots, i_{k_{4}},(m, \mathrm{x})\right)\right|_{g_{m}=0} & \left.\mathcal{S}_{2}\left((m+1, \mathrm{x}), i_{k_{r}}\right)\right|_{g_{m}=0} \\
& -\left.\left.\mathcal{S}_{2}\left(i_{k_{1}}, i_{k_{2}}\right)\right|_{g_{m}=0} \partial_{g_{m}} \mathcal{S}_{2}\left(i_{k_{3}}, i_{k_{4}}\right)\right|_{g_{m}=0}
\end{aligned}
$$

na parte de cima de qualquer configuração com $i_{k_{r}} \in\left\{i_{k_{3}}, i_{k_{4}}\right\}$ e $\left(i_{k_{r}}\right)_{0}=$ $\max \left\{i_{0}, j_{0}, k_{0}, l_{0}\right\}, e$

$$
\begin{aligned}
\left.\sum_{\mathbf{x} \in \mathbb{Z}^{d}} \mathcal{S}_{2}\left(i_{k_{r}},(m, \mathbf{x})\right)\right|_{g_{m}=0} \mathcal{S}_{4}((m+1, \mathbf{x}) & \left., i_{k_{1}}, \ldots, \widehat{i_{k_{r}}}, \ldots, i_{k_{4}}\right)\left.\right|_{g_{m}=0} \\
& -\left.\left.\partial_{g_{m}} \mathcal{S}_{2}\left(i_{k_{1}}, i_{k_{2}}\right)\right|_{g_{m}=0} \mathcal{S}_{2}\left(i_{k_{3}}, i_{k_{4}}\right)\right|_{g_{m}=0}
\end{aligned}
$$

na parte de baixo de qualquer configuração com $i_{k_{r}} \in\left\{i_{k_{1}}, i_{k_{2}}\right\}$ e $\left(i_{k_{r}}\right)_{0}$ $=\min \left\{i_{0}, j_{0}, k_{0}, l_{0}\right\}$. 
(c) No meio da configuração 1 tem-se:

$$
\begin{aligned}
\partial_{g_{m}}^{2} \mathcal{D} & \left.(i, j, k, l)\right|_{g_{m}=0} \\
& =\left.\left.\sum_{\mathrm{x}, \mathrm{y} \in \mathbb{Z}^{d}} \mathcal{D}(i, j,(m, \mathrm{x}),(m, \mathrm{y}))\right|_{g_{m}=0} \mathcal{D}((m+1, \mathrm{x}),(m+1, \mathrm{y}), k, l)\right|_{g_{m}=0} .
\end{aligned}
$$

(d) As derivadas de ordem par são nulas nas bordas de qualquer configuração.

(e) As derivadas de ordem impar são nulas no meio e fora de qualquer configuração.

Demonstração: É conseqüência direta de 6.2.1 e 6.2.2. Para a parte (c), usando

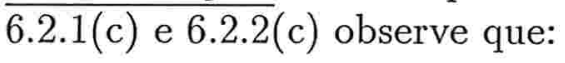

$$
\begin{aligned}
& \left.\partial_{g_{m}}^{2} \mathcal{D}(i, j, k, l)\right|_{g_{m}=0} \\
& =\left.\left.\sum_{\mathbf{x}, \mathbf{y} \in \mathbb{Z}^{d}} \mathcal{S}_{4}(i, j,(m, \mathbf{x}),(m, \mathbf{y}))\right|_{g_{m}=0} \mathcal{S}_{4}((m+1, \mathbf{x}),(m+1, \mathbf{y}), k, l)\right|_{g_{m}=0} \\
& -\left.\left[\left.\left.\sum_{\mathrm{x}, \mathrm{y} \in \mathbb{Z}^{d}} \mathcal{S}_{4}(i, j,(m, \mathrm{x}),(m, \mathrm{y}))\right|_{g_{m}=0} \mathcal{S}_{2}((m+1, \mathrm{x}),(m+1, \mathrm{y}))\right|_{g_{m}=0}\right] \mathcal{S}_{2}(k, l)\right|_{g_{m}=0} \\
& -\left.\left.\left.\mathcal{S}_{2}(i, j)\right|_{g_{m}=0} \sum_{\mathbf{x}, \mathbf{y} \in \mathbb{Z}^{d}} \mathcal{S}_{2}((m, \mathbf{x}),(m, \mathbf{y}))\right|_{g_{m}=0} \mathcal{S}_{4}((m+1, \mathrm{x}),(m+1, \mathrm{y}), k, l)\right|_{g_{m}=0} \\
& +\left.\left.\mathcal{S}_{2}(i, j)\right|_{g_{m}=0} \mathcal{S}_{2}(k, l)\right|_{g_{m}=0} \delta(m) \\
& =\left.\sum_{\mathbf{x}, \mathbf{y} \in \mathbb{Z}^{d}} \mathcal{S}_{4}(i, j,(m, \mathrm{x}),(m, \mathrm{y}))\right|_{g_{m}=0}\left[\left.\mathcal{S}_{4}((m+1, \mathrm{x}),(m+1, \mathrm{y}), k, l)\right|_{g_{m}=0}\right. \\
& \text { - } \left.\left.\left.\mathcal{S}_{2}((m+1, \mathbf{x}),(m+1, \mathrm{y}))\right|_{g_{m}=0} \mathcal{S}_{2}(k, l)\right|_{g_{m}=0}\right] \\
& -\left.\left.\sum_{\mathrm{x}, \mathrm{y} \in \mathbb{Z}^{d}} \mathcal{S}_{2}(i, j)\right|_{g_{m}=0} \mathcal{S}_{2}((m, \mathrm{x}),(m, \mathrm{y}))\right|_{g_{m}=0}\left[\left.\mathcal{S}_{4}((m+1, \mathrm{x}),(m+1, \mathrm{y}), k, l)\right|_{g_{m}=0}\right. \\
& \left.-\left.\left.\mathcal{S}_{2}((m+1, \mathrm{x}),(m+1, \mathrm{y}))\right|_{g_{m}=0} \mathcal{S}_{2}(k, l)\right|_{g_{m}=0}\right] \\
& =\left.\left.\sum_{\mathbf{x}, \mathbf{y} \in \mathbb{Z}^{d}} \mathcal{D}(i, j,(m, \mathrm{x}),(m, \mathrm{y}))\right|_{g_{m}=0} \mathcal{D}((m+1, \mathrm{x}),(m+1, \mathrm{y}), k, l)\right|_{g_{m}=0} \text {. }
\end{aligned}
$$


Dado $m \in \mathbb{Z}$, definimos agora os seguintes subespaços:

$$
\begin{aligned}
& \ell_{>m}^{2}\left(\mathbb{Z}^{d+1} \times \mathbb{Z}^{d+1}\right):=\left\{\left\{a_{i j}\right\}_{i, j \in \mathbb{Z}^{d+1}} \in \ell^{2}\left(\mathbb{Z}^{d+1} \times \mathbb{Z}^{d+1}\right): a_{i j}=0 \text { se } i_{0} \text { ou } j_{0} \leqslant m\right\} \\
& \ell_{\leqslant m}^{2}\left(\mathbb{Z}^{d+1} \times \mathbb{Z}^{d+1}\right):=\left\{\left\{a_{i j}\right\}_{i, j \in \mathbb{Z}^{d+1}} \in \ell^{2}\left(\mathbb{Z}^{d+1} \times \mathbb{Z}^{d+1}\right): a_{i j}=0 \text { se } i_{0} \text { ou } j_{0}>m\right\} \\
& \ell_{\div m}^{2}\left(\mathbb{Z}^{d+1} \times \mathbb{Z}^{d+1}\right):=\ell^{2}\left(\mathbb{Z}^{d+1} \times \mathbb{Z}^{d+1}\right) \ominus \ell_{>m}^{2}\left(\mathbb{Z}^{d+1} \times \mathbb{Z}^{d+1}\right) \ominus \ell_{\leqslant m}^{2}\left(\mathbb{Z}^{d+1} \times \mathbb{Z}^{d+1}\right) .
\end{aligned}
$$

\subsubsection{Lema:}

(a) Cada um dos subespaços acima definidos é invariante por $\mathcal{D}$, e portanto também por $\mathcal{D}^{-1}$.

(b) $E m \ell_{\div m}^{2}\left(\mathbb{Z}^{d+1} \times \mathbb{Z}^{d+1}\right) \cap \ell_{s}^{2}\left(\mathbb{Z}^{d+1} \times \mathbb{Z}^{d+1}\right)$ tem-se que:

$$
\left.\mathcal{D}^{-1}(i, j, k, l)\right|_{g_{m}=0}=\frac{1}{4}\left[\left.\left.\Gamma(i, k)\right|_{g_{m}=0} \Gamma(j, l)\right|_{g_{m}=0}+\left.\left.\Gamma(i, l)\right|_{g_{m}=0} \Gamma(j, k)\right|_{g_{m}=0}\right] .
$$

(c) No meio da configuraçẫo 1 tem-se:

$$
\left.\partial_{g_{m}} \mathcal{D} \cdot \mathcal{D}^{-1} \cdot \partial_{g_{m}} \mathcal{D}(i, j, k, l)\right|_{g_{m}=0}=\left.\partial_{g_{m}}^{2} \mathcal{D}(i, j, k, l)\right|_{g_{m}=0}
$$

Demonstração: A parte (a) segue de 6.3.1(a) analogamente a 5.1.5(a). A parte (b) pode ser provada substituindo diretamente as expressões envolvidas (usando 6.3.1(a)), porém resulta mais instrutiva a seguinte prova "construtiva":

Seja $A:=\left\{a_{k l}\right\}_{k, l \in \mathbb{Z}^{d+1}} \in \ell_{\dot{\div} m}^{2}\left(\mathbb{Z}^{d+1} \times \mathbb{Z}^{d+1}\right) \cap \ell_{s}^{2}\left(\mathbb{Z}^{d+1} \times \mathbb{Z}^{d+1}\right)$, e seja $\mathcal{A}$ um operador em $\ell_{\leftarrow m}^{2}\left(\mathbb{Z}^{d+1} \times \mathbb{Z}^{d+1}\right)$ não especificado pelo momento. Temos:

$$
\left(\left.\mathcal{A D}\right|_{g_{m}=0} A\right)_{i j}=\left.\left.\sum_{a, b \in \mathbb{Z}^{d+1}} \sum_{k, l \in \mathbb{Z}^{d+1}} \mathcal{A}(i, j, a, b)\right|_{g_{m}=0} \mathcal{D}(a, b, k, l)\right|_{g_{m}=0} a_{k l}
$$

Agora, pela invariância de $\mathcal{D}$ temos que o membro esquerdo acima é nulo a menos que o hiperplano $L_{m}$ separe o par $\{i, j\}$. Para fixar idéias, vamos supor que $j$ está por cima e que $i$ está em ou abaixo de $L_{m}$. Por outra parte, pela hipótese sobre $A$, cada somando no membro direito acima é nulo a menos que $L_{m}$ separe o par $\{k, l\}$. Mas então, 6.3.1(a) implica que o segundo fator na somatória é nulo a menos que $L_{m}$ separe o par $\{a, b\}$. Isto dá origem a quatro casos concernentes às posições dos pares $\{k, l\}$ e $\{a, b\}$ relativas ao hiperplano $L_{m}$. Supondo, por exemplo, que $l$ esteja por cima e $k$ em ou abaixo, temos que, para o caso em que $b$ está por cima e $a$ em ou abaixo, se substituimos $\mathcal{A}(i, j, a, b)$ por $\Gamma(i, a) \Gamma(j, b)$ e usamos 6.3.1(a) o membro direito acima vale $a_{i j}$ (observe que neste caso os sítios $a, b, k, l$ determinam 
uma configuração de tipo B). Para o caso em que $a$ está agora por cima e $b$ em ou abaixo, obtemos analogamente o mesmo resultado se substituimos $\mathcal{A}(i, j, a, b)$ por $\Gamma(i, b) \Gamma(j, a)$ (observe que neste caso os sítios $a, b, k, l$ também determinam uma configuração de tipo B). Observe agora que idênticos resultados obteriam-se substituindo $\mathcal{A}(i, j, a, b)$ em ambos casos por $\Gamma(i, a) \Gamma(j, b)+\Gamma(i, b) \Gamma(j, a)$ pois no primeiro caso o segundo somando é nulo e vice-versa. Os outros casos são análogos (nestes se usa a simetria do $A$ ). Assim, como em cada um destes quatro casos obtém-se $a_{i j}$, segue o estabelecido no enunciado. $O$ caso com $i$ por cima e $j$ em ou abaixo conduz analogamente ao mesmo resultado.

Para a parte (c), temos:

$$
\begin{aligned}
\left.\partial_{g_{m}} \mathcal{D} \cdot \mathcal{D}^{-1} \cdot \partial_{g_{m}} \mathcal{D}\right|_{g_{m}=0}(i, j, k, l) \\
\quad=\left.\left.\left.\sum_{a, b, c, d \in \mathbb{Z}^{d+1}} \partial_{g_{m}} \mathcal{D}(i, j, a, b)\right|_{g_{m}=0} \mathcal{D}^{-1}(a, b, c, d)\right|_{g_{m}=0} \partial_{g_{m}} \mathcal{D}(c, d, k, l)\right|_{g_{m}=0} .
\end{aligned}
$$

Observe que por 6.3.1(e), o terceiro fator na somatória à direita acima visto como função apenas dos índices $c, d$ está em $\ell_{\leftarrow m}^{2}\left(\mathbb{Z}^{d+1} \times \mathbb{Z}^{d+1}\right)$. Assim, cada somando será nulo a menos que $L_{m}$ separe o par $\{c, d\}$. Também, no meio da configuração 1 , por 6.3.1(e) o primeiro fator será nulo a menos que $L_{m}$ separe o par $\{a, b\}$. Isto dá origem a quatro casos concernentes às posições dos pares $\{c, d\}$ e $\{a, b\}$ relativas ao hiperplano $L_{m}$. Vamos supor, para fixar idéias, que $b$ está por cima e $a$ em ou abaixo de $L_{m}$ e que $d$ está por cima e $c$ em ou abaixo de $L_{m}$. Observe-se que qualquer seja o caso, os sítios $i, j, a, b$ determinam configurações com $a$ ou $b$ no topo, e os sítios $c, d, k, l$ determinam configurações com $c$ ou $d$ no fundo. Usando 6.3.1(b) e a parte (b) já provada (observe que neste caso o segundo somando em 6.3.2(b) é 
nulo), temos:

$$
\begin{aligned}
& \left.\partial_{g_{m}} \mathcal{D} \cdot \mathcal{D}^{-1} \cdot \partial_{g_{m}} \mathcal{D}\right|_{g_{m}=0}(i, j, k, l) \\
& =\left.\left.\left.\sum_{a, b, c, d \in \mathbb{Z}^{d+1}} \partial_{g_{m}} \mathcal{D}(i, j, a, b)\right|_{g_{m}=0} \mathcal{D}^{-1}(a, b, c, d)\right|_{g_{m}=0} \partial_{g_{m}} \mathcal{D}(c, d, k, l)\right|_{g_{m}=0} \\
& =\left.\left.\left.\frac{1}{4} \sum_{a, b, c, d \in \mathbb{Z}^{d+1}} \sum_{\mathbf{x}, \mathbf{y} \in \mathbb{Z}^{d}} \mathcal{S}_{4}(i, j, a,(m, \mathrm{x}))\right|_{g_{m}=0} \mathcal{S}_{2}((m+1, \mathrm{x}), b)\right|_{g_{m}=0} \Gamma(a, c)\right|_{g_{m}=0} \\
& \times\left.\left.\left.\Gamma(b, d)\right|_{g_{m}=0} \mathcal{S}_{2}(c,(m, \mathrm{y}))\right|_{g_{m}=0} \mathcal{S}_{4}((m+1, \mathrm{y}), d, k, l)\right|_{g_{m}=0} \\
& -\left.\left.\left.\frac{1}{4} \sum_{a, b, c, d \in \mathbb{Z}^{d+1}} \sum_{\mathbf{x}, \mathbf{y} \in \mathbb{Z}^{d}} \mathcal{S}_{4}(i, j, a,(m, \mathrm{x}))\right|_{g_{m}=0} \mathcal{S}_{2}((m+1, \mathrm{x}), b)\right|_{g_{m}=0} \Gamma(a, c)\right|_{g_{m}=0} \\
& -\left.\left.\left.\left.\frac{1}{4} \sum_{a, b, c, d \in \mathbb{Z}^{d+1}} \sum_{\mathbf{y} \in \mathbb{Z}^{d}} \mathcal{S}_{2}(i, j)\right|_{g_{m}=0} \partial_{g_{m}} \mathcal{S}_{2}(a, b)\right|_{g_{m}=0} \Gamma(a, c)\right|_{g_{m}=0} \Gamma(b, d)\right|_{g_{m}=0} \\
& \times\left.\left.\frac{1}{4} \mathcal{S}_{a, b, c, d \in \mathbb{Z}^{d+1}}(c,(m, \mathrm{y}))\right|_{g_{m}=0} \mathcal{S}_{4}((m+1, \mathrm{y}), d, k, l)\right|_{g_{m}=0} \\
& \left.\left.\left.\left.\sum_{\mathbf{y} \in \mathbb{Z}^{d}} \mathcal{S}_{2}(i, j)\right|_{g_{m}=0} \partial_{g_{m}} \mathcal{S}_{2}(a, b)\right|_{g_{m}=0} \Gamma(a, c)\right|_{g_{m}=0} \Gamma(b, d)\right|_{g_{m}=0} \\
& \times\left.\left.\partial_{g_{m}} \mathcal{S}_{2}(c, d)\right|_{g_{m}=0} \mathcal{S}_{2}(k, l)\right|_{g_{m}=0} .
\end{aligned}
$$

Usando agora 5.1.2(a) para substituir as derivadas das funções de 2-pontos nos últimos três somandos, obtem-se a expressão intermédiaria que aparece na prova de $6.3 .1(\mathrm{c})$ vezes o fator $1 / 4$. Os outros três casos são tratados analogamente e conduzem ao mesmo resultado.

\subsubsection{Lema:}

(a) $\left.\mathcal{D}^{-1}(i, j, k, l)\right|_{g_{m}=0}=0$ nas bordas de qualquer configuração e também no meio da 1 .

(b) Se $\left|i_{0}-j_{0}\right| \geqslant 2$ e $\left|k_{0}-l_{0}\right| \geqslant 2$, então $\left.\partial_{g_{m}} \mathcal{D}^{-1}(i, j, k, l)\right|_{g_{m}=0}=0$ nas partes de baixo $\left(\right.$ com $i_{0}<m$ ), meio, de cima (com $m<l_{0}-1$ ) e fora de qualquer configuração.

(c) $\left.\partial_{g_{m}}^{2} \mathcal{D}^{-1}(i, j, k, l)\right|_{g_{m}=0}=\left.\partial_{g_{m}}^{2} \mathcal{D}_{0}^{-1}(i, j, k, l)\right|_{g_{m}=0}$ no meio da configuração 1 .

Seja $n \in \mathbb{N} \cup\{0\}$. Então, 
(d) $n$ par $\left.\Longrightarrow \partial_{g_{m}}^{n} \mathcal{D}^{-1}(i, j, k, l)\right|_{g_{m}=0}=0$ nas bordas de qualquer configuração.

$$
\begin{aligned}
& n \text { impar } \Longrightarrow\left.\partial_{g_{m}}^{n} \mathcal{D}^{-1}(i, j, k, l)\right|_{g_{m}=0} \\
& \text { no meio e fora de qualquer configuração. }
\end{aligned}
$$

Demonstração: A parte (a) segue de 6.3.1(a), analogamente a 5.2.1(a). Uma vez provada a parte (b), a prova da parte (d) é análoga à 5.2.3. Portanto, a parte crucial do presente lema é provar (b). Para isto, observe que usando uma fórmula análoga à de 5.2.2 temos:

$$
\begin{aligned}
\partial_{g_{m}} & \left.\mathcal{D}^{-1}(i, j, k, l)\right|_{g_{m}=0} \\
& =-\left.\left.\left.\sum_{a, b, c, d \in \mathbb{Z}^{d+1}} \mathcal{D}^{-1}(i, j, a, b)\right|_{g_{m}=0} \partial_{g_{m}} \mathcal{D}(a, b, c, d)\right|_{g_{m}=0} \mathcal{D}^{-1}(c, d, k, l)\right|_{g_{m}=0}
\end{aligned}
$$

Consideremos primeiramente o caso de uma configuração $\operatorname{com} i_{0}=\min \left\{i_{0}, j_{0}, k_{0}, l_{0}\right\}$. Na parte de baixo, o primeiro fator é nulo, pela parte (a), a menos que o hiperplano $L_{m}$ separe o par $\{a, b\}$, e pelo mesmo motivo o terceiro fator é nulo a menos que o par $\{c, d\}$ esteja por acima de $L_{m}$. Assim, considerando o segundo fator, estamos na parte de baixo de alguma configuração que tem $a$ ou $b$ na parte de baixo. Observe-se que existem dois casos, concernentes às posições de $a$ e $b$ relativas a $L_{m}$. Vamos supor que $b$ esteja por cima e $a$ em ou abaixo de $L_{m}$ (o outro caso é análogo). Observe-se também que pelo antedito, o produto dos dois últimos fatores visto apenas como função de $a, b$ está em $\ell_{\div m}^{2}\left(\mathbb{Z}^{d+1} \times \mathbb{Z}^{d+1}\right)$. Usando então 6.3.2(b) para substituir o primeiro fator e 6.3.1(b) para o segundo, temos que o membro direito em (6.3.1) pode ser escrito como a soma de:

$$
\begin{aligned}
&-\left.\frac{1}{4} \sum_{a, b, c, d \in \mathbb{Z}^{d+1}} \sum_{\mathbf{x} \in \mathbb{Z}^{d}} \Gamma(i, a)\right|_{g_{m}=0}\left.\left.\Gamma(j, b)\right|_{g_{m}=0} \mathcal{S}_{2}(a,(m, \mathrm{x}))\right|_{g_{m}=0} \\
& \times \times\left.\mathcal{S}_{4}((m+1, \mathrm{x}), b, c, d)\right|_{g_{m}=0} \\
&=-\left.\left.\left.\frac{1}{4} \sum_{b, c, d \in \mathbb{Z}^{d+1}} \sum_{\mathbf{x} \in \mathbb{Z}^{d}} \delta_{i(m, \mathbf{x})} \Gamma(j, b)\right|_{g_{m}=0} \mathcal{S}_{4}((m+1, \mathrm{x}), b, c, d)\right|_{g_{m}=0} \mathcal{D}^{-1}(c, d, k, l)\right|_{g_{m}=0} \\
&=0 \text { se } i_{0}<m,
\end{aligned}
$$


mais

$$
\begin{aligned}
&\left.\left.\left.\frac{1}{4} \sum_{a, b, c, d \in \mathbb{Z}^{d+1}} \sum_{\mathbf{x} \in \mathbb{Z}^{d}} \Gamma(i, a)\right|_{g_{m}=0} \Gamma(j, b)\right|_{g_{m}=0} \mathcal{S}_{2}(a,(m, \mathrm{x}))\right|_{g_{m}=0} \\
& \times\left.\left.\left.\mathcal{S}_{2}((m+1, \mathrm{x}), b)\right|_{g_{m}=0} \mathcal{S}_{2}(c, d)\right|_{g_{m}=0} \mathcal{D}^{-1}(c, d, k, l)\right|_{g_{m}=0} \\
&=\left.\left.\frac{1}{4} \sum_{c, d \in \mathbb{Z}^{d+1}} \sum_{\mathbf{x} \in \mathbb{Z}^{d}} \delta_{i(m, \mathrm{x})} \delta_{j(m+1, \mathbf{x})} \mathcal{S}_{2}(c, d)\right|_{g_{m}=0} \mathcal{D}^{-1}(c, d, k, l)\right|_{g_{m}=0} \\
&=0 \text { se }\left|i_{0}-j_{0}\right| \geqslant 2 .
\end{aligned}
$$

Na parte do meio, distinguimos dois casos:

Caso 1: $j$ está em ou abaixo de $L_{m}$.

Pela parte (a), o primeiro fator em (6.3.1) é nulo a menos que o par $\{a, b\}$ esteja abaixo de $L_{m}$ e pelo mesmo motivo o terceiro fator é nulo a menos que o par $\{c, d\}$ esteja por cima de $L_{m}$. Mas então o segundo fator é nulo por 6.3.1(e).

Caso 2: $j$ está por cima de $L_{m}$.

Pela parte (a), o primeiro fator em (6.3.1) é nulo a menos que $L_{m}$ separe o par $\{a, b\}$ e pelo mesmo motivo o terceiro fator é nulo a menos que $L_{m}$ separe o par $\{c, d\}$. Mas então o segundo fator é nulo por 6.3.1(e).

Na parte de cima a análise é análoga à da parte de baixo.

$\mathrm{Na}$ região de fora inferior (resp. superior), pela parte (a) o primeiro e terceiro fatores são nulos a menos que os pares $\{a, b\}$ e $\{c, d\}$ estejam por cima (resp. por baixo) de $L_{m}$ mas neste caso o segundo fator tambén é nulo, por 6.3.1(e).

Os casos das outras configurações são análogos.

Para a parte (c), usando uma fórmula análoga à de 5.2.2, tem-se:

$$
\begin{aligned}
& \partial_{g_{m}}^{2} \mathcal{D}^{-1}=-\mathcal{D}^{-1} \cdot \partial_{g_{m}}^{2} \mathcal{D} \cdot \mathcal{D}^{-1}-2 \mathcal{D}^{-1} \cdot \partial_{g_{m}} \mathcal{D} \cdot \partial_{g_{m}} \mathcal{D}^{-1} \\
& =-\mathcal{D}^{-1} \cdot \partial_{g_{m}}^{2} \mathcal{D} \cdot \mathcal{D}^{-1}+2 \mathcal{D}^{-1} \cdot \partial_{g_{m}} \mathcal{D} \cdot \mathcal{D}^{-1} \cdot \partial_{g_{m}} \mathcal{D} \cdot \mathcal{D}^{-1} \\
& =-\mathcal{D}^{-1}\left(\partial_{g_{m}}^{2} \mathcal{D}-2 \partial_{g_{m}} \mathcal{D} \cdot \mathcal{D}^{-1} \cdot \partial_{g_{m}} \mathcal{D}\right) \mathcal{D}^{-1}
\end{aligned}
$$

Como na prova da parte (b), pela parte (a), cada somando é nulo a menos que os sítios $a, b, c, d$ determinem uma configuração de tipo $1 \mathrm{com} a$ e $b$ na parte de baixo. 
Avaliando em $g_{m}=0$ e usando 6.3.2(c) e 6.3.1(c) temos:

$$
\begin{aligned}
& \left.\partial_{g_{m}}^{2} \mathcal{D}^{-1}(i, j, k, l)\right|_{g_{m}=0} \\
& =\left.\left.\left.\sum_{a, b, c, d \in \mathbb{Z}^{d+1}} \mathcal{D}^{-1}(i, j, a, b)\right|_{g_{m}=0} \partial_{g_{m}}^{2} \mathcal{D}(a, b, c, d)\right|_{g_{m}=0} \mathcal{D}^{-1}(c, d, k, l)\right|_{g_{m}=0} \\
& =\left.\left.\sum_{a, b, c, d \in \mathbb{Z}^{d+1}} \sum_{\mathbf{x}, \mathbf{y} \in \mathbb{Z}^{d}} \mathcal{D}^{-1}(i, j, a, b)\right|_{g_{m}=0} \mathcal{D}(a, b,(m, \mathbf{x}),(m, \mathbf{y}))\right|_{g_{m}=0} \\
& \quad \times\left.\left.\mathcal{D}((m+1, \mathbf{x}),(m+1, \mathbf{y}), c, d)\right|_{g_{m}=0} \mathcal{D}^{-1}(c, d, k, l)\right|_{g_{m}=0} \\
& =\sum_{\mathbf{x}, \mathbf{y} \in \mathbb{Z}^{d}} \delta_{i(m, \mathbf{x})} \delta_{j(m, \mathbf{y})} \delta_{(m+1, \mathbf{x}) k} \delta_{(m+1, \mathbf{y}) l} \\
& =\left(-\sum_{\mathbf{x} \in \mathbb{Z}^{d}} \delta_{i(m, \mathbf{x})} \delta_{(m+1, \mathbf{x}) k}\right)\left(-\sum_{\mathbf{y} \in \mathbb{Z}^{d}} \delta_{j(m, \mathbf{y})} \delta_{(m+1, \mathbf{y}) l}\right) \\
& =\left.\left.\partial_{g_{m}} \Gamma(i, k)\right|_{g_{m}=0} \partial_{g_{m}} \Gamma(j, l)\right|_{g_{m}=0}=\left.\partial_{g_{m}}^{2} \mathcal{D}_{0}^{-1}(i, j, k, l)\right|_{g_{m}=0},
\end{aligned}
$$

onde na penúltima igualdade temos usado (5.2.1) e para a última 6.1.2(b) e 5.2.1(a).

\subsection{O Núcleo de Bethe-Salpeter}

\subsubsection{Lema:}

(a) Para $k=0,1,2,3$, no meio da configuração 1, tem-se:

$$
\left.\partial_{g_{m}}^{k} \mathcal{N}(i, j, k, l)\right|_{g_{m}=0}=0
$$

(b) Se $\left|i_{0}-j_{0}\right| \geqslant 2,\left|k_{0}-l_{0}\right| \geqslant 2$, então para $k=0,1,2$, nas bordas (com $i_{0}<m$ e $\left.m<l_{0}-1\right)$ de todas as configurações, tem-se:

$$
\left.\partial_{g_{m}}^{k} \mathcal{N}(i, j, k, l)\right|_{g_{m}=0}=0
$$

Demonstração: De 3.1 .2 , p. 32 , sabe-se que $\mathcal{N}=\mathcal{D}^{-1}-\mathcal{D}_{0}^{-1}$, e assim basta usar 6.1 .3 e 6.3 .3 . 
6.4.2 Corolário: No modelo de Ising na região de altas temperaturas tem-se:

$$
|\mathcal{N}(i, j, k, l)| \leqslant \text { const } \mathrm{e}^{-m\left(\left|i_{0}-j_{0}\right|+\left|k_{0}-l_{0}\right|+2\left|i_{0}+j_{0}-k_{0}-l_{0}\right|\right)} .
$$

Demonstraçấo: É análoga à de 5.1.3. Observe que:

$$
\left|i_{0}+j_{0}-k_{0}-l_{0}\right| \leqslant\left|i_{0}-j_{0}\right|+2\left|j_{0}-k_{0}\right|+\left|k_{0}-l_{0}\right|
$$

de onde:

$$
\begin{aligned}
& 3\left|i_{0}-j_{0}\right|+4\left|j_{0}-k_{0}\right|+3\left|k_{0}-l_{0}\right| \\
& =\left|i_{0}-j_{0}\right|+\left|k_{0}-l_{0}\right|+2\left(\left|i_{0}-j_{0}\right|+2\left|j_{0}-k_{0}\right|+\left|k_{0}-l_{0}\right|\right) \\
& \geqslant\left|i_{0}-j_{0}\right|+\left|k_{0}-l_{0}\right|+2\left|i_{0}+j_{0}-k_{0}-l_{0}\right| .
\end{aligned}
$$

Agora, observe-se que para $a, b \in \mathbb{R}$ quaisquer tem-se:

$$
|a-b| \leqslant|a-b-1|+1 \Longrightarrow|a-b-1| \geqslant|a-b|-1 \text {. }
$$

Para a configuração 1 , por 6.4.1(a) tem-se:

$$
\begin{aligned}
& 3\left|i_{0}-j_{0}-1\right|+4\left|j_{0}-k_{0}\right|+3\left|k_{0}-l_{0}-1\right| \\
& \geqslant 3\left|i_{0}-j_{0}\right|+4\left|j_{0}-k_{0}\right|+3\left|k_{0}-l_{0}\right|-6 \\
& \quad \geqslant\left|i_{0}-j_{0}\right|+\left|k_{0}-l_{0}\right|+2\left|i_{0}+j_{0}-k_{0}-l_{0}\right|-6 .
\end{aligned}
$$

Aqui, para a primeira desigualdade temos usado (6.4.2) e para a segunda (6.4.1).

6.4.3 Observação: O desacoplamento por hiperplanos, feito no presente capítulo para o caso de hiperplanos perpendiculares à direção $x_{0}$, pode ser feito analogamente para hiperplanos perpendiculares a qualquer outra direção. Desta maneira, o decaimento exponencial do núcleo de Bethe-Salpeter verificado em 6.4 .2 é válido em todas as outras coordenadas simultaneamente.

6.4.4 Lema: O núcleo de Bethe-Salpeter $K$ satisfaz a hipótese H2 da seção 3.4, p. 46 .

Demonstração: Com a seguinte mudança na notação da seção 3.2 , p. 34 :

$$
y_{1}=i \quad y_{2}=j \quad y_{3}=k \quad y_{4}=l,
$$


temos:

$$
\begin{aligned}
\tau^{\prime}=\tau-u_{1}-u_{3} & =i+j-k-l \\
u_{2} & =i-j \\
u_{4} & =k-l
\end{aligned}
$$

Usando 6.4.2, segue que:

$$
\left|\check{K}\left(\tau^{\prime}, u_{2}, u_{4}\right)\right|=|\mathcal{N}(i, j, k, l)| \leqslant \text { const } \mathrm{e}^{-2 m \tau^{\prime}-m u_{2}-m u_{4}} .
$$

Daqui, o resto é conseqüência de A.1 do apêndice no final da seção 5.1.

A técnica de desacoplamento por hiperplanos foi introduzida em modelos na rede por R. Schor, [84], [85], seguindo idéias usadas no contínuo por Spencer, [99]. É deste último trabalho que adaptamos a abordagem geral e certas idéias usadas nas provas da seção 6.3. Os resultados 5.1 .3 e 5.1 .4 são bem conhecidos, [93]. Em certos casos, o decaimento exponencial da função de 2 pontos (truncada) segue da convergência da expansão em polímeros, [37]. O caso do modelo de Ising na região de baixas temperaturas foi considerado em um trabalho seminal de R. Schor, [84]. Neste trabalho foi verificada a hipótese H1, p. 46, ou gap de massa superior e inferior, para a função de 2 pontos. Basicamente a mesma prova é válida para o caso de altas temperaturas, uma vez conhecido 5.2.4. Outras provas podem consultar-se em [69], [70]. O exponente 3 que aparece em 5.2.4, constitui uma melhora introduzida $^{1}$ em [69] com relacão ao exponente 2 da literatura anterior. Embora baseadas nos mesmos princípios gerais, grande parte das demonstrações aqui apresentadas são diferentes, considerando-se também o caso de derivadas superiores, independentemente da utilidade posterior que possam ter, pois nas nossas provas não existe economia nenhuma en restringir-se às primeiras. Dentre os trabalhos envolvendo o estudo de derivadas superiores destacamos [86], [87] e as referências deste último. Uma outra prova do decaimento do núcleo de Bethe-Salpeter para sistemas de spin ferromagnéticos clássicos na região de altas temperaturas pode consultar-se em um trabalho recente de R. Schor e M. O'Carroll. ${ }^{2}$

\footnotetext{
${ }^{1}$ Aparentemente, de maneira original.

${ }^{2}$ [89]. Veja-se também [88].
} 


\section{Parte III}

\section{Considerações Finais}




\section{Capítulo 7}

\section{Conclusão. Discussão e Comentários}

Nas páginas precedentes, o problema da completeza assintótica foi abordado com apenas uma ferramenta, e de caráter relativamente elementar, a saber, a teoria espectral de um operador autoadjunto em um espaço de Hilbert. É bem sabido que dado um tal operador, o de energia-momento em nosso caso, o espaço de Hilbert pode ser quebrado em:

$$
\mathcal{H}=\mathcal{H}_{d} \oplus \mathcal{H}_{a c} \oplus \mathcal{H}_{s c}
$$

segundo a medida espectral seja discreta, absolutamente contínua ou singular, respectivamente. Na nossa abordagem, estudamos a medida espectral, provando que $\mathcal{H}_{d}$ provém basicamente dos polos de um certo operador integral. Exceto isso, o resto do espectro, até uma determinada energia, é absolutamente contínuo e de multiplicidade 1 , o que permite descartar $\mathcal{H}_{s c}$ e identificar $\mathcal{H}_{a c}$ com os estados assintóticos $\mathcal{H}_{\text {in }}$. A introdução de uma hipótese adequada permite descartar também a existência de polos, o que finalmente conduz à completeza assintótica, até essa determinada energia.

Fundamental para o estudo da medida espectral foi o resultado 3.3.1, representado simbolicamente pela identidade (3.3.3):

$$
\begin{aligned}
& \lim _{y \rightarrow 0^{+}} \int_{0}^{\infty} \operatorname{Im}\left\langle f, R\left(\left(k_{0}, \mathrm{k}\right)\right) g\right\rangle h(x) d x \\
& =(2 \pi)^{\frac{d+1}{2}} \pi \int_{0}^{\infty} h(a) \sqrt{(a+1)^{2}-1} d\left\langle\Theta(\check{f}), E_{(2 \operatorname{arcosh}(a+1),-2 \mathbf{k})} \Theta(\check{g})\right\rangle_{\mathcal{H}} .
\end{aligned}
$$

Antes de estabelecer esta identidade, foi introduzido o espaço de Hilbert $\mathcal{H}$ (seção 2.1) e a equação de Bethe-Salpeter na rede (seção 3.2). Resulta digno de destacar 
que a identidade (7.1) não depende do modelo nem de nenhuma hipótese especial sobre as funções de $n$ pontos. Aqui k é arbitrário mas fixo. Assim, este método não permite estudar o espectro conjunto do operador de energia-momento, senão o espectro de energia com momento (arbitrário, em principio, porém) fixo. Esta identidade é válida apenas para os "estados de duas partículas":

$$
\Theta(\check{f})=\sum_{\mathbf{x} \in \mathbb{Z}^{d}} \check{f}(-\mathrm{x}) \mathrm{e}^{-\frac{1}{2} i \mathbf{x} \cdot \mathbf{P}}\left(P_{(0, \mathbf{x})} P_{0}-\mu\left(p_{(0, \mathbf{x})} p_{0}\right) I_{\mathcal{H}}\right) \Omega,
$$

permitindo desta maneira obter informação apenas na parte de baixas energias do espectro. Esta limitação é intrínseca ao fato de ter usado apenas a informação contida na função de 4 pontos, da qual $R$ é basicamente a sua transformada de Fourier, constituindo-se $a b$ initio em uma abordagem incompleta da teoria. ${ }^{1} \mathrm{O}$ estudo do espectro para energias maiores requer o uso de núcleos de ordem superior. ${ }^{2}$ Mais específicamente, o que é estudado é o espectro do operador de energia-momento restrito ao subespaço:

$$
\overline{\operatorname{span}}\left\{\Theta(\check{f}): f \in A_{\delta}\right\} .
$$

A questão da convergência da série em (7.2) pode ser contornada observando que:

$$
\begin{aligned}
& \overline{\operatorname{span}}\left\{\Theta(\check{f}): f \in A_{\delta}^{1} \wedge \operatorname{supp} \check{f} \text { é compacto }\right\} \\
& \quad=\overline{\operatorname{span}}\left\{\Theta(\check{f}): f \in A_{\delta}^{1}\right\}=\overline{\operatorname{span}}\left\{\Theta(\check{f}): f \in A_{\delta}\right\} .
\end{aligned}
$$

O membro esquerdo em (7.1) define uma distribuição em $\mathcal{D}(0,+\infty)$, envolvendo a família de operadores $R(k)$, onde $k$ é um parâmetro complexo. Este operador é dado por:

$$
R(k)=R_{0}\left[I+K(k) R_{0}(k)\right]^{-1}
$$

desde que o inverso no segundo fator à direita acima exista. Aqui $K(k)$ é o núcleo de Bethe-Salpeter, e $R_{0}(k)$ é um operador integral, que agindo sobre funções simétricas está dado pelo núcleo (3.2.8):

$$
R_{0}(k, p, q)=2(2 \pi)^{\frac{d+1}{2}} \widehat{S}_{2}(k+p) \widehat{S}_{2}(k-p) \delta(p+q),
$$

e onde a função de 2 pontos supõe-se estar dada segundo a hipótese H1:

$$
\widehat{S}_{2}\left(p_{0}, \mathbf{p}\right)=Z(\mathbf{p}) \operatorname{Re}\left(\frac{1+\mathrm{e}^{-\left(\omega(\mathbf{p})+i p_{0}\right)}}{1-\mathrm{e}^{-\left(\omega(\mathbf{p})+i p_{0}\right)}}\right)+\int_{m+2 \delta_{0}}^{\infty} \operatorname{Re}\left(\frac{1+\mathrm{e}^{-\left(\lambda_{0}+i p_{0}\right)}}{1-\mathrm{e}^{-\left(\lambda_{0}+i p_{0}\right)}}\right) d \rho\left(\lambda_{0}, \mathbf{p}\right) .
$$

Dentre as diversas circunstâncias em que o operador em (7.3) é invertível, destacamos as seguintes duas condições suficientes:

\footnotetext{
${ }^{1}$ No Teorema de Reconstrução de Wightman,v.g., a teoria é reconstruida usando a seqüência completa das distribuições de Wightman, e não apenas as primeiras.

${ }^{2}$ [21], [68]. Núcleos de ordem superior são considerados detalhadamente em [20]; Cf. [32].
} 
(a) A norma de $K(k) R_{0}(k)$ é pequena.

(b) $K(k) R_{0}(k)$ é uma família analítica de operadores compactos.

A segunda condição é "quase suficiente", no sentido que garante a existência do inverso salvo um conjunto discreto contido na região de analiticidade. ${ }^{3}$ Este conjunto é denominado conjunto de polos do operador. Existe uma outra condição que, salvo a analiticidade, é suficiente para as duas anteriores:

(c) A norma de Hilbert-Schmidt de $K(k) R_{0}(k)$ é pequena.

Esta última condição é também particularmente apropriada para o contexto de operadores integrais no qual trabalhamos. Em 3.4.5 verificamos as condições (b) e (c) para $K\left(k_{0}, 0\right) R_{0}\left(k_{0}, 0\right)$ com $k_{0}$ na região:

$$
D_{1}:=\left\{\left|\operatorname{Re} k_{0}\right|<\pi / 2 \wedge\left|\operatorname{Im} k_{0}\right|<m+\delta_{0}\right\} \backslash\left\{\operatorname{Re} k_{0}=0 \wedge m \leqslant\left|\operatorname{Im} k_{0}\right|<m+\delta_{0}\right\} .
$$

Aqui foi usada fortemente a hipótese $\mathrm{H} 2$ sobre o decaimento exponencial do núcleo de Bethe-Salpeter. É bom salientar que quase toda a prova está contida nos resultados preliminares 3.4.1, 3.4.2 e 3.4.4.

Provados estes fatos sobre $K R_{0}$, partimos para o estudo da distribuição definida pelo membro esquerdo de (7.1). O resultado 3.5.2 afirma básicamente que o espectro do operador de energia momento até $2 m$ está contido nos polos de $R$. Uma vez introduzida a hipótese extra $\mathrm{H} 3$, foi possível descartar a existência desses estados ligados (lema 3.6.1), verificando a condição (c). É bom salientar que a parte crucial deste resultado consiste em que a mesma constante a serve uniformemente para todos os $k_{0}$ na região indicada. A existência de constantes $a=a\left(k_{0}\right)$ que satisfazem:

$$
\left\|K_{1}\left(k_{0}, 0\right) R_{0}^{\prime}\left(k_{0}, 0\right)\right\|_{H-S} \leqslant c(k)+o\left(a^{-1}\right)
$$

para determinados $k_{0}$, nesta altura é um fato quase trivial e que está contido implicitamente nas provas dos resultados da seção 3.4 (particularmente 3.4 .2 e 3.4.5). Mas isto último não basta, pois $a$ é um parámetro do modelo, que deve ser mantido constante. Os resultados apresentados até a seção 3.5 inclusive, gozam de razoável generalidade e certa independência do modelo. Já os da seção 3.6 revelam dependência do modelo, pois a hipótese H3 é uma paráfrase da condição ${ }^{* *}$ ) introduzida em [101] p. 5, que é específica das interações $P(\varphi)_{2}$ aí consideradas.

A informação sobre a parte absolutamente contínua do espectro envolve certa perda de generalidade, pois foi necesário restringir-se ao caso $d=1$. Isto permite

\footnotetext{
${ }^{3}$ Cf. Lemma 13 em [27] p. 592.
} 
"inverter" a relação de dispersão para obter uma derivada de Radon-Nikodym da medida espectral (lema 3.7.2) provando que o espectro contido em $\left[2 m, 2\left(m+\delta_{1}\right)\right)$ é absolutamente contínuo e de multiplicidade 1 (lema 3.7.5). Aqui, $\delta_{1}$ é uma constante $0<\delta_{1} \leqslant \delta_{0}$ "bem pequena" (Cf. 3.6.1).

Mas já que a restrição a dimensão $1+1$ foi aparentemente inevitável, podemos tirar proveito desta limitação, se cabe a expressão, introduzindo a suposição S1 sobre a relação de dispersão, que possibilita ampliar a região da completeza até $\delta_{1}=\delta_{0}$, deslocando o corte na região de analiticidade (lemas 3.7.1 e 3.7.4). A suposição S1 no caso $\omega\left(p_{1}\right)=\sqrt{p_{1}^{2}+4 m^{2}}$ está exemplificada em [101], e o mesmo caminho serve no caso do "campo livre aproximado" $\omega_{0}\left(p_{1}\right)=a+b p_{1}^{2} \cdot{ }^{4}$ Como já foi dito, a condição S1 foi estabelecida tendo o exemplo de Spencer e Zirilli como paradigmal. O conhecimento explícito da relação de dispersão constitui um ajuda, aparentemente quase imprescindível, na determinação do caminho $t(s)$.

Neste olhar retrospectivo, parece oportuno colocar a seguinte questão: É a completeza assintótica um problema demasiado complexo para ser abordado com esta ferramenta tão simples ou, pelo contrário, trata-se no fundo de um assunto simples no qual a complexidade reside em outro lugar? Qualquer que seja o caso, o alcance dos métodos apresentados é restrito por limitações metodológicas, ou seja, inerentes ao método empregado, presentes $a b$ initio no formalismo da teoria e aparentemente impossíveis de contornar sem trocar de método, a saber:

- Não nos é acessível o espectro conjunto do operador de energia-momento, mas o espectro de energia com momento fixo (em princípio, arbitrário).

- O próprio operador de energia-momento também não nos é acessível, mas a sua restrição ao subespaço de "estados de duas partículas", o que conduz à completeza assintótica apenas na região de baixas energias.

\footnotetext{
${ }^{4}$ Cf. seção 2.2 , p. 23.
} 


\title{
Capítulo 8
}

\section{Perspectivas, Conjeturas, Divagação}

\author{
Any formal manipulations that are not obiously wrong are assumed to be \\ correct. \\ M. L. Goldberger \& K. Watson ${ }^{1}$
}

Na teoria espectral de um operador auto-adjunto $A$ (não necessariamente limitado) em um espaço de Hilbert $\mathcal{H}$, a medida espectral é um ente derivado, revelando-se as propriedades analíticas do resolvente como conceito mais primitivo. ${ }^{2}$ Assim considerado, o estudo da distribuição no membro direito de (7.1) não constitui de maneira alguma novidade. Aliás, existem resultados ligando diretamente características do espectro às propriedades analíticas da função resolvente $R(\lambda)=(A-\lambda I)^{-1}$. Se $\lambda_{0}$ é um autovalor isolado, $R(\lambda)$ tem um polo em $\lambda_{0}$ (como $A$ é auto-adjunto, $\lambda_{0}$ é real e o polo é simples). Quando $v \in \mathcal{H}_{d}$, i.e., $v$ é uma combinação linear de autovetores, então $\|R(\lambda) v\|$ diverge como const $|\operatorname{Im} \lambda|^{-1}$ quando $\operatorname{Im} \lambda \rightarrow 0$ para algum valor de $\operatorname{Re} \lambda$ no espectro pontual. Quando $v \in \mathcal{H} \ominus \mathcal{H}_{d}$, espera-se que $\|R(\lambda) v\|$ divirja menos rapidamente, se bem que tal rapidez dependeria do fato de $v \in \mathcal{H}_{a c}$ ou não. Considere-se o conjunto $M$ dos vetores $v$ para os quais existe uma constante $c(v)$ tal que

$$
\|R(\lambda) v\| \leqslant c(v)|\operatorname{Im} \lambda|^{-1 / 2} \forall \lambda .
$$

Foi provado ${ }^{3}$ que $M \subseteq \mathcal{H}_{a c}$ e, de fato, $\mathcal{H}_{a c}=\bar{M}$. Assim, este tipo de propriedades analíticas do resolvente dispensa, em princípio, a procura de uma derivada de Radon-Nikodym para a medida espectral no estudo do espectro absolutamente

\footnotetext{
${ }^{1}$ Citado em [78] p. 96.

${ }^{2}$ Cf. [79] p. 165 et ss..

${ }^{3}[41]$. Cf. [79] p. 233.
} 
contínuo. Uma abordagem baseada nestas considerações seja talvez a mais adequada para a generalização dos resultados da seção 3.7 em dimensões superiores.

Com respeito à parte II, chama a atenção que em não poucas circunstâncias as derivadas de $\mathcal{D}^{-1}$ e $\mathcal{D}_{0}^{-1}$ anulam-se separadamente. Surge naturalmente a questão de saber se derivadas superiores à segunda anulam-se pela substração das funções inversas acima. Porém, derivadas de ordem superior são bem mais complicadas de analisar. Por exemplo, a derivada terceira é dada por:

$$
\begin{aligned}
& \left.\partial^{3} \mathcal{S}_{n}\left(i_{1}, \ldots, i_{n}\right)\right|_{g_{m}=0}=\left.2 \sum_{\mathbf{x}} \mathcal{S}_{n+2}\left(i_{1}, \ldots, i_{n},(m, \mathbf{x}),(m+1, \mathbf{x})\right)\right|_{g_{m}=0} \\
& +\sum_{\mathbf{x}, \mathbf{y}, \mathbf{z}} \mathcal{S}_{n+6}\left(i_{1}, \ldots, i_{n},(m, \mathbf{x}),(m+1, \mathbf{x})\right. \\
& -3\left(\left.\sum_{\mathbf{x}} \mathcal{S}_{n+2}\left(i_{1}, \ldots, i_{n},(m, \mathbf{x}),(m+1, \mathbf{x})\right)\right|_{g_{m}=0}\right) \\
& \quad \times\left(\left.\sum_{\mathbf{y}, \mathbf{z}} \mathcal{S}_{4}((m, \mathbf{y}),(m+1, \mathbf{y}),(m, \mathbf{z}),(m+1, \mathbf{z}))\right|_{g_{m}=0}\right) \\
& -\left.\mathcal{S}_{n}\left(i_{1}, \ldots, i_{n}\right)\right|_{g_{m}=0} \\
& \quad \times\left(\left.\sum_{\mathbf{x}, \mathbf{y}, \mathbf{z}} \mathcal{S}_{6}((m, \mathbf{x}),(m+1, \mathbf{x}),(m, \mathbf{y}),(m+1, \mathbf{y}),(m, \mathbf{z}),(m+1, \mathbf{z}))\right|_{g_{m}=0}\right)
\end{aligned}
$$


que é pouco se comparada com os sete somandos da derivada quarta:

$$
\begin{aligned}
& \left.\partial^{4} \mathcal{S}_{n}\left(i_{1}, \ldots, i_{n}\right)\right|_{g_{m}=0} \\
& =\left.8 \sum_{\mathbf{x}, \mathbf{y}} \mathcal{S}_{n+4}\left(i_{1}, \ldots, i_{n},(m, \mathrm{x}),(m+1, \mathrm{x}),(m, \mathrm{y}),(m+1, \mathrm{y})\right)\right|_{g_{m}=0} \\
& -\left.\left.8 \mathcal{S}_{n}\left(i_{1}, \ldots, i_{n}\right)\right|_{g_{m}=0} \sum_{\mathbf{x}, \mathbf{y}} \mathcal{S}_{4}((m, \mathrm{x}),(m+1, \mathrm{x}),(m, \mathrm{y}),(m+1, \mathrm{y}))\right|_{g_{m}=0} \\
& +\sum_{\mathbf{x}, \mathbf{y}, \mathbf{z}, \mathbf{w}} \mathcal{S}_{n+8}\left(i_{1}, \ldots, i_{n},(m, \mathrm{x}),(m+1, \mathrm{x}),(m, \mathrm{y}),(m+1, \mathbf{y}),\right. \\
& (m, \mathrm{z}),(m+1, \mathrm{z}),(m, \mathrm{w}),(m+1, \mathrm{w}))\left.\right|_{g_{m}=0} \\
& -4\left(\left.\sum_{\mathrm{x}, \mathrm{y}} \mathcal{S}_{n+4}\left(i_{1}, \ldots, i_{n},(m, \mathrm{x}),(m+1, \mathrm{x}),(m, \mathrm{y}),(m+1, \mathrm{y})\right)\right|_{g_{m}=0}\right) \\
& \times\left(\left.\sum_{\mathbf{z}, \mathbf{w}} \mathcal{S}_{4}((m, \mathbf{z}),(m+1, \mathbf{z}),(m, \mathbf{w}),(m+1, \mathbf{w}))\right|_{g_{m}=0}\right) \\
& -4\left(\left.\sum_{\mathrm{x}} \mathcal{S}_{n+2}\left(i_{1}, \ldots, i_{n},(m, \mathrm{x}),(m+1, \mathrm{x})\right)\right|_{g_{m}=0}\right) \\
& \times\left(\left.\sum_{\mathbf{y}, \mathbf{z}, \mathbf{w}} \mathcal{S}_{6}((m, \mathbf{y}),(m+1, \mathbf{y})(m, \mathbf{z}),(m+1, \mathbf{z}),(m, \mathbf{w}),(m+1, \mathbf{w}))\right|_{g_{m}=0}\right) \\
& +\left.6 \mathcal{S}_{n}\left(i_{1}, \ldots, i_{n}\right)\right|_{g_{m}=0} \\
& \times\left(\left.\sum_{\mathbf{x}, \mathbf{y}} \mathcal{S}_{4}((m, \mathbf{x}),(m+1, \mathbf{x}),(m, \mathbf{y}),(m+1, \mathbf{y}))\right|_{g_{m}=0}\right) \\
& \times\left(\left.\sum_{\mathbf{z}, \mathbf{w}} \mathcal{S}_{4}((m, \mathbf{z}),(m+1, \mathbf{z}),(m, \mathbf{w}),(m+1, \mathbf{w}))\right|_{g_{m}=0}\right) \\
& -\left.\mathcal{S}_{n}\left(i_{1}, \ldots, i_{n}\right)\right|_{g_{m}=0} \\
& \times\left(\sum_{\mathbf{x}, \mathbf{y}, \mathbf{z}, \mathbf{w}} \mathcal{S}_{\mathcal{8}}((m, \mathbf{x}),(m+1, \mathbf{x}),(m, \mathbf{y}),(m+1, \mathbf{y}),\right. \\
& \left.(m, \mathbf{z}),(m+1, \mathbf{z}),(m, \mathbf{w}),(m+1, \mathbf{w}))\left.\right|_{g_{m}=0}\right) .
\end{aligned}
$$

Um outro modelo sucetível de estudo é o modelo de Ising na região de baixas temperaturas. Propriedades de decaimento da função de 2 pontos foram estudadas em [84]. O decaimento da função de 4 pontos talvez possa ser atacado ao longo das 
mesmas linhas, observando que também neste caso:

$$
\mathcal{D}(i, j, k, l)=\ll\left(\sigma_{i} \sigma_{j}-\sigma_{i}^{\prime} \sigma_{j}^{\prime}\right)\left(\sigma_{k} \sigma_{l}-\sigma_{k}^{\prime} \sigma_{l}^{\prime}\right) \gg .
$$

Encerra-se assim esta obra, nada mais do que um relatório sobre o atual estado de ignorância, principalmente do autor, no campo da completeza assintótica, e que por mais longe que esteja da medida dos seus desejos, entrega finalmente à imparcialidade dos entendidos. 


\section{Referências Bibliográficas}

[1] J. C. A. Barata, Scattering Theory for Euclidean Lattice Field Theories, PhD Thesis, F. U. Berlin, 1989.

[2] -, Reduction Formulae for Euclidean Lattice Theories, Comm. Math. Phys. 143 (1992), 545-558.

[3] - , S-Matrix Elements in Euclidean Lattice Theories, Rev. Math. Phys. Vol. 63 (1994), 497-513.

[4] — \& K. Fredenhagen, Particle Scattering in Euclidean Lattice Field Theories, Comm. Math. Phys. 138 (1991), 507-519.

[5] J. J. Bisognano \& E. H. Wichmann, On the Duality Condition for a Hermitian Scalar Field, J. Math. Phys. 16 (1975), 985-1007.

[6] — \& - On the Duality Condition for Quantum Fields, J. Math. Phys. 17 (1976), 303-321.

[7] N. N. Bogolubov, A. A. Logunov, A. I. Oksak \& I. T. Todorov, General Principles of Quantum Field Theory, Kluwer Academic, Dordrecht, 1990.

[8] H.-J. Borchers, On Structure of the Algebra of Fields Operators, Nuovo Cimento 24 (1962), 214-236.

[9] A. Bovier \& M. Zahradník, A Simple Inductive Approach to the Problem of Convergence of Cluster Expansions of Polymer Models, Jour. Stat. Phys. 100 (2000), 765-778.

[10] J. Bros, Analytic Structure of Green's Functions in Quantum Field Theory, in Mathematical Problems in Theoretical Physics (Lausanne, 1979), K. Osterwalder, editor. Lecture Notes in Physics 116, Springer-Verlag, Berlin, Heidelberg, New York, 1980. 
[11] — \& M. Lassalle, Analiticity Properties and Many-Particle Structure in General Quantum Field Theory II. One-Particle Irreducible $n$-Point Functions, Comm. Math. Phys. 43 (1975), 279-309.

[12] — \& - Analiticity Properties and Many-Particle Structure in General Quantum Field Theory III. Two-Particle Irreducible in a Single Channel, Comm. Math. Phys. 54 (1977), 33-62.

[13] S. G. Brush, History of the Lenz-Ising model, Rev. Mod. Phys. 39 (1967), 883-893.

[14] D. Buchholz, Collision Theory for Masseless Fermions, Comm. Math. Phys. 42 (1975), 269-279.

[15] - , Collision Theory for Masseless Bosons, Comm. Math. Phys. 52 (1977), 147-173.

[16] - , Quantum Field Theory of Masseless Particles and Scattering Theory, in Mathematical Problems in Theoretical Physics (Rome, 1977), G. Dell'Antonio, S. Doplicher \& G. Jona-Lasimio, editors. Lecture Notes in Physics 80, Springer-Verlag, Berlin, Heidelberg, 1978.

[17] - On Particles, Infraparticles, and the Problem of Asymptotic Completeness, in VIII-th International Congress on Mathematical Physics (Marseille, 1986), Mebkhout \& Sénéor, editors. World Scientific, Singapore, $198 ?$.

[18] - \& K. Fredenhagen, Locality and the Structure of Particle States, Comm. Math. Phys. 84 (1982), 1-54.

[19] C. Burnap, Isolated One Particle States in Boson Quantum Field Theory Models, Ann. Phys. 104 (1977), 184-196.

[20] M. Combescure \& F. Dunlop, $n$-Particle-Irreducible Functions in Euclidean Quantum Field Theory, Ann. Phys. 122 (1979), 102-150.

[21] — \& - Three Body Asymptotic Completeness for $P(\phi)_{2}$ Models, Comm. Math. Phys. 85 (1982), 381-418.

[22] J. B. Conway, Functions of One Complex Variable, Springer-Verlag, New York, 1973.

[23] -, A Course in Functional Analysis, Springer-Verlag, New York, $2^{\text {nd }}$ edition, 1990. 
[24] A. Cooper, J. Feldman \& L. Rosen, Legendre Transforms and $r$-Particle Irreducibility in Quantum Field Theory: The Formalism for $r=1,2$, Ann. Phys. 137 (1981), 146-209.

[25] J. Dimock \& J.-P. Eckmann, On the Bound State in Weakly Coupled $\lambda\left(\varphi^{6}-\right.$ $\left.\varphi^{4}\right)_{2}$, Comm. Math. Phys. 51 (1976), 41-54.

[26] — \& - Spectral Properties and Bound-State Scattering for Weakly Coupled $\lambda P(\phi)_{2}$ Models, Ann. Phys. 103 (1977), 289-314.

[27] N. Dunford \& J. T. Schwartz, Linear Operators, Part I: General Theory, Interscience, New York, 1958.

[28] P. A. Faria da Veiga, M. O'Carroll, E. Pereira \& R. Schor, Spectral Analysis of Weakly Coupled Stochastic Lattice Ginzburg-Landau Models, preprint?.

[29] R. Fernandez, J. Frölich \& A. D. Sokal, Random Walks, Critical Phenomena, and Triviality in Quantum Field Theory, Springer-Verlag, Berlin, Heidelberg, 1992.

[30] K. Fredenhagen, On the Existence of the Real Time Evolution in Euclidean Lattice Gauge Theories, Comm. Math. Phys. 101 (1985), 579-587.

[31] — \& M. Marcu, Charged States in $\mathbb{Z}_{2}$ Gauge Theories, Comm. Math. Phys. 92 (1983), 81-119.

[32] J. Glimm \& A. Jaffe, Particles and Bound States and Progress Toward Unitarity and Scaling, in International Symposium on Mathematical Problems in Theoretical Physics (Kyoto, 1975), H. Araki, editor. Lecture Notes in Physics 39, Springer-Verlag, Berlin, Heidelberg, New York, 1975.

[33] J. Glimm \& A. Jaffe, Three-Particle Structure of $\phi^{4}$ Interactions and the Scaling Limit, Phys. Rev. D 11 (1975), 2816-2827.

[34] J. Glimm \& A. Jaffe, A Tutorial Course in Constructive Field Theory, in New Developments in Quantum Field Theory and Statistical Mechanics (Cargèse, 1976), M. Levy \& P. Mitter, editors. NATO Advanced Study Institutes Series: series B: physics Vol. 26, Plenum Press, New York, 1977.

[35] — \& - Functional Integral Methods in Quantum Field Theory, in New Developments in Quantum Field Theory and Statistical Mechanics (Cargèse, 1976), M. Levy \& P. Mitter, editors. NATO Advanced Study Institutes Series: series B: physics Vol. 26, Plenum Press, New York, 1977. 
[36] — \& -, Quantum Physics: A Functional Integral Point of View, SpringerVerlag, New York, 1981 ( $2^{\text {nd }}$ edition, 1987).

[37] —, — \& T. Spencer, $P(\phi)_{2}$ Model and Other Applications of High Temperature Expansions, Part I: Physics of Quantum Field Models, in Constructive Quantum Field Theory (Erice, 1973), G. Velo \& A. Wightman, editors. Lecture Notes in Physics 25, Springer-Verlag, Berlin, Heidelberg, New York, 1973.

[38] - - — \&,$P(\phi)_{2}$ Model and Other Applications of High Temperature Expansions, Part II: The Cluster Expansion, in Constructive Quantum Field Theory (Erice, 1973), G. Velo \& A. Wightman, editors. Lecture Notes in Physics 25, Springer-Verlag, Berlin, Heidelberg, New York, 1973.

[39] - , - \& - , The Wightman Axioms and Particle Structure in the $P(\phi)_{2}$ Quantum Field Model, Ann. Math. 100 (1974), 585-632.

[40] O. W. Greenberg, Generalized Free Fields and Models of Local Field Theory, Ann. Phys. 16 (1961), 158-176.

[41] K. Gustafson \& G. Johnson, On the Absolutely Continous Subspace of a SelfAdjoint Operator, Helv. Phys. Acta 47 (1974), 163-166.

[42] R. Haag, Quantum Field Theories with Composite Particles and Asymptotic Conditions, Phys. Rev. 112 (1958), 669-673.

[43] - Observables and Fields, in Lectures on Elementary Particles and Quantum Field Theory. Brandeis University Summer Institute in Theoretical Physics 1970, Vol. 2, The MIT Press, Cambridge, Massachusetts, London, 1970.

[44] -, Local Quantum Physics, Springer-Verlag, Berlin, $2^{\text {nd }}$ edition, 1996.

[45] - \& H. Araki, Collision Cross Sections in terms of Local Observables, Comm. Math. Phys. 4 (1967), 77-91.

[46] - \& D. Kastler, An Algebraic Approach to Quantum Field Theory, J. Math. Phys. 5 (1964), 848-861.

[47] K. Hepp, On the Connection between the LSZ and Wightman Quantum Field Theory, Comm. Math. Phys. 1 (1965), 95-111.

[48] - On the Connection between the LSZ and Wightman Quantum Field Theory, in Axiomatic Field Theory, M. Chretien \& S. Deser, editors. Brandeis University Summer Institute in Theoretical Physics 1965, Vol. 1, Gordon and Breach, New York, 1966. 
[49] - Rigorous Results on the s-d Model of the Kondo Effect, Solid State Comm. 8 (1970), 2087-2090.

[50] -, R. Jost, D. Ruelle \& O. Steinmann, Necessary Conditions on Wightman Functions, Helv. Phys. Acta 34 (1961), 542-544.

[51] N. Hurt \& R. Hermann, Quantum Statistical Mechanics and Lie Group Harmonic Analysis, Part A. Lie Groups: History, Frontiers and Applications Vol. X, Math Sci Press, Brookline, s.d..

[52] D. Iagolnitzer, Analyticity Properties of the S-Matrix: Historical Survey and Recent Results in S-Matrix Theory and Axiomatic Field Theory, Acta Physica Austrica Suppl. XXIII (1981), 235-328.

[53] - Irreducibility, Analyticity, and Unitarity of Asymptotic Completeness Relations in Massive Quantum Field Theory, Fizika 17 (1985), 361-418.

[54] - Asymptotic Completeness and Multiparticle Structure in Field Theories, Comm. Math. Phys. 110 (1987), 51-74.

[55] E. Ising, Beitrag zur Theorie des Ferromagnetismus, Z. Physik 31 (1925), 253-258.

[56] A. Jaffe, Constructive Field Theory, in International Conference on Mathematical Physics (Lausanne, 1979), K. Osterwalder, editor. Lecture Notes in Physics 116, Springer-Verlag, New York, 1980.

[57] R. Jost, The General Theory of Quantized Fields, Amer. Math. Soc., Providence, Rhode Island, 1965.

[58] S. S. Khoruzhii, Introduction to Algebraic Quantum Field Theory, Kluwer Academic, Dordrecht, 1990.

[59] J. L. Lebowitz, Bounds on the Correlations and Analyticity Properties of Ferromagnetic Ising Spin Systems, Comm. Math. Phys. 28 (1972), 313-321.

[60] H. Lehmann, K. Symanzik \& W. Zimmerman, Zur Formulierung Quantisierter Feldtheorien, Nuovo Cimento 1 (1955), 205-225.

[61] - $-\&-$, On the Formulation of Quantum Field Theories II, Nuovo Cimento 6 (1957), 319-333.

[62] M. Lüscher, Construction of a Selfadjoint, Strictly Positive Transfer Matrix for Euclidean Lattice Gauge Theories, Comm. Math. Phys. 54 (1977), 283-292. 
[63] P. Malliavin et al., Integration and Probability, Springer-Verlag, New York, 1995.

[64] B. McCoy \& T. Wu, The Two-Dimensional Ising Model, Harvard University Press, Cambridge, 1973.

[65] I. Montvay \& G. Münster, Quantum Fields on a Lattice, Cambridge University Press, Cambridge, 1994.

[66] E. Nelson, Construction of Quantum Fields from Markoff Fields, J. Functional Analysis 12 (1973), 97-112.

[67] - Probability Theory and Euclidean Field Theory, in Constructive Quantum Field Theory (Erice, 1973), G. Velo \& A. Wightman, editors. Lecture Notes in Physics 25, Springer-Verlag, Berlin, Heidelberg, New York, 1973.

[68] R. Neves da Silva, Three Particle Bound States in even $\lambda P(\phi)_{2}$ Models, Helv. Phys. Acta 54 (1981), 131-190.

[69] M. O'Carroll, Analyticity Properties and a Convergent Expansion for the Inverse Correlation Length of the High-Temperature $d$-Dimensional Ising Model, Jour. Stat. Phys. 34 (1984), 597-608.

[70] — \& W. D. Barbosa, Analyticity Properties and a Convergent Expansion for the Inverse Correlation Length of the Low-Temperature $d$-Dimensional Ising Model, Jour. Stat. Phys. 34 (1984), 609-614.

[71] L. Onsager, Crystal Statistics, I. A Two-Dimensional Model with an OrderDisorder Transition, Phys. Rev. 65 (1944), 117-149.

[72] K. Osterwalder, Euclidean Green's Functions and Wightman Distributions, in Constructive Quantum Field Theory (Erice, 1973), G. Velo \& A. Wightman, editors. Lecture Notes in Physics 25, Springer-Verlag, Berlin, Heidelberg, New York, 1973.

[73] — \& R. Schrader, Axioms for Euclidean Green's Functions, Comm. Math. Phys. 31 (1973), 83-112.

[74] — \& - Axioms for Euclidean Green's Functions II, Comm. Math. Phys. 42 (1975), 281-305.

[75] E. Prugovečki, Quantum Mechanics in Hilbert Space, Academic Press, New York, 1981. 
[76] M. Reed \& B. Simon, Methods of Modern Mathematical Physics, I. Functional Analysis, Academic Press, New York, 1972.

[77] — \& - —, II. Fourier Analysis, Self-Adjointness, Academic Press, New York, 1975 .

[78] — \& -, -, III. Scattering Theory, Academic Press, New York, 1979.

[79] R. D. Richtmyer, Principles of Advanced Mathematical Physics, Vol. I, Springer-Verlag, New York, 1978.

[80] J. E. Roberts, Lectures on Algebraic Quantum Field Theory, in The Algebraic Theory of Superselection Sectors. Introduction and Recent Results, D. Kastler, editor, World Scientific, 1990.

[81] D. Robinson, Return to Equilibrium, Comm. Math. Phys. 31 (1973), 171-189.

[82] D. Ruelle, On the Asymptotic Condition in Quantum Field Theory, Helv. Phys. Acta 35 (1962), 147-163.

[83] S. Sakai, $C^{*}$-algebras and $W^{*}$-algebras, Springer-Verlag, Berlin, Heidelberg, New York, 1971.

[84] R. S. Schor, The Particle Structure of $\nu$-Dimensional Ising Models at Low Temperatures, Comm. Math. Phys. 59 (1978), 213-233.

[85] - Existence of Glueballs in Strongly Coupled Lattice Gauge Theories, Nucl. Phys. B 222 (1983), 71-82.

[86] - Glueball Spectroscopy in Strongly Coupled Lattice Gauge Theories, Comm. Math. Phys. 92 (1984), 369-395.

[87] - \& M. O'Carroll, On the Mass Spectrum of the 2+1 Gauge-Higgs Lattice Quantum Field Theory, Comm. Math. Phys. 103 (1986), 569-597.

[88] — \& - Transfer Matrix Spectrum and Bound States for Lattice Classical Ferromagnetic Spin Systems at High Temperature, Jour. Stat. Phys. 99 (2000), 1207-1223.

[89] — \& - , Decay of the Bethe-Salpeter Kernel and Bound States for Lattice Classical Ferromagnetic Spin Systems at High Temperature, Jour. Stat. Phys. 99 (2000), 1265-1279.

[90] —, J. C. A. Barata, P. A. Faria da Veiga \& E. Pereira, Spectral Properties of Weakly Coupled Landau-Ginzburg Stochastics Models, Phys. Rev. E 59 (1999), 2689-2694. 
[91] E. Seiler, Gauge Theories as a Problem of Constructive Quantum Field Theory and Statistical Mechanics, Lecture Notes in Physics 159, Springer-Verlag, Berlin, Heidelberg, New York, 1982.

[92] G. L. Sewell, Quantum Fields on Manifolds: PCT and Gravitationally Induced Thermal States, Ann. Phys. 141 (1982), 201-224.

[93] B. Simon, Correlation Inequalities and the Mass Gap in $P(\phi)_{2}$ I. Domination by the Two Point Function, Comm. Math. Phys. 31 (1973), 127-136.

[94] -, Positivity of the Hamiltonian Semigroup and the Construction of Euclidean Region Fields, Helv. Phys. Acta 46 (1974), 686-696.

[95] -, The $P(\phi)_{2}$ Euclidean (Quantum) Field Theory, Princeton Univ. Press, Princeton, New Jersey, 1974.

[96] -, Functional Integration and Quantum Physics, Academic Press, New York, 1979.

[97] — \& R. B. Griffiths, The $\left(\phi^{4}\right)_{2}$ Field Theory as a Classical Ising Model, Comm. Math. Phys. 33 (1973), 145-164.

[98] T. Spencer, The Absence of Even Bound States for $\lambda\left(\varphi^{4}\right)_{2}$, Comm. Math. Phys. 39 (1974), 77-79.

[99] - The Decay of Bethe-Salpeter Kernel in $P(\phi)_{2}$ Quantum Field Models, Comm. Math. Phys. 44 (1975), 143-164.

[100] —, The Bethe-Salpeter Kernel in $P(\phi)_{2}$, in Les Méthodes Mathématiques de la Théorie Quantique des Champs, Centre National de la Recherche Scientifique (C.N.R.S.), editor, Marseille, 1975.

[101] — \& F. Zirilli, Scattering and Bounded States in $\lambda P(\phi)_{2}$, Comm. Math. Phys. 49 (1976), 1-16.

[102] O. Steinmann, A Rigorous Formulation of LSZ Field Theory, Comm. Math. Phys. 10 (1968), 245-268.

[103] R. F. Streater \& A. S. Wightman, PCT, Spin and Statistic, and All that, Benjamin, New York, 1964.

[104] F. Strocchi, Selected Topics on the General Properties of Quantum Field Theory, World Scientific, Singapore, 1993. 
[105] C. J. Thompson, Mathematical Statistical Mechanics, Princeton University Press, New Yersey, 1972.

[106] F. J. Wegner, Duality in Generalized Ising Models and Phase Transitions without Local Order Parameters, J. Math. Phys. 12 (1971), 2259-2272.

[107] A. S. Wightman, Quantum Field Theory in terms of Expectation Values, Phys. Rev. 101 (1956), 860-866.

[108] — \& L. Gårding, Fields as Operator-Valued Distributions, Ark. Fys. 28 (1964), 129-189.

[109] K. G. Wilson, Confinement of Quarks, Phys. Rev. D 10 (1974), 2445-2459. 


\section{Índice Remissivo}

$\begin{array}{ll}A, 35 & P:=: Q:=R, \text { vii } \\ A \backslash B, \text { vii } & P=: Q, \text { vii } \\ A \triangle B, \text { vii } & P_{i}, 21 \\ A^{c}, \text { vii } & P_{x}, 21 \\ A_{1}, 35,93 & R, 36 \\ A_{2}, 35,93 & R_{00}, 44 \\ A_{3}, 94 & R_{0}, 36 \\ A_{\delta}, 48 & R_{0}^{\prime}, 58 \\ A_{\delta}^{1}, 57 & S, 56 \\ B, 35,93 & S^{\Lambda}, 16 \\ B(\mathcal{H}), \text { ix } & S_{\mathcal{A}}, \text { ix } \\ B_{1}, 35 & S_{n}, 34 \\ B_{2}, 35 & T, 35 \\ C(X), \text { viii } & T^{d}, 22 \\ C(X, Y), \text { viii } & T_{1}, 35 \\ D, 48,74 & T_{2}, 35 \\ D_{1}, 52 & T_{i}, 35 \\ D_{\text {in }}, 28 & U_{\text {in }}, 28 \\ D_{n}(\mathbb{F}), \text { ix } & V_{+}^{\text {out }}, 4 \\ E_{\left(\lambda_{0}, \lambda\right)}, 22 & X^{\prime}, \text { viii } \\ E_{\lambda}, 22 & Z_{1}, 44 \\ H_{\Lambda}, 74 & \triangle_{\delta}, 22 \\ I_{\delta}, 48 & \triangle_{i}, 22 \\ K_{1}, 58 & \Omega, 21 \\ L(\mathcal{H}), \text { ix } & \alpha f, 19 \\ M_{n}(\mathbb{F}), \text { ix } & \square, \text { vii } \\ M_{x}, 77 & \mathbb{C}, \text { vii } \\ N, 34 & \chi_{M}, 77 \\ P(A), \text { vii } & \chi_{T_{1}}, 35 \\ P F(A), \text { vii } & \chi_{T_{2}}, 35 \\ P:=Q, \text { vii } & \chi_{T}, 35 \\ P:=R=: Q, \text { vii } & \text { conn }(i, j), 77 \\ & \end{array}$




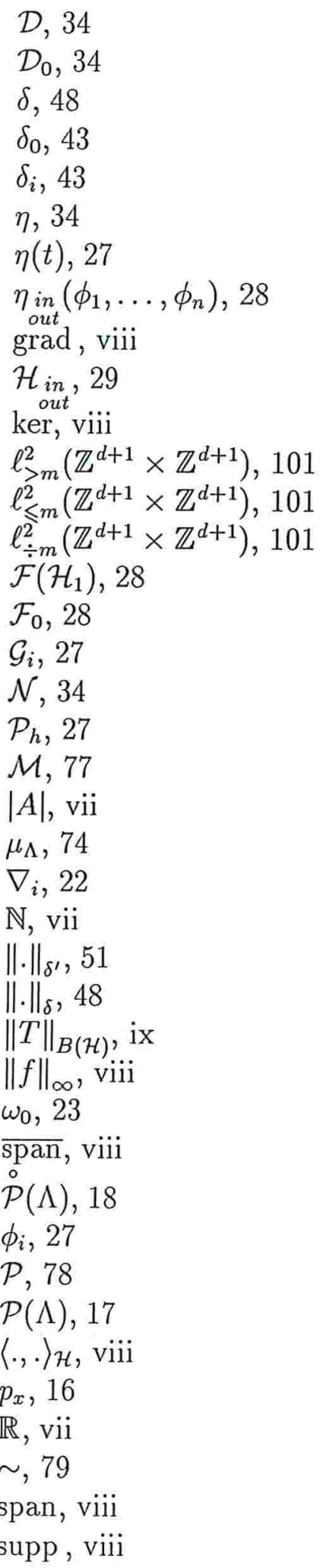

$\tau, 34$

$\tau^{\prime}, 36$

$\tau_{n}, 19$

$\tau_{x}, 19$

$\operatorname{conn}\left(i_{1}, \ldots, i_{2 n}\right), 78$

$\operatorname{diag}\left(a_{1}, \ldots, a_{n}\right)$, ix

$\theta, 19$

$\psi_{0}, 4$

$\mathcal{L}(\Lambda), 74$

$\widetilde{p}_{i}, 23$

$\xi, 34$

$\mathbb{Z}$, vii

$d \mu\left(\lambda_{0}, \lambda\right), 22$

$d \rho\left(\lambda_{0}, \mathbf{p}\right), 88$

$e_{n}, 19$

$i_{k_{m}}, 97$

$j_{L}, 74$

$j_{m}, 74$

$r_{0}(k), 58$

$u_{1}, 34$

$u_{2}, 34$

$u_{3}, 34$

$u_{4}, 34$

$\mathrm{P}, 21$

álgebra

quase-local, 6

de detectores, 13

local, 6

ímpar, vetor, 35

$\mathcal{G}^{f}, 27$

$\square$, vii

anti-simétrico, subespaço de $\ell^{2}\left(\mathbb{Z}^{d+1} \times\right.$

$$
\left.\mathbb{Z}^{d+1}\right), 94
$$

axiomas

de Osterwalder-Schrader, 5

de Gårding-Wightman, 3

de Haag-Kastler, 6

de Nelson, 7

baixo, parte de, 95 


\author{
Bethe-Salpeter \\ equação de, 34 \\ núcleo de, 34 \\ Bisognano-Wichmann, teorema de, 3
} bordas, 95

caminhos, 77

campo livre na rede, 22

cima, parte de, 95

compatíveis, polímeros, 79

completeza assintótica, 30

condição espectral, 4

cone positivo, 4

conexa, parte, 34

conexo, caminho, 77

configuração, 93

1,94

2,94

3,94

A, 95

B, 95

bordas, 95

degenerada, 93

do mesmo tipo, 93

em $\Lambda, 16$

equivalência de, 93

não-degenerada, 93

parte de fora, 95

parte de baixo, 95

parte de cima, 95

parte do meio, 95

configurações em $\Lambda, 16$

conjunto

de polos, 56

disjunto

de soluções, 27

de vectores, 27

detectores, álgebra de, 13

diferença simétrica de $A \operatorname{com} B$, vii

distribuições de Wightman, 4 equação de Bethe-Salpeter, 34

equivalentes, configurações, 93

espalhamento, teoria do, 7

na rede, 24

esquema axiomático LSZ, 5

expansão em polímeros, 80

fórmula

de Gellmann-Low, 22

de redução, 5

fatorização assintótica exponencial , 24

fora, parte de, 95

formalismo LSZ, 5

funções

de $n$ pontos, 21

de Schwinger, 5

na rede, 21

de Wightman, 4

na rede, 24

Gellmann-Low, fórmula de, 22

Gårding-Wightman, axiomas de, 3

Haag-Kastler, axiomas de, 6

Haag-Ruelle, teoria de, 8

infrapartículas, problema das, 9

infravermelho, problema do, 9

invariância, 19

Ising, modelo de, 74

Laplaciano discreto, 22

ligações, 73

limitações metodológicas, 113

locais, álgebras, 6

LSZ, esquema axiomático de, 5

matriz de transferência, 21

meio, parte do, 95

meromorfo, operador, 56

metodológicas, limitações, 113

modelo 
de Ising, 74

de Schwinger, 3

núcleo de Bethe-Salpeter, 34

números

$$
\begin{aligned}
& \text { complexos, vii } \\
& \text { inteiros, vii } \\
& \text { naturais, vii } \\
& \text { reais, vii }
\end{aligned}
$$

não-conexa, parte, 34

Nelson, axiomas de, 7

operador meromorfo, 56

Osterwalder-Schrader, axiomas de, 5

par, vetor, 35

parte

conexa, 34

de baixo, 95

de cima, 95

do meio, 95

não-conexa, 34

polímeros, 78

compatíveis, 79

expansão em, 80

polos, conjunto de, 56

positividade por reflexões, 19

problema

das infrapartículas, 9

do infravermelho, 9

projeção na coordenada $x, 16$

propriedade de cluster, 24

quase-local, álgebra, 6

rede, 16

redução, fórmulas de, 5

reflexões, 19

positividade por, 19

relação de dispersão, 26

para o campo livre, 23

sítios, 16
Schwinger

funções de, 5

na rede, 21

modelo de, 3

simétrico, subespaço de $\ell^{2}\left(\mathbb{Z}^{d+1} \times \mathbb{Z}^{d+1}\right)$,

94

suportada em, funcão ou medida, viii

Teorema

de Bisognano-Wichman, 3

de Reconstrução de Wightman, 5

Teoria

do Espalhamento, 7

na Mecânica Quântica, 31

na rede, 24

Geral de Campos Quantizados, 4

Quântica de Campos, 2

Axiomática, 3

Construtiva, 7

Euclidiana, 6

na rede, 16

tipo, configurações do mesmo, 93

TQC, 19

na rede, 16,19

transferência, matriz de, 21

translações, 19

tubo

estendido permutado, 5

positivo, 4

vácuo, 4

vetor

ímpar, 35

par, 35

vizinhos próximos, 73

Wightman

distribuições de, 4

funções de, 4

na rede, 24

Teorema de Reconstrução de, 5 


\section{Epílogo}

And I pray that I may forget

These matter that with myself I too much discuss

Too much explain

Because I do not hope to turn again

Let these words answer

For what is done, not be done again

May the judgement not be too heavy upon us

Thomas Stern Elliot Ash-Wednesday, I.

Y aquí me despido yo,

Que he relatao a mi modo

Males que conocen todos,

Pero que naides cantó.

José Hernandez

El gaucho Martín Fierro, XIII. 